Santiago Velasquez

Cost Consciousness: Conceptual Development from a Management Accounting Perspective

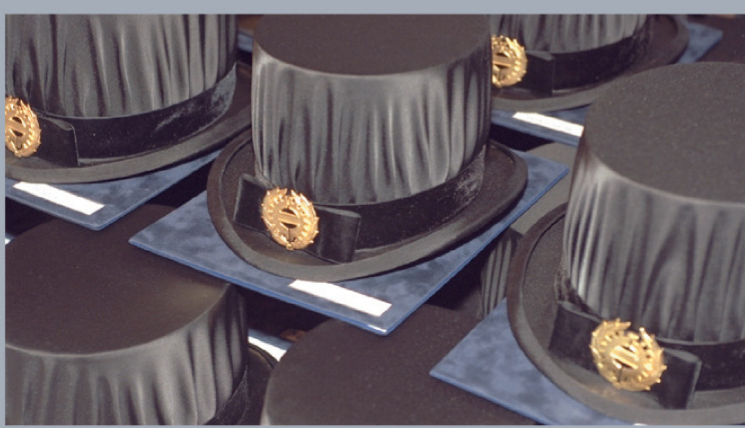

Julkaisu $1089 \cdot$ Publication 1089 
Tampereen teknillinen yliopisto. Julkaisu 1089

Tampere University of Technology. Publication 1089

Santiago Velasquez

\section{Cost Consciousness: Conceptual Development from a Management Accounting Perspective}

Thesis for the degree of Doctor of Science in Technology to be presented with due permission for public examination and criticism in Sähkötalo Building, Auditorium S4, at Tampere University of Technology, on the $9^{\text {th }}$ of November 2012, at 12 noon.

Tampereen teknillinen yliopisto - Tampere University of Technology

Tampere 2012 
ISBN 978-952-15-2939-9 (printed)

ISBN 978-952-15-3013-5 (PDF)

ISSN 1459-2045 


\section{Foreword}

Out of the basket of memories holding images that confuse reality and fantasy, one in particular I wish never to forget. At some point during the early 1980s, sitting with my father on a couch watching TV, an old cinematographic footage appeared before my eyes. I turned and asked for an explanation on why the world used to be in black and white. My father stared and corrected my infantile (and probably troubling) appreciation. This experience, however, would reflect my never-ending preference for dreams over reason, ideas over actions, pictures over words, sounds over lyrics, clouds over earth; and so, it is no surprise that the result of these past years - filled with smiles, shrugs, and sighs - dwells in the land of parallel realms where reality is constructed, society is constituted, and, of course, cost consciousness is starting to emerge.

I would like to thank my father, mother, and brother for their endless and unconditional love and support. Our family's structural pillars, following this dissertation's theoretical background, have proven to be the properties of a transoceanic and time-defiant system to which this work owes a great deal. Also in my family circle I include Tytti, my comrade in cooking and loving across the Colombian Andes, over the Iranian skies, under the Jordanian sun, and below the haunted streets of Cambridge: refer to Pulse's No. 6.

M oving to the professional arena, Prof. Petri Suomala - acting as my supervisor - is in great part responsible for guiding my interest towards this dissertation's topic and theoretical foundations which, little did I know, would provide me with a new window for a better understanding of my self. Thank you. Along these lines, I will always be grateful for the meticulous review and priceless advice that Assoc. Prof. Tomi Nokelainen has provided throughout this process. Without his contributions, the conceptualization of cost consciousness would certainly lie in a more infant stage. Furthermore, I would like to thank my pre-examiners, Prof. M arko Järvenpää (University of Jyväskylä) and Prof. Lino Cinquini (Scuola Superiore Sant'Anna), for their valuable time, comments, and insights. Also, I am grateful for the conversations with Mr. Erno Selos which have helped me clarify many of the challenging notions that roam the foreign fields of institutional theory. Moreover, I would like to express my gratitude to Mr. Oscar Franco for his kindness in allowing me to use Kopla as an illustrative case study.

I am also thankful to all the members of the Cost Management Center who have awarded me with lessons, not only on management accounting, but also on the more mundane aspects of life (e.g. mixing motorcycles and ice, reviving stubborn cars, entrepreneurship and the color black, inserting caption, karttu tossing optimization, introduction to digital albums, quick and dirty courage, wine reductions, bowties and exposure, Jämsä, and throwing logs and rocks for reasons which I still do not fully understand). In addition, I would like to thank Prof. Juho Kanniainen for molding my professional life through actions and inspiration. Furthermore, I want to extend my deep appreciation to Dr. Özgur Dedehayir for the countless coffees where my ideals on life have been demolished and rebuilt time and time again. I would also like to thank Mr. Carlos Reyes, Mr. William López, and all of my other friends and close acquaintances who have contributed in transforming my life during these last years into memories which I will always cherish. Finally, I am grateful for the support of the Department of Industrial M anagement and the funding of The Finnish Doctoral Program in Industrial Engineering and M anagement. 


\section{Abstract}

Velasquez, Santiago. 2012. Cost Consciousness: Conceptual Development from a Management Accounting Perspective.

Keywords: cost consciousness, management accounting, institutional theory, conceptualization

Cost consciousness is a notion which appears in thousands of scientific articles belonging to a wide range of disciplines. Despite its common use, the scientific community has allocated marginal attention to the understanding of cost consciousness. In this dissertation, I argue that it is important to develop this understanding for a couple of reasons. On the one hand, given that one of the objectives of scientific writing is to clearly communicate knowledge among scholars, unclear notions can be considered as unwanted elements of scientific literature. On the other hand, and certainly more important, developing our understanding of cost consciousness can potentially provide interesting and insightful new windows for research. This dissertation addresses the conceptualization of cost consciousness particularly from a management accounting perspective. In accomplishing this objective, this dissertation develops two interconnected exercises: (i) a conceptual exercise aiming to understand what cost consciousness is from the perspective of management accounting scholars; and (ii) a theoretical exercise analyzing how cost consciousness could be driven in an organization. In addition, a case company has been used to enrich the conceptualization of the notion and illustrate the way in which the formulation presented in this work could be used to analyze cases where the institutionalization of cost consciousness is of interest. With respect to the conceptual exercise, this work has rigorously identified and reviewed articles from management accounting related journals where the cost consciousness notion appears. From these, the definitions, interpretations, determinants and results which management accounting scholars have associated with cost consciousness have been identified and inferred. The results underline the difficulty in defining the notion and the perception of cost consciousness as a holistic concept that encapsulates various forms of understanding and reflecting on cost related issues. Moreover, management accounting systems have been identified as elements which may play an important role in driving cost consciousness. In addition, although the results of cost consciousness tend to be perceived as positive, the notion has also been associated with negative outcomes. With respect to the theoretical exercise, this work draws on the Burns and Scapens Framework (BSF) which is mainly based on Old Institutional Economics. Also, by considering the recent work by various scholars who have contributed and criticized the BSF, I have provided an extension of the framework and a formulation which includes and replaces various elements that appear on the original BSF. The conceptual and theoretical exercises are interconnected because the identified and inferred definitions, interpretations, determinants and results, have been brought into the extended BSF to theorize on how cost consciousness could be institutionalized from a management accounting perspective. In particularly, the results suggest that management accounting systems may play a very active role in the institutionalization of cost consciousness. M oreover, this dissertation also underlines the potential role of routines, rules, exogenous forces and agents in the institutionalization process. Therefore, the institutionalization of cost consciousness requires a mish-mash of elements and mechanisms interacting between each other. This dissertation serves as a comprehensive platform for various avenues of research on cost consciousness which could be based not any more on hunches, but on knowledge and better grounded understanding. 
I am the author of many things I do not intend to do, and may not want to bring about, but none the less do.

Anthony Giddens on agency $(1984$, p. 9) 


\section{Table of Contents}

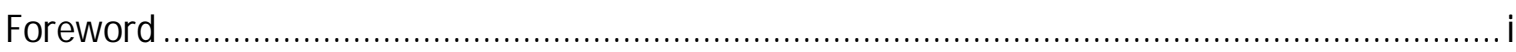

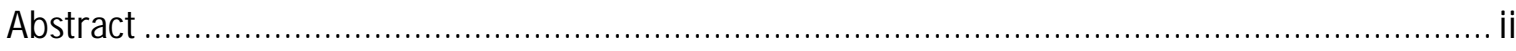

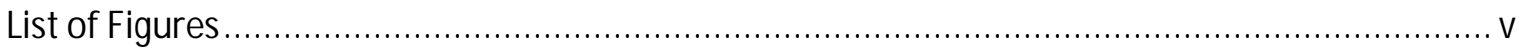

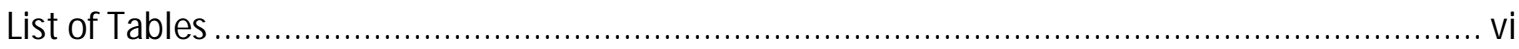

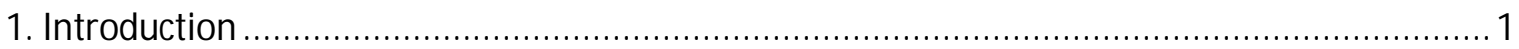

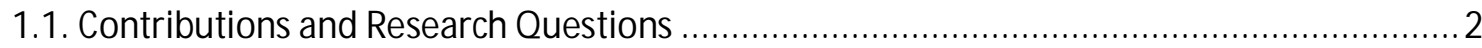

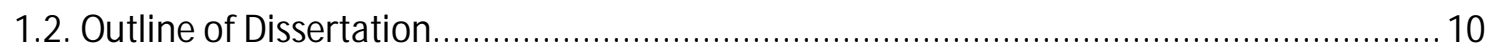

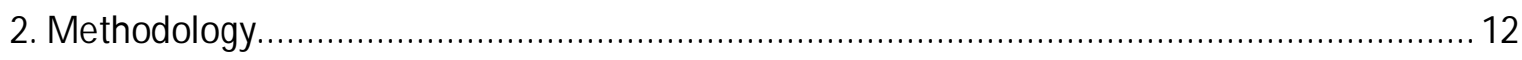

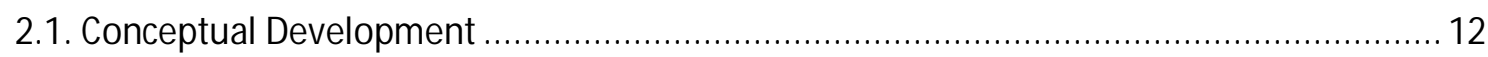

2.2. Illustrative Research Strategies........................................................................ 17

3. Cost Consciousness from a Management Accounting Perspective ..................................... 29

3.1. Linguistic Composition: Consciousness \& M anagement Accounting Practices .................. 30

3.2. Cost Consciousness: Use, Determinants, Results, and Interpretations............................ 36

3.3. Survey Research and Interviews Supporting Conceptual Exercise................................... 56

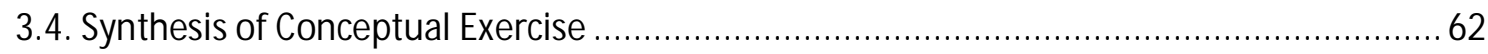

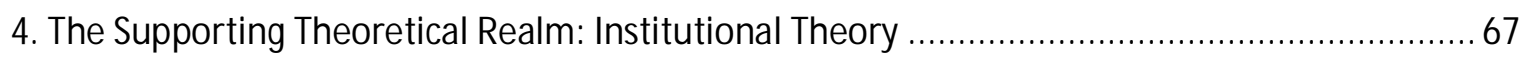

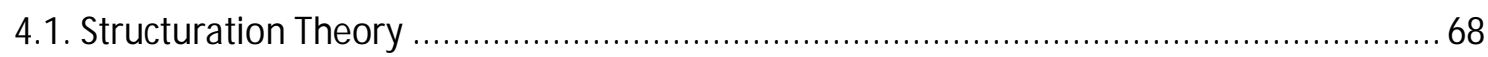

4.2. Institutional Economics: Darwin, Rules, Routines, and Institutions................................ 84

4.3. Old Institutional Economics and New Institutional Economics ...................................... 97

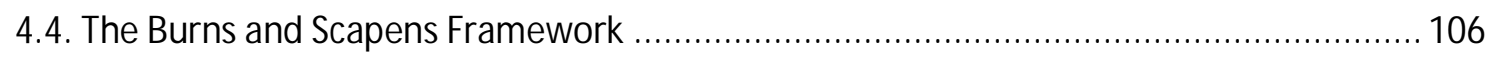

5. Management Accounting, Institutionalization, and Cost Consciousness............................ 132

5.1. Integration of Conceptual Exercise in Proposed Formulation ..................................... 132

5.2. Description of Case Study: A Colombian Food Processing Company................................ 142

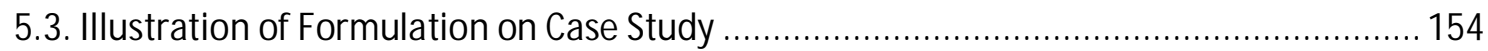

6. Discussions: Contributions, Future Research, and Limitations.......................................... 165

6.1. Contributions and Implications for Future Research .............................................. 165

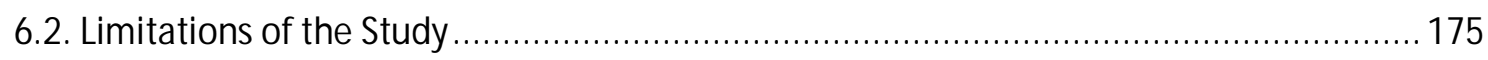

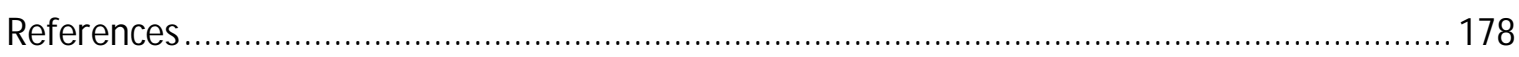

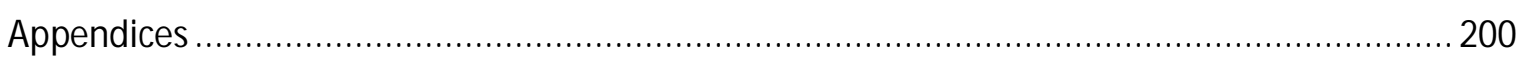




\section{List of Figures}

Figure 1. Relationship between conceptual level analysis and theorization ............................. 9

Figure 2. The scientific research cycle (Straub 1989) ........................................................ 12

Figure 3. Conceptual framework analyzing Emotional Labor (adapted from Grandey 2000)........ 15

Figure 4. High level framework for understanding a concept or phenomenon .......................... 16

Figure 5. The maturity cycle of research (Malhotra and Grover 1998) ...................................... 18

Figure 6. A view of Cost Containment in Health Care (adapted from Smith et al. 1981, p. 399) ... 47

Figure 7. Summary of determinants, interpretations and results of cost consciousness ............... 65

Figure 8. The Stratification M odel of Action (Giddens, 1984, p. 5) ......................................... 73

Figure 9. The Three Dimensions of Social Structure and Management Accounting Systems......... 83

Figure 10. Routines on ongoing systems of interaction (van der Steen 2011) ............................. 91

Figure 11. Casual view on Rules, Routines, Institutions, Routinization, and Institutionalization. .. 92

Figure 12. Relationship between cost consciousness, institutions, and organizational culture .....97

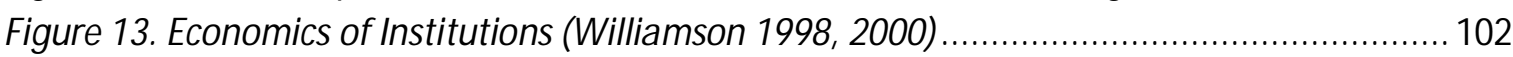

Figure 14. A sequential model of Institutionalization (Barley and Tolbert, 1997) ........................ 108

Figure 15. Interpreting management accounting change (Busco et al. 2007, p. 127) ............... 111

Figure 16. The Burns and Scapens Framework (adapted from Burns and Scapens 2000, p. 9) ... 113

Figure 17. Modified BSF for illustrating its use ................................................................ 118

Figure 18. Burns and Scapens Framework extension (adapted from Quinn 2011, p. 345) .......... 127

Figure 19. Evolutionary processes of change (adapted from Busco and Scapens 2011, p. 346) .. 130

Figure 20. M anagement Accounting Systems and aspects of routines in the BSF..................... 133

Figure 21. Formulation synthesizing relevant elements and mechanisms with respect to BSF... 134

Figure 22. Illustration of Kopla 's raw materials and final processed products.......................... 143

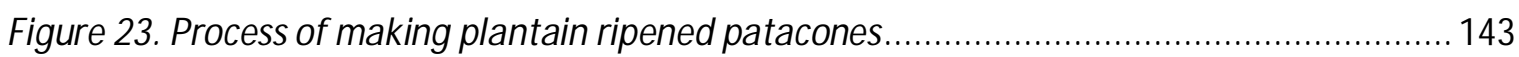

Figure 24. Kopla's organizational structure in the year 2012 .............................................. 145

Figure 25. Revenue, Gross Profit and Operating Profit of Kopla from 2007-2010 .................... 146

Figure 26. Cost structure of Kopla from 2007-2010 ............................................................. 146

Figure 27. Institutionalization of cost consciousness by powerful agent implementing a M AS... 170 


\section{List of Tables}

Table 1. Search results for "cost consciousness" or "cost conscious" in different databases......... 37

Table 2. Summary of definitions and Interpretations of cost consciousness............................... 46

Table 3. Determinants of cost consciousness from Shields \& Young and Abernethy $\&$ Vagnoni.... 50

Table 4. Summary of results associated with cost consciousness.......................................... 56

Table 5. Insights on the drivers of Cost Consciousness according to interviewees ..........................60

Table 6. Sewell's notes on depth, power, language, states, and capitalism ................................ 78

Table 7. Systems, Structures, and Agents: Definitions and Relationships................................. 79

Table 8. The dimensions of social structure (adapted from Giddens 1979, p. 82) ....................... 80

Table 9. Definitions of institutions identified .................................................................. 93

Table 10. Description of three branches of institutional theory according to Scapens (2006) ..... 105

Table 11. Various definitions of Rules, Routines, and Institutions ........................................ 114

Table 12. Company size according to Colombian Legislation and European Commission ........... 144

Table 13. Degree of Power and Status in Kopla Board members .......................................... 159 


\section{Introduction}

This dissertation aims to contribute to the field of management accounting; a statement which serves to sketch a disciplinary boundary and, furthermore, shed light on this work's first hurdle given the difficulties in reaching consensus on a denotation of the field. Singer (1961, p. 112), after noting that "If one is to discuss management accounting ${ }^{1}$ one must know what it is", introduces his own definition: "Management accounting is the activity - and its result - of collecting financial information which has any usefulness other than the satisfaction of curiosity". One could reasonably argue that if Singer's first quote would have been taken literally, research on management accounting would not have gone forward during the last fifty years given that definitions of the notion have been proposed and challenged ever since. For example, Kaplan $(1984$, p. 414) refers to the definition by the National Association of Accountants: "[management accounting is] the process of identification, measurement, accumulation, analysis, preparation, interpretation, and communication of financial information used by management to plan, evaluate, and control within an organization...". The italics were introduced by Kaplan as (what seems) a mechanism to highlight the undeserved restriction which this definition poses on the management accounting profession dismissing the use of non-financial measurements (which Kaplan encourages) such as product quality, productivity, and product innovation. Furthermore, De Loo et al. (2011) recently mentioned the current disagreement regarding the stance of the management accounting profession and the organizational roles of management accountants. In fact, Böer (2000, p. 324) states that management accountants "seem confused about who or what they are or what the field of management accounting is or should be about".

Although acknowledging the conceptual difficulties, Chenhall (2003, p. 129) defines management accounting simply as "a collection of practices such as budgeting and product costing". Furthermore, Rasid et al. (2011) argue that management accounting provides information which is essential for six organizational activities: (i) controlling the current activities of an organization; (ii) planning its future strategies, tactics, and operations; (iii) optimizing the use of its resources; (iv) measuring and evaluating performance; (v) reducing subjectivity in the decision-making process; and (vi) improving internal and external communication. Therefore, I would like to posit a definition of management accounting in an attempt to set a conceptual boundary for this dissertation: management accounting is the collection of practices aimed at identifying, measuring, accumulating, analyzing, preparing, interpreting, and communicating financial and non-financial information used by management to plan, evaluate, optimize, and control.

M oreover, management accounting scholars have allocated considerable efforts to the topic of management accounting systems (Malmi and Brown 2008, Tucker et al. 2009); perhaps as a response to the needs of both large firms and SMEs requiring adequate and sophisticated management accounting systems (MASs) to better manage their resources and enhance customer and owner/manager values (Nandan 2010, Said et al. 2011). According to Lea and M in (2000), M ASs - such as Activity Based Costing and Traditional Full Costing - can be considered as appropriate accounting principles used to provide accurate product cost information, derive the most profitable product mix decisions, minimize inventory, and maximize profit and customer service under a given manufacturing control system.

\footnotetext{
${ }^{1}$ Text in italics appears inside quotation marks in the original quote.
} 
The purposes or objectives of MASs highlight the trivial relationship between costs and the field of management accounting. Given the triviality of this relationship, I abstain myself from presenting a list of the different cost-related topics relevant to the field. Instead, I would like to turn the attention of the reader towards one cost-related topic which has received marginal attention from the management accounting community: cost consciousness.

\subsection{Contributions and Research Questions}

This dissertation is concerned with the concept of cost consciousness from a management accounting perspective. The following sections outline the two main contributions of this work which result in the formulation of this dissertation's three research questions.

\section{Outline of the First Contribution}

One of the main objectives of scientific literature is to allow members of the scientific community to communicate the work that they are doing between each other (Atkinson 2004). The communication and exposition of research, moreover, can "be interesting, erudite, or impressive, but only if it is not obscure and if it does not distort the scientific reliability of its findings" (Ogburn 1947, p. 383). Trivial solutions for improving the communication flow and exposition of research include being clear, avoiding abstract nouns, and omitting needless words (Ogburn 1947, Strunk and White 1979). Furthermore, one can reasonably argue that the use of unclear and/or abstract notions in scientific literature can result in a bestowment upon readers of a freedom of interpretation which may hinder clear communication.

Hence, one reason for furthering our understanding of an unattended construct like cost consciousness is that, by being used in its current conceptual state, it is not serving one of the main objectives of the scientific community. However, one rational option that would allow cost consciousness to serve the objective mentioned by Atkinson is, bluntly, to omit its use in scientific literature. This dissertation does not pursue this drastic option for two main reasons. First, it is simply unrealistic to expect for the members of the scientific community to suddenly concede in avoiding the use of the term given the considerable number of writers who invoke the notion. Second, even if cost consciousness is not thoroughly understood, the notion seems to provide limited trouble in communicative flow; suggesting, perhaps, the existence of shared assumptions concerning what cost consciousness could be. Therefore, for cost consciousness to serve the objectives of the scientific community, it is necessary to develop its understanding in an attempt to reduce its ambiguity. This reduction, in turn, might also provide insight into possible shared assumptions about the notion which have not yet been considered. In this way, the understanding of cost consciousness provides the possibility of conducting research oriented towards the conceptualization of a previously unattended construct.

However, although the identification and analysis of these possible interpretations is arguably interesting, this particular research task would result in a limited amount of academic opportunities which could be addressed by a handful of researchers. Therefore, one could ponder if the furthering of our understanding of cost consciousness is limited to identifying its interpretations; or, if in turn, there are additional reasons for suspecting that cost consciousness might hold more interesting and challenging research endeavors. 
Hence, in exploring the possibilities of cost consciousness to provide important and relevant opportunities for future research, I invoke Dekker (2004, p. 37):

RIB had several reasons to initiate an alliance with NMA [...] Third, an alliance was considered a good vehicle to internally manage a cultural change towards more market orientation and cost consciousness. RIB's management describes the organization as predominantly "technology driven", with less recognition of its associated costs.

From the previous quote one may reasonably infer that, according to Dekker, both market orientation and cost consciousness reflect or connote possible organizational cultures. Dekker's conception of market orientation as a business culture is shared by Narver and Slater (1990, p. 21) who define market orientation as the business culture that most effectively and efficiently creates the necessary behaviours for the creation of superior value for customers. Unfortunately, given the marginal attention that cost consciousness has received from the scientific community, verifying if the relationship between cost consciousness and organizational culture has been previously considered by other scholars would require a meticulous and time-consuming review of the literature which, as far as I am aware, is yet to be conducted. If, in fact, a relationship as such exists, considerable possibilities for research would immediately arise given the vast literature on organizational culture (Geertz 1993, Schein 2004). For instance, following the Levels of Culture by Schein (2004, p. 26), researchers could analyze possible relationships between cost consciousness and an organization's artifacts, beliefs, and basic assumptions.

Thus, the review of one quote where the cost consciousness notion is used sheds light into new and potentially fruitful possibilities of research concerning the notion. Therefore, one could reasonably argue that, in certain cases, research opportunities only become evident if some degree of research is allowed.

Moreover, Dekker's convenient choice of words - allowing the comparison between market orientation and cost consciousness - can be used to clearly outline this work's first contribution. From a linguistic point of view, both concepts are endocentric compounds joining a noun (i.e. market and cost) acting as a modifier, with another noun (i.e. orientation and consciousness) acting linguistically as a head. In addition, from a conceptual point of view, one can reasonably argue that the heads of these two concepts are arguably more challenging when compared to their respective modifiers. Hence, as the head is the part containing the basic meaning of the whole compound (the task of modifiers is to restrict meaning), an abstract head drives ambiguity into the compound which it composes ${ }^{2}$. Therefore, the abstract natures of cost consciousness and market orientation find a common linguistic explanation. However, the state of conceptual development of these two notions is, for now, very different. While cost consciousness remains vague and quite unexplored, market orientation has received considerable attention since the 1950s (Piercy et al. 2002) and, furthermore, has witnessed an important increase of academic and practitioner interest since the 1980s (Harris and Ogbonna 2001). Actually, the considerable attention on furthering the understating of market orientation has led some scholars to describe the notion as a corporate state of mind or a philosophy of business management (Kirca 2010).

The vast difference between market orientation and cost consciousness, in terms of conceptual development, may be sensed by conducting a quick search using certain scientific databases. Searching for peered review journals which include market orientation in their title can result in

\footnotetext{
${ }^{2}$ Similar examples are found throughout the management accounting literature including results orientation, financial awareness, and product orientation.
} 
hundreds of hits, contrasting, quite sharply, the handful of journals which include cost consciousness in their title ${ }^{3}$. Furthermore, van Raaij and Stoelhorst (2008), through a literature review on market orientation, collected six main definitions of market orientation, two main scales for measuring market orientation, sixteen different references discussing the consequences of market orientation, fifteen articles studying the variables which serve as potential moderators in market orientation, thirteen works analyzing the antecedents or drivers of market orientation, nine major approaches for implementing market orientation, and twelve articles analyzing the managerial implications of its implementation. Therefore, although cost consciousness and market orientation share a similar abstract nature; while the former remains conceptually vague, the latter has received considerable attention resulting in its robust conceptual development.

Hence, it would be interesting to identify the characteristics which have allowed market orientation to receive such attention in order to assess if cost consciousness has what it takes to follow a similar path. As far as I am concerned, this particular topic has not been addressed; therefore, I will briefly discuss two reasonable hypotheses. On the one side, the interest on market orientation could derive from a mere chain of coincidences (e.g. a moment of inspiration by a particular individual followed by a group of bright and influential scholars who decide to take note of the notion and pursue its conceptual development). Although a coincidence as such is certainly plausible, from my perspective, an important driver of market orientation's success has been, paradoxically, its inherent abstract nature which results from its particular linguistic composition. While the abstract head of market orientation (i.e. orientation) drives abstractness into the meaning of the compound, its modifier (i.e. market) narrows down the conceptual realm of the compound by restricting orientation into meanings that involve, for example, marketing and market segments. Therefore, although market orientation serves as a tool to cover a very broad range of issues (thanks to its abstract head), it does so in a restricted conceptual realm (thanks to its restricting modifier).

I believe that the linguistic composition shared by market orientation and cost consciousness has had a considerable role in supporting the exploitation of the former by the academic community. For example, the notion's composition has allowed Narver and Slater (1990) to argue that market orientation consists of customer orientation, competitor orientation, and interfunctional coordination. Each one of these three notions share similar linguistic compositions when compared to market orientation (or cost consciousness). However, as one can reasonably argue that a market is composed of customers and competitors, the conceptual realm of customer and competitor orientation is narrower when compared to market orientation (e.g. a market is composed of customers and competitors), allowing Narver and Slater to easily include them inside the latter's conceptual realm. Hence, a sort of conceptual snowball effect is created as efforts related to customer orientation may simultaneously develop market orientation.

In an analogous manner with respect to market orientation's linguistic head, consciousness is considered to be puzzling (Flanagan 1992, p. xi), controversial (Kauffman 2008, p. 197) and a hard task for anyone attempting to explain what it is (Dennett 2001). However, cost consciousness's modifier (i.e. cost) narrows down the conceptual realm of the compound. In this way, although one could imagine the possibility of cost consciousness serving as a topic of

\footnotetext{
${ }^{3}$ On January $31^{\text {st }} 2012$ ProQuest identified 695 peered reviewed articles containing market orientation in their title (EBSCO identified 655, ScienceDirect identified 140, Emerald identified 204, and JSTOR identified 14). On the same day ProQuest identified 5 articles containing cost consciousness in their title (EBSCO: 10; ScienceDirect: 3; Emerald: 0; and JSTOR: 1)
} 
interest in political science and history related studies, given its linguistic composition it would most likely find a broader adoption in a field (or fields) where costs are of particular interest (e.g. management accounting, healthcare). Therefore, this dissertation serves as a call for management accounting scholars to take note of the possibilities for research which may be residing in the unattended cost consciousness notion.

However, searching for cost consciousness as a search-phrase ${ }^{4}$ in a major scientific journal database - such as EBSCO, Emerald, Science Direct, or JSTOR - identifies thousands of articles belonging to different disciplines including accounting, economics, health care, computer sciences, technology management, marketing and law. However, whenever authors use the construct in describing of a particular object - such as a customer or an organization - they rarely place any effort on clarifying their interpretation of what cost consciousness is or could be. Actually, to my best knowledge, there are only two articles from management accounting journals that provide an explicit definition of cost consciousness (Kurunmäki 1999, Nor-Aziah and Scapens 2007). These definitions (i.e. "economic judgment of operation" and "economic efficiency" respectively) are, however, quite abstract and provide a limited understanding of the authors' interpretations. Moreover, there is less than a handful of management accounting articles which have evaluated (to some extent) the cost consciousness notion. Shields and Young (1994), for example, analyze sixteen possible determinants (i.e. drivers) of cost consciousness. Their results suggest that while cost management knowledge and cost budget participation are directly related to cost consciousness, an increase in the cost of regulation decreases cost consciousness. Furthermore, the study by Abernethy and Vagnoni (2004) - which uses the instrument developed by Shields and Young (1994) for evaluating possible drivers of cost consciousness - suggests that (i) accounting information systems increase cost consciousness, (ii) there is no direct relation between formal authority and cost consciousness, and (iii) there is an indirect relationship between informal authority and cost consciousness. Although the results of both studies are comparable (given that they use the same instrument), the seven questions used to evaluate cost consciousness are based on no existing conceptual development of the notion. Therefore, despite the comparability of these studies, the central notion remains as a fairly vague, abstract, and poorly understood concept in the field of management accounting.

Thus, I would like to raise three main points from the discussions in this section. First, cost consciousness's linguistic composition should be considered as a strength (rather than a weakness) with respect to potential conceptual development endeavors that could be embraced by numerous researchers in the future. Second, given the present state of cost consciousness's conceptual development, cost consciousness's potential is yet to be uncovered. Third, given cost consciousness's current state of conceptual development, one reasonable option for uncovering its potential is to conduct a thorough and comprehensive research on how the notion has been interpreted by management accounting scholars. Hence, understanding cost consciousness is important not only because it's current state does not serve the objectives of the scientific community, but because the concept's inherent characteristics may allow a considerable amount of research opportunities which can only be uncovered by developing its conceptual base.

Moreover, cost consciousness's conceptual development may be approached in various ways. From the literature review of market orientation by van Raaij and Stoelhorst (2008) some options may be inferred: defining cost consciousness, measuring cost consciousness, identifying the causes and effects of cost consciousness, and discussing the managerial actions to implement cost consciousness. Defining cost consciousness might be a very interesting conceptual

\footnotetext{
${ }^{4}$ Some sources may refer to search-phrases as keywords
} 
endeavor. However, without any support, defining the notion would most likely prove to be a very challenging task (if not impossible) given cost consciousness's linguistic composition. Also, a lack of definition or understanding of the concept's interpretations would also make measuring cost consciousness a rather difficult endeavor (i.e. how can you measure something that you do not understand?). Furthermore, studying how managers could implement cost consciousness also requires achieving a minimum level of conceptual development.

From the list of options above, the identification of the causes and effects of cost consciousness may serve as a proper starting point for a couple of reasons. First, it allows waving off the challenges represented by defining the construct. Second, it can reasonably be approached by conducting a literature review identifying the antecedents and results which management accounting scholars have attributed to cost consciousness. Webster and Watson (2002) state that an effective literature review is not only an essential feature of any academic project, but it also creates a firm foundation for advancing knowledge, facilitates theory development, closes areas where a plethora of research exists, and uncovers areas where research is needed. Third, this literature review may, simultaneously, shed light in the existing interpretations and definitions of cost consciousness. Fourth, understanding the antecedents and results could, in the future, allow the construction of measurement scales, and analyses about the managerial requirements to implement cost consciousness. Hence, the initial literature review should focus on identifying the drivers and results (together with the definitions and interpretations) that management accounting scholars have associated with cost consciousness.

Therefore, the first contribution of this dissertation is to conduct a thorough literature review identifying the drivers, definitions, interpretations, and results which management accounting scholars have associated to the notion of cost consciousness. This first contribution is expected to shed light on possible future research endeavors and, in addition, build ground for saving the notion from remaining simply as a convenient word for muddying the scientific prose.

Finally, this first contribution culminates in the first research question which this dissertation aims to answer:

Research Question 1: What is cost consciousness as it is understood in the management accounting scientific literature?

Following the discussion in this section, this first research question would be supported by the following sub-questions: what are the drivers, definitions, and results of cost consciousness?, have management accounting scholars made associations between cost consciousness and organizational culture?, and what interesting research endeavors are raised from the analysis of these associations?

\section{Outline of the Second Contribution}

In the previous section I highlighted different ways to approach the conceptual development of cost consciousness from a management accounting perspective. One reasonable option is to identify the drivers, interpretations, and results (i.e. the first contribution of this dissertation) which management accounting scholars have associated to cost consciousness. Although the main purpose of the first contribution is to develop the foundations for the conceptualization of cost consciousness and, simultaneously, uncover potential research opportunities, by conducting 
a study as such it may be possible to take stock on how cost consciousness may become an organizational institution.

In an attempt clarify, I invoke Huntington's (1968, p. 12) definition of institution which is a "stable, valued, recurring pattern of behavior". M oreover, while Burns and Scapens (2000) argue that an institution represents the unquestionable way of doing things, Scapens (2006) states that institutions are the taken for granted ways of thinking in an organization. Therefore, one can reasonably argue that cost consciousness could be considered an organizational institution if it would reflect a particular set of stable, valued, recurring, unquestionable, and taken for granted ways of thinking in an organization ${ }^{5}$.

Although a thorough study on the public interest in understanding how to institutionalize cost consciousness is yet to be conducted (to my acknowledgement), some scholars seem to suggest that cost consciousness is in fact a welcomed phenomenon:

Cost consciousness emerged in the 1970s to become the major issue of the 1980s. We learned that there were new ways to become efficient and more productive. (Jenks 1987, p. 2)

Cost-consciousness at every step is critical to ensure optimum mechanical and commercial performance of a facility that can have a lifespan of half a century or more. (Jenkins et al. 2005, p. 1)

During this era of uncertainty in health care, cost-conscious tactics are being implemented widely among U.S. hospitals. Diseases that consume billions of health care dollars, such as CHF [Congestive Heart Failure], have come to the forefront for consideration. (Paul 1997, p. 486)

As the reader may note, the previous references suggest that by increasing cost consciousness, an organization may benefit from more efficiency, better commercial performance, and responsible cost reduction. Moreover, other scholars have noted unwanted repercussions which may arise from cost consciousness. Chenhall (2008, p. 529), for example, states: "Cost consciousness is seen to lead to distraction and a myopic vision of the organization". Thus, scientific literature suggests the existence of a latent interest in understanding how to institutionalize cost consciousness in order to access its benefits or avoid its repercussions.

However, despite the latent interest on understanding the institutionalization of cost consciousness, not only are there just a couple of management accounting studies identifying and evaluating drivers associated with cost consciousness; but, furthermore, there are no studies (to my knowledge) aiming to analyze the elements which may be involved in the institutionalization of cost consciousness. Thus, a study which furthers our understanding on the elements which may be involved in this process is yet to be developed.

Therefore, the second contribution of this dissertation is the conduction of a theoretical exercise aiming to identify and analyze the organizational elements and mechanisms which may contribute to the institutionalization of cost consciousness from a management accounting perspective. Furthermore, a perspective as such can be supported in at least three ways. One

\footnotetext{
${ }^{5}$ Furthermore, a relationship between cost consciousness as an institution and organizational culture surges once again as the latter is defined as a pattern of shared basic assumptions (Schein 2004) incorporating the habits, attitudes, beliefs, and values which permeate the individuals comprising an organization (Hall 1993).
} 
way is to conduct the theoretical exercise by drawing on theories and theoretical frameworks which have supported management accounting studies and, furthermore, have contributed considerably in the field. A second approach is to integrate the results from the first contribution (i.e. drivers, interpretations, and consequences associated to cost consciousness by management accounting scholars) in the theoretical exercise by analyzing the possible relationships between those results and the elements which may be involved in the institutionalization of cost consciousness. This integration, moreover, not only supports the management accounting perspective, but also furthers the conceptual development of cost consciousness. A third way is to illustrate the theoretical exercise with the use of a case study describing the institutionalization of cost consciousness through management accounting means.

Finally, the second contribution culminates into the second and third research questions of this dissertation:

Research Question 2: Which organizational elements and mechanism can contribute to the institutionalization of cost consciousness?

Research Question 3: How do the organizational elements and mechanisms contributing to the institutionalization of cost consciousness interact between each other?

Following the discussions in this section, Research Question 2 is supported by answering the subquestion: what Institutional framework can support the theorization of the institutionalization of cost consciousness? Moreover, Research Question 3 would be supported by answering the subquestions: what role, if any, do the drivers, interpretations, and results associated with cost consciousness may play in the institutionalization of cost consciousness? And how can the results of a case study illustrate the applicability of the theorization exercise? 
Drivers, Interpretations,

Definitions and Results of cost consciousness

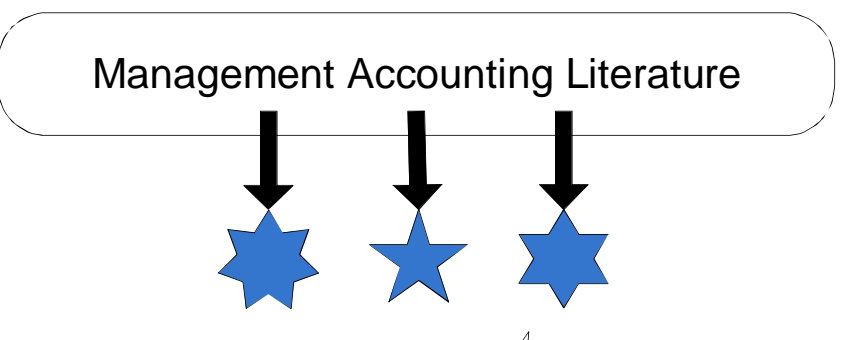

How elements interact in

institutionalization of cost

consciousness

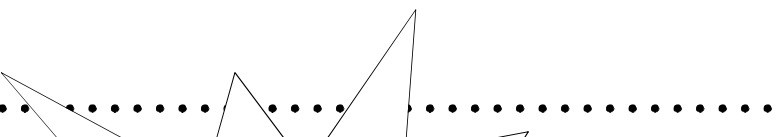

Elements that may

2

be involved in

institutionalization of cost consciousness

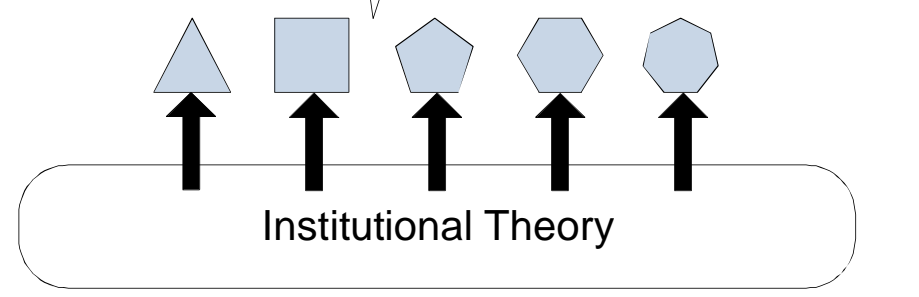

= Research Question

Figure 1. Relationship between conceptual level analysis and theorization

Figure 1 should serve as summary and graphical representation of the relationship between the three research questions of this dissertation. As it can be observed, answering the Research Question 3 requires the identification of the drivers, definitions and results which management accounting scholars have associated with cost consciousness (Research Question 1); and, furthermore, the identification of the elements which could be involved in the institutionalization process (Research Question 2).

\section{M ore on Research Questions and Objectives}

According to Yin (1994, p. 7) defining the research questions is probably the most important step in any research study. The key, Yin argues, is to understand that research questions have both substance (i.e. 'what' is my study about?) and form (i.e. am I asking a 'who', 'what', 'where', 'why', or 'how' question?). With respect to substance, one can reasonably infer what this dissertation is about as each research question derives from this work's two contributions. Moreover, the form of the research questions can be inferred from the sub-questions which clearly ask 'what' (i.e. what Institutional framework can support the theorization of the institutionalization of cost consciousness?), 'how' (i.e. how can the results of a case study illustrate the applicability of the theorization exercise?), and 'why' (i.e. why is cost consciousness desired/undesired?) inquiries. 
Moreover, Research Question 1, answered through a deductive theoretical methodology, serves the main goals of any review work, namely to bring together different views on a particular topic in order to take stock of the these views, structure them, and build the foundations not only for this study but for any future research interested in cost consciousness. For practical purposes, the process of answering Research Question 1 will also be referred to as the conceptual exercise.

Furthermore, Research Question 2 and Research Question 3 will be answered through a theoretical exercise using an established framework that supports the analysis of the elements and mechanisms which interact in institutionalizing cost consciousness from a management accounting perspective. For practical purposes, the process of answering Research Question 2 and Research Question 3 will be referred to as the theoretical exercise.

Finally, in an attempt to clarify the steps for answering the research questions, the following activities will be followed:

I. Conducting a literature review on cost consciousness to identify and analyze the drivers, results, and, if possible, interpretations and definitions, which management accounting scholars have attributed to the notion.

II. Conducting a literature review on a theoretical realm which could support the theorization on the institutionalization of cost consciousness.

III. Identifying the elements and mechanisms which may be involved in the institutionalization of cost consciousness.

IV. Analyzing possible associations between the results on Activity I and the elements and mechanisms identified in Activity III.

V. Providing an illustration which supports the theorization on the institutionalization of cost consciousness.

Clearly, while Activity I is directed towards answering Research Question 1, the four remaining activities aim at answering Research Question 2 and Research Question 3. However, the results of Activity IV also contribute to Research Question 1 as they allow further understanding of the cost consciousness notion from a management accounting perspective and can shed light on the research opportunities related to the concept. Hence, the notions of conceptual exercise and theoretical exercise do not establish solid boundaries; in other words, aspects of the former are developed in the latter (and vice versa).

\subsection{Outline of Dissertation}

This dissertation is composed of six main chapters. M oreover, these chapters are composed of various subchapters which are divided between sections and, in a couple of cases, subsections. While the title of each section appears in bold letters, the title of each subsection appears in italics. The following structure describes the main chapters and subchapters of this dissertation: 
Chapter 1 Introduction:

Discussion on the main contributions

Presentation of research questions

Outline of Dissertation

Chapter 2 Methodology:

Discussion on (selected) Research Strategies

Brief introduction into the empirically-oriented Research Strategies

Discussion on Validity of Results

Chapter 3 Cost Consciousness from a Management Accounting Perspective:

Linguistic Composition of the Notion

Identification of Drivers, Interpretation, and Results of Cost Consciousness

Analysis of the results of the survey and set of interviews

Synthesis of Conceptual Exercise

Chapter 4 Theoretical Realm Supporting the Dissertation's Contributions:

Literature Review on structuration theory

Discussion on suitability of Institutional Economic branches for supporting this study

The Burns and Scapens Framework and Extensions

Chapter 5 Institutionalization of Cost Consciousness:

Integration of Conceptual Exercise and Literature Review on institutional theory

Illustration of Applicability of Theorization with Case Study

Synthesis of Theorization Exercise

Chapter 6 Discussion on Conclusions, Future Research, and limitations

Discussion on Conclusions and Future Research

Limitations of the Study

Following the discussions on this introductory chapter, the structure above already presents further insight into the discussion that will follow. I would like to point out a couple of issues. : Firstly, the results of a survey and a set of interviews will provide further support for the conceptual exercise held in Chapter 3. Furthermore, a review on structuration theory will accompany the literature review on institutional theory in Chapter 4 as it allows clarification of certain constructs which, I considered, deserved further insight after reviewing institutional theory. Finally, the Burns and Scapens Framework (BSF) has been selected as an appropriate framework to conduct the theorization exercise. 


\section{Methodology}

This chapter discusses the research strategies employed in answering the research questions and, furthermore, in following the activities presented in the previous chapter.

As this dissertation is centered on a largely unattended and unconsidered concept - cost consciousness - its main role is to act as an exploratory study offering conceptual and theoretical foundations paving way for future research which can opt for a continuation of conceptual development and/or towards more empirical oriented strategies. In this sense, this dissertation focuses on the exploratory and conceptual refinement side of the research cycle (Figure 2).

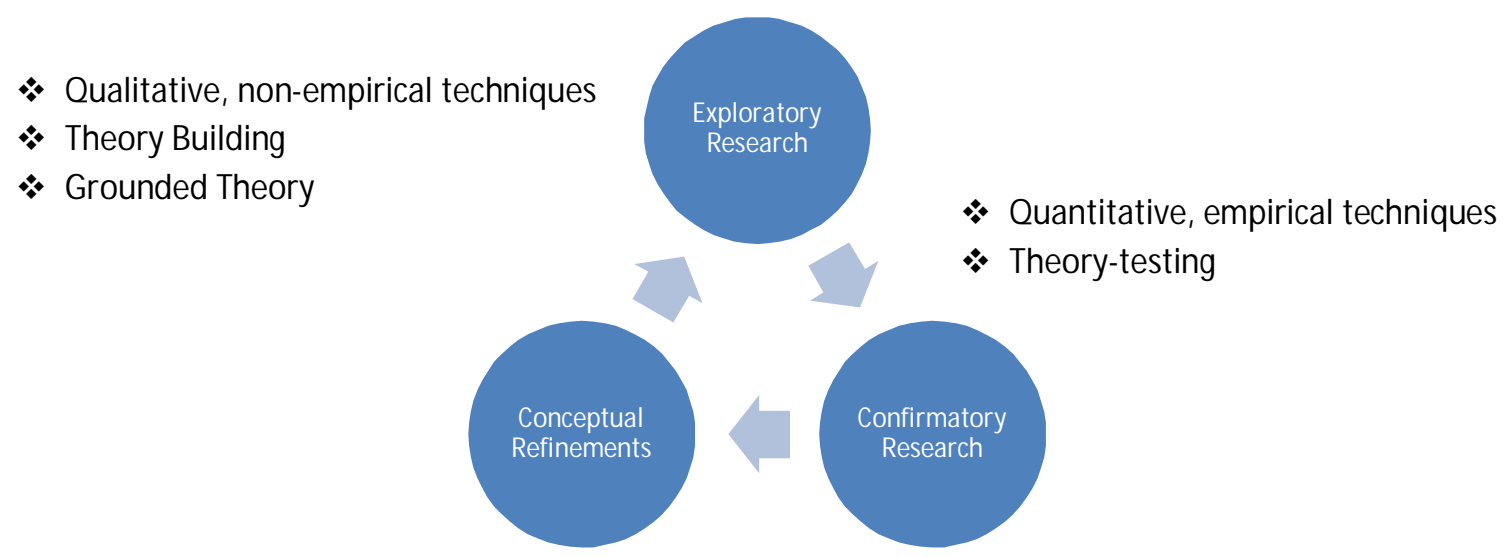

Figure 2. The scientific research cycle (Straub 1989)

As the reader may observe, the cycle indicates that for exploratory research and conceptual refinements, theory building accompanied by qualitative and non-empirical techniques is suggested. Qualitative research is the kind of research that produces findings arrived from realworld settings where the phenomenon of interest unfolds naturally seeking illumination, understanding and extrapolation to similar situations (Golafshani 2003). In qualitative research, interviews and observations are dominant in the interpretive paradigm, Golafshani adds. This dissertation uses a set of interviews and an illustrative case study to support the findings of the conceptual and theoretical exercises. In addition, the results of a survey provide practical motivation for the understanding of cost consciousness.

\subsection{Conceptual Development}

This dissertation is in great part a conceptualization exercise focusing on the notion of cost consciousness from a management accounting perspective. Therefore, a discussion on concepts is relevant. Murphy (2002) states that the psychology of concepts is like other areas of psychology where a phenomenologically simple cognitive process (e.g. walking) turns out to be a deep and maddeningly complex problem. Also hinting to the difficulties related to concept, Margolis and Laurence (1999, p. 305) quote Ray Jackendoff: "asking a psychologist, a philosopher, or a linguist what a concept is is much like asking a physicist what mass is [...] the term plays a certain role in a larger world view that includes the nature of language of meaning an, and of mind".

Arguably, one of the main difficulties related to the notion of concepts is related to the lack of consensus on the definition of the notion of concept (Nokelainen, 2008). Moreover, according to 
Margolis and Laurence (1999) there is not even an agreement with respect to what the object of the definition is (e.g. objects or cognitive/behavioral abilities of some sort). However, Dortmans et al. (2006, p. 885) have defined concepts as "an aggregation of ideas or thoughts on how to achieve some observable effect or to meet some strategic requirement". Hence, despite a lack of consensus on the denotation of the notion of concept, some authors have indeed attempted to provide very broad denotations.

Furthermore, Cerulo (2000) argues that concepts are the seeds of social research as they stand at the very heart of knowledge production. Moreover, Margolis and Laurence (1999) consider concepts as "the most fundamental constructs in theories of the mind" and argue that there is "no reason why concepts can't be mental representations". Murphy (2002) states that concepts are "a kind of mental glue that holds our mental world together." Therefore, it also seems that various authors consider the understanding of concepts as an important task regardless of the lack of consensus related to its possible denotations.

In fact, the understanding of concepts has been approached through theories of concepts. In this chapter I will discuss the following: the classical view, the prototype view, and the exemplar view. The classical view, also known as the definitional view, argues that most concepts encode the conditions that are necessary and jointly sufficient for something to be in its extension (M argolis and Laurence 1999). According to Belohlavek et al. (2009), the classical theory holds that the meanings of words and their associated concepts can be completely defined and are contained in clear boundaries. M urphy (2002) elaborates by explaining the three main claims of the classical view: (i) concepts are mentally represented definitions which provide characteristics that are necessary and jointly sufficient for membership in the category ${ }^{6}$; (ii) every object is either in or not in the category, and (iii) there is no distinction between category members. A widely used example to illustrate the classical view is BACHELOR (Margolis and Laurence 1999, Nokelainen 2008). The concept BACHELOR implicates the concepts MAN, ADULT, and UNM ARRIED which are necessary and jointly sufficient. In this example, the boundaries are sharp and fixed: a man who is adult and unmarried is a bachelor.

The classical view, however, was led to its full rejection in the 1970s, partly because of the work by Eleanor Rosch and by the convincing experimental evidence that conceptual categories never have sharp boundaries (Belohlavek et al. 2009, Murphy 2002). Furthermore, Margolis and Laurence (1999, p.27) explain that there are few, if any, examples of unambiguously defined concepts; and, furthermore, that it is possible to have a concept in spite of massive ignorance and/or error, indicating that concept possession cannot be a matter of knowing a definition. In the case of COST CONSCIOUSNESS (from a management accounting perspective) the definitional structure could reasonably be considered to be complex but could include, for example, ATTENTIVE TO BUDGETS and KNOWLEDGEABLE OF COSTING THEORIES.

The rejection of the classical view allowed a number of early alternatives which are attributed to a single theory, the prototype view. The name of this view derives from the idea that a single prototype could represent a whole category (Murphy 2002). Furthermore, the prototype view is considered as a summary representation where members of a particular category are graded in order to assess their degree of membership in the category (Belohlavek et al. 2009). Considering the concept BIRD may clarify the prototype view. BIRD is composed of features such as ANIMAL, FLIES, SINGS, NESTS, and LAY EGGS. Hence, BIRD represents the prototype and the main

\footnotetext{
${ }^{6}$ M urphy $(2002$, p. 5) uses "the word concepts to talk about mental representation of classes of things, and categories to talk about the classes themselves".
} 
category. Moreover, under the prototype view, a PARROT is a clear member of BIRD because it tends to have all of the previous properties. Moreover, although PENGUIN connotes an animal that does not fly, it does have enough of the properties assigned to BIRD for the concept BIRD to apply to PINGUIN. In other words, grading the concept of PINGUIN with respect to the BIRD prototype qualifies PINGUIN as a member of BIRD. Furthermore, the concept of BAT is composed of ANIM AL and FLIES. However, it is not composed of the other features which prove to be more important for establishing the BIRD concept. Therefore, grading the concept of BAT with respect to BIRD, does not qualify BAT as a member of BIRD. Moreover, it is useful to note that PINGUIN could not be conceptualized as a BIRD from the classical view perspective. Hence, this example highlights an advantage of the prototype view with respect to the classical view. However, consider the concepts SECOND-DEGREE COUSIN OF HUGO CHAVEZ, or NOT A WOLF. As people, in certain cases such as with these concepts, simply do not have views about the central tendencies of the corresponding categories, many concepts lack prototypes.

Furthermore, if the features of COST CONSCIOUSNESS would be, for example, ATTENTIVE TO BUDGETS, KNOWLEDGEABLE OF COSTING THEORIES, and PERIODICAL INVESTMENT IN ERP SYSTEMS, an organization would not need to have all of these features, but enough of them to be considered to be cost conscious. The problem arises, then, in assessing what enough could represent.

The exemplar view is relatively new and has not been extensively developed (Margolis and Laurence 1999). The exemplar view dismisses the idea that people have a representation that somehow encompasses an entire concept (Murphy 2002). Furthermore, this view holds that concepts are represented at least in part by their exemplars rather than by an abstract summary (M argolis and Laurence 1999). In other words, the exemplar view suggests that a concept may be defined only through the properties of those empirical entities that are already identified and known to be instances of the concept (Belohlavek et al. 2009, Nokelainen 2008). Hence, in many respects the exemplar view is radically different from the classical and the prototype views. For example, the concept of DOG of any particular person might be a set of a given number of dogs which a person remembers. If a person sees something moving in the street, and after consulting his/her memory the thing that he/she sees walking in the street refers to an exemplar that belongs to DOG, then he/she will conclude that it is a dog. The moving thing could have been slightly similar to a GOAT and perhaps mildly similar to a DONKEY, but given that it was mostly similar to DOG, then there is more evidence that the walking object is a dog.

Furthermore, the exemplar view holds that a definition of cost consciousness is not necessary as one could assess if a particular organization is cost conscious according to previous organizations which one has considered to be so.

In addition, there are other views on concepts which this subsection does not review. For example, the knowledge view, also known as the theory view of concepts, argues that "concepts are part of our general knowledge about the world" (M urphy 2002, p.60) and "people use their prior knowledge to reason about an example in order to decide what category it is, or in order to learn a new category" (Murphy 2002, p. 61). However, Murphy warns that as the knowledge approach arose as a reaction of the prototype and the exemplar views, an understanding of the knowledge view requires a thorough scrutiny of these other two views which, one can reasonably argue, is out of the scope of this work.

Furthermore, Nokelainen (2008) states that while the prototype and the exemplar views may better suit concrete concepts such as APPLE, PENCIL, or ZEBRA; the classical view may provide 
better support for the conceptualization of abstract concepts like COMPETITIVE ACTION, or, in the case of this dissertation, COST CONSCIOUSNESS. Hence, I will adopt the classical view concerning the conceptual exercise in this dissertation.

\section{How to Understand Cost Consciousness}

According to Jones and Coviello (2005) "one of the problems in conceptualizing any complex phenomenon is in trying to find a balance between very precise and casual models that tend to be narrow in their focus, and broader universal models that offer general description but are challenging to operationalise". Moreover, as Walsh (1995) states, "it is difficult for anyone to take stock of what is known and unknown about a topic when the research has not been productively synthesized". Following these statements, and in an attempt to contribute to the understanding of cost consciousness, I first place my concerns on identifying a solution for organizing the information related to the notion in a synthesized and structured manner. However, this concern brings the following question forward: what aspects of cost consciousness should be identified? For this, I turn towards works focused on conceptualizing troublesome notions. One of these, in the field of psychology, is Grandey (2000) who examines the different approaches in conceptualizing emotional labor. The author explores three main perspectives in an attempt to develop the conceptual exercise. The first perspective, derived from Hochschild (1983), considers emotional labor to be "the management of feeling to create a publicly observable facial or bodily display" and suggests that by managing emotions, employees achieve organizational goals. The second perspective, referring to the work by Ashforth and Humphrey (1993), defined emotional labor as the act of displaying appropriate emotions with the goal to engage in a form of impression management for the organization. The third perspective is M orris and Feldman's (1996) who defined emotional labor as "the effort, planning, and control needed to express organizationally desired emotion during interpersonal transactions". From the analysis of these perspectives, Grandey (2000) proposes a conceptual model of emotional labor with three main sections: situational antecedents which act as a cue from which emotions result, the emotional regulation process, and the consequences related to emotional labor (Figure 3).

Antecedents: Situational Variables

Emotion Regulation Process

Long-tern Consequences

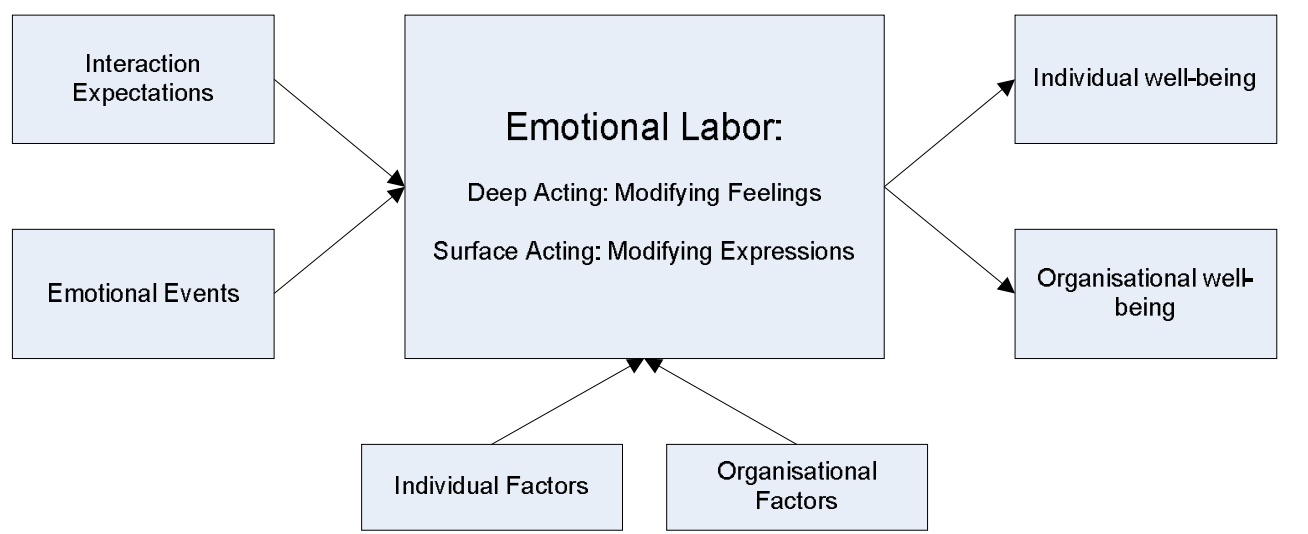

Figure 3. Conceptual framework analyzing Emotional Labor (adapted from Grandey 2000)

Furthermore, in the discussion of this model, Grandey (2000) focuses on the following major topics: (i) situational antecedents of emotional labour (e.g. customer interaction expectations, emotional events), (ii) stress and well-being as outcomes of emotional labor (including burnout 
and job satisfaction), (iii) work behaviours as outcomes of emotional labour (focusing on customer service performance and withdrawal behaviours), and (iv) personal and organizational factors affecting emotional labour (including personal characteristics related to emotional labor and organizational factors). I have placed in italics words which I believe are key to the conceptual exercise presented above, as these suggest an important allocation of focus not only to the emotional labor concept per se, but also to both the antecedents of emotional labour (e.g. situational factors and personal and organizational factors) and the results of emotional labour (e.g. stress and well-being and work behaviours).

Consequently, two issues concerning the model can be brought forward from Grandey's conceptual exercise:

1. The model mainly builds upon prior research in an attempt to bring the scattered literature together.

2. The model specifies: (a) the central concept, (b) its antecedents, and (c) what is produced, caused or affected/ effected.

The first issue is fairly self-evident as a literature review is an essential part of any scientific publication. Moreover, when trying to conceptualize a notion, a literature review allows the identification of the way, or various ways, in which the notion has been or could have been interpreted in the past. The second issue is somewhat most insightful and, moreover, goes well in the lines of this dissertation. Although it is important to identify the different interpretations of cost consciousness in prior literature, there are other aspects which could raise even more interest, for example, its determinants. The determinants of cost consciousness refer to the factors, activities, capabilities or happenings which could drive, create or support the emergence of cost consciousness in a particular system. Furthermore, an increase of cost consciousness should lead to a result or outcome, for there should be a reason explaining why cost consciousness is, or is not, increased or decreased in a system. Therefore, I propose the use of a framework which not only serves as a structured tool to synthesize the information regarding the interpretations of the notion itself, but also, and perhaps more importantly, identifies the determinants which drive cost consciousness in any system (e.g. an individual or an organization), and highlights the results or outcomes which could arise from increasing, or decreasing, cost consciousness in that system (Figure 4).

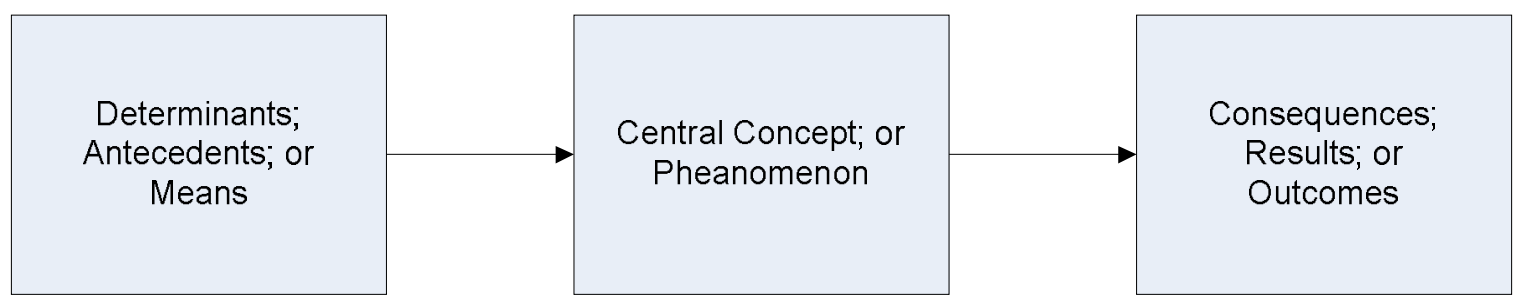

Figure 4. High level framework for understanding a concept or phenomenon

This framework could be used to provide a general analysis of various concepts or phenomena. For example, in an attempt to understand employee motivation, the existing literature does not only address the understanding of the employee motivation notion per se, but rather allocates substantial efforts on discussing the determinants and results of employee motivation. Examples of determinants of employee motivation are variable-pay programs, piece-rate pay plans, meritbase pay plans, bonuses, profit-sharing plans, employee stock ownership plans, skill-based plans, pension rights, material goods (André 2008, p. 115; Robbins and Judge 2008, pp: 99-106; M ullins 
2008, p. 174). Moreover, the results of increasing employee motivation could be positive, such as higher work motivation, high quality work performance, high work satisfaction, low absenteeism and turnover (Robbins and Judge 2008, p. 91; Mullins 2008, p. 177). However, increasing employee motivation could also result in negative outcomes, such as low performance, a decrease in job satisfaction, an increase in turnover and absenteeism, and avoidance of risk needed to develop innovation (Robbins and Judge 2008, p. 105; Greenberg 2010, p. 362).

Keeping the previous discussion in mind, this dissertation proposes the study of cost consciousness with the use of the framework presented in Figure 4; hence, three goals must be achieved. First, identify the definitions and most common interpretations of cost consciousness from prior literature. Second, gather the determinants of cost consciousness which have been previously evaluated or discussed. Third, collect the possible positive and negative results which arise from increasing cost consciousness in a system. The completion of this framework should not only serve as a tool for understanding the notion, but also to identify future research possibilities concerning cost consciousness.

In sum, this subchapter has provided an overview on three views on concepts highlighting the classical view as the one which this dissertation will take concerning the conceptual analysis. Furthermore, this subchapter has noted that the literature review on cost consciousness, from a management accounting perspective, will concentrate in identifying and inferring the determinants, consequences and possible definitions and interpretations which management accounting scholars have associated with the notion. However, as noted, this dissertation will be supported by illustrative research strategies which will be discussed in the following subchapter.

\subsection{Illustrative Research Strategies}

The results of this dissertation are supported by three illustrative research strategies: a survey, a set of interviews, and a longitudinal case study. Furthermore, integrating survey research and case studies can provide support in increasing certainty with respect to knowledge of a particular phenomenon (Figure 5) 


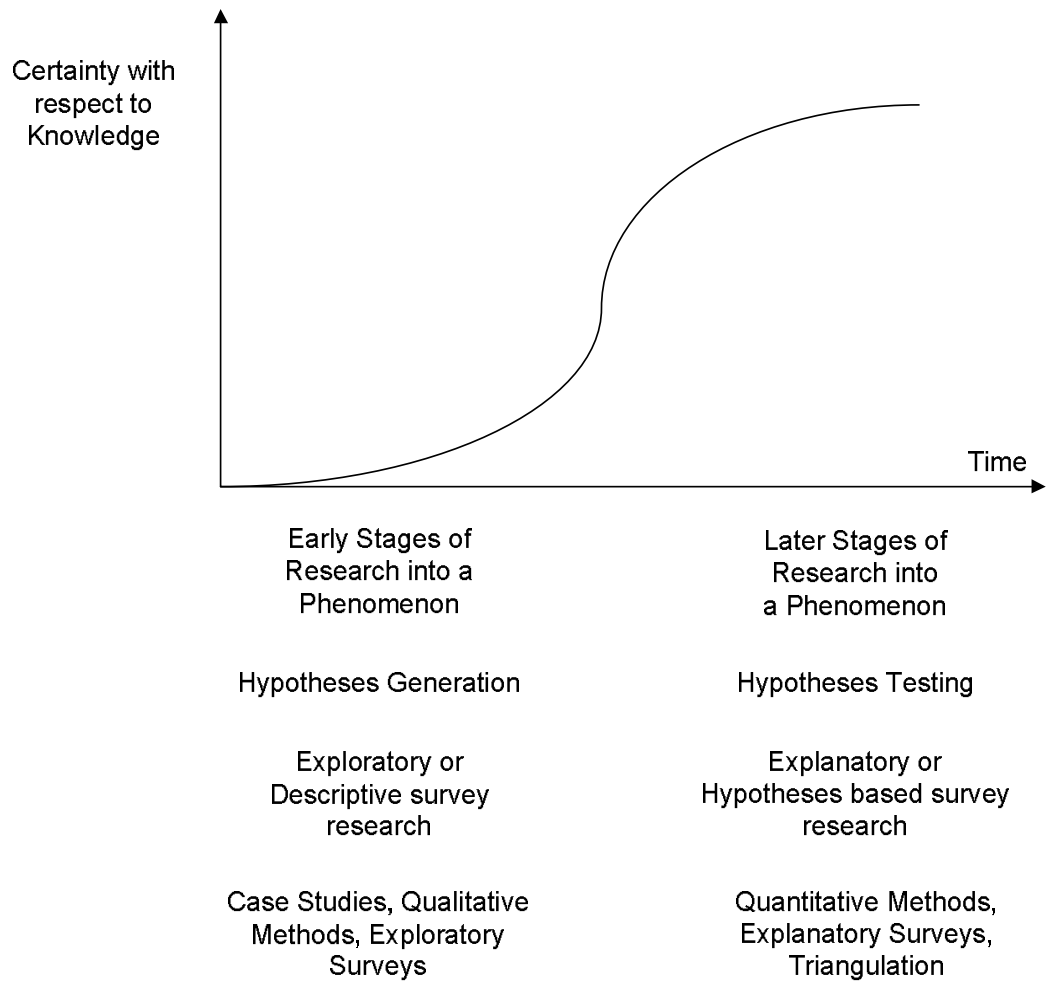

Figure 5. The maturity cycle of research (Malhotra and Grover 1998)

The survey, interviews and case study of this dissertation support the "early stages of research into a phenomenon" observed on the figure above. The results of these methodological strategies are expected to provide foundations for future research, which can support the "later stages", and may include quantitative methods, explanatory surveys, and triangulation.

\section{Survey Research and Interviews}

My personal involvement with the concept of cost consciousness finds its roots during the analysis of the results drawn from a survey conducted during the winter of 2007-2008. The survey aimed to evaluate the state of long term costing practices in Finland's manufacturing sector where almost 80 responses were collected. In addition to an overall evaluation of the state of long term costing practices, the study provided a new approach for the analysis of the costing capabilities of an organization or region.

Hence, the survey which is reviewed in this dissertation was not meant to illustrate the results of the conceptual or theoretical exercise as it was conducted before these exercises took place. However, in retrospective, the results of this survey do allow the illustration of some of the discussions which take place in the conceptual exercise. In addition, as the survey triggered my personal interest in the notion, I believe that it is important to include it in the discussions of the illustrative research strategies which support this work. Therefore, this section provides a brief overview of survey research.

There are many types of data elicitation processes which include opinion surveys, political polls and survey research; the latter being conducted to advance scientific knowledge or develop theory and has the three distinct characteristics (Malhotra and Grover 1998). First, survey research involves collection of information by asking people for information in some structured format through mail questionnaire, telephone interview, or face-to-face interviews. Depending 
on the unit of analysis, the individuals surveyed could be representatives of themselves, their project, their expertise, or their organization. Second, survey research is usually a quantitative method that requires standardized information in order to define or describe variables, or to study relationships between variables. Finally, information is gathered via a sample, which is a fraction of the population, in an attempt to generalize findings from the sample to the population.

According to Kerlinger (1986, p. 410) survey research "studies large and small populations by selecting and studying samples chosen from the populations to discover the relative incidence, distribution, and interrelations of sociological and psychological variables". Moreover, Forza (2002) states that researchers commonly distinguish between three types of survey research:

1. Exploratory survey research. The objective of this type of survey research is to familiarize and gain preliminary insight into a phenomenon aiming to provide foundations for future more in-depth surveys. Therefore, exploratory survey research takes place, and is of great importance, in the early stages of research into a phenomenon. Moreover, it can help determine the concepts to be measured in relation to the phenomenon of interest and in later stages of research it can not only help uncover preliminary evidence of association among concepts but also allow the exploration of the valid boundary of a theory.

2. Confirmatory (or theory testing or explanatory) survey research. This type of research takes place once the articulation of the knowledge of a phenomenon has taken place in a theoretical form using well-defined concepts, models and propositions. The main objectives of confirmatory survey research are to find causal relationships among variables, test the adequacy of the concepts developed in relation to the phenomenon and of the validity of boundary of the models.

3. Descriptive survey research. This type of survey research has been considered by some authors as "another type of exploratory research" (Malhotra and Grover 1998). The main objective of descriptive survey research is to understand the relevance of a certain phenomenon and describe the distribution of the phenomenon in a population, hence assert facts.

Furthermore, Forza (2002) recalls four elements of the conceptual model which a researcher should establish prior to starting theory testing survey research:

i. Construct names and nominal definitions: clear identification, labels and definitions of all the constructs considered relevant

ii. Propositions: presentation and discussion of the role of the constructs (independent, dependent, intervening, moderating), the important linkages between them, and an indication of the nature and direction of the relationships (especially if available from previous findings)

iii. Explanations: a clear explanation of why the researchers would expect to observe these relationships and, eventually, linkages with other theories

iv. Boundary conditions: definition of conditions under which the researcher might expect these relationships to hold; it includes the identification of the level of reference of the constructs and their statements of relationships (i.e. where the researcher might expect the phenomenon to exist and manifest itself: individual, group, function, or organization). 
Given the abstract nature of cost consciousness and the little knowledge supporting its understanding, an exploratory survey research could be considered as the obvious and most rational choice for gaining preliminary insight into what is, and perhaps more importantly what constitutes cost consciousness. However, the survey research which is presented in this work is a descriptive survey research whose aim was to describe the state of long-term costing practices in the Finnish manufacturing sector.

\section{M ethodology of the Survey Research}

During the late autumn of 2007, a questionnaire focusing on the costing practices associated with fixed asset investments - using International Financial Reporting Standards (IFRS) terminology we refer to non-current tangible items such as production machines, tools, vehicles, and so on - was developed for conducting the survey. The questionnaire was composed of 54 questions that were intended to solicit information regarding the types and the targets of the acquisitions, and cost monitoring and forecasting practices related to these acquisitions. Moreover, the questionnaire was composed of six main sections: (1) General Overview, (2) Target-Product (the most important category of fixed asset acquisitions), (3) Costing in acquisition Phase, (4) Costing during target-product's active use, (5) Disposal, and (6) Evolution and Challenges of Life Cycle Costing (LCC). The main objective of the first section of the survey was to obtain a general view of the target audience. In the second section, the respondents were asked to describe a single product (the target-product), which they would use as reference for the remaining parts of the survey. The third section of the survey was intended to solicit a description of how life cycle costs pertaining to the target-product were estimated in the acquisition phase of that product. The fourth section focused on the life cycle cost estimation during the target-product's active use. The fifth section analyzed certain aspects of the disposal stage of the target-product. The survey concluded by asking respondents about the importance of cost consciousness for the development of an organization. Furthermore, the sample of respondents was drawn from private companies belonging to the manufacturing industry. Teknologiateollisuus r.y. ${ }^{7}$ provided the contact information of the financial department managers or directors of those organizations. The survey was conducted in January-February 2008. The questionnaire was published with the use of the Webropol tool on the Internet and the invitation to answer it was sent to almost 800 companies, first by means of traditional mail and then by email with a link to Webropol tool. The invited respondents were given about two weeks to respond. A relatively low number of 76 responses were received after one reminder e-mail. The response rate, however, cannot be calculated by simply dividing 76 by 800 . Based on automatic e-mail responses we estimate that approximately 400-500 managers were actually reached. The estimated sample size would lead us to a response rate between $15-19 \%$.

The interviews: Asking about employees' perception on cost consciousness

The case of the Metso operations in China was developed during the year 2011 while I was a member of the CHINDICA (China - India Initiative for Competitive Adaptation) research project created as a "multidisciplinary and multinational study of strategic challenges in global

\footnotetext{
${ }^{7}$ This organization is The Federation of Finnish Technology Industries, which, according to their own website http://www.teknologiateollisuus.fi, focuses on improving the competitiveness of their partners in Finland.
} 
management in the fast changing economy" ${ }^{\prime 8}$. The project was conceived in cooperation between the Center for Research on Information, Customer and Innovation M anagement (CIRCMI) of the University of Tampere, the Cost Management Center (CMC) of the Tampere University of Technology, the Antai College of Economics and Management (ACEM) of Shanghai Jiao Tong University, and the Management Development Institute, Gurgaon. The general focus of the CHINDICA project is twofold: (1) to collect interview data of management challenges in fast changing global markets; and (2) to analyze the opportunities and challenges that the great areas of Shanghai in China and Delhi in India can offer to Finnish companies. Particularly, I was involved in the realization of interviews in the city of Shanghai to evaluate the internationalization process of various Finnish companies operating inside and in the region surrounding the city.

The results of the survey, described in the previous subsection, allowed me to infer that the interviewees seemed to share some assumptions with respect to what cost consciousness could mean. This, furthermore, triggered my interest on cost consciousness. Hence, through the CHINDICA project, I conducted a set of interviews allowing me to question a group of employees about what they believe cost consciousness involved. Although CHINDICA studied various Finnish companies, the interviews supporting this work were mainly focused on one particular organization (i.e. Metso Corporation). Metso is a multinational Finnish supplier of industrial machinery and systems which has been traditionally associated with the paper-manufacturing industry. Furthermore, the interviews focused on reviewing the interpretations and connotations of cost consciousness among employees of $M$ etso and $M$ etso suppliers. In general, interviewees were asked about how they interpreted cost consciousness, and, perhaps more importantly, how they believed cost consciousness could be increased in their company. The prioritization on the drivers of cost consciousness (when compared to the actual interpretation of the construct) is clearly aligned with the methodology used for analyzing the results of the literature review on cost consciousness from a management accounting perspective where focusing on how cost consciousness may be driven deviates from the conceptual challenges which arise from trying to define the construct. Furthermore, some of the results of these interviews supported the findings of the literature review. It is important to highlight that the aim of the interviews in this work is not to validate the proposed formulation, but to serve as an example on how empirical findings may guide the conceptualization process of a notion.

In sum, the survey research and interviews which are described in this dissertation were not meant to validate the results of the conceptual and theoretical exercises conducted in this dissertation. However, in retrospective, the results of these two methodological strategies provide illustrative support to the results from the conceptual and theoretical exercises.

\section{Case Study Research}

The results of the theoretical exercise of this dissertation are illustrated with one case study. This section discusses various issues related to case study research, beginning with general issues (definitions, advantages, disadvantages) and turning at the end of the section to more specific discussions (design and process). In contrast to the distinct characteristics of survey research presented in the previous section, "case studies are not usually quantitatively oriented, the variables are often not predefined, and such studies involve examination of a phenomenon in depth within their natural setting, thereby precluding any attempt at generalization" (Malhotra

\footnotetext{
${ }^{8}$ This quote was obtained from an internally distributed presentation by Professor Mikko Ruohonen and Dr. Lea Ahoniemi, both from the University of Tampere.
} 
and Grover 1998). In addition, case study research actually relies (commonly) on qualitative methods to generate data, being interviews one of the most common techniques (Gummesson 1993).

The question 'What is a case study?' has proven to dodge the straightforward answer which I, admittedly, initially expected. In fact, before providing any definition which could satisfy the curiosity of most readers, I will refer Gerring (2004) who asserts that case study "is a definitional morass" as it might mean (a) that its method is qualitative, small-N; (b) that the research is ethnographic, clinical, participant-observation, or otherwise "in the field"; (c) that the research is characterized by process-tracing; (d) that the research investigates the properties of a singlecase; or (e) that the research investigates a single phenomenon, instance, or example. M oreover, case study research does not only present conceptualization obstacles, but also other more practical issues. For example, McPhee (1990) argues that comparative analysis integrating findings of numerous studies by multiple researchers or teams of researchers is a central problem in social science.

Regardless of the conceptual difficulties, definitions of case study and case study research are available. Gerring (2004, p. 342), after acknowledging the "definitional morass" of the construct, proposes to define a case study as "an intensive study of a single unit for the purpose of understanding a larger class of smaller units". Moreover, Yin (1994, p.13) defines a case study as "an empirical enquiry that investigates a contemporary phenomenon within its real life context, especially when the boundaries between the phenomenon and the context are not clearly evident". With respect to the objectives of case study research, Woodside (2005) highlights the importance of acquiring a deep understanding of the actors, interactions, sentiments, and behaviours occurring through time for a specific process. Additionally, Jaspers (2007) argues that the main application for case study research in management research is theory building.

Finally with respect to the general issues concerning case studies, one should be aware of the advantages and disadvantages which case studies provide. According to Eisenhardt (1989), there are both strengths and weaknesses of theory building from cases. The strengths include a likelihood of generating novel theory which is likely to be empirically valid. However, this might also lead to a theory which is too complex, narrow or idiosyncratic. Moreover, Gerring (2004) argues that case studies are generally more useful (i) when inferences are descriptive rather than causal, (ii) when propositional depth is prized over breadth and boundedness, (iii) when (internal) case comparability is given precedence over (external) case representativeness, (iv) when insight into causal mechanisms is more important than insight into causal effects, (v) when the causal proposition at issue is invariant rather than probabilistic, (vi) when the strategy of research is exploratory, rather than confirmatory, and (vii) when useful variance is available for only a single unit or a small number of units. Therefore, as this research is reasonably of exploratory nature, a case study could be considered to be useful following Gerring's argumentation.

Case Study: Institutionalization of cost consciousness in Kopla Ltda.

Kopla is one of the brands of Punto Franco Agrocommodities (PFA), a small agribusiness company focusing on planting, harvesting, processing, and marketing various food products. Although PFA owns various businesses and brands, this case study focused solely on the activities concerning Kopla. The company's headquarters are situated in the city of Armenia which sits 
right in the center of Colombia's "Coffee Axis" ${ }^{\text {" }}$ Kopla purchases four basic food products (cassava, plantain, potato, and arracach ${ }^{10}$ ) and processes these into different finished and semifinished food products (e.g. shredded cassava, peeled and cut plantain, potato "French-fries", frozen arracacha cut in pieces). All of these food products, one could reasonably argue, are part of the common diet of many Colombians. For example, plantain chips are sold in Colombian supermarkets and grocery stores in an analogous way as potato chips are sold in countries like Great Britain, Finland, and the United States. However, Kopla's main target is not the endconsumer but other businesses such as restaurants, hotels, and companies that purchase their products to process them further aiming to reach end consumers. This case study was used for two main purposes. First, it allowed the study of an environment where key actors took measures to institutionalize cost consciousness. Second, it allows illustrating the use of the proposed formulation presented in this work for analyzing the elements which may interact in the institutionalization of cost consciousness.

The conduction of this case study took place over a period of four years; from 2008 to 2012. Initial contact occurred during the latter part of 2008 when I was asked to conduct a process and management control audit for the company. Although this initial task fell outside my academic profession, I quickly understood the potential that it had for my own research. Therefore, after various interviews and meetings by phone and via email, the first visit to Kopla took place in June 2009 during one week attending their offices and processing plant. The main focus of this visit was to understand the administrative and non-administrative activities and processes involved in the revenue (and receivables) and purchasing (and payables) cycles of the company. Following my own experience when working as a Systems and Process Assurance auditor for PricewaterhouseCoopers, I mapped these cycles using Flow Chart Diagrams where I took notice of those activities or processes where a financial control would take place. In this way I was able to identify the systems and procedures which the company uses to track and monitor its financial information, including costs. The second visit to Kopla was conducted in July 2010 where I was invited to attend a board meeting where the head of each division (general management, production, purchasing, quality control and finance) gave a presentation detailing the major events of the past year and the objectives and possible obstacles for the future. The third visit to Kopla was held on June 2011 when, during four days, I conducted several interviews and participated in one board meeting where the results of a new costing system were being reviewed. The fourth visit was held on December 2011 where the major owner of the company was interviewed about the development of the previously introduced costing system. Finally, various interviews were conducted on 2012 via phone calls and email.

\section{Reliability and Validity of Results}

This section provides a discussion on the concepts of reliability and validity; what they mean, and how this work provides reliable and valid results. Before reviewing these concepts, however, it is important to provide an overview on operationalization referring to the conduction of some kind of operation to make a concept more concrete. In the case of quantitative research,

\footnotetext{
${ }^{9}$ This is a literal translation of "Eje Cafetero", the region comprising the departments of Quindío, Caldas, and Risaralda. Armenia is the capital of Quindío.

${ }^{10}$ Arracacha (arracacia xanthorriza) is a perennial plant originally domesticated in South America and currently cultivated mainly across South and Central America, the Caribbean. The principal economic product of the arracacha are its storage roots which are used for culinary purposes and can be considered as a staple food in many countries (Hermann, 1997)
} 
operationalization is commonly conducted through measurement (e.g. the measurement of temperature). Measurement is viewed as the process of linking abstract concepts to empirical indicants (Carmines and Zeller, 1979). Scholars use measurement to focus on the relationship between observable empirically based indicators and unobservable concepts. The strength of this relationship, however, varies, and this variation clearly affects the way in which the analysis of the empirical indicators leads to useful inferences about the relationship between the concepts. Hence, assessing the strength of how a particular empirical indicator represents a theoretical concept is of great importance. This assessment, furthermore, can be conducted by evaluating two basic properties of empirical measurements: reliability and validity. Both properties have a definite positive connotation therefore making reliable and valid measures a desirable objective in any research endeavor. Furthermore, there are other kinds of operationalization mechanisms. In the case of qualitative research, operationalization is conducted in great part through observations in an attempt to, for example, develop concepts further.

However, before discussing what reliability and validity are, it is important to spare some discussion on measurement error. M easurement error, which can be identified as either random or non-random, has commonly been recognized as a major problem throughout the social sciences as any measure often reflects not only a theoretical concept of interest but also measurement error (Bagozzi et al. 1991). First, random error refers to those chance factors that confound the measurement of any phenomenon. Carmines and Zeller (1979) argue for two important points which must be taken into account when considering random error: (a) indicators always contain random error to a greater or lesser degree; and (b) the effects of random error are totally unsystematic in character. Moreover, the authors also point to a high number of sources of random measurement error in social sciences. For example, in survey research, random errors include interviewer fatigue, errors due to coding and ambiguous instructions. In addition, the second type of error is non-random error (also referred to as systematic error) refers to a systematic biasing effect on measuring instruments.

Reliability

The first property of empirical measurement which will be discussed is reliability. In this discourse, the focus is on how reliable the gathered empirical data is. This, furthermore, is clearly related to the reliability of the measuring device or pursued strategy. Reliability concerns the extent to which any measuring procedure (e.g. an experiment) yields the same result on repeated trials (Carmines and Zeller 1979). It is important to note that total reliability is not possible because repeated measurements never exactly equal one another therefore making unreliability always to be present. However, repeated measures of the same phenomenon would tend to provide a degree of consistency, resulting in a more reliable measurement of that phenomenon. Therefore, reliability is the tendency towards consistency in repeated measurements of a particular phenomenon.

With respect to measurement errors, there is an inverse relation between the degree of reliability of a measuring instrument and the amount of random error. In the case study research, a higher amount of random error (e.g. a fatigued interviewer, ambiguous instructions) would probably result in less reliable data. M oreover, non-random error does not detract from a measure's reliability as all of the error in a measure could be nonrandom and still would not affect the measure's reliability (Carmines and Woods 2005). 
Measuring reliability has been a long tradition in quantitative research. However, in qualitative research, it has been regarded as misleading (Golafshani 2003). Golafshani states that to ensure reliability in qualitative research, examining trustworthiness is a crucial step. The author, however, provides few clues on how to purse trustworthiness while recalling on authors who claim that reliability is irrelevant in qualitative research (Stenbacka 2001) and those who state that by demonstrating validity, reliability is established (Lincoln and Guba 1985). Golafshani follows Lincoln and Guba by stating "Now, the question which remains to be answered is 'How to test or maximize the validity and as a result the reliability of qualitative study?'" (p. 602). Hence, following Golafshani, the following subsection discusses the notion of validity.

\section{Validity}

Although reliability is a clearly important property of empirical measurement, as Carmines and Zeller (1979) clearly state, an indicator must be more than reliable if one expects to obtain accurate representation of an abstract concept, thus bringing validity, the second property of measurement, into the discussion. Validity refers to the extent to which an indicator of some abstract concept measures what it purports or intends to measure (Carmines and Woods 2005). According to Bagozzi et al. (1991), because measurement errors provide potential threats to the validity of research findings, it is important to validate measures and disentangle the distorting influences of these errors before testing theory. M oreover, validation of some kind is a necessary condition for any research endeavor to be taken seriously (Lukka and M odell 2009).

Similarly to reliability, obtaining a perfectly valid indicator is not possible, thus suggesting that validity is a matter of degree and not an either-or type of property. However, when compared to reliability, validity is considered to be more theoretically demanding as it raises the question "valid for what purpose?" (Carmines and Zeller 1979). Moreover, there are other clear differences between the two properties. While reliability focuses on the extent to which empirical indicators provide consistent results across repeated measurements; validity concerns the crucial relationship between concept and indicator (Carmines and Zeller 1979). Furthermore, as it was mentioned when discussing reliability, an indicator which is reliable does not necessarily mean that it is valid (at least in the case of quantitative research). One final difference worth noting between the two properties is that while random errors are central whenever discussing reliability, in the case of validity, non-random errors prevent indicators from representing what they are intended to; thus giving rise to invalidity (Carmines and Zeller 1979). In a way, method variance is strictly a kind of non-random or systematic error (Bagozzi et al. 1991).

Concepts of validity, as abstract as they can be, have had a substantial impact upon how researchers think about their work (Calder et al. 1982). Furthermore, numerous types of validity exist (Carmines and Woods 2005) but the following seem to be of common discussion in social sciences (Straub 1989, Yin 1994, Carmines and Woods 2005): content validity, criterion-related validity, construct validity, internal validity, and external validity (Carmines and Zeller 1979, Straub 1989, Yin 1994, Carmines and Woods 2005). Content validity depends on the extent to which an empirical measurement reflects a specific domain of content. It answers the question "does the indicator adequately and comprehensively represent what it is supposed to measure?" Moreover, scholars can obtain content validity through a successful conduction of two steps: (i) the specification of the entire domain of content that is relevant to a particular measurement situation, and (ii) selecting or constructing the specific indicators that are used in the measure. With respect to this dissertation, content validity presents important limitations. According to (Carmines and Woods 2005) when dealing with abstract concepts (e.g. cost consciousness) it is 
difficult to establish concurrence about a domain of content relevant to the phenomenon because most theoretical concepts in the social sciences have not been described with the required exactness. This, partly, forces scholars to provide a plausible rationale for accepting a version of what constitutes the appropriate domain of content and for establishing that the indicators in the measure have been satisfactorily sampled from that domain. As this is not an easy task, to say the least, content validity is not the preferred way to assess validity in social sciences. Moreover, criterion-related validity concerns the correlation between a measure and some criterion variable of interest. This assessment can be conducted once a direct measurement to evaluate the criterion is considered. In addition, there are two types of criterion related validity: present and predicative. In the former, concurrent validity can be assessed by correlating the measure and the criterion at the same point in time. In the case of the latter, a future criterion is correlated with the relevant measure. Also, and similarly to content validity, criterion-related validity has limited usefulness in social sciences where the more abstract the concept is, the more difficult it is to find an appropriate criterion for assessing its measure. In addition, construct validity is defined as the extent to which an operationalization measures the concept it is supposed to measure (Bagozzi et al. 1991). In other words, it tests the extent to which a measure reflects a theoretical concept of interest (Dedehayir 2010). This type of validity is theory driven and it is particularly important "when there is no universal agreement concerning the domain of content for the phenomenon, and no relevant criteria" (Carmines and Woods 2005). In this case, a researcher uses theory to formulate predictions about the existence, direction and extent of relations among empirical indicators of different theoretical concepts. A measure is said to be construct valid if the empirical outcomes are consistent with the theoretical predictions. Given its embracement to theoretical exercise, construct validity is more pertinent in the social sciences when compared to the other two types. According to Yin (1994, p.35), internal validity establishes a causal relationship, whereby certain conditions are shown to lead to other conditions, as distinguished from false or incorrect relationships. Campbell and Stanley (1969) argue that internal validity is the basic minimum without which any experiment is un-interpretable by, for example, asking 'did in fact the experimental treatments make a difference in this specific experimental instance?' This type of validity serves strictly explanatory or causal studies and should be avoided in descriptive or exploratory studies. However, although this dissertation serves as an exploratory study, internal validity is important for this work as I am interested in analyzing the causal mechanisms within my case study. Finally, external validity examines whether or not an observed causal relationship should be generalized across different measures, persons, settings and times (Calder et al. 1982). In other words, external validity asks the question of generalizability by asking 'to what populations, settings, treatment variables, and measurement variables can an effect be generalized?' (Campbell and Stanley 1969). According to Yin (1994) external validity deals with the problem of knowing whether one can generalize the findings of a study beyond the immediate case study.

Furthermore, it is important to take note of the controversy with regards to the support which external validity provides to theoretical research. While one view asserts that external validity must be a priority in theoretical research, others, including Calder et al. (1982), argue that external validity is not necessary for achieving rigorous theory test and may be sacrificed in favor of addressing threats to internal and construct validity.

Moreover, Maxwell (1992) highlights the critique that existing categories of validity (e.g. criterion-related validity, internal validity, and external validity) are based on assumptions underlying quantitative and experimental research. However, not all authors agree. For example, Johnson (1997) argues that internal validity and external validity, among other types of validity, 
are important for qualitative research. Furthermore, while arguing that "understanding is a more fundamental concept for qualitative research than validity" (Maxwell 1992, p. 281), the author reviews five types. First, descriptive validity deals with matters of descriptive accuracy. These may be divided between primary descriptive validity (i.e. what the researcher reports having seen or heard), and secondary descriptive validity (i.e. accounts from highly complex inferences). Second, interpretive validity is concerned with the meanings that people associate with objects, events and behaviours. Maxwell (p. 289) explains that "while the terms involved in descriptive validity can be either etic or emic, interpretive validity necessarily pertains to aspects of an account for which the terms are emic". Third, theoretical validity addresses the theoretical constructions brought and developed during the study by the researcher. M oreover, theoretical validity is concerned not only with the validity of the concepts as they are applied to the phenomena, but also to the relationships between concepts. Fourth, generalizability is related to the extent to which the account of a particular situation, event, or population can be extended to other settings. Furthermore, when conducting case studies, Yin (1994) recommends aiming toward analytic generalization, rather than focusing on statistical generalization. In addition, generalizability is composed of two aspects. The first aspect is internal generalizability referring to the generalization "within a community, group, or institution suited to persons, events, and settings that were not directly observed" (p. 293). The second aspect, external generalizability, refers to "generalizing to other communities, groups, or institutions". Fifth, evaluative validity "involves the application of an evaluative framework to the objects of study, rather than a descriptive, interpretive, or explanatory one" (p. 295). Maxwell highlights that both external generalizability and evaluative validity are not central to qualitative research in the same way as the other types are.

Hence, the results of this dissertation are, naturally, more in line with the types of qualitative related validity formulated by Maxwell. In particular theoretical validity, due to its objective on addressing concepts and the relationship between these, seems to be especially relevant. However, the case study and interviews are also subject to descriptive and interpretive validity given that the data is gathered, on the one hand, from what the author hears and sees during the interviews and case studies; and, on the other hand, the meanings and interpretations which the author must attribute in the course of conducting these methodological strategies.

Moreover, Morse et al. (2002) call for the use of techniques of verification to increase validity in qualitative research. Verification techniques include: ensuring methodological coherence, using an appropriate sample, collecting and analyzing data concurrently, thinking theoretically, and theory development. With respect to theory development, the authors state that "theory is developed through two mechanisms: (1) as an outcome of the research process [...] and (2) as a template for comparison and further development of the theory" (pp. 18-19). Furthermore, according to Johnson (1997) there are thirteen strategies used to promote validity in qualitative research including the researcher acting as a "detective" (i.e. the researcher searches evidence about causes and effects). With regards to the techniques mentioned above, this dissertation follows a sound theory development technique as the findings of the literature review are used to inform the theoretical exercise which then serves as a kind of template or formulation for future development of our understanding on the institutionalization of cost consciousness. Moreover, the rigorous activity of finding drivers and results of cost consciousness reflects my own role as a "detective". 
Finally, I would like to invoke Lukka and Modell (2009) who, in addressing the emerging "crisis of validity" in interpretative management accounting research, suggest two strategies for going forward in validating this type of research:

"One strategy [...] is to mobilise theoretically informed explanations which may not yet be fully accepted by the wider research community by appealing to the need to give voice to the "Others" who remain under-represented by established theories [...] An alternative strategy [...] is to take [firmly entrenched theories] as a rather general starting point and rely heavily on rich, emic accounts whilst leaving explanations somewhat open-ended and thus affirming the messy and often paradoxical nature of life in complex organisations" (Lukka and M odell 2009, p. 14)

Within this respect, the illustrative case study in this dissertation adopts, to a certain extent, the second strategy. Although the amount of visits to the case company was limited given the geographical distance, my visits were very intense with respect to the amount of time which I spent with the employees and director of the company.

In sum, this subchapter has reviewed the concepts of validity and reliability. Validity and reliability seem to be more central in works focused on empirical research. However, internal validity seems to be important for this work as a considerable part of the conceptual and theoretical exercises are based on analyzing causal relationships. Nevertheless, this subchapter has also reviewed types of validity which are argued to be more proper whenever conducting qualitative research. In particular, theoretical validity might be an important type to consider when evaluating the results on how organizational elements interact in the process of institutionalizing cost consciousness. In addition, descriptive and interpretative validity will probably be an issue when analyzing the interviews and the illustrative case study. The reader might wonder why it is important to provide such an amount of focus on the types of validity which are more central in quantitative research endeavors when this particular dissertation is of a qualitative character. The author believes that as validity and reliability are such important and central concepts to any type of research; and, furthermore, as these concepts have had a long tradition in quantitative research, it is important to provide an overview of the main types of validity central to quantitative studies.

Summarizing this chapter, this dissertation serves as an exploratory study composed of conceptual and theoretical exercises where the conceptual development of cost consciousness is approached by a literature review identifying the drivers, interpretations/definitions, and results which management accounting scholars have associated with the notion. Furthermore, the results of the theoretical exercise will be illustrated by a case study. 


\section{Cost Consciousness from a Management Accounting Perspective}

In this chapter I will discuss the linguistic composition of cost consciousness; present the drivers, definitions, interpretations, and results associated to the notion; and synthesize these associations by also taking into account the results of a survey and a set of interviews. Therefore, this chapter constructs, to a great extent, the first contribution of this dissertation (i.e. shedding light on the possibilities for future research related to cost consciousness by conducting a thorough literature review identifying the drivers, interpretations, and results associated to the notion from a management accounting perspective).

As mentioned in the introduction of this study, cost consciousness's linguistic composition allows one to reasonably argue that while it's linguistic head (i.e. consciousness) provides the compound with a broad conceptual realm, the notion's modifier (i.e. cost) narrows and restricts this realm. Moreover, this type of linguistic composition is shared by other notions such as market orientation which is believed to represent "superior skills in understanding and satisfying customers" (Day 1994, p. 37). In addition, market orientation has also been defined as "the business culture that most effectively and efficiently creates the necessarily behaviours for the creation of superior value to customers" (Narver and Slater 1990, p. 20). Hence, while market orientation's linguistic head (i.e. orientation) provides it with a broad enough conceptual realm to embrace other abstract (and difficult-to-define) notions such as skills and culture; the concept's linguistic modifier (i.e. market) narrows these descriptions on aspects like customerneeds. Therefore, one could reasonably argue that market orientation's linguistic composition allows it to taint these skills and culture in a particular manner which may serve research on, for example, marketing studies. Furthermore, these interpretations of market orientation have fueled multiple research endeavors and publications focused, for instance, on scales to measure market orientation, discussions on related managerial implications, and, naturally, models to conceptually develop the notion. As a result, the understanding of skills and organizational culture tainted with market orientation becomes more refined as the research on the notion continues. Therefore, one can reasonably argue that the notion's broad but restricted linguistic composition translates into a flexible conceptual vehicle allowing scholars to describe other concepts like organizational skills or business culture in a manner which becomes more detailed and comprehensive as more research on the notion is conducted. However, as the results of this chapter will indicate, management accounting academics have not yet taken advantage of the potential which may lie within cost consciousness.

Hence, given the relevancy of cost consciousness's linguistic composition, subchapter 3.1 reviews the concepts of consciousness (i.e. the notion's linguistic head) and management accounting systems (a topic intricately related to cost, the notion's linguistic modifier). In addition, the introductory and methodological chapters discussed how the conceptual development of a notion may be approached by conducting a literature review identifying the antecedents (i.e. drivers), interpretations, and results which have been associated to the concept. Therefore, subchapter 3.2 presents and discusses the drivers, interpretations, definitions, and results which management accounting scholars have associated with cost consciousness in prior studies. Furthermore, the conceptual exercise is also supported by the findings from two methodological strategies (i.e. a survey and a set of interviews). Hence, subchapter 3.3 discusses and analyzes these findings. However, it is important to note that the survey and the set of interviews were not intended to validate the results of the literature review of cost consciousness from a management accounting perspective. Finally, the results of the literature review and the analysis 
from the methodological strategies are synthesized in subchapter 3.4 where the main results of the conceptual exercise are analyzed.

\subsection{Linguistic Composition: Consciousness \& Management Accounting Practices}

Cost consciousness's linguistic composition takes an important role in justifying the conceptual development of the notion by highlighting the notion's broad but restricted conceptual realm. Barker and Szpakowicz (1998) explain that a head noun accompanied by a noun premodifier is often referred to as a noun compound which, syntactically, acts as a noun. Moreover, linguistic compounds can be distinguished between two classes, endocentric and exocentric (Clackson 2002, Bauer 2008). On the one hand, the endocentric compound denotes something which is a hyponym of one of its elements. For example, a desktop computer is a type of computer, a teacup is a type of cup, and a door-knob refers to a particular kind of knob. On the other hand, exocentric compounds are somewhat more problematic as they have a different denotation and distribution to their individual members. For example, bird brain refers to a person, butterfingers is a candy bar, and blackout is considered as exocentric because there is no noun involved with the notion.

As cost consciousness is composed by two nouns, and acts syntactically as a noun, then it can be considered as a noun compound. Moreover, although it is not clear what cost consciousness denotes, one can reasonably argue that a possible denotation does not fall completely outside of the denotations of its elements as in the cases of bird brain or butterfingers. Furthermore, one could reasonably argue that cost consciousness reflects a type of consciousness rather than a type of cost. Hence, cost consciousness seems to represent a type of endocentric compound where consciousness acts as the linguist head, and cost acts as the modifier of the compound.

In an attempt to explore further the linguistic composition of cost consciousness, this subchapter provides, first, an overview on the notion of consciousness. Moreover, given the triviality of the concept of cost, a following section will focus on discussing management accounting systems, which are used in some cases to, for example, control costs; and, furthermore, have gathered a great deal of attention from management accounting scholars. Given that this dissertation takes a management accounting perspective, the revision of a central concept as such is of great importance.

\section{On Consciousness}

A compound's linguistic head contains the basic meaning of the whole compound. Therefore, one may argue that consciousness contains the basic meaning of cost consciousness.

Although Aristotle's discussion of perceiving that we perceive does not include the word consciousness, it has strong similarities with contemporary debates about this abstract notion (Caston, 2002). Furthermore, two thousand years later after Aristotle, Scott $(1918$, p. 1) would argue the following: 
Every one knows in a general way what consciousness is, but when we try to define it we encounter two formidable difficulties. One is caused by the various uses of the term [...] The other difficulty belongs to the nature of that which is to be defined.

Scott (1918) provides an explanation on why defining consciousness is so challenging. In doing so, the author argues that without consciousness there could be neither mental life nor knowledge of mental operations as consciousness is a primary fact. Moreover, as a primary fact, it is not possible to assign to it a formal definition; hence, the author suggests three methods which may be employed to express its meaning including: (i) noting instances of its presence or absence; (ii) defining it by its denotation by enumerating its different kinds: as sensation, perception, memory, imagination, thought, emotion, desire, will; and (iii) comparing consciousness with a related word such as awareness. More recently, Dennett (2001) would state that consciousness represents a hard task for anyone attempting to explain what it is. Therefore, it seems that the challenges related to understanding consciousness have accompanied humanity for ages.

Despite the seeming impossibility of defining consciousness, the Oxford Dictionary of English (2006) provides two definitions: (i) "the state of being aware of and responsive to one's surroundings"; and (ii) "a person's awareness or perception of something". Moreover while awareness is defined as "having knowledge or perception of a situation or fact"; perception is defined as (a) "the ability to see, hear, or become aware of something through the senses"; and (b) "the way in which something is regarded, understood, or interpreted". It appears, therefore, that consciousness is defined by recalling awareness and perception; while awareness is defined by referring to perception; and perception as an ability or 'way'. At the end, the three concepts remain arguably abstract. Hence, the dictionary provides very limited guidance in understanding the notion. Within this respect, Scott $(1918$, p. 2) states that consciousness and awareness are not exactly synonymous as awareness carries a broader sense "often having a more outward meaning than consciousness has. Awareness is either mediate or immediate, while consciousness is always immediate".

Despite the conceptual difficulties which the consciousness notion sets forward, Flanagan (1992), for example, has taken to the challenge of understanding consciousness taking a firm stance against those who have tried to quine the term ${ }^{11}$. He defends this position by stating, among other reasons, that even if all the extant conceptions of consciousness deserve the quining treatment, the phenomenon of consciousness, however poorly understood, is robust enough to keep its ground against those who try to dismiss it:

The words 'conscious' and 'consciousness are invoked relatively infrequently in ordinary causal discourse. But even when they are, they cause little trouble in communicative flow [...] our everyday mental discourse shows no signs that some single, overly simplistic concept of consciousness obstructs our ability to pick out the multifarious array of types of conscious mental events that figure in the overall economy of human mental life [...] I do think that there is something that binds all the various concepts of consciousness and that cannot be given up. (Flanagan 1992, pp. 30-31)

Furthermore, consciousness is also a topic of interest in structuration and institutional theories which support the theorization exercise of this dissertation in later chapters. For now, in an

11 'To quine', a verb in honor of W.V.O. Quine, means to deny resolutely the existence or importance of something seemingly real or significant, for example, the soul (Flanagan, 1995). 
attempt not to deviate too much from the topic, I will only mention that, according to Giddens (1984), there are two main types of consciousness: discursive and practical. While the former refers to the knowledge and behavior which can be discursively explained, practical consciousness refers to the tacit knowledge that is skillfully applied in the day-to-day interactions, but which the actor is not able to formulate discursively. Moreover, practical consciousness is considered to be fundamental for structuration theory as it explains a considerable (if not, the largest) amount of our daily actions and reconstruction of structures and societal institutions.

Finally, Dennett (2001) argues that "[as] the Decade of the Brain (declared by President Bush in 1990) comes to a close, we are beginning to discern how the human brain achieves consciousness [...] there is enough common ground to build on". Hence, despite the difficulties, the understanding of consciousness is still sought after.

In sum, consciousness is a vague and abstract notion which has fueled debates and discussions already for several centuries. Although various scholars have tried to define the notion, consciousness seems to be successful in avoiding denotation. Curiously, the rise of behavioral psychology did not only affect the attention to consciousness (Flanagan 1995), but, as will be mentioned in Chapter 4, it also was partly responsible in the marginalization of Institutional Economics from academic circles. However, even if various people have placed efforts in quining the term (and its study has been unpopular at some points in history), it continues to have an important stage in scientific research where its understanding has been approached from different paths. Finally, and perhaps more importantly, given that practical consciousness is particularly important for structuration theory; it will be interesting to analyze how the results of the conceptual and theorization exercise on cost consciousness are related to the tacit and nondiscursive skills that reflect practical consciousness. It is possible that the difficulty in defining cost consciousness is, at least partially, a result of cost consciousness reflecting more practical than discursive features.

\section{On Costs and Management Accounting and Control Systems}

The modifier of the cost consciousness compound is cost and, as mentioned in the introduction, management accounting systems (MASs) are intricately related to costs. Moreover MASs have drawn a considerable amount of attention form management accounting scholars. Hence, as this subchapter is reviewing some concepts, it seems a proper place to provide a brief overview on some aspects relevant to M ASs: definitions, types, examples, drivers, and results.

Chenhall (2003) states that management accounting (i.e. the collection of practices such as budgeting or product costing); MASs (i.e. the systematic use of management accounting to achieve some goal); management control systems (i.e. a broader term that encompasses M ASs); and organizational control (i.e. controls used to refer to controls built into activities and processes such as statistical quality control) are notions which are sometimes used interchangeably. Complicating matters further (at least conceptually), Tsui (2001) argues that accounting information systems (AIS) can be considered as control tools which include MAS information. 


\section{On M anagement Control Systems}

As management control systems (MCSs) encompass MASs, it might be wise to provide a brief overlook into these. According to Fisher (1998 p. 26) the ambiguous and contradiction in defining a control system is one of the difficulties in discussing management control systems (MCSs). Fisher (1995, pp. 25-26) presents the following definitions of control: (i) an involvement of subordinates in their activities by applying "such means as programming and standard operating procedures"; and (ii) cybernetic control referring to "a system in which standards of performance are determined, measuring systems gauge performance, comparisons are made between the standards and actual performance, and feedback provides information on the variances". Furthermore, Falkenberg and Herremans (1995 p. 134) differentiate between formal and informal (behavioral) control systems. While formal systems are "identified as the written procedures and policies that direct behavior so as to achieve the organization's goals, and/or detect/deter misconduct"; informal systems comprise the "common, values, beliefs, and traditions that direct the behavior of group members [who] learn the values, beliefs and traditions through a subtle reading of signals relayed by supervisors and co-workers".

Moreover, Tucker et al. (2009) state that MCSs are "the set of procedures and processes that managers use to help ensure that employees achieve both their own and their organization's objectives". Furthermore, the authors argue that although considerable amount of MCS literature draws on formal control systems, some approaches have pointed to an incorporation of both formal and informal systems in an attempt to present MCSs as a package. Within this respect, Malmi and Brown (2008) provide a conceptual typology of M CSs as a package which includes five types of control in the typology: planning, cybernetic, reward and compensation, administrative, and cultural controls. First, planning (e.g. action and long-range planning) refers to the ex-ante form of control as it sets out the goals of the functional areas of the organization; provides standards to be achieved in relation to the goal; and enables congruence by aligning goals across the organization's functional areas. Second, cybernetic control (e.g. budgets, financial and non-financial measures) includes measures to enable quantification of an underlying phenomenon; standards of performance/targets to be met; a feedback process that enables comparison of the outcome of the activities against the standard; and an ability to modify the system's behaviour or underlying activities. Third, rewards and compensation systems refer to the motivation and increase of performance of individuals and groups through rewards. Fourth, administration controls (e.g. organisational design and structure, procedures and policies administrative systems) refers to direct behaviour through organizing individuals; monitoring of behaviour; and specifying how tasks or behaviours are to be performed. Finally, fifth, culture (e.g. value-based control, symbols) relates to the established values, beliefs and social norms influencing employees' behaviour.

\section{On Management Accounting Systems and Practices}

Similarly with respect to MCSs, the concept of MASs seems to overlap other notions such as management accounting practices. For example, Busco et al. (2007) state that "management accounting practices can be interpreted as 'objects' that can extend and mobilize networks of relations with users and other organizational information systems to construct organization-wide visibility". The use of the word other clearly implies that, for the authors, management accounting systems and practices are synonyms. Hence, in this dissertation, these two notions are considered as synonyms. 
Furthermore, the literature on MASs has also focused on classifications and analysis of results associated to the notion. Chenhall and Langfield-Smith (1998a) have categorized management accounting practices into two main groups: (a) traditional techniques (e.g. the use of budgeting systems for planning and control, performance measures such as ROI, divisional profit reports, and cost-profit-volume techniques for decisions) and (b) contemporary practices (e.g. various forms of benchmarking, activity-based techniques, such as activity-based costing and activitybased management; balanced performance measures; team-based performance measures, employee-based measures; and strategic planning). Furthermore, the authors provide a list of examples of management accounting practices including ${ }^{12}$ : long term planning tools (e.g. capital budgeting techniques), budgeting systems (e.g. aiming at controlling costs), product costing systems (e.g. absorption costing, Activity-Based Costing), performance evaluation tools (e.g. budget variance analysis), and decision support systems (e.g. cost-volume-profit analysis). These examples, furthermore, highlight the intricate relationship between costs and management accounting systems. In addition, these examples also suggest a conceptual overlap between management accounting practices and MASs, as other authors also consider, for example, Activity Based Costing (ABC) as a MASs (see examples in Hussain et al. 1998 ${ }^{13}$, and Tsui 2001). Furthermore, expanding the conceptual overlap, Tillema (2005) also considers ABC as a management accounting technique and, furthermore, inter-exchanges the two terms (p. 102).

Moreover, Rasid et al. (2011) state that management accounting practices can achieve the following results: (i) identify the cost and profitability of doing business by organization, product, and major customer; (ii) avoid surprises; (iii) allow all managers to explain their performance as it is reported; (iv) permit everyone to participate in planning, and plan-to-actual reporting is used as a management tool; (v) provide timely, accurate, relevant, and comprehensive reporting; (vi) ensure that only one set of numbers is floating around the organization; and vii) reduce or eliminate complaints about information availability. These purposes are very similar to the ones suggested by Lea and M in (2000) (i.e. provide accurate product cost information, derive the most profitable product mix decisions, minimize inventory, and maximize profit and customer service under a given manufacturing control system). Hence, this provides another example of an overlap between MASs and management accounting practices. Furthermore, it has been noted that MASs have other purposes, for example they allow enhancing companies' ability to differentiate products and satisfy customers (Jermias and Gani 2004), to adopt and implement plans in response to their competitive environment, and to provide benchmarking, monitoring, and historical information (Mia and Clarke 1999).

In an attempt to maintain consistency, I will abstain myself from inter-exchanging MASs with management accounting practices and techniques. In addition, given the clear conceptual overlap, I consider the categories and examples of management accounting practices by Chenhall and Langfield-Smith (1998a) presented above as categories and examples of M ASs. However, this dissertation takes a very broad view on the concept of MASs following the conception by Macintosh and Scapens $(1990,1991)$ who consider that MASs have a role in the creation, regulation and transformation of an organization's social order by representing Anthony Giddens's "modalities of structuration in the three dimensions of signification, legitimation and domination" (1990, p. 462). It is important to note that Macintosh and Scapens draw from Roberts and Scapens (1985) who actually do not mention MASs but, instead, focus on accounting

\footnotetext{
${ }^{12}$ The complete list of management accounting practices which Chenhall and Langfield-Smith considered in their study can be observed in Appendix $C$.

${ }^{13}$ In the study by Hussain et al. (1998, p. 45), the concepts MASs and management accounting practices are actually integrated into M AS practices.
} 
systems. However, the conceptualization of Macintosh and Scapens allows the holistic perspective which this dissertation pursues and, perhaps more importantly, allows a convenient use of structuration and institutional theory in management accounting studies. This discussion will be expanded thoroughly in Chapter 4.

Finally, Granlund and Lukka (1998) identify four drivers of management accounting practices and categorize them between those which drive convergence and those which drive divergence. The four drivers are economic pressures underlying a considerable part of the global trend of management accounting practice; coercive pressures reflecting the enforcing regulative aspects of certain institutions; normative pressures concerning social obligations and appropriate social conduct in human behaviour; and mimetic processes which are linked to the cognitive and socially constructed side of human behaviour. Economic pressures include global economic fluctuations, increased competition, and advanced production and information technologies which are all factors driving convergence. Moreover, coercive factors driving convergence include transnational legislation and trade agreements, harmonization of the financial accounting legislations, and transnational's influence on subsidiaries. Coercive pressures driving divergence include national legislations, national institutions and regulations. Furthermore, while normative pressures driving convergence include management accountants' professionalization and university research and teaching; those driving divergence include national and corporate cultures. Finally, mimetic processes always drive convergence and include imitation of leading companies' practice and the international/global consultancy industry.

In sum, this section has explored the MASs notion and its conceptual overlap with management accounting practices and management accounting techniques. Moreover, it has been stated that this dissertation will take a very broad view of MASs in line with the conception by Macintosh and Scapens (1990). Furthermore, some purposes and drivers of MASs were also identified. Examples of management accounting practices cover a wide range of options including performance evaluations based on employee attitudes, capital budgeting techniques and detailed budgeting systems for compensating managers. These practices, in addition, are intricately related to costs, which serves as cost consciousness's linguistic modifier. M oreover, in discussing the drivers of management accounting systems, Granlund and Lukka (1998) identified corporate cultures as examples of normative pressures driving divergence. Hence, if cost consciousness could connote a type of corporate culture, then it could be capable of serving as a normative pressure driving divergence of management accounting practices. Granlund and Lukka argue that as corporate cultures set norms on organizational behaviour, they will maintain their influence regarding management accounting practices. However, the authors state, that the driving of divergence in management accounting practices by corporate culture is predominantly at the micro level given that, on the macro level, companies seem to be persuaded to similar or corresponding management accounting ideas irrespective of their possibly differing corporate cultures.

Finally, this subchapter has revised the linguistic composition of cost consciousness which can be reasonably considered as an endocentric compound connoting a consciousness on costs. By reviewing the linguistic head of the compound (i.e. consciousness) it was noted that the difficulty in providing a discourse and explanation on what cost consciousness is, might be a result of cost consciousness reflecting practical consciousness. This, moreover, is certainly welcomed in this dissertation as practical consciousness is very relevant for structuration and institutional theories. Moreover, instead of focusing on the linguistic modifier of the notion (i.e. cost) per se, I reviewed MASs which take a central role in the use of institutional theory in the management 
accounting studies. However, as stated in the beginning of this chapter, only by conceptually developing the notion can it be possible to assess what cost consciousness may hold. Hence, although cost consciousness seems to evade definition, a literature review on the possible drivers, interpretations, and results associated with the notion might provide a foundation for developing our understanding of cost consciousness. Moreover, this literature review should reveal the role of management accounting practices with respect to cost consciousness.

\subsection{Cost Consciousness: Use, Determinants, Results, and Interpretations}

Cost consciousness's linguistic composition suggests potential for research in the fields of accounting and management accounting. However, in an attempt to further our understanding of cost consciousness, and start uncovering what cost consciousness holds, a literature review has been conducted with two main objectives. First, the literature review provides a sense of the use of the notion not only in management accounting literature, but also across the academic disciplines. Second, the literature review aims to uncover the definitions, interpretations, drivers (i.e. antecedents), and results (i.e. consequences) which management accounting scholars have associated with the notion in an attempt to start constructing the foundations which may allow a further conceptual development of the notion.

\section{A sense of the use of cost consciousness}

In an attempt to take stock on the use of cost consciousness, not only in the field of management accounting but also throughout the academic spectrum, a cross-disciplined computerized review was conducted on November 2010. This review used five different scientific journal databases, namely EBSCOhost, Elsevier Science Direct, JSTOR, Emerald and ABI-inform. Although some authors have used one database as part of their literature review (see Rom and Rohde, 2007), using more than two databases reduces the probability of the literature background to be either narrow or shallow (Levy and Ellis 2006). Furthermore, in fact, two searches were conducted and their results can be observed in Table 1. This first search included two different methodologies which produced two sets of results. The first set of results was obtained by inserting the phrases 'cost consciousness' and 'cost conscious' as search-phrases and using the default search settings of the five databases. This basic search provides a valid starting point as the search algorithms that search engines use to retrieve documents have improved in recent years (Rowley and Slack, 2004). M oreover, the second set of results was obtained by limiting the search results to articles which included either 'cost consciousness' or 'cost conscious' in their titles, abstracts or keyword. The objective of this second search was to identify those articles where cost consciousness is not merely mentioned, but rather holds a more central role.

The results which appear in Table 1 show that searching for cost consciousness in major scientific literature databases can result in the identification of hundreds or even thousands of articles. Moreover, while the oldest article included in the first set of results - using default search settings - was authored by Forster (1932) in the Social Forces journal; the oldest accounting related publication, in the same results set, was authored by Taggart and Castenholz (1935) and it was published in The Accounting Review. 
Table 1. Search results for "cost consciousness" or "cost conscious" in different databases

Scientific Journal Databases

\begin{tabular}{|c|c|c|c|c|c|}
\cline { 2 - 6 } \multicolumn{1}{c|}{} & EBSCOhost & Elsevier SD & JSTOR & Emerald & ABI inform \\
\hline $\begin{array}{c}\text { Default Search } \\
\mathrm{N}=4963\end{array}$ & 191 & 1928 & 2031 & 658 & 155 \\
\hline \\
\hline \\
\hline
\end{tabular}

Furthermore, the second set of results identifying 514 articles includes 76 duplicates; therefore, the sample is reduced to 438 articles belonging to 311 different Journals (Appendix A). In addition, Appendix $A$ also provides evidence of the use of the cost consciousness notion across different fields of study including accounting, healthcare and medicine, education, computer sciences, biology, economy, finance, operations management, business, technology management, sociology and law.

Hence, cost consciousness seems to be used across academic literature. Furthermore, the results of this literature review suggest that the notion is more common in health care related disciplines when compared to management accounting. Therefore, the discussions on the interpretations of cost consciousness from a management accounting perspective could find support from the interpretations from the health care perspective.

\section{Cost Consciousness's use in Management Accounting Literature}

Although cost consciousness is used across various academic disciplines, this study focalizes in the field of management accounting. Therefore, it is important to review the use of the notion in management accounting related journals. The list of 39 relevant journals (Appendix B) was obtained from the literature review conducted by Rom and Rohde (2007).

Moreover, the five databases which were used for the cross-discipline search were also used to search for the search-phrases 'cost consciousness' or 'cost conscious' in these thirty-nine relevant journals, resulting in the identification of 190 peered-review articles which mention the search-phrases at least once in their text.

However, from these, I could not access 8 due to restrictions set by the journals and databases. Hence, the sample is reduced to 182 articles. Moreover, 11 of these 182 articles included the words cost consciousness or cost conscious only in their references or as very loose terms in tables and appendixes referencing to other works. As these 11 articles did not provide the opportunity to identify or infer how the authors interpreted the notion, I decided not to take these into account. Therefore, the original sample of 190 articles was ultimately reduced to 171. The largest amounts of scrutinized articles were found in the journals Accounting Organizations and Society and Management Accounting Research where 19 articles were identified in each. Moreover, 16 articles were found in The Accounting Review, 15 in Journal of Management Studies, 10 in Administrative Science Quarterly, and 9 in Academy of M anagement Journal. These

\footnotetext{
${ }^{14}$ The results suggest that the default search settings of EBSCO and ABI are set to identify the searchphrase(s) in the title, abstract or keyword of the articles.
} 
88 articles represent more than half of the sample. Hence, these results provide evidence of the use of the cost consciousness notion in most management accounting relevant journals.

However, only two definitions of cost consciousness (Kurunmäki 1999; and Nor-Aziah and Scapens 2007) were rescued in the 171 scrutinized articles, and, furthermore, only two studies (Shields and Young 1994, Abernethy and Vagnoni 2004) studied possible drivers of cost consciousness. Therefore, this study calls for developing the understanding of cost consciousness not only because of the obligation of research in enhancing the scientific community's current understanding of a phenomenon (Levy and Ellis 2006), but also due to the low number of studies which thoroughly define or analyze the notion despite the fact that scholars have been mentioning it for many years and across diverse disciplines.

Hence, as a literature review is not only an essential feature of any academic project, but it also creates a firm foundation for advancing knowledge, facilitating theory development, closing areas where a plethora of research exists, and uncovering areas where research is needed (Webster and Watson 2002); the following sections identify and discuss the interpretations, drivers, and results which were rescued from the 171 articles found in the relevant management accounting journals.

\section{Definitions and Interpretations of Cost Consciousness}

This section discusses the definitions and interpretation that management accounting scholars have associated with of cost consciousness.

Kurunmäki's definition: Economic judgement of health care operations

From the 171 scrutinized publications, the earliest peered-reviewed article providing a definition of cost consciousness is authored by Kurunmäki $(1999$, p. 121) who states:

An increased awareness of costs, facilitated by the new and more detailed cost accounting systems, was evident to all health professionals interviewed at both university and central hospitals. Further, the improved cost consciousness - the economic judgement of health care operations - was regarded as having implications for the clinical decision making processes

One could reasonably argue that the words inside the hyphens (i.e. economic judgment of operations) denote cost consciousness in the previous quote.

However, this definition of cost consciousness forces one to ponder about what economic judgement connotes. According to the Oxford Dictionary of English (2006), judgement may be defined as "the ability to make considered decisions or come to sensible conclusions". Hence, the definition by Kurunmäki suggests that cost consciousness connotes the ability to make considered decisions or come to sensible conclusions with respect to economic issues in operations. The understanding of this extended definition, however, would necessitate a consensus on what considered decisions and sensible conclusions may connote. While consider is defined as "think carefully about (something), typically before making decisions", sensible is denoted as "done or chosen with wisdom and prudence". Therefore, cost consciousness may be extended to denote the ability to think carefully about economic operations with wisdom and prudence. However, once again we arrive to a definition which forces one to ponder about what 
thinking carefully, and wisdom and prudence may connote. Hence, the dictionary shows once again its clear limitations with respect to the clarification of notions. However, this type of analysis may very well result in the same type of conceptual dilemma in any notion; hence one could reasonably argue that the original definition by Kurunmäki is relatively clear as it at least allows the identification of a definition of the construct.

Furthermore, Kurunmäki invokes cost consciousness in other occasions in her study:

During the late 1980s and early 1990s, attempts to increase the cost-consciousness of health professionals and improve the ability of health care financiers to control the service providers resulted in hospitals being encouraged or pressurized to introduce substantial modifications in their official accounting practices. This included changes in budgeting, cost accounting and financial reporting. It is to these changes in practice that we now turn. (p. 103)

The impact of enhanced knowledge of costs on operational choices in one university hospital service unit was expressed by the chief physician of this unit as follows: "A good indication that we have continuously made calculations and debated our operations can be seen in our article that will be published in the American Journal of $X$, which considers the examination methods of $Y$; which is the most economical way. .. So these things should all the time be considered also from that (economic) point of view, not only from the medical viewpoint. . . and, yes, this is what we have tried to do[... I I think, as far as the attitudes of the staff is concerned, that the impact of the whole costing project has furthered it (cost consciousness) enormously. One knows exactly whether it is reasonable to do this or that, or whether we should use that one, since it's more economical." Yet despite the widespread perception that the cost consciousness of health care professionals had increased substantially, hospital representatives often found it difficult to isolate either the impacts of Results M anagement programs, or the effects of an attempt to set up market forces in health care, from the wider social pressures for increased cost efficiency in the public sector. (p. 121)

The quote on page 103 does not provide any real definition of cost consciousness, suggesting that the author assumes that the reader shares her tacit interpretation of the notion. However, the first quote does allow one to infer that by modifying accounting practices, cost consciousness may be increased. Hence, although no clear interpretation can be inferred, the author suggests a management accounting practice as a driver of cost consciousness. M oreover, with regard to the quote in page 121, the author infers cost consciousness from the answer of the chief physician. However, in this case, cost consciousness does not exactly refer to economic judgement of health care operations, but seems to refer to enhanced knowledge of costs on operational choices. This interpretation is somewhat clearer as one can reasonably argue that knowledge of costs is easier to interpret when compared to economic judgement.

Nor-Aziah and Scapen's definition: Economic efficiency

The second definition of cost consciousness identified in the scrutinized management accounting journals is provided by Nor-Aziah and Scapens (2007, p. 217):

PSP is an established, large, geographically dispersed and labour-intensive organisation which provides services to the public [...] Furthermore, there are 
institutional pressures of both a coercive and a regulative nature which have continued to come from the government. The government requires PSP to be economically efficient, i.e. a cost-conscious organisation, but at the same time prevents it from behaving in a strictly commercial manner.

One can reasonably argue that cost consciousness is defined as economical efficiency in the previous quote. This definition, moreover, similarly to the one by Kurunmäki (1999) is arguably very holistic in nature.

Moreover, Nor-Aziah and Scapens (2007) invoke the notion in two other occasions in their work:

To encourage cost-conscious behaviour, the State accountants were expected to report cost variances to both their State managers and the operations accountant (in the Performance Analysis Unit) at HQ on a monthly basis. The new reporting process was intended to enable the $\mathrm{HQ}$ managers to monitor the spending behaviour of the operations managers at the State offices. This monitoring from a distance was deemed essential to increase visibility and control over both the financial and operational aspects of the State offices' performance. (p. 222)

In PSP, the new accounting (budgetary control) system was intended to improve the cost-consciousness of the managers responsible for the provision of public services. But in seeking to promote financially oriented behaviour, the accounting controls reduced the autonomy of the operations managers and gave them the impression that they were not to be trusted; they, in turn, reciprocated by not trusting the accountants or their budgeting systems. (p. 231)

The first quote in page 222 allows one to infer that cost consciousness is interpreted as some sort of desired behaviour which could be driven by the a periodical monitoring involving a new reporting process. Moreover, the quote on page 231 suggests a relationship between cost consciousness and a reduced autonomy which affected trust. However, it is difficult to state if that negative result is produced by cost consciousness or by the means in driving cost consciousness. Hence, the difficulties in conducting this conceptual exercise are highlighted in this particular example.

As the reader could note, neither Kurunmäki or Nor-Aziah and Scapens provided definitions of cost consciousness by using a direct mode such as "cost consciousness can be defined as..." or "cost consciousness represents a..." Hence, I would like to present one definition of cost consciousness which does follow a "cost consciousness is defined as..." mode. However, it is important to note that this definition appears in a health care related publication. Nevertheless, given the very common use of the notion in health care publications, I believe that it can provide support for the conceptual development of the notion 
Bovier et al. providing a glimpse of the health care interpretation

The following quote is found in the BMC Health Services Research Journal in the article by Bovier et al. $(2005$, p. 2$)$ :

In this paper, we are interested in cost-consciousness, defined as a concern to contain costs of health care borne by society [Greene et al. 1989] ${ }^{15}$

There are two important issues which rise from this definition. First, if one extends a concern to contain costs of health care borne by society, in a similar way as previously conducted, one can extend the definition of cost consciousness to a concern to control or restrain costs. Therefore, this definition is clearer and conceptually narrower when compared to the definitions discussed previously given that contain provides a seemingly narrower conceptual realm when compared to words like judgement (in Kurunmäki's definition) and efficiency (appearing in the denotation by Nor-Aziah and Scapens). The second issue is, interestingly, that Greene et al. (1989) did not mention the cost consciousness notion in their work. Furthermore, after reviewing the study by Greene et al. one can reasonably argue that Bovier et al. assumed that it was possible to interexchange cost consciousness with cost containment, the term which actually appears throughout the work by Greene et al., even in its title - 'Physician Attitudes Toward Cost Containment'.

Moreover, Bovier et al. (2005), perhaps inadvertently, provide another possible definition of cost consciousness in their article through a translation into French which they propose for the following sentence:

It is unfair to ask physicians to be cost conscious and still keep the welfare of their patients foremost in their minds (p. 2)

The following translation into French was presented by the authors:

Il est injuste de demander aux médecins de penser á réduire les coûts de la santé, tout en se préoccupant avant tout du bien-être de leurs patients. (p. 2)

Therefore, one can deduce that cost consciousness was translated into penser á réduire les coûts which translates back to English into consider reducing the costs. Hence, as reducing (i.e. lowering) is not necessarily the same as containing (i.e. control, restrain, prevent expansion), then a subtle but, nevertheless, considerable inconsistency arises.

Hence, the review of this definition in a health care related journal, although at first glance provides a clarification on cost consciousness, a closer look suggests inconsistency, therefore conceptual opacity.

\section{Discussion on the Definitions of Cost Consciousness}

A couple of interesting issues can be addressed after reviewing the definitions and interpretations of cost consciousness by Kurunmäki (1999) and Nor-Aziah and Scapens (2007). First, none of the authors presented their definitions of the notion by using phrases such as "cost consciousness is ..." or "cost consciousness is defined as ...", but rather opted for the use of other means such as a hyphen in the case of Kurunmäki and the use of id est. in the case of Nor-Aziah and Scapens. This suggests the possibility of an intentional avoidance in defining the construct.

\footnotetext{
${ }^{15}$ In the original article, a number identifying the note/ reference appears inside the brackets.
} 
Second, both definitions are abstract even if one tries to extend them by taking a deductive approach. Moreover, this approach highlighted the clear limitations of a dictionary. Third, both definitions include the economic adjective which, in itself, is arguably broad and involves a vast conceptual realm. Therefore, one could reasonably argue that cost consciousness, if conceptually developed, could also serve in the field of economics. Fourth, the reviews on the additional interpretations of cost consciousness allowed highlighting some associated results and drivers of the notion. Moreover, with respect to the drivers, MASs seem to play a role as antecedents of cost consciousness. Fifth, acknowledging that the existence of supra-individual knowledge has been considered for some time resulting in the research of knowledge structures not only at the individual, but also at the group, organization and industrial level of analysis (Walsh, 1995); there is reason to believe that a similar classification could be used for studying cost consciousness. For example, Kurunmäki (1999) - and also Bovier et al. (2005) - suggest that the notion is a characteristic of physicians and health care professionals respectively, thus supporting the existence of cost consciousness at the individual level. Moreover, Nor-Aziah and Scapens (2007) suggest that cost consciousness is a characteristic of the organization as a whole, thus providing support for considering cost consciousness a characteristic at the organization level. Sixth, while the definitions by Kurunmäki (1999) and Nor-Aziah and Scapens (2007) suggest a very broad conceptual realm, the inconsistent definitions provided by Bovier et al. (2005) are conceptually narrower as they refer to capabilities or actions with a specific goal, in this case, containing and lowering costs.

In sum, this section has discussed the two definitions of cost consciousness identified from the one-hundred-and-seventy-one scrutinized articles published in management accounting related journals. Therefore, the following subsection reviews some of the interpretations which can be rescued from the other 169 articles aiming to extend the understanding of how cost consciousness is interpreted by management accounting scholars.

\section{Inferred Interpretations of Cost Consciousness}

This subsection scrutinizes articles where cost consciousness is mentioned, but not defined, in an attempt to shed light on the interpretations of cost consciousness.

I would like to begin this exercise with the following quotes:

The basic thinking here is that contractors with high risk contracts would tend to good management and be motivated to cost consciousness. These factors [...] would accomplish more to effective control of costs than the detailed administrative controls and audits by the government. (Trueger 1967, p. 49)

As a result, reform advocates claim agencies faced with market pressures and requirements to recover all expenditures with revenues will become more business like and cost conscious, and will make greater use of cost accounting data in order to control costs and prevent funding shortfalls and associated sanctions (Geiger and Ittner, 1996, p. 551)

In their strategies to change practice patterns and heighten cost consciousness, the clinic managements emphasized the participation of staff (through discussions at staff meetings) in decisions concerning changes in work procedures. The managements sought to encourage cost containment by advising staff on ways of improving medical care and on cheaper treatment alternatives rather than by 
restricting the prescription of drugs and medical treatments (p. 173) [...] In addition, [the program] includes strong incentives for physicians to change their practice patterns, heightening their cost consciousness and promoting cost containment ( $p$. 174). (Gross et al. 1996)

The quote by Trueger (1967) suggests a very close relationship to cost control (i.e. containment). Hence, one could reasonably argue that Trueger's interpretation of cost consciousness is similar to one of the conceptually narrower definitions uncovered from the study by Bovier et al. (2005).

Furthermore, the reference by Geiger and Ittner (1996) suggests cost consciousness to be a characteristic of agencies (i.e. cost consciousness at the collective level). In addition, the authors also seem to interpret cost consciousness as cost control (i.e. containment). Moreover, this quote allows one to infer market pressures as a possible driver of cost consciousness. Within this respect, I believe that it is difficult to determine if a better use of accounting data acts as a possible driver or result of cost consciousness.

In addition, the quotes by Gross et al. (1996) raise a couple of issues. From the quote on page 173, cost consciousness seems to refer to cost containment. However, the quote on page 174 seems to suggest not only that cost consciousness and cost containment are different concepts, but that cost consciousness drives cost containment. With respect to this problem, Bem (1995) states that in order to achieve accuracy - which is paramount in technical writing - and clarity, scholars should opt for repetition and parallel construction in order to avoid readers to justifiably wonder if different meanings are implied whenever authors use different words to refer to the same concept. Finally, Gross et al. also seem to associate staff participation in decision concerning changes in work procedures as a driver of cost consciousness.

Therefore, the first set of quotes presented in this section suggest that, although the definitions found in management accounting journals reflected very broad conceptual realms, some management accounting scholars seem to interpret cost consciousness in a more narrow sense.

Apart from cost containment, the quote by Bovier et al. (2005) allowed the identification of cost reduction as another possible interpretation of cost consciousness. With respect to the latter, the following quotes from management accounting journals provide support:

[The increased emphasis attached to costs and cost reductions] also served to promote cost consciousness as a mobilizing organizational imperative. Being good at achieving cost reductions became an organizational objective that competed with the predominantly engineering judgments about standards of service that had previously prevailed. (Ogden 1995a, p. 198)

Eldenburg (1994) empirically examined whether more frequent reporting of cost information to hospital physicians could induce cost-conscious behavior. However, contrary to her hypothesis, higher reporting frequency was not significantly associated with lower costs. (Pizzini 2006, p. 184)

As the reader may note, both quotes seem to suggest cost consciousness to connote cost reduction. M oreover, while the quote by Ogden (1995a) seems to consider cost consciousness as a collective characteristic, the statement by Pizzini (2006) suggests cost consciousness to be inherent to an individual. Furthermore, the quote by Ogden suggests that emphasizing costs and cost reduction leads to cost consciousness. Hence cost consciousness is a result in itself. 
Furthermore Pizzini's quote suggests that higher reporting frequency is not a driver of cost consciousness.

This subsection, therefore, has served to provide evidence of management accounting related interpretations of cost consciousness which seem to suggest a narrow conceptual realm contrasting the more holistic definitions by Kurunmäki and Nor-Aziah and Scapens (2007). However, given the linguistic compositions of cost consciousness, one could reasonably expect a holistic notion to be shared by various authors. Therefore, in expanding this analysis I invoke the following quotes:

In the central administration, it was perceived that NordDRG-based financial and clinical information had contributed to increased cost consciousness [...] Increased cost consciousness, in turn, was seen to have had a positive impact on the efficiency of profit centers, as it promoted rationing of clinical activities (p. 380) [...] it was felt that NordDRG-based prospective pricing and case-mix accounting systems had increased cost consciousness (p. 381) [...]In addition, health care professionals appear to have become cost-conscious and increasingly aware of their limited financial resources (pp. 390-391) (Lehtonen 2007)

The key distinction is between local and global cost consciousness. A local focus occurs in a single unit of an $R \& D$ organization or it may be the $R \& D$ unit as a whole. A global focus is one in which the professional considers the total costs to the organization of decisions. For an R\&D professional, a global focus means including all downstream costs to $R \& D$ as well as all $R \& D$ costs (Shields and Young 1994, p. 181)

Both quotes seem to suggest that cost consciousness represents a type of general awareness; hence, a more holistic conceptual realm when compared to the other sets of quotes reviewed in this subsection. M oreover, while Lehtonen's quotes clearly characterize cost consciousness at an individual level; the quote by Shields and Young (1994) differentiates the collective level between cost consciousness in an organization, and cost consciousness in a single unit. Furthermore, Lehtonen's quotes not only suggest that cost consciousness results in efficiency of profit centers and identification of limited financial resources, but also highlights the role of MASs in driving cost consciousness.

\section{Cost Consciousness and Organizational Culture}

The introduction of this dissertation presented the quote by Dekker (2004) which suggested that cost consciousness could be considered as an organizational culture. This connotation certainly suggests a holistic conceptual realm similar to some of the quotes presented in the previous subsection. This subsection, moreover, presents the following quotes which suggest a very close relationship between cost consciousness and organizational culture:

During the regional 'soapbox' meetings the main core values that promote a business mind were: learning to work efficiently, stimulating a commercial attitude among salespersons and teaching physicians as fee earners to become more cost conscious in the post-MBO situation. The CEO has very clear view on the need for employees to adopt the new beliefs and culture (Bruining et al. 2004, p. 163) 
Moreover, Southwest uses task to support the culture underlying treatment. The company has a formal committee tasked with keeping the companies "small family and spirit" (Hallowell and Heskett 1993). Careful attention is paid to the training process, "Not only are the values of hard work, fun, and cost consciousness inculcated, but the training is used to get internal customer feedback" to see what needs to be done to keep the culture alive (Stewart 2003, p. 258)

Both quotes seem to suggest that cost consciousness may represents a belief associated with a particular organizational culture. Furthermore, as chapter 4 will explain, if these beliefs would be successfully repeated enough, they might become taken-for-granted and transform themselves into an organizational culture. Moreover, Smith et al. (1981, p. 403) suggests that cost consciousness could represent an organization's philosophy, a concept which is closely related (from an intuitive point of view) to organizational beliefs and values.

Furthermore, the following set of quotes provides associations between cost consciousness and organizational culture which are even more explicit:

However, the culture is cost conscious and despite the amount of international travel Taylor and his employees undertakes (Taylor frequently travelled outside Australia three weeks out of four), he sets an example by continuing to travel economy class. (Collier 2005, p. 332)

More important than specific incentive plans is the establishment of a cost conscious managerial orientation among the systems specialists. The existence of such a culture among specialists who identify with a profession depends on its existence in the organization in general and also the extent to which such an orientation is consistent with the values of the professional establishment outside the organization. (Young 1981, p. 32)

Other examples of explicit associations between cost consciousness and organizational culture can be found in Baird et al. (2004, p. 388), Ogden (1995b, pp. 23-24), and Seal and Croft (1997, p. 76).

Summarizing, this section has presented and discussed two definitions and various interpretations of cost consciousness from articles published in management accounting journals. Moreover, while some definitions and interpretations suggest a narrow conceptual realm (e.g. as cost reduction or cost containment), others are holistic nature. Furthermore, some of the conceptually broader interpretations suggest that cost consciousness is a type, or an important feature, of organizational culture. M oreover, while some interpretations of the notion characterize an individual, others describe a department/unit or an organization.

Table $\mathbf{2}$ provides a summary of how the interpretations of the reviewed studies could be placed with respect to degree of holism and the type of entity (i.e. individual or collective) which they characterize. It is important to note that an unclear conceptual broadness does not indicate that cost consciousness is used in an incomprehensive manner in a particular reference, rather that it is difficult to assert if the notion connoted a narrow or broad interpretation. 
Table 2. Summary of definitions and Interpretations of cost consciousness

\begin{tabular}{|c|c|c|c|}
\hline & \multicolumn{3}{|c|}{ Conceptual Broadness } \\
\hline & Holistic & Narrow & Unclear \\
\hline 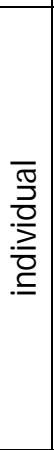 & $\begin{array}{l}\text { Thompson and Bates (1957, p. 336) } \\
\text { Smith (1980, p. 210) } \\
\text { Smith et al. (1981, pp. 399) } \\
\text { Llewellyn (1998, p. 26) } \\
\text { Stajkovic and Luthans (2001, p. 588) } \\
\text { Jones and M ellett (2007, p. 109) } \\
\text { Fleischman et al. }(2008, \text { pp. } 355,360) \\
\text { Gates and Langevin }\left(2010, \text { p. } 124^{16}\right)\end{array}$ & $\begin{array}{c}\text { Kohler }(1945, \text { p. 41) } \\
\text { Boutell }(1964, \text { p.306) } \\
\text { Smith et al. }(1981, \text { pp. 400, 403) } \\
\text { Young }(1981, \text { p. 30) } \\
\text { Tractinsky and Jarvenpaa }(1995, \text { p. 521) } \\
\text { Clemons and Hann }(1999, \text { p. 15) } \\
\text { West and Dedrick }(2000, \text { p. 208) } \\
\text { Siggelkow }(2002, \text { p. 147) } \\
\text { Sum et al. }(2004, \text { p. 339) } \\
\text { Tyrrall and Parker }(2005, \text { p. 526) } \\
\text { Mayer and Salomon }(2006, \text { p. 950) } \\
\text { Rindova et al. }(2006, \text { p. 60) } \\
\text { Kober et al. }(2007, \text { p. 442) }\end{array}$ & $\begin{array}{l}\text { Strauss }(1962, \text { p. } 179)^{18} \\
\text { Caplan }(1968, \text { p. 348) } \\
\text { Wood }(1996, \text { p. } 57) \\
\text { Douglas }(2003, \text { p. } 258)^{20}\end{array}$ \\
\hline 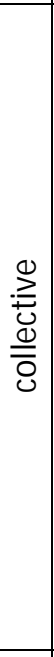 & $\begin{array}{c}\text { Cangelosi (1965, pp. 136, 138) } \\
\text { Khandwalla }(1973, \text { p. 293) } \\
\text { Dimick and M urray }(1978, \text { p. 621) } \\
\text { Young }(1981, \text { p. 32) } \\
\text { Nutt }(1983, \text { pp. 604,611) } \\
\text { Finlay }(1987, \text { p.57) } \\
\text { Murray and Reshef }(1988, \text { p. 620) } \\
\text { Whipp et al. }(1989, \text { p. 578) } \\
\text { Chan et al. }(2000, \text { p. 297) } \\
\text { Menon et al. }(2000, \text { p. 83) } \\
\text { Tam et al. }(2003, \text { p. 43) } \\
\text { Davila and Wouters (2004, p. 17) } \\
\text { Snyder et al. }(2005, \text { p. 87) } \\
\text { Zott and Huy }(2007, \text { p. 84) } \\
\text { Fleischman et al. }(2008, \text { pp. 358, 371) } \\
\text { Menon et al. }(2009, \text { p. 300) } \\
\text { Chenhall et al. }(2010, \text { p. } 749)\end{array}$ & $\begin{array}{c}\text { Taggart and Castenholz }(1935, \text { p. } 75) \\
\text { Fisher }(1970, \text { p. 367) } \\
\text { Provan }(1987, \text { p. 236) } \\
\text { Clemons and Row }(1991, \text { p.60) } \\
\text { Belcher and Watson }(1993, \text { p. 240) } \\
\text { Holden and Wilhelmij }(1995 / 1996, \text { p.31) } \\
\text { Tam (1996, p. 165) } \\
\text { Knights and M CCabe }(1997, \text { p. 376) } \\
\text { Smith et al. (1998, p. 66) } \\
\text { Clarke and Mia (1993, p. 81) } \\
\text { Choon and Wisner (2003, p. 1311) } \\
\text { Imhoff (2003, p. 119) } \\
\text { Owen (2003, p. 294) } \\
\text { Weiss et al. (2006, p. 685) } \\
\text { Maguire and Phillips (2008, p. 389) } \\
\text { Saravanamuthu (2008, p. 166) }\end{array}$ & $\begin{array}{c}\text { Tannenbaum (1962, p. 254) } \\
\text { Hafner (1964, p. 980) } \\
\text { Smith (1967, p. 137) } \\
\text { Legge (1970, p. 6) } \\
\text { Knutson and Wichmann (1985, p. 393) } \\
\text { D'Aveni and Ilinitch }(1992, \text { p. 601) } \\
\text { Foster and Gupta (1994, p.72) } \\
\text { Koch and Cebula (1994, p. 683) } \\
\text { Rainer and Watson (1995, p. 90) } \\
\text { M aunders (1997, p. 70) } \\
\text { M cDermott et al. (1997, p. 77) } \\
\text { Hofstede (1998, p. 4) } \\
\text { Batt (2002, p. 595) } \\
\text { Feng and Baruch (2004, p. 5) } \\
\text { Stonebraker and Liao (2004, p. 1048) } \\
\text { Conrad (2005, pp.7, 8, 18) } \\
\text { Agbejule and Saarikoski (2006, p. 436) } \\
\text { Bahmanziari et al. (2009, p. 162) } \\
\text { Tallon (2010, p. 245) }\end{array}$ \\
\hline $\begin{array}{l}\frac{\grave{d}}{\mathscr{U}} \\
\frac{\mathrm{J}}{J}\end{array}$ & $\begin{array}{l}\text { Fleischman et al. (1995, p. 166) } \\
\text { Bacharach et al. (2000, p. 724) } \\
\text { Wickramasinghe and Mills (2002, p. 94) } \\
\text { Chua and Mahama (2007, p. 64) } \\
\text { Fleischman et al. (2008, p. 359) }\end{array}$ & $\begin{array}{c}\text { Woodward (1982, p. 189) } \\
\text { Miller (1987, p. 695) } \\
\text { Antonacopoulou (2001, p. 344) } \\
\text { M ouly and Sankaran (2004, p.1452) } \\
\text { Lindgren and Packendorff (2006, p. 851) }\end{array}$ & $\begin{array}{l}\text { Olson and Ives (1982, p.49) } \\
\text { Goodhue et al. (1988, p. 384) } \\
\text { Chang (1995, p. 383) } \\
\text { Babbar and Prasad (1998, p. 425) } \\
\text { Broadbent et al. (1999, p.164) }\end{array}$ \\
\hline
\end{tabular}

Moreover, in certain cases authors swiveled between holistic and narrow interpretations of the notion. For example, Smith et al. (1981, p. 399) present a model including one element or box named Manifestations of Cost Consciousness (Figure 6).

\footnotetext{
${ }^{16}$ In page 124 , the authors do not mention cost consciousness but they describe their results in a manner which seems to suggest that their interpretation of the notion reflects an ability to manage costs. They mention cost consciousness in various other pages (e.g. pp. 111, 122).

${ }^{17}$ Cost consciousness appears in a quote presenting the answer of an interviewee

${ }^{18}$ Cost consciousness appears in a quote presenting the answer of an interviewee

${ }^{19}$ Cost consciousness appears in a quote presenting the answer of an interviewee

${ }^{20}$ Cost consciousness appears in a quote presenting the answer of an interviewee

${ }^{21}$ Cost consciousness appears in a quote presenting the answer of an interviewee
} 


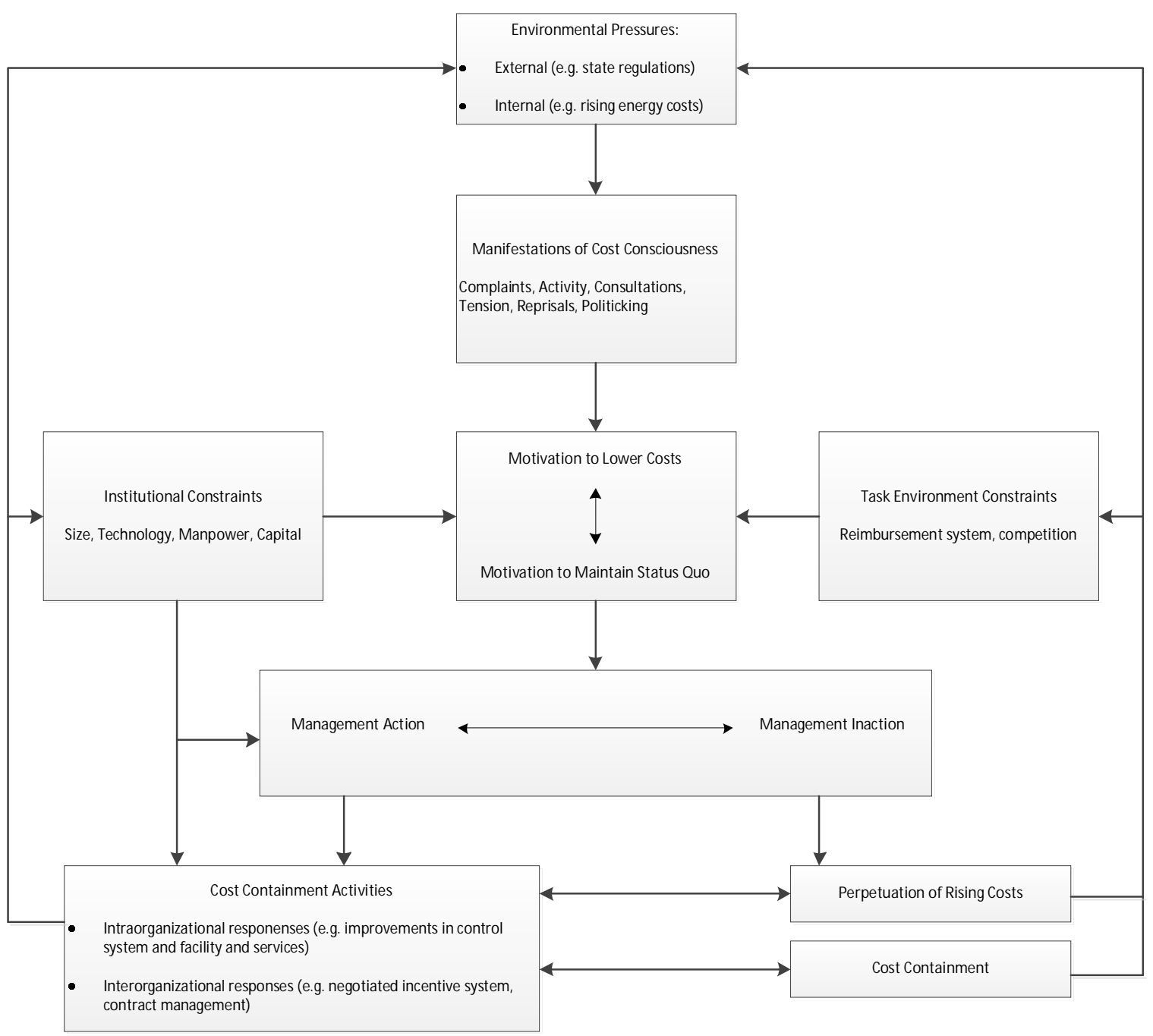

Figure 6. A view of Cost Containment in Health Care (adapted from Smith et al. 1981, p. 399)

As the reader can observe, different notions similar to cost consciousness, namely lowering costs and cost containment, appear in various boxes of the model displayed above. Although this would suggest a differentiation between these notions, the explanatory text in their study does not clarify these differences and, in certain parts, is quite confusing. For example, the authors write:

The model shows a number of different factors contributing to the cost consciousness of health care administrators [...] The external pressures help form the cognitive appreciation (or consciousness) by administrators of the need to contain costs. (Smith et al. 1981, p. 400)

Therefore, although it seems that Smith et al. differentiate between cost consciousness, lowering costs, and cost containment, the previous quote actually suggests that these notions are synonyms.

Finally, some of the quotes discussed in this section provide hints to drivers and results which management accounting scholars have associated with cost consciousness. With respect to Figure 6, for example, it would be possible to argue that environmental pressures act as drivers of the manifestations of cost consciousness. As understanding how to drive cost consciousness is a main topic of interest in this dissertation, the following section explores the possible drivers or determinants of cost consciousness. 


\section{The Determinants of Cost Consciousness}

This section focuses on identifying and inferring other possible determinants that management accounting scholars have associated with cost consciousness. Similarly to the exercise on the definitions and interpretations, this section will first address those studies that have evaluated drivers of cost consciousness before discussing the antecedents which can be inferred from the literature.

Determinants according to Shields and Young (1994) and Abernethy and Vagnoni (2004)

The article by Shields and Young (1994) studies the determinants of local cost consciousness where sixteen variables were hypothesized to affect the cost consciousness of $R \& D$ professionals. The authors explain:

This variable [cost consciousness] was measured by summing the responses to seven questions which asked about a respondent's general cost consciousness over a variety of projects during the last several years (see Appendix A). These questions asked about the levels of agreement or disagreement with seven items intended to assess whether the respondent and superior and co-workers were aware of costs, consider costs to be important, and take actions to be cost efficient Each question was responded to on a seven-point scale anchored by 1, "Strongly Disagree" and 7. "Strongly Agree." A higher value indicates a higher level of cost consciousness. (Shields and Young 1994, p. 184)

Moreover, for this evaluation, the authors asked respondents to evaluate the following statements (p. 192): (i) In general, I know what the overall level of the cost budget is for my unit; (ii) I have good knowledge of the way my unit's cost budget is spent; (iii) In general, I know what my co-workers spend on their projects; (iv) My boss goes over the cost budget with his or her employees so that they know the spending goals and limits; (v) As a group, my co-workers and I discuss the cost of projects on a regular basis; (vi) In general, when I am deciding whether to purchase some supplies or equipment, I focus heavily on how much it costs relative to the budget; and (vii) In general, when I am deciding whether to purchase some supplies or equipment, I focus heavily on how much it costs relative to the expected benefit to be derived from it.

Furthermore, the results from the Shields and Young study indicate that only three variables (i.e. cost management knowledge, cost budget participation and cost of regulation) out of a total of sixteen evaluated variables explained a significant amount of the variation in cost consciousness. Cost management knowledge, the authors argue, should not be a surprising determinant of cost consciousness as it would be difficult to understand how costs could be managed without knowledge about them. Furthermore, the authors argue that our understanding of how cost knowledge affects cost consciousness could be increased if we would, for example, identify the specify types of knowledge (e.g. activity-based costing, cost of time) which could have the greatest effect in cost management. Moreover, cost budget participation was found to have the largest effect on cost consciousness and, according to the authors, it is an effective and efficient means of facilitating the sharing of, and learning about, diverse and complex information by innovative workers. Finally, a significant inverse relationship between cost of regulations and cost consciousness resulted from the study by Shields and Young. The authors had expected that as regulatory costs increased (e.g. environmental, health, safety, legal, patent), R\&D employees 
would become more sensitive to costs. Furthermore, Shields and Young seemed to be surprised by the following four variables resulting in not being strong determinants of cost consciousness after the field interview suggested them to be potentially significant: (1) Scientific culture; (2) Cost based compensation; (3) Cost budget tightness; and (4) Top management attention to costs. Within this respect, the authors argue that these surprises could be due to problems relating to sampling, measurement, or modeling limitations. Finally, it is interesting to note that staff participation in decisions concerning changes in work procedures had been associated as a driver of cost consciousness in the quote of Gross et al. (1996) presented in the previous section. This associated driver, when compared to the finding by Shields and Young (i.e. cost budget participation as a driver of cost consciousness) suggest that employee participation in strategic decisions may act as a driver of cost consciousness.

Moreover, the study by Abernethy and Vagnoni (2004) assessed both the direct relation between authority structures (formal and informal) and cost consciousness as well as the indirect relations that occur via Accounting Information Systems (AISs). The authors explain (p. 210) that while formal delegation of decision rights is generally related to the incumbent's position in a ranked hierarchical structure; informal authority is defined as the ability of an individual or groups of individuals to influence organization decisions and activities in ways that are not sanctioned by the formal authority system.

In addition, Abernethy and Vagnoni used the instrument developed by Shields and Young (1994) to evaluate cost consciousness and their study provides three main results. First, the use of AISs has an effect on the cost consciousness of physicians by providing means of reinforcing the formal delegation of authority and complements the formal authority structure by articulating the value set associated with a commitment to resource management. Second, there is no direct relation between formal authority and cost consciousness; therefore, the delegation of authority to physicians influenced the use of AISs for control purposes but this did not influence the cost consciousness of physicians. Finally, the informal authority, which is not a significant antecedent to the use of AISs, was very significant in explaining the cost conscious behaviour of physicians. The authors argue that it would appear that the higher the level of power of physicians the less they are likely to be committed to using resources efficiently, thus the less cost conscious they are.

Table 3 presents a summary of the variables which were evaluated by Shields and Young (1994) and Abernethy and Vagnoni (2004), and those which the respective studies identified as probable determinants of cost consciousness. It is possible to briefly explore the nature of the five determinants identified in the table above (right most column). First, cost management knowledge, cost budget participation and informal authority highlight the importance of actors in the institutionalization of cost consciousness. Moreover, cost budget participation is an activity related to M ASs as Tsui (2001, p. 127) states that sophisticated MASs allow managers to use cost related information for participative decisions on resource allocation among operating units. Moreover, as mentioned previously, AISs can be considered as control tools which include MAS information. Furthermore, cost of regulations seems to reflect a type of force external to the organization which, for practical purposes, will be referred to as exogenous force. 
Table 3. Determinants of cost consciousness from Shields \& Young and Abernethy $\&$ Vagnoni

\begin{tabular}{|c|c|c|}
\hline Journal Article & Evaluated Determinants & Determinants according to study \\
\hline $\begin{array}{l}\text { Shields and Young } \\
\text { (1994) }\end{array}$ & $\begin{array}{l}\text { Basic Research } \\
\text { Applied Research } \\
\text { Development } \\
\text { Engineering } \\
\text { Cost of Regulations } \\
\text { Top M anagement Attention to Costs } \\
\text { Economic Culture } \\
\text { Scientific Culture } \\
\text { Cost-based Compensation } \\
\text { Economic-M anagement Experience } \\
\text { Hours of Cost M anagement Education } \\
\text { Cost Knowledge } \\
\text { Cost Budget Participation } \\
\text { Cost Budget Tightness } \\
\text { Cost Budget Changes } \\
\text { Personal Spending Style }\end{array}$ & $\begin{array}{l}\text { Cost M anagement Knowledge }(+) \\
\text { Cost Budget Participation }(+) \\
\text { Cost of Regulations }(-)\end{array}$ \\
\hline $\begin{array}{l}\text { Abernethy and Vagnoni } \\
\text { (2004) }\end{array}$ & $\begin{array}{l}\text { Formal authority } \\
\text { Informal authority } \\
\text { Accounting Information Systems (AISs) }\end{array}$ & $\begin{array}{l}\text { Accounting Information Systems (+) } \\
\text { Informal authority (-) }\end{array}$ \\
\hline
\end{tabular}

Before discussing the inferred determinants which have been associated with cost consciousness, it is important to note that although both studies (Shields and Young, and Abernethy and Vagnoni) use the same instrument to evaluate cost consciousness, one can reasonably argue that the degree of holism embedded in the notion cannot be measured by the seven questions presented by Shields and Young. Nevertheless, these studies do exemplify a way to measure a particular interpretation of cost consciousness.

\section{Inferred Determinants of Cost Consciousness}

The previous section identified two articles in management accounting journals were determinants of cost consciousness were evaluated. The two studies identified cost management knowledge, cost budget participation, cost of regulations, AISs, and informal authority as determinants of cost consciousness where cost of regulations and informal authority represent an indirect relationship with respect to an increase in cost consciousness. Moreover, during the review of the definitions and interpretations of cost consciousness, various determinants came to light including market pressures, emphasis on staff participation, strong incentives, emphasis to costs and cost reductions, implementation of time-based measures, implementation of new and more detailed accounting systems, modifications of accounting practices (e.g. changes in budgeting, cost accounting and financial reporting), cost variance reports, and budgetary control systems. Furthermore, although one author noted that higher reporting frequency did not contribute to cost consciousness, it is not possible to determine if this determinant has an indirect relation with the notion.

From these drivers (both identified and inferred), one can reasonably argue that AISs and budgetary control systems could be considered as management information systems (MISs) as MISs provide managers with a broad range of information to achieve multiple strategic performances (Naranjo-Gil 2009). Labeling these determinants as MISs allows embracing, under the same label, other similar concepts which may be (or may not be) closely related with accounting, hence differentiating itself from the concept of MASs suggested by Macintosh and 
Scapens (1990). Moreover, the modification of accounting practices reflects a use of MASs. Furthermore, one can reasonably argue that cost variances reports represent an output of information systems which are clearly related to accounting (i.e. accounting information systems [AISs]). Hence, I will refer to this label as output of AISs. In addition, other determinants such as staff participation, and incentives highlights not only the participation of organizational actors, but, more precisely, of managers in the process of driving cost consciousness. Finally, market pressures acts as another example of exogenous forces together with the cost of regulation discussed in the previous subsection.

Furthermore, the drivers which have been identified until now only represent 10 of the 171 scrutinized articles in this literature review. Hence, I will now review other articles which might shed light into other possible determinants of cost consciousness and reinforce the role of MASs. As it will become evident I will use the labels previously discussed (i.e. MIS, use of MASs, output of AISs, actor participation, and exogenous forces) in the discussion that follows.

In conducting this exercise I would like to present four sets of quotes. The first set of quotes is the following:

The management accountants believed they were introducing a new sense of cost consciousness amongst engineers through the identification of cost and profit centres, the development of budgetary control, and the monthly reporting of actual costs and revenues compared with budgets. They believed this was supported by formal and informal educational practices developed and organised by the management accounting teams. These included staff briefings in workshop-style seminars regarding the purposes and operations of management accounting systems, supported by a booklet explaining the rudiments of accountancy, an induction process for new managers (mainly promoted), and increasing personal contact with engineers to encourage more financial awareness (Coad and Herbert 2009, p. 184)

Although 'prudent' budgetary goals and other financial targets were set by the accounting function to instill cost consciousness throughout the firm, there was little formal accountability or reward attached to budgetary performance. Only the most senior managers had financially based accountabilities. (M arginson and Ogden 2005, p. 438)

The article by Coad and Herbert (2009) suggests various possible drivers of cost consciousness including the identification of cost and profit centers, the development of budgetary control, the monthly reporting of actual costs and revenue compared with budgets, and educational practices developed and organized by the management accounting teams. Hence, these drivers, similarly to modification of accounting practices, also reflect the use of MASs.

Moreover, the quote by Marginson and Ogden clearly associates the establishment of prudent budgetary goals and financial targets as possible drivers of cost consciousness. These too reflect the use of MASs. Furthermore, other authors have also associated various activities related to MAS as drivers of cost consciousness including conducting product costing and allocating costs (van Helden, 1997).

Hence, there is evidence suggesting that various management accounting scholars share the belief that the use of MASs may act as drivers of cost consciousness. It is important to note that 
these activities are considered as MASs themselves according to Chenhall and Langfield-Smith (1998a).

The second set of quotes is the following:

This unit also received reports on drug costs from the pharmacy and on supplies from the hospital's purchasing unit. It was also possible to access financial accounting data (such as the cost of materials ordered) on the computer in the unit. These numerous reports and improved data systems were seen as having contributed to the increasing cost consciousness of medical professionals. According to the ward sister: In the 1970s we did not consider very much what we consumed, we used what we used without thinking much ... Even in the 1980s . . But then towards the end of the 1980s we started thinking about costs, and now our staff are very cost conscious. (Kurunmäki et al. 2003, p. 130)

In the context of healthcare, casemix accounting systems are involved in attempts to increase the cost-consciousness of clinicians through the rationalization and codification of clinical activity [...] Rather than presenting casemix accounting as simply a tool for rational decision making on the allocation of scarce resources in healthcare, these authors draw upon different literatures in order to present casemix accounting as implicated in the attempted normalization of medical practice through the increased surveillance of clinicians and clinical activity. (Lowe and Doolin 1999, p. 181)

From the quote by Kurunmäki et al., one can infer that improved data systems and reports based on financial data may serve as drivers of cost consciousness. These, furthermore, clearly represent outputs of MISs similar to cost variance reports. Moreover, Nor-Aziah and Scapens (2007) have associated financial reports as drivers of cost consciousness, which, one could reasonably argue, also represent similar outputs of M ISs.

Furthermore, the quote by Lowe and Doolin (1999) suggests that a particular type of MIS (i.e. casemix accounting systems) can drive cost consciousness in an organization. In addition, this quote also indicates the rationalization and codification of clinical activity could be a supportive mechanism for the driving of cost consciousness. It is interesting that the literature review also identified the works by Lehtonen (2007) and Doolin (2004) which also associate casemix accounting systems as drivers of cost consciousness. In addition, other scholars have also associated M ISs as drivers of cost consciousness. For example, Groot and Budding (2004) note that prudent budgetary systems may increase cost consciousness in an organization.

Therefore, these references provide further evidence that various management accounting scholars seem to share the belief that MISs, and reports resulting from MAS may act as drivers of cost consciousness. It is important to note, once again, the conceptual overlaps which are prevalent in the field as, for example, Chenhall and Langfield-Smith (1998a 1998b), consider these reports as M ASs themselves.

The third set of quotes is the following:

Innovative MCS, based on ABCM, were seen as a way to map activities to different capabilities and to associate costs with these activities. On the basis of working with this activity and cost information both formal documentation and the senior management claimed that they expected that the organizations' operational 
management would move from the traditional military effectiveness focus to an orientation that focused on efficiency fostering a cost consciousness. This would enhance understanding of the costs and benefits of different capabilities associated with varying degrees of readiness. (Chenhall and Euske, 2007, p. 211)

An alternative form of team-level accounting control might be target cost management (Tanaka, M., Yoshikawa, T., Innes, J. and M itchell, F., 1993) applied to the team as a whole. While such an approach might encourage greater accountability and cost consciousness among team members, it would also seem likely to restrict their ability to respond to customer requirements. (Nandhakumar and Jones 2001, p. 211)

One could reasonably infer that Activity Based Cost M anagement ( $A B C M$ ) has been associated as a driver of cost consciousness by Chenhall and Euske. Furthermore, Target Cost Management seems to be associated as a driver of cost consciousness by Nandhakumar and Jones, and it involves target costing, an important technique for long-term managing product costs, in particular "setting a target cost to be achieved in the product development process, such that a sufficient profit margin is realized when the product is introduced in to the market" (Dekker and Smidt 2003, p. 293). Both of these inferred drivers represent types of management programs or strategies which use and promote MASs (Baird 2007, Zengin and Ada 2010). I will refer to these as Management Programs for the remaining of this dissertation. Furthermore, other Management Programs have also been noted by other scholars. For example, Järvinen (2006) mentions that Activity Based Costing ( $A B C$ ) may allow organizations to appear cost conscious. Hence, it seems that various management accounting scholars seem to share the belief that Management Programs which use and promote MASs may act as drivers of cost consciousness. However, it is worth noting that these programs have also been considered as MASs by Chenhall and Langfield-Smith (1998a). This, again, highlights the conceptual overlaps.

Moreover, the fourth set of quotes is the following:

However, as the result of the market crash, U.S. securities firms are probably more cost conscious and aware of the need for concern with a revenue mix that will allow them to meet competition, not only at the national level, but in the international sphere as well. (Young 1991, p. 130)

Meanwhile [during the 1837 economic depression], the emergence of a better capitalized and more cost-conscious private sector in arms manufacture offered a more managerial model for Springfield to follow (Tyson, 1990, pp. 56-8). (Hoskin and Macve 1994, p. 7)

However, even the Asian economic crisis did not appear to have an impact on the production orientation within Eagle. Although there was an awareness of the need to be more cost conscious, the economic crisis did not have a significant effect on the demand for gas. (Siti-Nabiha and Scapens 2005, p. 53)

The quote by Young (1991) suggests that a market crash may drive cost consciousness. Similarly, Hoskin and Macve (1994) and Siti-Nabiha and Scapens (2005) identify economic depressions as drivers of cost consciousness. Furthermore, M cDermott et al. (1997) and Masquefa (2008) also provide examples of external forces driving cost consciousness. Therefore, it seems that some management accounting scholars share the belief that economic downturns, and similar types of external forces, may act as possible drivers of cost consciousness. These are part of the category 
named exogenous forces that, according to management accounting scholars, drive cost consciousness.

Summarizing this section, the literature review identified two studies evaluating the possible determinants of cost consciousness. In addition, it was possible to infer various determinants which management accounting scholars have associated with cost consciousness. While some of these drivers refer to M ISs, others refer to activities reflecting a use of MASs, outputs of MISs, $M$ anagement Programmes, exogenous forces, and the role of actors. It is important to note that some of these are considered by Chenhall and Langfield-Smith (1998a) as examples of MASs. However, this dissertation tries to differentiate those drivers by labeling them and categorizing them. The usefulness of this differentiation will be evident in the following chapters. Nevertheless, this exercise has allowed highlighting the driving role of MASs which management accounting scholars have associated with cost consciousness.

\section{Results of Cost Consciousness}

This section focuses on identifying and inferring the possible results which management accounting scholars have associated with cost consciousness.

Although the literature review identified two specific definitions of cost consciousness, and two studies that evaluated possible determinants of a particular interpretation of cost consciousness, it was not possible to identify a single study focusing on evaluating possible results of cost consciousness or of a particular interpretation of the notion. Hence, all of the results which have been identified have, admittedly, required a considerable amount of subjective interpretation.

M oreover, the previous sections have already highlighted, or allowed the reader to infer, results which management accounting scholars have associated with cost consciousness. These results include an acknowledgement of the importance of operation budget (Kurunmäki 1999), better capacity to monitor spending behaviour (Nor-Aziah and Scapens 2007), reduced the autonomy of operations resulting in an impression of lack of trust on accountants or budgeting systems (NorAziah and Scapens 2007), effective control of costs (Trueger 1967); better use of cost accounting data in order to control costs and prevent funding shortfalls and associated sanctions (Geiger and Ittner 1996), better understanding of the association between call times and costs (Andon et al. 2007, p. 287), normalization of practice through increased surveillance of activities (Lowe and Doolin 1999, p. 181), and better understanding of the costs and benefits of different capabilities associated with varying degrees of readiness (Chenhall and Euske 2007). M oreover, the literature review allowed the identification of other possible results including reductions of inventory investment (Tan and Wisner 2003).

Furthermore, it is interesting to notice that most of these results are involved with monitoring or control (better capacity to monitor spending behaviour, better use of cost accounting data in order to control costs and prevent funding shortfalls and associated sanctions, normalization of practice through increased surveillance of activities) which is considered by some authors as one of the main roles of accounting systems (Burchell et al. 1980, Henri 2006).

M oreover, one can clearly separate those results which seem to suggest a positive outcome (e.g. acknowledgement of the importance of operation budget, better capacity to monitor spending behaviour, effective control of costs, better use of cost accounting data in order to control costs and prevent funding shortfalls and associated sanctions); and those which suggest a negative 
outcome (i.e. reduced the autonomy of operations resulting in an impression of lack of trust on accountants or budgeting systems). However, with respect to the negative outcome, it was noted that the causality was not completely clear and it is not possible to be certain if this result was a consequence of the increase of cost consciousness or of the drivers of cost consciousness. Nevertheless, additional examples of negative associated outcomes are present in the literature:

However, approaches to [Horizontal Organization] HO do not seem as concerned about building a cost consciousness as are the proponents of [Activity Based Cost $M$ anagement] ABCM. Cost consciousness is seen to lead to distraction and a myopic vision of the organization. To the advocates of HO, accounting may improve understanding of product costs, although this is by no means certain, and is likely to be useful only for those products that have relatively routine activities. (Chenhall 2008, p. 529)

The quote above clearly identifies distraction and myopic vision of the organization as a possible result of cost consciousness. The author, later notes, that although accounting may improve understanding of product costs, it is probably only useful for those products that have relatively routine activities.

Finally, in certain cases, authors have not been clear about the possible consequences of increasing cost consciousness:

In addition to facing the new retailers' demands for smaller lot sizes, more frequent deliveries and special packaging and products, manufacturers found also that these powerful buyers added to the cost-consciousness that already was riveting the industry as a result of increases in global competition. In response, firms in the power tool industry found it necessary to adopt a host of new practices and techniques to remain competitive. [...] This study documents that significant advances in areas such as cellular manufacturing, CNC equipment, worker training and empowerment, and quality improvement programmes have helped considerably in bridging the gap between low cost and responsiveness. As will be seen, each of these individual techniques works towards the goal of eliminating elements of production trade-offs. (M cDermott et al. 1997, p. 77)

The lack of clarity about the consequences of cost consciousness allows one to wonder if cost consciousness is a result in itself in a similar way as the quote by Singer (1961), presented in the introduction, suggesting that management accounting "is the activity - and its result". A summary of some of the results which were possible to identify appear in Table 4. 
Table 4. Summary of results associated with cost consciousness

\begin{tabular}{|l|}
\hline Positive Results: \\
\hline Enhancement in evaluating alternatives in terms of financial outcomes \\
\hline Better monitoring of spending behaviour of managers \\
\hline Making management accountable in financial terms \\
\hline Increased capability to rationalize and codify activities \\
\hline Better understanding of costs \\
\hline Improvement of different capabilities associated with varying degrees of readiness \\
\hline \\
\hline Negative Results: \\
\hline Distraction or myopic vision of the organization \\
\hline $\begin{array}{l}\text { Generating official management accounts which are perceived by engineers mainly as a control } \\
\text { device }\end{array}$ \\
\hline Restriction of ability to meet customer demand \\
\hline $\begin{array}{l}\text { Reduction of autonym of operation managers and therefore giving them the impression of not } \\
\text { being trusted }\end{array}$ \\
\hline
\end{tabular}

Summarizing chapter 3.2, the literature review identified the use of the cost consciousness notion across various academic disciplines including accounting, healthcare and medicine, education, computer sciences, biology, economy, finance, operations management, business, technology management, sociology and law. Although the notion seems to be used quite frequently in healthcare and medicine related journals, there seems to be no evidence of a conceptual development of cost consciousness in that area (or any other field). Furthermore, 171 peered reviewed articles included in management accounting related journals - where the words cost consciousness and/or cost conscious appear in their text - were scrutinized in an attempt to identify and infer definitions, interpretations, determinants and results which management accounting scholars have associated with the notion. Through this literature review, I uncovered two definitions of cost consciousness and inferred the interpretations of the notion from various quotes. While some authors seem to interpret cost consciousness very narrowly (e.g. as cost reduction), others suggest a more holistic connotation. The latter is clearly in line with the result of the linguistic composition of cost consciousness. Moreover, the literature review also identified two studies which used the same instrument to evaluate possible determinants of a particular interpretation of cost consciousness. Additionally, the scrutiny of the literature review allowed the identification of many other possible determinants of cost consciousness which have been labeled as MISs, use of MASs, output of AISs, Management Programmes, role of actors, or exogenous forces. Hence, it seems that M ASs play a central role in driving cost consciousness in an organization. Finally, the literature review allowed the identification of various results associated with cost consciousness, most of which seem to have positive connotations.

\subsection{Survey Research and Interviews Supporting Conceptual Exercise}

This subchapter describes and analyzes the results of the Survey Research and Interviews which drove my personal interest on the concept of cost consciousness. Although these two methodological strategies do not serve as tools for testing the formulation previously posited, they do provide examples of how empirical research can highlight, perhaps unintentionally, potential for furthering the conceptual development of abstract concepts, used in a particular jargon, which are commonly overseen. 


\section{Survey Research}

The survey research supporting this dissertation was not originally meant to evaluate cost consciousness, but evaluate the state of long-term costing in Finland's manufacturing sector. The study was based on a survey developed and conducted between the years 2007 and 2008 gathering almost 80 responses. The results provide some interesting insights into to the state of long-term costing in Finnish manufacturing companies.

\section{Background of the Survey}

The objective of the survey was to provide an update on the state of long-term costing practices as these provide various benefits and advantages. Barringer and Weber (1996) state that Life Cycle Costing (LCC) - which is an example of a long-term costing - can provide both producers and users with the following:

- Affordability studies to measure the impact of a system or project's LCC on long term budgets and operating results.

- Source selection studies to compare estimated LCC among competing systems or suppliers of goods and services.

- Design trade-offs to influence design aspects of plants and equipment that directly impact LCC.

- Repair level analysis to quantify maintenance demands and costs rather than using rules of thumb such as "...maintenance costs ought to be less than _? percent of the capital cost of the equipment".

- Warranty and repair costs which help suppliers of goods and services and end-users to understand the cost of early failures in equipment selection and use.

- Suppliers' sales strategies can merge specific equipment grades with general operating experience and end-user failure rates using LCC to sell for best benefits rather than just selling on the attributes of low, first cost.

Emblemsvåg (2003) adds by identifying three main purposes for LCC: (1) LCC can be an effective tool for providing decision support in the design and procurement of major open systems, infrastructure, etc..., (2) LCC overcomes many of the shortcomings of traditional cost accounting and can therefore give useful cost insights in cost accounting and management, and (3) LCC has emerged as a design and engineering tool for environmental purposes. As a final note to this discussion, meeting the requirements to fulfill all of the previously mentioned purposes would be very demanding given the versatility of the LCC notion. LCC is the result of the conceptualization of different methods and processes, thus aligning all of these methods to fulfill all of the possible objectives would be a very difficult task to say the least.

The state of long-term cost analyses in Finland has been studied in a handful of studies since the mid-1990s and their results indicate an increase in the use of methodologies for long-term cost management purposes. Lukka and Granlund (1996) published a study where they note that "not a single respondent indicated that any of the new costing methods [including LCC] was actually in use". This result contrasts the adoption of LCC in the USA which was analyzed in a previous study by Jackson and Ostrom (1980) were they state that LCC had been implemented already in $25 \%$ of their respondents, and additionally, $63 \%$ of these had used LCC for five years or more. Hyvönen (2000) states that up to $6 \%$ of Finnish companies used LCC methods, and a later study by Lindholm and Suomala (2005) argues that $66.7 \%$ of Finnish companies were involved with LCC at 
least on an annual basis, where $50 \%$ of these respondents claimed to use LCC for comparing alternative investment options, $41.7 \%$ for estimating products' economic life cycle, and $33.3 \%$ for budgeting purposes. However, it was noted in this latter paper that the intensity of the use of LCC in Finnish companies was still low. The collection of the previously mentioned results, however, needs to be conducted with caution. The methodologies used for gathering the previous results lack coherence (when taken together under consideration) so the results cannot really indicate any reliable trend of the use of long-term cost analyses. Lindholm and Suomala (2005) argue that several explanations can be offered on the disparity of the results of these previous studies: (1)the interpretation of the LCC approach may vary from one study to another, and (2) the adoption of LCC depends on the industry and conducted studies are heterogeneous in that sense. Our study acknowledges these issues mainly by focusing on one major industrial sector and by drawing on a broad notion on costing (cost classification, disaggregation) on a longterm basis rather than specific conceptualizations of LCC or TCO.

The Results of the Study and the birth of the Cost Consciousness interest

The results of our study provide some interesting insights into to the state of long-term costing in Finnish manufacturing companies. Approximately $30 \%$ of the respondents believe that they have good or detailed information about the life cycle costs of the fixed assets which they acquire. This implies that a considerable number of companies are already implementing various longterm costing practices for diverse reasons including the opportunity to make better decisions and profitability assessments.

In the second section of the survey the respondents were asked to evaluate the level of cost consciousness related to their target-product, in other words, they were asked to define themselves as either highly cost conscious, or not cost conscious) with respect to their awareness about the life cycle of their target products. Almost one third of the respondents (32\%) consider themselves as (at least somewhat) life cycle cost conscious related to the target-product. However, $68 \%$ of the respondents admit not being cost conscious in the long-term. A company that belongs to the former, more advanced, group of companies will be referred to as a cost conscious company (CCC) for the remaining part of this thesis. A company that belongs to the latter group will be referred to as a not cost conscious company (NCCC).

In addition, the results show that although most companies still focus primarily on the purchase price of their purchases, CCCs tend to place a more uniform importance on the estimation of all types of costs, when compared to companies NCCCs. This result indicates that companies in CCCS tend to estimate, even in the acquisition phase, all types of costs, including maintenance and disposal costs, more often when compared to companies in NCCCS. Moreover, according to our survey, CCCs tend to have a higher percentage of outsourced maintenance related activities. However, one might argue that the more maintenance related activities a company outsources, the more tools it has to implement costing practices. The important factor is that suppliers have valuable and reliable information regarding maintenance costs, which at the same time, are considered by many as difficult to estimate. This means that by outsourcing maintenance activities and obtaining this hard-to-estimate information, companies not only save themselves the trouble of developing this estimations, but also obtain information that can help them more accurately estimate and monitor the life cycle costs of their fixed assets. Furthermore, our results indicate important findings regarding the supplier-customer relationship. According to our study, while NCCCs tend to think that the information that suppliers provide is either non-existent or not enough, CCCs have a tendency to claim that this information is sufficient. The results suggest 
that companies should be active in the long-term costing analysis, both externally (with the suppliers), and internally (within their organizations).

The results also indicate that the CCCs normally acquire fixed assets with a short length of life, indicating that the difficulty of forecasting and monitoring the cost of a purchase increases with the length of its life. In addition, we observed that the practice of collecting historical data, making a summary of the costs of the fixed assets, and comparing the estimations done in the various phases of the life cycle of the purchases is not common. Finally, the majority of respondents indicated that the need for long-term cost analyses will continue to grow in the future but it still faces various obstacles. Better costing practices, which include methods to collect and process data, are needed to improve the state of long-term costing in Finnish manufacturing companies. Respondents pointed to procedural barriers (e.g. lack of funds or difficulty of implementation) as the main obstacle to implement these types of analyses.

Summarizing this section, the birth of my interest on cost consciousness surged after acknowledging two main issues. First, when being asked about cost consciousness, respondents provided answers. As respondents could choose not to answer questions, their answers suggest that they held an interpretation of cost consciousness which perhaps they believed was shared by other people. Secondly, the division between CCCS and NCCCS provided interesting conclusions, therefore serving as a platform for questioning about what cost consciousness could be and why it was important. As a result, these two reasons drove my interest on the notion. However, although these results suggested the existence of shared interpretations or connotations about cost consciousness among employees in the Finnish manufacturing sector, I believed that it was important to reach a deeper understanding about these possible shared interpretations and connotations. Hence, the following section describes the approach which I took in trying to uncover these shared assumptions.

\section{Interviews: What do you think Cost Consciousness means?}

The previous section described the results of a survey which suggested the existence of interpretations and understandings of the cost consciousness notion by employees of various companies belonging to the Finnish manufacturing sector. In an attempt to identify the different interpretations and understandings on the notion, I conducted interviews in a multinational Finnish manufacturing company's offices in China. The interviews were organized by a group of six people. From the six people, I was the only one interested in the concept of cost consciousness. Therefore, respondents were asked about various topics including employee training, inventory turnover, localization, and, naturally, cost consciousness. The interviews described below served to reaffirm the importance of cost consciousness in an organization and the existence of a holistic view on the notion which includes different but supporting ideas.

\section{Results of the Interviews}

Through interviews, many employees of Metso across different business departments and also employees of Metso suppliers were asked about how they considered cost consciousness to be driven in their organization. Moreover, the discussions also pointed to the obstacles of cost consciousness and suggestions for further development. Table 5 presents the main findings from the discussions about cost consciousness with the different interviewees. 
Table 5. Insights on the drivers of Cost Consciousness according to interviewees

\begin{tabular}{|c|c|c|}
\hline Interviewee & $\begin{array}{l}\text { Company } \\
\text { (division) }\end{array}$ & How can cost consciousness be developed in your organization? \\
\hline Controller & $\begin{array}{c}\text { Metso } \\
\text { (Automation) }\end{array}$ & $\begin{array}{l}\text { - Supporting employee understanding of the relationship between } \\
\text { their actions and manufacturing costs. } \\
\text { - Clarification and measuring financial effects of tasks and targets. } \\
\text { - Establishing and evaluating (monthly) Cost Centers. } \\
\text { - Setting budgets for each cost center. }\end{array}$ \\
\hline $\begin{array}{l}\text { Vice General } \\
\text { Manager }\end{array}$ & $\begin{array}{l}\text { Suzhou } \\
\text { Ishikawa Iron } \\
\text { Manuf. Co. }\end{array}$ & $\begin{array}{l}\text { - Implementing good quality system to ensure quality (quality impacts } \\
\text { costs). }\end{array}$ \\
\hline $\begin{array}{l}\text { Country } \\
\text { M anager Sales } \\
\text { and Service }\end{array}$ & $\begin{array}{c}\text { Metso } \\
\text { (Automation) }\end{array}$ & $\begin{array}{l}\text { - Increasing understanding of how reducing costs affects the ability to } \\
\text { deliver products to clients. } \\
\text { - Determining best location to procure raw materials. } \\
\text { - Control of quality and quality costs. }\end{array}$ \\
\hline $\begin{array}{l}\text { Operations } \\
\text { Director }\end{array}$ & $\begin{array}{c}\text { Metso } \\
\text { (Automation) }\end{array}$ & $\begin{array}{l}\text { - Implementing methods to follow and monitor costs (ERP systems can } \\
\text { help an organization to achieve this). }\end{array}$ \\
\hline $\begin{array}{l}\text { Sourcing } \\
\text { Manager }\end{array}$ & $\begin{array}{c}\text { Metso } \\
\text { (Automation) }\end{array}$ & $\begin{array}{l}\text { - Reducing waste. } \\
\text { - Setting-up and standardizing proper quality standards (Total Quality } \\
\text { Management approach is necessary even if all companies face } \\
\text { different problems). }\end{array}$ \\
\hline $\begin{array}{l}\text { Sales Support } \\
\text { Manager }\end{array}$ & $\begin{array}{c}\text { Metso } \\
\text { (Automation) }\end{array}$ & $\begin{array}{l}\text { - Forecasting, monitoring and monthly reviewing Net Sales, Operating } \\
\text { Profit, and Order Intake. } \\
\text { - Setting up efficient Cost Centers for expense monitoring. These } \\
\text { should provide periodical reports to the company including quality } \\
\text { performance evaluations. } \\
\text { - Helping people understand the importance of costs by showing them } \\
\text { that all actions impact costs in one way or another }\end{array}$ \\
\hline $\begin{array}{l}\text { Engineer } \\
\text { Manager }\end{array}$ & $\begin{array}{c}\text { Metso } \\
\text { (Automation) }\end{array}$ & - Forecasting \\
\hline $\begin{array}{l}\text { Quality } \\
\text { Manager }\end{array}$ & $\begin{array}{c}\text { Metso } \\
\text { (Automation) }\end{array}$ & $\begin{array}{l}\text { - Focusing on quality tools } \\
\text { - Focusing on cost reductions } \\
\text { - Identification of bottlenecks } \\
\text { - Establishing job rotations to make people aware of how the work of } \\
\text { others affect their own work }\end{array}$ \\
\hline $\begin{array}{l}\text { Machining } \\
\text { Manager }\end{array}$ & $\begin{array}{c}\text { Metso } \\
\text { (Automation) }\end{array}$ & $\begin{array}{l}\text { - Localization of tools and procedures } \\
\text { - Setting up Cross Functional Training }\end{array}$ \\
\hline $\begin{array}{l}\text { Logistics } \\
\text { Manager }\end{array}$ & $\begin{array}{c}\text { Metso } \\
\text { (Automation) }\end{array}$ & $\begin{array}{l}\text { - Having a "tough" CEO who minimizes time spent on discussion } \\
\text { - Localization is very important. } \\
\text { - Cost Control should be a priority over Net Sales. In this sense, not too } \\
\text { much attention should be placed on costs related to human resources }\end{array}$ \\
\hline $\begin{array}{l}\text { VP, Technology } \\
\text { Development }\end{array}$ & $\begin{array}{c}\text { Metso } \\
\text { (Automation) }\end{array}$ & - Construction, understanding and analysis of reliable cost structure \\
\hline
\end{tabular}


As the reader may observe, one can identify several ideas expressed by more than one respondent.

- Better understanding of relationship between actions and their effects on costs

- Establishment, evaluation and monitoring of Cost Centers

- Establishment and periodical revision of budgets

- Implementation of good quality systems

- Forecasting

It is important to note that many of the answers presented above were commonly accompanied by moments of doubt and silence. It seemed that most respondents were not expecting to be asked about such an abstract concept as the interviews mainly focused on topics such as the relationship with suppliers, approaches towards training, retaining and recruiting employees, setting career paths, issues with cultural differences, general economic challenges, employee turnover, challenges due to overcapacity, competition, logistics and localization, costing techniques, performance measurements, cost control, and investment methodologies. Hence, following the discussions held during the review of consciousness - and which will follow when reviewing agency (subchapter 4.1) - these results support the idea that cost consciousness reflects, to a great extent, practical consciousness. It is not discursive consciousness because it seems to involve tacit skills and practices which escape discursive explanation. Hence, although people do not dismiss the concept (i.e. they seem to accept the existence of cost consciousness), explaining what it is and what it involves requires considerable effort.

\section{Analysis of Interview Results}

The results of this set of Interviews provide mixed conclusions. On the one hand, no respondents asked the interviewer to clarify the construct for them; therefore, suggesting that all respondents held some kind of interpretation of the notion. On the other hand, the responses were very varied and did reflect, in certain cases, a considerable influence of the position of each employee. For example, while the Quality Manager mentioned that cost consciousness was driven by focusing on quality tools, the Sales Manager stated that forecasting Net Sales could contribute to increasing cost consciousness. Hence, cost consciousness seems to be an organization-wide phenomenon with different connotations to different people. Therefore, the institutionalization of cost consciousness would most likely require concrete applications in different functions or departments of an organization. In other words, and quite bluntly, there is not a "one size fits all" with respect to the institutionalization of cost consciousness.

However, most of the responses deviated naturally from answering what is cost consciousness? towards how cost consciousness may be driven? (e.g. allowing a better understanding about the relationship between actions and their effects on costs as a driver of cost consciousness). Therefore, these results are aligned with the method for conceptualizing cost consciousness used in the literature review where the drivers of cost consciousness provided more space for discussion when compared to its definitions or interpretations. Furthermore, this represents a good approach to the conceptual development by deviating from the considerable challenges in defining cost consciousness.

In addition, various responses from the Metso employees were aligned with some of the drivers identified in the literature review. These include: (i) implementation and modification of product budgeting systems (mentioned by the Controller); cost and profit center identification 
(mentioned by the Controller, and the Sales Support Manager), increasing cost management knowledge (mentioned by the Controller, the Country Manager of Sales and Service, the Sales Support Manager, the Quality Manager, and the Vice President of Technology Development), implementation of modern cost management system (mentioned by the Operations Director), and the use of financial supporting measures (mentioned by the Sales Support Manager). Moreover, although none of the respondents identified exogenous forces as a determinant of cost consciousness, some of them (including the Logistics $M$ anager, and the Country M anager of Sales and Service) did highlight the need to improve costing capabilities given the increase in competition and prices of raw materials and labour in China.

Summarizing this subchapter, the results of the survey triggered my interest on cost consciousness as the respondents' answers suggested a shared interpretation of the notion. Hence, these results also support the idea of cost consciousness reflecting shared assumptions and beliefs due to, perhaps, its broad but restricted linguistic composition. M oreover, the results of the set of interviews are aligned with many of the findings in this chapter, particularly with respect to the driving role of MASs which management accounting scholars have associated with cost consciousness. Finally, the results of the survey highlighted other drivers such as the need to have a tough CEO, establishing rotations to increase understanding of costs and their relationships with activities, and the setting up of cross functional training programs. These drivers, clearly represent the role of actors which had been previously highlighted as a driver of cost consciousness.

\subsection{Synthesis of Conceptual Exercise}

This dissertation has argued that the linguistic composition of cost consciousness should be considered as a strength as it provides the notion with a broad conceptual realm which, nevertheless, is defined (i.e. narrowed) to serve fields including management accounting. While cost consciousness's linguistic head (i.e. consciousness) provides it with a, perhaps unwanted, abstract nature, cost consciousness's modifier (i.e. cost) allows the notion to serve fields where costs are important. One of these fields, clearly, is management accounting were MASs are a topic of major interest.

Moreover, the results obtained from the survey and the set of interviews suggest that people seem to assume shared assumptions with regards to the notion. In other words, their answers suggest that people assume that others have a similar understanding with respect to the notion. However, when reviewing these answers, it is clear that cost consciousness has different connotations in different environments. In addition, the results of these two methodological strategies supported the relationship between cost consciousness and practical consciousness which might explain the difficulty in providing a discursive explanation of what cost consciousness is and how it can be driven.

With respect to the definitions and interpretations, the literature review identified only two management accounting related articles which provided seemingly explicit definitions of cost consciousness. These definitions refer to economic judgment of operations (Kurunmäki, 1999) and economic efficiency (Nor-Aziah and Scapens, 2003). Although both definitions where vague, they suggested that the notion held a very holistic conceptual realm. Moreover, these interpretations also suggested that cost consciousness could characterize individuals and collectives. In addition, given the low number of definitions, the conceptual exercise was also 
based on inferring the interpretations which other authors suggested in the remaining 169 articles. Although some authors seem to suggest a narrow conceptual realm by interpreting cost consciousness as cost reduction or cost containment, others reflected a very holistic and wide concept. As explained, the linguistic composition of the notion explains its holistic nature.

Moreover, with respect to the interpretations of cost consciousness, this dissertation has been inspired by the quote by Dekker (2004) - presented in the introduction - which allowed a platform for pondering on cost consciousness's possible relationship with organizational culture. Actually, the literature review on cost consciousness also identified some associations between the notion and organizational culture; some of which were very explicit. As explained by Hofstede (1998) and Järvenpää (2007), multiple cultures and subcultures may exist in one organization or even in a single subunit of an organization. M oreover, Wilkinson et al. (1996, p. 69) argue that modern organizations "are becoming more culturally diverse than they have ever been". However, I would not like to equate the term institution to subculture as this could result in conceptual overlaps between institutional theory literature and organizational culture vocabulary.

Hence, with respect to the definitions and interpretations of the notion, although I acknowledge the existence of narrow conceptions of cost consciousness (i.e. cost reduction), I believe that the results explored in this chapter suggest that cost consciousness represents a very holistic conceptual notion. Hence, for the purposes of this dissertation, I will dismiss the narrow conceptualizations (e.g. cost reduction and cost containment).

Furthermore, the literature review also focused on identifying and inferring the drivers or determinants which management accounting scholars have associated with the notion of cost consciousness. The review identified only two studies (Shields and Young 1994, and Abernethy and Vagnoni 2004) evaluating the drivers of a particular interpretation of cost consciousness (as they used the same instrument for the evaluation). In addition, it was also possible to infer drivers which management accounting scholars have associated with cost consciousness. Interestingly, many of these drivers were closely related to MASs (e.g. MISs, output of AISs, use of MASs, and Management Programmes which probably require MASs). Hence, it was clear that management accounting scholars associate MASs as a driving force of cost consciousness. This conclusion would serve greatly the theorization exercise as MASs take a central role when integrating institutional and structuration theories in management accounting studies.

However, it is important to consider the causal relationships between these types of drivers and cost consciousness. Within this respect I would like to make two main observations. The first observation is related to the M anagement Programmes such as Activity Based Cost Management (ABCM) and Target Cost M anagement (TCM) which have been associated as possible drivers of cost consciousness. The interviews in M etso identified the belief that Quality Management could also be considered as a driver of cost consciousness. Therefore, a wide variety of strategies seem to be regarded as useful tools for furthering cost consciousness in an organization. Other options could include, for example, strategic cost management or structural cost management, the latter allowing accountants to embrace higher strategic positions in an organization (Anderson and Dekker 2009). Hence, I am wary of the causal relationship between these strategies and the institutionalization of cost consciousness. Although these Management Programmes might involve MASs which may drive cost consciousness, they might also fail to do so; therefore, I suggest not considering M anagement Programmes as drivers of cost consciousness. 
Furthermore, the second observation refers to what has been referred in this dissertation as a MISs (i.e. AISs, casemix accounting systems, ERP systems, data systems, and product budgeting systems). According to Salehi et al. (2010), the objective of AISs is to collect and record data and information regarding events that have an economic impact upon organizations and the maintenance, processing can communication of information to stakeholders. M oreover, casemix accounting systems are considered as systems for managing and controlling activities in relation to the consumption of resources (Lowe and Doolin 1999). Moreover, Lowe and Doolin also mention that casemix accounting systems also facilitate management philosophies, techniques leading to improved scheduling of activities, better delegation of budgetary activities to managers, and better increased budgetary participation. Here again rises one interesting relationship as Shields and Young (1994) identified cost budget participation as a possible of cost consciousness. Hence, one could wonder if what makes casemix accounting systems a possible driver of cost consciousness is the fact that it might increase budget participation in an organization. Tsui (2001, p. 127), seems to agree with this relationship, as the authors states that sophisticated MASs allow managers to use cost related information for participative decisions on resource allocation among operating units. Therefore, I suggest that although these M ISs should be considered as possible drivers of cost consciousness, the causality should be assessed with caution.

In addition, the review also identified other exogenous forces which have been associated to cost consciousness most of which reflect negative financial circumstances such as market crashes and economic turndowns. Furthermore, these results also suggested the important role of actors in the driving of cost consciousness (e.g. having a "tough" CEO who minimizes time spent on discussion, developing inter-functional training programs).

Moreover, from prior literature it was also possible to identify and infer both positive and negative results associated with cost consciousness. A positive association, however, seems to be more common among authors. The identified result which seems to be the broadest from a conceptual perspective refers to "a better understanding of costs". The other positive results (e.g. enhancement of capabilities to evaluate alternatives of financial outcomes, better monitoring of spending behaviour, and increased capacity to rationalize activities) could be interpreted as results of a better understanding of costs.

The results of this literature review are presented in Figure 7. The red signs in the figure identify those elements which, although acknowledged, I suggest not to consider for the theorization exercise. Furthermore, the yellow signs refer to elements which could be considered for the theorization exercise, but with caution as their causal relationship with cost consciousness is unclear. Furthermore, for clarification purposes, while the two red signs and the yellow sign on the block named Management Accounting Systems apply for all the elements of the each block, the yellow sign on the block named Negative Results suggests caution only with respect to that particular result (i.e. reduces the autonomy of operation managers and therefore giving them the impression of not being trusted). 


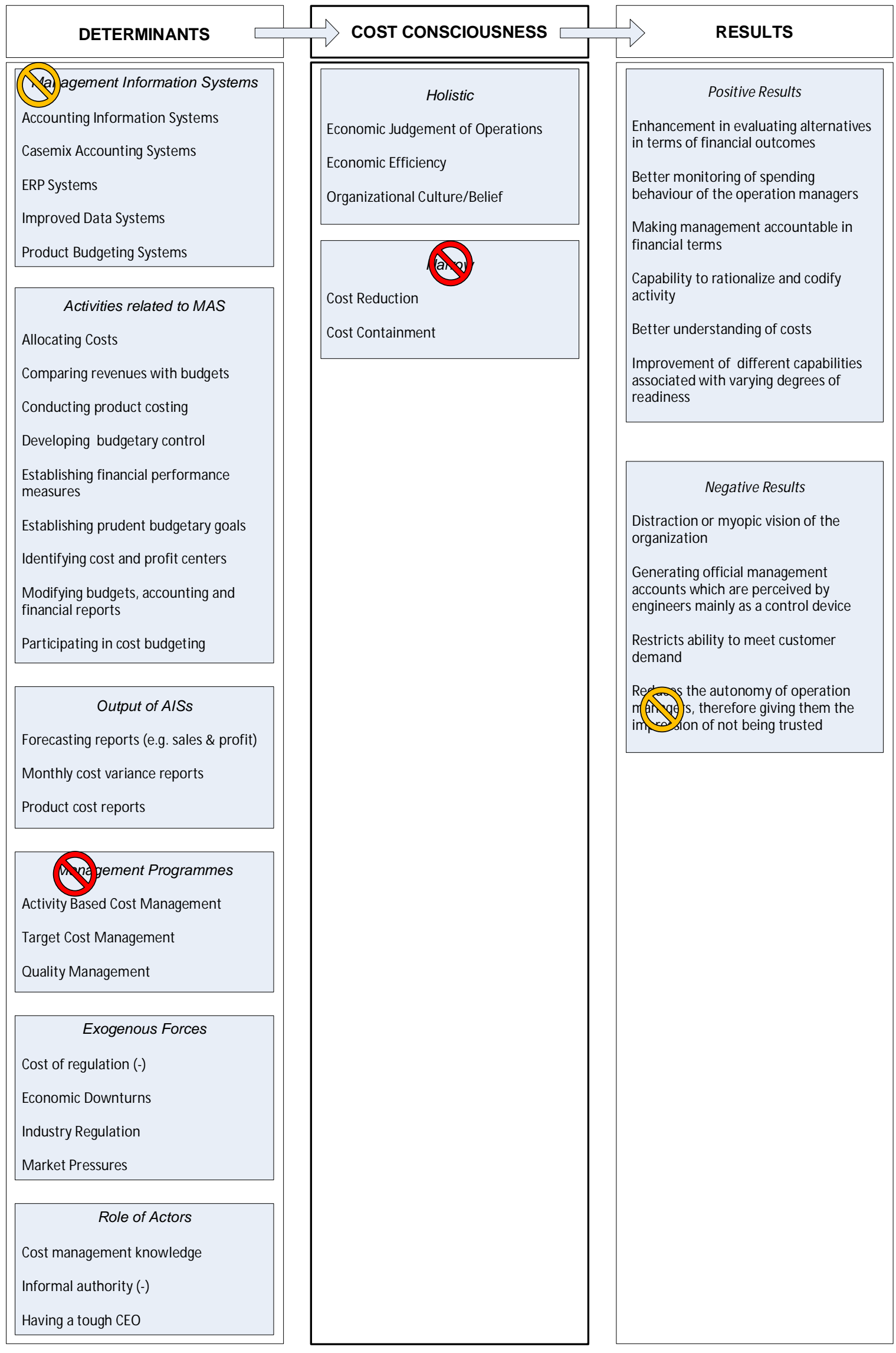

Figure 7. Summary of determinants, interpretations and results of cost consciousness

Summarizing Chapter 3, the first contribution of this dissertation has been approached and constructed to a great extent by taking three main steps which have, simultaneously, structured 
this chapter. First, given the importance of cost consciousness's linguistic composition in arguing for the importance of this notion's conceptual development, subchapter 3.1 focused on providing overviews of the notions of consciousness (which acts as cost consciousness's linguistic head), and management accounting practices (many of which are intricately related to costs) From these discussions, the central role of MASs in the fields of management accounting and accounting was highlighted. Second, subchapter 3.2 analyzed the results of the literature review on cost consciousness which suggest that the notion has been widely used across various academic disciplines, a finding which contrasts a very low number of studies defining or evaluating the concept. Moreover, this chapter allowed the identification of some assumptions and beliefs which are shared among some management accounting scholars with respect to drivers, interpretations, and results related to cost consciousness. From these results, one can reasonably argue that cost consciousness is considered in most of the cases as a very broad phenomenon. Moreover, although cost consciousness has been considered as an organizational culture, I propose that the notions of organizational culture and institution should be differentiated. In addition, MASs have been associated by various scholars as a driving force behind cost consciousness. Third, subchapter 3.3 reviewed the results of the survey and set of interviews that serve as support to this conceptual exercise. These results supported the role of MASs in the driving of cost consciousness and reinforced the relationship between cost consciousness and the practical type of consciousness.

Finally, given the conceptual broadness of M ASs taken in this dissertation, it is important to take stock of the way in which MASs act as drivers of cost consciousness. Therefore, the following chapter explores structuration theory, institutional theory, and the Burns and Scapens Framework (BSF) in order to build the foundations for the theorization on the institutionalization of cost consciousness. 


\section{The Supporting Theoretical Realm: Institutional Theory}

This dissertation is concerned with furthering the understanding of cost consciousness. One of the contributions of this study is to conduct a theoretical exercise that identifies and analyzes the organizational elements and forces which may collaborate in the institutionalization of cost consciousness (i.e. this dissertation's second contribution).

Hence, the theorization exercise should be supported by a theory concerned not only with institutions, but also with organizational actors, processes, and other elements and mechanisms. Moreover, as this dissertation is concerned with management accounting, it would be convenient if management accounting scholars would recognize such a theory as a useful tool in developing contributions in the field.

Zucker (1987) explains that institutional theory provides a rich and complex view of organizations with particular focus on the influences which external sources (e.g. the state) and intraorganizational forces place on them. Moreover, institutional theory can also allow tracing "the emergence of distinctive forms, processes, strategies, outlooks, and competences as they emerge from patterns of organizational interaction and adaptation" (Selznick 1996). In addition, many social sciences, including history, sociology, economics, and political science, have placed interest on institutions, and, as a result, there has been an institutionalist tradition across the social sciences realm (Carruthers 1995, Goodin 1998).

Furthermore, institutional theory has allowed social sciences to enter "a phase of more deliberate development, accompanied by efforts aimed at self-assessment and consolidation" (Scott, 1987). In fact, institutional theory has been used in various studies related to the management accounting field (see examples in Granlund 2001; Soin et al. 2002; Baxter and Chua 2003; Bennett et al. 2004; Hassan 2005; Seal 2006; Kholeif et al. 2007; Englund and Gerdin 2008; ter Bogt 2008; Busco 2009; Järvenpää 2009; Tucker 2010; Moore 2011; van der Steen 2011). M oreover, inside the realm of institutional theory, the Institutional Economics branch has served various management accounting studies (see examples in Burns and Scapens 2000; Siti-Nabiha and Scapens 2005; Abrahamsson and Gerdin 2006; Lukka 2007; Johansson and Siverbo 2009; Quinn 2011; ter Bogt and van Helden 2011).

In addition, one management accounting related topic which has favored from these theoretical realms is management accounting change (see examples in Collier 2001; Granlund 2003; Ribeiro and Scapens 2006; Busco et al. 2007; Englund and Gerdin 2008; Johansson and Siverbo 2009; ter Bogt and van Helden 2011). M oreover, management accounting change has been approached, in various occasions, with the use of the Burns and Scapens Framework drawing from OIE, NIE, and NIS (see examples in Burns and Scapens 2000; Burns 2000; Siti-Nabiha and Scapens 2005; Guerreiro et al. 2006; Coyte et al. 2010; Busco and Scapens 2011; Quinn 2011; Wanderley et al. 2011).

The Burns and Scapens Framework (BSF) "explores the complex and ongoing relationship between actions and institutions and demonstrates the importance of organizational routines and institutions in shaping the processes of management accounting change" (Burns and Scapens 2000 , p. 3). Hence, there is reason to believe that this framework may provide the necessary support for conducting this study's theoretical exercise. 
Therefore, not only does institutional theory provide a theoretical realm which suits the aims of the second contribution of this dissertation; but, furthermore, Institutional Economics, and the BSF in particular, seem to be a good candidates in providing the management accounting perspective which is needed.

Before reviewing Institutional Economics, however, it is important to clarify certain concepts central to Institutional Economics including agency and organizational routines. Moreover, the notion of institution, as considered in Institutional Economics, has some strong similarities with structure as it is understood in structuration theory. As structuration theory also takes a thorough look into agency and routines, a review on this theory would allow better understanding of OIE and NIE. Furthermore, as structuration theory has been used in various studies related to management accounting (see examples in Roberts and Scapens 1985; Macintosh and Scapens 1990, 1991; Boland 1996; Granlund 2003; Jack 2005; Busco et al. 2007; Langfield-Smith 2008; Coad and Herbert 2009; Busco and Scapens 2011), this review maintains the management accounting perspective of this dissertation.

Hence, subchapter 4.1 provides a literature review on Anthony Giddens's structuration theory which not only provides clarification on notions such as organizational routines, but also provides a platform for better understanding other central tenets of institutional theory (e.g. institutions). Furthermore, subchapter 4.2 describes the main tenets of Institutional Economics including evolutionary theory, rules, routines, and institutions. In addition, subchapter 4.3 conducts a review on OIE and NIE aiming to assess which of these two branches allows a phenomenon like cost consciousness to have a central role. Finally, subchapter 4.4 discusses the Burns and Scapens Framework (BSF) which draws mainly on OIE, but also on NIE and New Institutional Sociology (NIS). In this final subchapter, a review on management accounting change is also presented.

\subsection{Structuration Theory}

This subchapter focuses on exploring some aspects of Giddens's structuration theory which can be considered to be relevant to Institutional Economics and the overall objectives of this dissertation.

Recently, Englund et al. (2011) celebrated the " 25 years of Giddens in accounting research" by reviewing the use of structuration theory in the accounting field, a move championed by Roberts and Scapens (1985) who argued for the use of structuration theory as a useful framework for understanding accounting as an organizational and social practice. The structuration theory of British sociologist Anthony Giddens $(1979,1984)$ aims at understanding the relationship between the activities and interplay of knowledgeable human actors and social structures in the production, reproduction and regulation of any social system (Macintosh and Scapens 1990, Busco 2009.) The theory states that the domain of any social science is composed by a complex relationship between the experience of agents and structures, the latter being conceived as properties of systems.

This subchapter is divided into various sections. The first section highlights the benefits of structuration theory by exploring how it tackled the established orthodox consensus on sociology on the 1970s. The second and third sections review some of the central notions of the theory including systems, structures, agency, the duality of structure, routines, and power. The fourth 
section analyzes the relationship between structures and institutions. Finally, the fifth section discusses the relationship between the duality and modalities of structure.

\section{The Need for a Theory of Structuration}

Giddens's Central Problems of Social Theory: Action, Structure and Contradiction in Social Analysis, is a collection of separate papers "examining the residue of nineteenth-century European social theory for contemporary problems of the social sciences" (Giddens, 1979, p. 1). The starting point of this work was Giddens's acknowledgement of the "state of disarray" that characterized social sciences at the moment (Giddens, 1979, p. 235). Giddens states that the type of orthodox consensus of sociology, held until the middle 1960s, involved two main strands. The first strand refers to the theory of industrial society which opted for a bipolar contrast between traditional society and industrial society concluding that no form of socialist society could be distinctively different from a capitalistic one - Giddens (1979, p. 235) refers to this view as 'archaic'. The second strand, of a more abstract character, refers to the prevalence of functionalism and naturalism in social sciences. Functionalism regards biology as a proximate model for sociology as both disciplines, it is argued, deal with systems rather than aggregates. However, Giddens argues that "models of biological systems, especially those tied to a notion of homeostasis, will not suffice to illuminate the key issues posed by the analysis of social systems". Moreover, naturalism referred to the idea that the logical frameworks of natural and social sciences where the same, a position closely connected to the functionalist view described above.

In an attempt to tackle the state of disarray, Giddens constructed a comprehensive theory (i.e. structuration theory) which disrupts the vis-à-vis origins of sociology and naturalism, reformulates the philosophy of language, enhances the model of social science, and illuminates the role of agency in sociology. A considerable amount of Giddens' efforts are allocated in constructing (or reconstructing) the concept of agency aiming to challenge the "imperialism of the subject" which functionalism and previous structuralism proposed.

Giddens simplifies and summarizes structuration theory in the following passage:

The basic domain of study of the social sciences, according to the theory of structuration, is neither the experience of the individual actor, nor the existence of any form of societal totality, but social practices ordered across space and time. Human social activities [...] are recursive. That is to say, they are not brought into being by social actors but continually recreated by them via the very means whereby they express themselves as actors. In and thorough their activities agents reproduce the conditions that make these activities possible. However, the sort of 'knowledgeability' displayed in nature, in the form of coded programmes, is distant from the cognitive skills displayed by human agents. It is in the conceptualizing of human knowledgeability and its involvement in action that I seek to [...] (Giddens 1984 , p. 2)

From this previous passage, one can rescue the attention towards the complex relationship between actors and social activities/practices which Giddens tries to distant from naturalistic views. Activities and practices exist across space and time, and are recreated by actors. Hence, priority is given neither to the individual, nor to society in general; rather to the interactions in space and time. 
However, agency is only one of the main elements which are central to structuration theory. Systems, structures, and the process of structuration interact with agency in constructing the conceptual base of structuration theory. Although this dissertation will not address most of the theory, a review on the theory's conceptual base will allow a better understanding of some of the central tenets of OIE and NIE.

\section{On Systems, Structures, Agency, and Structuration}

Giddens's structuration theory aims, among many objectives, to formulate the relationship between social systems, structures, and agents. One of the difficulties in achieving this objective relates to the considerable challenges in defining these notions given their abstract and fuzzy character. With respect to this, Sewell $(1992$, p. 5) states:

Most strikingly, "structure" - the central term of [Giddens's] theory - remains frustratingly underspecified. Unlike most social scientists, he does not leave the term completely undefined and simply allow it to do its accustomed magical work in his readers' minds.

While acknowledging Sewell's statement, Giddens does present definitions of these notions; and, if one wishes to review structuration theory, it is important to review these definitions. However, I do agree that the definitions are quite abstract and subject to debate.

An overview of Systems, Structures, Agency, and Structuration

I have chosen to begin this subsection by presenting the definitions of these notions found in Giddens's 1984 central work, The Constitution of Society:

Systems

Reproduced relations between actors and collectivities, organized as regular social practices (p. 25).

The patterning of social relations between actors and collectivities across space and time, understood as reproduced social practices. Social systems should be regarded as widely variable in terms of the degree of 'systemness' they display and rarely have the sort of internal unity which may be found in physical and biological systems (p. 377).

Structure

Rules and Resources, or sets of transformation relations, organized as properties of social systems (p. 25).

Rules and resources recursively implicated in the reproduction of social systems. Structure exists only as memory traces, the organic bases of human knowledgeability, and as instantiated in action (p. 377).

Structures are rule-resource sets, implicated in the social articulation of social systems (p. 377).

Structuration Conditions governing the continuity or transmutation of structures, and therefore the reproduction of social systems (p. 25).

Agency

Refers not to the intentions people have in doing things but to their capability in doing things in the first place. Agency concerns events of which an individual is the perpetrator, in the sense that an individual 
could, at any phase in a given sequence of conduct, have acted differently. (p. 9)

Duality of Structure Structure as the medium and outcome of the conduct it recursively organizes; the structural properties of social systems do not exist outside of action but are chronically implicated in its production and reproduction (p. 374)

Furthermore, as structures only exist as structural properties of social systems enabling the binding of time and space in social systems, social systems are not structures (they include structures), and may be considered as 'structured totalities' (Giddens, 1979, pp. 65-73). Structures, hence, seem to be necessary properties for the comprehensive existence of systems. Without them, systems would not exist, at least not in the way that they are conceived when being constituted by structures. Sewell (1992, p. 6) interprets Giddens's social systems as empirically observable, and relatively bounded social practices that encompass not only what social scientists consider as society but also social units of greater or more limited scope when compared to, for example, the state. According to Sewell, the capitalist world system is an example of a social unit of greater scope, and the neighborhood community reflects a social unit of a more limited one. Therefore, the nation of Finland may be considered as a social system. M oreover, the Finnish language may be considered as a structure. One can, indeed, differentiate the nation of Finland from the Finnish language; however, one cannot separate them without disintegrating the present conception of Finland as a nation. Moreover, this discussion also suggests that there are social systems within social systems (e.g. societies including states; states including neighborhood communities).

Furthermore, systems, structures, and agency function in a complex relationship. On the one hand, agents draw on structures to interact with one another. On the other hand, simultaneously during this same process of interaction, agents constitute (or reproduce) structures and therefore reproduce social systems across space and time ${ }^{22}$. Moreover, if enough powerful agents act (interact) in innovative ways, then the structures which feed their interaction might mutate (or transform) as opposed to reproducing mirrored copies of the structures. Structures are, therefore, both the medium and the outcome of the social systems, together with the practices and activities that agents recursively conduct. This process creates a duality ${ }^{23}$ between the two phenomena (agents and structures) which is referred to as the duality of structure. "The duality of structure thus connects the reproduction of systems across time-space with the production of situated interactions" (Englund et al. 2011, p. 495).

Moreover, the continuation or transformation of structures discussed above refers to the structuration process, most commonly referred to simply as structuration ${ }^{24}$. Giddens $(1984$, p. 25) defines structuration as "the conditions governing the continuity or transmutation of structures, and therefore the reproduction of social systems". In other words, structuration signifies the ways in which structures and social systems are produced, reproduced and transformed during social interaction where knowledgeable actors draw upon rules and resources (i.e. structures) in the diversity of action contexts (Giddens 1979, p. 66; 1984, p. 25). "Structuration refers to the

\footnotetext{
${ }^{22}$ While Busco $(2009, p .250)$ says that the systems are what is produced and reproduced; Macintosh and Scapens (1990, p. 457) claim that it is the structures which are reproduced. Giddens $(1979$, p. 66) explains that structuration is the transformation of structures, and therefore reproduction of systems.

${ }^{23}$ Not to be mistaken with dualism - this would suggest two independent sets of phenomena. Agents and structures represent two sides of the same coin (Giddens, 1984)

${ }^{24}$ The word process suggests that structuration is not a steady state (Sewell, 1992, p. 4)
} 
'ongoingness' (durée) of social systems, involving both continuity and change. To study structuration is to study the conditions governing their (re)production" (Englund et al. 2011, p. 495). By knowledgeable, Giddens suggests that agents are people who know what they are doing and know how to do it (Sewell, 1992, p. 4).

The discussion so far aimed at providing an overview of systems, structures, and agency; together with a simplified explanation on some of the relationships between these. However, it is important to dig deeper into these notions, not only for better understanding of the structuration theory, but also, for example, to uncover the way in which routines are introduced into this analysis. Routines, particularly, play an important role on agency as it is understood in structuration theory.

\section{A Closer Look On Agency, Consciousness, Routines, and Power}

\section{On Agency and Consciousness}

Giddens's (1984, pp. 2-14) conception of agent seems to find its starting point in the description of what makes us knowledgeable. In doing so, he invokes the reflexive form of the knowledgeability of human agents as being "deeply involved in the recursive ordering of social practices" (p. 3).

Therefore, Giddens urges the reader not to understand reflexivity as self-consciousness, but rather as "the monitored character on the ongoing flow of social life [...] grounded in the continuous monitoring of action which human beings display and expect others to display"; hence explaining why we are purposeful and, therefore, both have and (if required) can elaborate discursively reasons for our actions. Moreover, the reflexive monitoring of action depends upon the rationalization of action which is the "principal basis upon which the generalized 'competence' of actors is evaluated by others" (p. 4). In addition, motivation is also central to Giddens understanding of actors: "If reasons refer to the grounds of action, motives refer to the wants which prompt it [...] Motivation refers to the potential of action" (p. 6).

Giddens uses his stratification model of action to explain the relationships between reflexive monitoring, rationalization and motivation of action (Figure 8). First, the reflexive monitoring of action involves the everyday action of individuals, particularly with respect to how individuals routinely monitor their own actions and the actions of other individuals. Giddens argues that the distinctive feature about the reflexive monitoring action, when compared to animal behaviour, is the accountability of human action, meaning that "the accounts that actors are able to offer of their conduct draw upon the same stocks of knowledge as are drawn upon in the very production and reproduction of action"(Giddens, 1979, p. 57). Second, the reflexive monitoring of action acts against the background of the rationalization of action that refers to how actors maintain a continuing 'theoretical understanding' of the grounds of their activity as it is expected for actors to be able (usually) to explain most of what they do. Third, motives supply the plans and programs within which a range of conduct is enacted Giddens (1984, p. 6). Figure 8 suggests that acts have unintended consequences which may systematically feed back to become the unacknowledged conditions of human action. Finally, Giddens explains that purpose (together with motives, reasons, and intentions) "occurs as a durée, a continuous flow of conduct" (p. 3) explaining the recurrence that might be observed in the stratification model of action. 


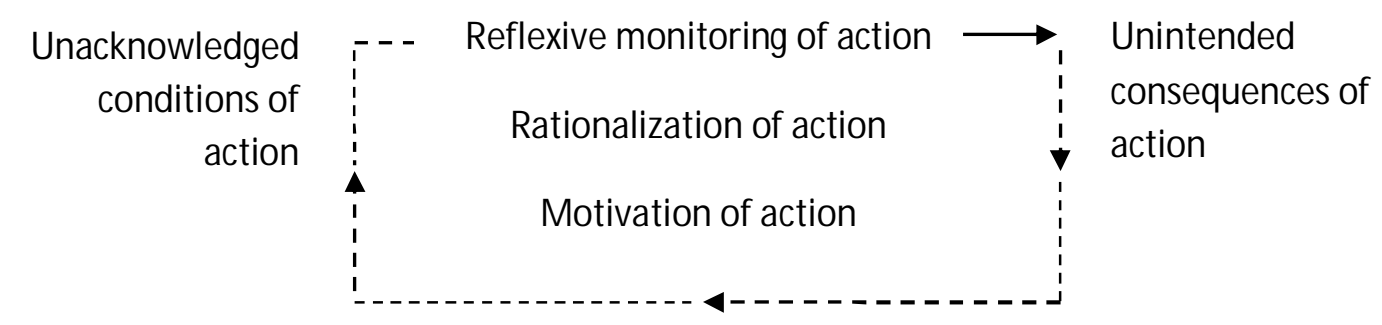

Figure 8. The Stratification M odel of Action (Giddens, 1984, p. 5)

Moreover, intentions ${ }^{25}$, reasons, and motives introduce the discussion on consciousness and unconsciousness. Although agents are normally capable of reporting discursively about both their intentions and reasons, motives are not that often explainable. It would be impossible for agents to pause, reflect and make conscious choices about their behaviour for every single event and situation which they encounter (Macintosh and Scapens, 1990). In order to cope, individuals access two levels of their consciousness ${ }^{26}$ : (1) the discursive level, where agents give reasons for and rationalize about what they do in social settings; and (2) the practical level, where agents rely on implicit stocks of knowledge in relation to how to act and how to interpret events and the actions of others. In other words, practical consciousness is "tacit knowledge that is skillfully applied in the enactment of courses of conduct, but which the actor is not able to formulate discursively" (Giddens, 1979, p.57). The difference between practical and discursive consciousness is not always very clear. However, Giddens argues, there is a clear difference with respect to unconsciousness:

There is no bar between these [discursive and practical consciousness], however, as there is between the unconscious and discursive consciousness. The unconscious includes those forms of cognition and impulsion which are either wholly repressed from consciousness or appear in consciousness only in a distorted form. Unconscious motivational components of action, as psychoanalytic theory suggests, have an internal hierarchy of their own, a hierarchy which expresses the 'depth' of the life history of the individual actor. (Giddens 1984, p. 4)

Most of Giddens's focus on structuration theory deals with the two types of consciousness. Unconsciousness, however, as the following subsection mentions, takes an important role in the creation of human identity.

\section{On What M akes Us Humans and How Routines Support Us}

This subchapter aims not only at describing in more detail Giddens's understanding on agency, but also on explaining agency's relationship with organizational routines. I will now focus on the second aim and, in achieving this, it is useful to briefly refer to Berger and Luckmann's (1966) The Social Construction of Reality as it provides valuable insight into the relationship between agents and structures. Berger and Luckmann open their chapter on institutionalization by discussing on the relationship between organisms and activity. The authors (p. 47) explain that humans occupy a peculiar position in the animal kingdom as there is no human-specific environment contrasting the existence of specific environments for other animals. In other words, while there

\footnotetext{
${ }^{25}$ An intentional act is one which its perpetrator knows, or believes, will have a particular quality or outcome and where such knowledge is utilized by the author of the act to achieve this quality or outcome (Giddens, 1984, p. 10)

${ }^{26}$ This view of consciousness and unconsciousness has no direct relation with the notion of cost consciousness in this work.
} 
is some kind of parrot-world and moose-world, there is no similar kind of human-world partly because humans are not restricted, as parrots and moose are, with respect to their predetermined biological equipment and geographical limitations (e.g. while parrots may live freely in some coastal areas in Colombia, they would probably not survive anywhere [freely] in Finland for a long period of time). Therefore, on the one hand we have non-human animals living in close-worlds characterized by a sort of perfect structure between them and their surrounding environment. On the other hands we humans having an imperfect structure with a surrounding environment which characterizes what Berger and Luckmann refer to as world-openness. Our biological constitution, however, although certainly enables and limits us to a certain extent, does not explain the great range of activities which we humans engage in (e.g. passing from a nomad to an agricultural society). "The peculiarity of man's biological constitution lies rather in its instinctual component" (Berger and Luckmann 1966, p. 48). The key, Berger and Luckmann suggest, lies in the fact that - in contrast to other higher mammals - humans at birth have an underdeveloped instinctual organization which continues to develop as people interrelate from within the environment during the first years of life. Moreover, as this environment is both natural and human, a person interrelates with a specific cultural and social order which is mediated to that person by others, and which, furthermore, socially determines the person's organismic development. Therefore, through this after-birth-interrelation, humans construct their own nature, or, in other words, humans produce themselves (Berger and Luckmann, 1966, p. 49). In addition, the human self is also formed during the first years of life; therefore, its understanding can only be achieved in relation to both the organismic development and the social processes that the human encounters. Moreover, and perhaps more excitingly, the development of the human organism is related to an eccentric relationship between the organism and the self where a person's experience of him/herself balances between being and having a body. The importance of this eccentricity cannot be sufficiently underlined as it "has certain consequences for the analysis of [any] human activity as conduct in the material environment and as externalization of subjective meanings" (Berger and Luckmann 1966, p. 50). Furthermore, a person's self production is always a social enterprise as the human environment is product of social interaction. Humans, while interacting, and as an anthropological necessity, externalize themselves in creating a human environment and a social order, the latter given to humans neither biologically nor in the natural environment. "Both in its genesis (social order is the result of past human activity) and its existence in any instant of time (social order exists only and insofar as human activity continues to produce it) it is a human product" (Berger and Luckmann 1966, p. 52). Interestingly, in discussing consciousness and the self, Flanagan (1992, p. 195) states:

In sum, at one end of the spectrum there is the self-consciousness involved in having a subjective experience. At the other end of the spectrum is the sort of selfconsciousness involved in thinking about one's model of one's self, or as I shall say for simplicity, the self. Unlike the capacity to be self-conscious in the first sense, the self is not something that we are born with. Maturation, living in the world, being socialized in a life form, and acquiring certain ways of thinking are required before one can start to build the complex set of representations that, taken together, come to constitute the narrative self.

Routines can now be introduced into this discussion. Berger and Luckmann note that social order is not only caused by biological constants, but also by the habitualization of all human activity whose meanings “become embedded as routines in [the human's] general stock of knowledge, taken for granted by [humans] and at the hand of [the human's] projects into the future" (Berger 
and Luckmann 1966, p. 53). Furthermore, the authors explain the importance of routines in relieving tension and allowing the use of mental capacity for more important endeavors.

Moreover, although Giddens (1984) did not discuss Berger and Luckmann's explanation of how agency is created during the first years, he does invoke Erik Erikson's analysis of the infant's psyche in an attempt to highlight the importance of routines. According to Giddens, Erikson states that an infant's first social achievement is his/her willingness to let the mother out of sight without undue anxiety. This is achieved, furthermore, once the infant acquires both an inner certainty and an outer predictability. Also, this experience, as it becomes consistent and continuous, results in a rudimentary sense of self identity. Unconsciousness comes into the discussion as it is there where the methods developed by the infant to cope with this anxiety remain (as this happens before the infant's linguistic stage). As the infant learns to deal with this anxiety, he/she develops a general state of trust and learns to rely on the sameness and continuity of outer providers (M acintosh and Scapens 1990 referring to Erikson) ${ }^{27}$. Conclusively, the primary need of ontological security affects how, later in life, agents consider predictable, stable, and ordered situations to be the way to cope with unconscious anxiety. Therefore, Macintosh and Scapens (1990) argue, ontological security explains "why agents routinely reproduce behaviours, even those which they might readily recognize as excessively coercive". Routinization provides to individuals the safety which is needed for coping with the anxieties that characterize the contexts of interaction (Busco et al. 2006, p. 15). Finally, routinization is clearly grounded on the more tacit practical type of consciousness (Giddens 1984, p. 60).

\section{On Power and Resources}

Giddens (1984, p. 9) explains that agency "refers not to the intentions people have in doing things but to their capability of doing things in the first place [...] Agency concerns events of which an individual is the perpetrator, in the sense that the individual could [...] have acted differently. Could have acted differently suggests the capability of deploying certain power in order to either intervene or refrain from such intervention" (p. 14).

Furthermore, in explaining power, Giddens (1984, p. 15) introduces the concept of resources as "structured properties of social systems, drawn upon and introduced by knowledgeable agents in the course of interaction". This definition highlights the fact that resources and structures are closely related. In fact, as it was introduced previously, structures are sets of rules and resources. Moreover, power, which is exercised through resources, presumes regularized relations of autonomy and dependence between actors during social interaction (p. 16). In addition, subordinates also have access to resources which they can use to influence their superiors. Therefore, by introducing resources into the discussion, Giddens expresses the duality of structure in power relations which he describes as the dialectic of control in social systems.

M oreover, Hardy (1996) presents a model which integrates four dimensions of power: (i) power of resources, (ii) power of processes, (iii) power of meaning, and (iv) power of the system. First, power of resources connotes a common conceptualization of power where actors use it to influence decision outcomes and specific actions. This category of power is exercised by deploying resources such as information, expertise, credibility, prestige, control of money, rewards and sanctions. Power over resources, although common, does have limitations as Hardy

\footnotetext{
${ }^{27}$ With regards to the infant's psyche, Erikson (according to Macintosh and Scapens 1990) claims that the absence of this basic trust is a fundamental cause of schizophrenia and may lead to trust anxiety complex in adulthood.
} 
(1996, p. 7) clearly explains: "continual use of 'carrot' or 'stick is required to ensure continued change; repeated use of the 'stick' may be counter-productive". Second, power of processes, also termed 'non-decision making', refers to the situations where dominant groups invoke a variety of procedures and political routines to influence outcomes by preventing subordinates from participating in decision-making. This is an interesting type of power because it may appear from 'behind the scenes' through the use of procedures and (political) routines, resulting in the most visible decision makers not being necessarily the most powerful ones. Furthermore, this type of power is relevant inside institutional theory as it may be limited if new behaviour challenges existing values and norms (i.e. institutions). Third, power of meaning occurs when symbols, rituals, or language are used to shape perceptions, cognitions and preferences in order to make change appear legitimate, desirable, rational or inevitable by giving it a new meaning. This category of power could face limitations to the degree in which behaviour (being difficult to change) is intended to be modified. Fourth, referring to power of the system, Hardy calls for the acknowledgement of the type of power which is taken for granted and, therefore, is deeply embedded within organizational systems. Once again, the relevance of this latter type of power with institutional theory is clear. Power of the system is somehow unique as it resides "often beyond the reach of tampering by organizational members [...] It is against this dimension of power that mangers must employ the other three dimensions if they are to bring about strategic action" (Hardy 1996, p. 8).

This subsection has drawn to an end after having reviewed Giddens's interpretation of agency, together with some discussions about consciousness, the role of routines in helping us cope with anxiety, and, finally, power and its relation with resources. As structures are sets of rules and resources, the following section draws on the discussions above in furthering the understanding of structures.

\section{A deeper view on Structures and its relationship with Rules, Resources, and Institutions}

This section provides further discussion on the notion of structure by exploring its definition and its relationship to depth, power and institutions. By depth I refer to Sewell (1992) states that a deep structure is one that lies beneath and generates a certain range of ordinary or "surface" structures, in a similar way in which all structures underlie and generate practices.

\section{Structures as Rules and Resources}

As mentioned above, Giddens (1984, p. 17) defines structure as rules and resources that bind space and time into social systems. Actually, structure really refers to structural properties which make it possible for discernibly similar social practices to exist in space and time and which lend them a systematic form. Moreover, structures (i.e. the plural form of structure), refer to rulesresource sets, implicated in the institutional articulation of social systems. Furthermore, Sewell (1992) notes that as structures exist as structural properties (or as the principles that pattern social practices), structures only have a virtual existence and therefore they do not exist in time and space except as the memory traces and as instantiated in action.

As structures are composed of rules and resources, it might be helpful to discuss these and, in this way, try to take distance of the abstract nature of the structure notion. Rules (of social life) are defined as "techniques or generalizable procedures applied in the enactment/reproduction of social practices" (Giddens, 1984, p. 21). In this sense, formulated rules (e.g. those given in 
canons of law or rules of game) are codifications of rules and not rules as such. Moreover, and very important for the discussion on the duality of structure that follows, rules are divided into "modes of signifying or meaning constitution and normative sanction" (Giddens, 1984, p. 28). This means that rules can be used both to provide meaning and actually sanction accordingly. For example, the rules of association football (i.e. football or soccer) are explained in the Laws of the Game published by FIFA. The Laws of the Game, for example, state that if a defender commits a foul on an opponent (or a handball) inside the penalty area, the opponent's team is awarded a penalty kick. This rule can be used to both signify (i.e. provide meaning) and sanction.

Although resources were mentioned in the previous section in explaining their relationship to power, more issues on the notion can be addressed. Resources are defined as "the media whereby transformative capacity is employed as power in the routine course of social interaction" (Giddens, 1979, p. 92). Moreover, resources can be classified in two kinds: (1) allocative resources which involve command over material objects and goods such as machines, weapons or intelligent networks; and (2) authorative resources that result of the domination of some agents over others. In addition, Sewell $(1992$, p.9) states that while authorative resources refer to human resources, allocative resources refer to nonhuman resources. While nonhuman resources include "objects, animate or inanimate, naturally occurring or manufactured, that can be used to enhance or maintain power"; human resources refer to "physical strength, dexterity, knowledge, and emotional commitments that can be used to enhance or maintain power, including knowledge of the means of gaining, retaining, controlling, and propagating either human or nonhuman resources".

Sewell (1992), however, firmly criticizes Giddens's concepts of structure (together with rules and resources) and, while warning about its elusive character, mentions how it has been nearly impossible for scholars to define the term. Sewell $(1992$, p. 6) argues that Giddens does not present any examples of rules that underlie any actual social practice but simply leaves the idea that rules are virtual and that somehow they generate social practices and systems. Furthermore, according to Sewell (1992, p.9), Giddens's concept of resources is even less adequately theorized when compared to rules.

In an attempt to construct on the definition of rules, Sewell proposes that rules exist at different levels, and, perhaps more importantly, suggests a change in terminology where the term schema is used rather than rules. Sewell $(1992$, p. 8) states that "schemas are generalizable procedures applied in the enactment/reproduction of social life". It is important to introduce the notion of schema as it is referred to by other authors whose works are relevant to this dissertation (e.g. Busco and Scapens 2011).

\section{Structures, Institutions, Depth and Power}

Furthermore, Giddens (1979) explains that it is possible to analyze how 'deeply-layered' structures are in terms of two aspects: (a) the historical duration of the practices they recursively organize, and (b) how widespread they are across a range of interactions. Institutions, Giddens's explanation continues, are the most deeply-layered practices constitutive of social systems. In this respect, it seems that in Giddens's discourse, structures and institutions are actually synonyms. Giddens (1979, p. 71) very swiftly passes from discussing structures and their relationship with the duality of structure, into affirming that institutions do not work "behind the backs" of actors as every competent member of every society knows a great deal about the institutions of that society. Moreover, given that Burns and Scapens $(2000, p .11)$ state that 
"institutions are the structural properties which comprise the taken-for-granted assumptions about the way of doing things", one could assume that institutions, as considered by Burns and Scapens, are in fact synonyms of Giddens's structures. However, this is not so, because by considering institutions as the most deeply layered practices Giddens suggests that all institutions are structures but not all structures are institutions.

Another problem arising from Giddens's relationship between structures and institutions is that the notion of depth has also been a metaphor of linguistic and structuralist discourse (Sewell, 1992). Sewell tries to construct over this obstacle by stating that a deep structure is one that lies beneath and generates a certain range of ordinary or "surface" structures, in a similar way in which all structures underlie and generate practices. For Sewell (1992, p. 27), structures "are constituted by mutually sustaining cultural schemas and sets of resources that empower and constrain social action and tend to be reproduced by that action." Therefore, power returns into the discussion and, together with depth, allows Sewell $(1992$, p. 22) to argue that the concept of structure actually includes structures of different characters ranging from those which "shape and constrain the development of world military power to those that shape and constrain the joking practices of a group of Sunday fishing buddies or the erotic practices of a single couple". Acknowledging the wide range involving structures, Sewell does not propose a typology, but opts to indicate the two dimensions that act as drivers of variance in structures: (i) depth or the schema dimension of structure; and (ii) power referring to the resource dimension.

Furthermore, Sewell argues that by thinking in terms of depth and power one can better understand the dynamics and durabilities of language, state, and capitalism, which, the author argues, are three important types of structures (see Table 6).

Table 6. Sewell's notes on depth, power, language, states, and capitalism ${ }^{28}$

\begin{tabular}{|l|l|l|}
\hline Structure & \multicolumn{1}{|c|}{ With respect to depth } & \multicolumn{1}{c|}{ With respect to power } \\
\hline Language & $\begin{array}{l}\text { High depth as intricate phonological, } \\
\text { morphological, syntactical, and } \\
\text { semantic structures underlie every } \\
\text { sentence. Sentences, moreover, are } \\
\text { aggregated into utterances in accord } \\
\text { with discursive structures of rhetoric, } \\
\text { narrative, metaphor, and logic. } \\
\begin{array}{l}\text { Furthermore, all of these structures } \\
\text { underlie the multitude of structures } \\
\text { that rely on speech and writing. }\end{array}\end{array}$ & $\begin{array}{l}\text { Low power as the structures, underlying } \\
\text { language (see adjacent quadrant), have } \\
\text { relative modest resource effects. } \\
\text { Speaking a grammatical sentence } \\
\text { creates no significant power disparities } \\
\text { but rather establishes equality among } \\
\text { members. }\end{array}$ \\
\hline States & $\begin{array}{l}\text { Low depth as it is far from clear that } \\
\text { centralized and coercive states are } \\
\text { more durable than decentralized and } \\
\text { un-coercive ones. Even the relatively } \\
\text { stable states are subject to periodic } \\
\text { structural transformations (e.g. } \\
\text { Germany from 1870 to 1950, United } \\
\text { States from 1750 to 1850) }\end{array}$ & $\begin{array}{l}\text { High power as state and political } \\
\text { structures are consciously established, } \\
\text { maintained, fought over, and argued } \\
\text { about rather than taken for granted. }\end{array}$ \\
\hline $\begin{array}{l}\text { High depth of the core structure which } \\
\text { is, paradoxically, reinforced by the } \\
\text { chronic instability or unpredictability of } \\
\text { the great amount of surface structures }\end{array}$ & $\begin{array}{l}\text { High power as following the logic of } \\
\text { capitalism increases wealth for those } \\
\text { who do so and for the capitalist } \\
\text { economy as a whole }\end{array}$ \\
\hline
\end{tabular}

${ }^{28}$ Obtained from Sewell's (1992, pp. 23-26) 
Sewell (1992) concludes that deep structural schemas have two main characteristics: (i) pervasiveness in the sense that they are present in a wide range of practices, and discourses; and (ii) relatively unconsciousness as they are taken-for-granted mental assumptions or modes of procedure that actors normally apply unaware that they are applying them.

Summarizing, this subsection, after having already discussed agency in the previous section, has discussed in a more thorough manner the concept of structure and its relationship to institutions, power, and depth. Therefore, a natural way to conclude the review on structuration theory is to review, in more detail, the complex relationship between structures and agency (i.e. the duality of structure).

\section{On the Relationship between the Duality of Structure and the Modalities of Structuration}

Giddens (1979, p. 53) invokes Marx's Grundrisse ${ }^{29}$ in an attempt to show previous efforts that had already pointed to the complex relationship between agency, structure, and systems:

[Every] social item 'that has a fixed form' appears as merely 'a vanishing moment' in the movement of society. 'The conditions and objectifications of the process', he continues, 'are themselves equally moments of it, and its only subjects are individuals, but individuals in mutual relationships, which they equally reproduce and produce anew...'

With regards to the relationship between agency and structure I would like to raise three main issues. First, it is important to note that both notions presuppose one another (as opposed to opposing one another), suggesting a dialectical relation (Giddens, 1979, p.54). Second, as Giddens states, individuals are not simply social dupes governed by independent structures, but existential beings who take reflexive actions with concern to their conduct and choices. This suggests that although structures do guide the behaviour of individuals, they are not deterministically given by nature and are potentially alterable by the agents. Third, structures should not be considered as external, but internal to the activities of individuals as properties which both constrain and enable (Giddens, 1984, p. 25). The internality to agents is conceptualized as memory traces suggesting that structures have no existence independent to the knowledge which agents have in relation to their daily interaction. However, the constraining and enabling properties of structures suggests that even if individuals are not social dupes, they are indeed affected by the structures within the social systems where they interact.

Table 7. Systems, Structures, and Agents: Definitions and Relationships

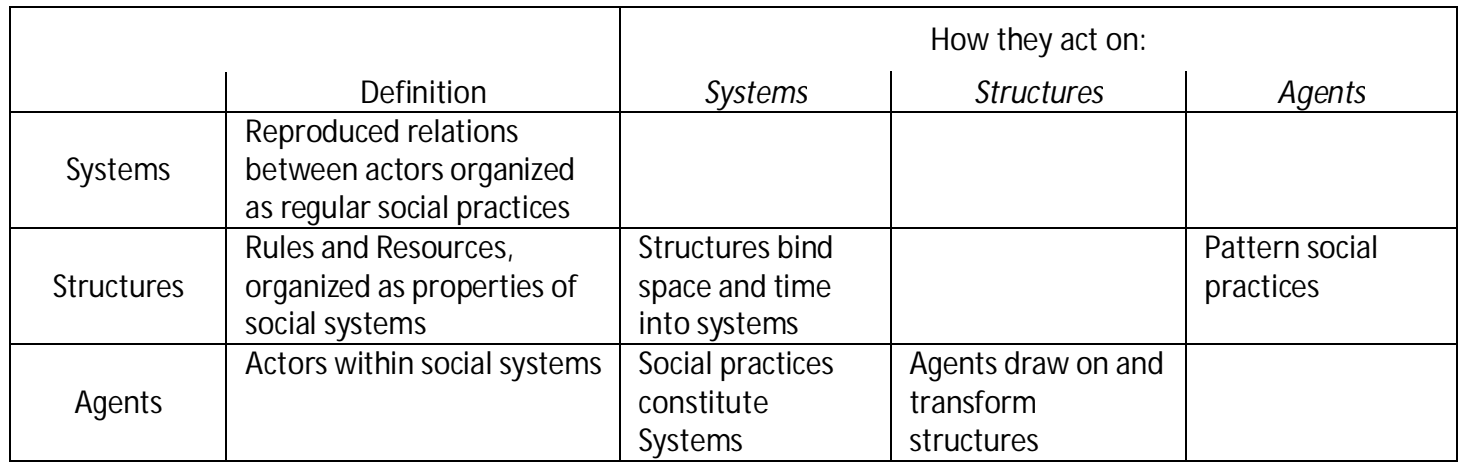

\footnotetext{
${ }^{29}$ The complete name is Grundrisse der Kritik der Politischen Ökonomie translating into Outlines of the Critique of Political Economy
} 
Table 7 serves as a summary of the definitions and relationship between systems, structures, and agents (i.e. actors or individuals). Social systems involve recurrent social practices which refer to the relations of interdependence between individuals or groups (Giddens, 1979, p 66). Social systems, furthermore, are constituted by structures (structural properties that include rules and resources) from where actors draw upon in the production of interaction. Simultaneously, those structures are reconstituted through such interaction.

\section{Giddens's M odalities of Structuration}

Giddens (1984, p. 28) introduces the modalities of structuration to provide further clarification on the duality of structure. Individuals draw on modalities in their daily activity of interaction and in the reconstitution of the structures (Busco 2009). In other words, the duality of structure does not occur between actors and the whole structure, but between actors and the modalities of the structure which is one of the three parts of the structure. The other two parts are the structure and the interaction, including the actual interaction of agents. This, perhaps, explains why structures are considered to be both external and internal to actors.

In continuing this discussion, the reader must remember that structures can be interpreted as sets of rules and resources where rules can be divided into modes of signifying and normative sanctions, and resources have an intricate relation with power. Therefore, structures serve three distinct (but interrelated) purposes: they communicate meaning, facilitate power, and sanction norms.

Giddens (1979, p. 81; 1984, p. 28), based on these three distinct (but interrelated) aspects, introduces the idea of structures having three distinct (but interrelated) dimensions: (i) signification, (ii) legitimation, and (iii) domination. While the first two represent meaning and sanction respectively (therefore rules), the latter represents power (therefore resources). In addition, each dimension is constituted by three parts: (1) structure; (2) modality; and (3) interaction ${ }^{30}$.

Furthermore, as all social practices involve the three dimensions, it is important to understand their significance and relationship. Table 8 depicts the relationship between the three dimensions and the three parts of a structure.

Table 8. The dimensions of social structure (adapted from Giddens 1979, p. 82)

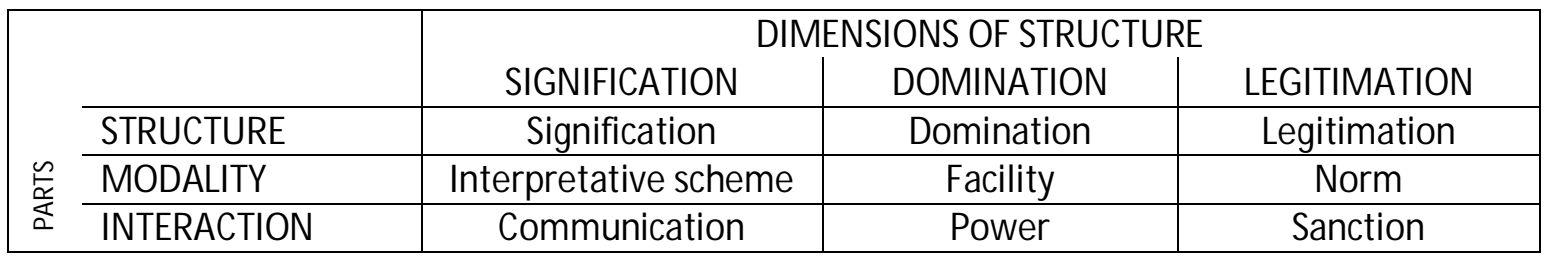

The signification dimension "is the abstract cognitive dimension of social life whereby agents communicate with each other" (Macintosh and Scapens, 1991, p. 137). Macintosh and Scapens clarify that the signification structure includes the connected and related webs of semantic code which are needed for the communication of meaning and knowledge. Furthermore, the interpretative scheme modality (i.e. the modality of the signification dimension) refers to the

\footnotetext{
${ }^{30}$ Some authors refer to each dimension with the same name as the structure inside of it (see M acintosh and Scapens, 1990) which might result in confusion.
} 
"stocks of knowledge, applied by the actors in the production of interaction [...] that form the core of the mutual knowledge whereby an accountable universe of meaning is sustained through and in processes of interaction" (Giddens, 1979, p. 83). Moreover, at the level of interaction, communication is conducted through discursive practices including speech and writing. Therefore, summarizing, the signification dimension serves agents by providing them a structure from where they can draw the semantic codes which they need to communicate meaning by accessing stocks of knowledge (i.e. interpretative schemes) which allow them to conduct discursive practices. M acintosh and Scapens (1991) refer to language to provide a good example. Language is a signification structure consisting of words and vocabulary. Agents draw on the codes of language to communicate with other agents through, for example, speech or writing. By interacting in such a way, agents reproduce language, ultimately supporting its institutionalization and persistence in social life.

The legitimation dimension provides the moral underpinnings of the signification dimension. The legitimation structure consists of legitimacy codes, normative rules and moral obligations which make up the moral constitution of social action (Macintosh and Scapens, 1991, p. 142). In other words, the legitimation structure includes the shared sets of values and ideals about, for example, what is regarded as virtue and what is regarded as vice; what counts as important and what counts as unimportant; what ought to happen, and what ought not to happen. In doing so, these values define the mutual rights and obligations that are expected from agents across a wide range of contexts of interaction. The modalities of this dimension can be divided in two: (1) norms (i.e. codes and rules) for proper conduct which serve the normative aspect of the dimension; and (2) the rule following behaviors of agents which serve the moral obligation aspect. The interaction part of the legitimation dimension refers to sanction through which morality is reproduced by allowing agents to reward and penalize other agents for complying (or not) with the codes of conduct. Summarizing, the sets of values withheld in the legitimation structure are drawn upon by agents through codes and rules which allow them to sanction other agents for their compliance (or noncompliance) with regard to the normative aspects and moral obligations embedded in a particular social system. Moreover, by recurring on these sanctions, a set of shared values can maintain their institutionalized nature in a particular system.

While the signification dimension relates to meaning, and the legitimation dimension deals with morality, the domination dimension, which has its theoretical domain in the 'theories of resource authorization and allocation' (Englund et al. 2011), centers on power. The domination structure consists of the codes for the relations and ordering of dependence and autonomy within a social institution. The modalities of this dimension refer to resources that provide the medium for domination. These resources, as explained earlier when discussing structures, are classified between allocative (e.g. raw materials, land and technology) and authorative (e.g. technical knowledge, authority, and linguistic skills) (Englund et al. 2011). Moreover, on the interaction level, power is exercised both in a broad sense, when power is considered as an ability to get things done and make a difference in the world; and in the narrow sense, when it simply implies domination (M acintosh and Scapens 1991, Busco 2009). Finally, the importance of power cannot be sufficiently underlined. Englund et al. (2011, p. 496) state that structuration theory presumes power as an integral element of social life.

Moreover, because individuals draw from the modalities of each dimension, these modalities become central to the overall discussion. Individuals employ interpretative schemes from the signification dimension/structure to communicate the mutual meanings and shared understandings. Moreover, through norms and moral codes, individuals draw legitimacy codes, 
normative rules, and moral obligations to sanction and hold accountable other individuals. Furthermore, individuals also use allocative and authorative resources to draw the rules and templates for the relations and ordering of dependence and autonomy in an organization.

\section{Quoting Giddens:}

Authorization and allocation are only mobilized in conjunction with signifying and normative elements; and, finally, legitimation necessarily involves signification as well as playing a major part in co-ordinating forms of domination. These very connections, however, make the signification-domination-legitimation scheme a useful basis for a classification of institutions which emphasizes their interrelation within the social totality. (Giddens 1979, p. 107)

The previous quote, once again, stresses that the differentiation of signification, domination and legitimation is an analytical one, not substantial one.

\section{Management Accounting and the M odalities of Structure}

Englund et al. (2011, p. 503) argue that structuration theory provides three main contributions to accounting research: (i) the introduction of a duality perspective; (ii) the conceptualization of accounting as an interwoven totality comprised of structures of signification, domination, and legitimation; and (iii) a basis for theorizing both origins of accounting continuity and change.

As noted, structures, which exist only as structural properties, can be better understood when analyzing the three dimensions that compose them (i.e. signification, legitimation, and domination), each one divided in three parts (i.e. structure, modality, and interaction). Moreover, Macintosh and Scapens (1990, p. 462) state that MASs "represent modalities of structuration in the three dimensions of signification, legitimation and domination". Hence, MASs represent the medium in which actors draw from structure in their day-to-day interaction. Simultaneously, given the duality of structure, MASs also serve as the medium for the ongoing reconstitution of these structures.

However, structures are divided into three interrelated dimensions serving different purposes. Therefore, MASs, as the modalities that they are conceived to be, also serve different purposes as explained in the following passage:

In the signification dimension, management accounting systems are the interpretative schemes which managers use to interpret past results, take actions, and make plans. In the domination dimension, management accounting systems are a facility that management at all levels can use to co-ordinate and control other participants. And in the legitimation dimension, management accounting systems communicate a set of values and ideals about what is approved and what is disapproved; justify the rights of some participants to hold others accountable; and legitimate the use of certain rewards and sanctions. (Macintosh and Scapens 1990, p. 462)

Furthermore, considering MASs as modalities suggests that they are part of the structural properties of social systems from where individuals draw for conducting their day-to-day interactions. In the signification dimension, MASs mediates between structures and agents in the form of communication - of shared rules, concepts, and theories - between the members of an organization who use these systems (Busco, 2009, p. 257). Furthermore, in the legitimation 
dimension, Busco explains, MASs are deeply implicated in the reproduction of values and serve as a medium through which the legitimation structure - embodying the legitimacy codes, normative rules and moral obligations - may be used by individuals in order to sanction (or reward) other members of an organization. M oreover, M ASs serve as resources which agents can use to draw from the domination structure - comprising the rules, codes, templates and resources drawn upon the relations of power - in the exercise of power, control, and accountability.

The depiction in Figure 9 integrates some important points of the discussions by Macintosh and Scapens $(1990,1991)$ and Busco (2009).

THE THREE DIMENSIONS OF SOCIAL STRUCTURE



Figure 9. The Three Dimensions of Social Structure and Management Accounting Systems

Hence, this discussion has highlighted the important role of MASs in structuration theory. It is important to note the important role management accounting scholars have associated with respect to cost consciousness. 
This discussion draws the exploration on structuration theory (whose relation with institutional theory has been noted) to an end. Institutions, as expressed earlier, can be regarded as standardized modes of behaviour playing a basic and fundamental part in the time-space constitution of social systems. The standardization of behaviour can, therefore, be considered as institutionalization. By the end of this section, however, when discussing the dimension of structural properties, the relationship between institutions and structuration theory became more evident. However, this relationship seems to hold no consensus, therefore resulting in a certain degree of confusion on the matter. Hence, I believe that it is not possible to agree on how Giddens's conception of structure differs and resembles the notion of institution from the perspective of various institutional branches. Finally, apart from reviewing various issues concerning structures and institutions, this chapter also highlighted important aspects with respect to agency. One of these aspects refers to the vital role of routines in our day to day interaction and as mechanisms for controlling anxiety and existential security. The following subchapter turns towards the discussion on Institutional Economics by focusing on some of the central tenets of this institutional branch, some of which have already been analyzed from a structuralist perspective in this subchapter.

\subsection{Institutional Economics: Darwin, Rules, Routines, and Institutions}

According to Barley and Tolbert (1997, p. 112) structuration theory and institutional theory provide complementary insights, not only due to a prioritization of institution/structure over action, but also because both acknowledge that institutions/structures are created, maintained and changed through action. While acknowledging the lack of consensus on the differences between institutions and structures, I will now refer mainly to institutions as this chapter deals with institutional theory.

According to Powell and DiMaggio (1991), institutional theory is paradoxical, ambiguous, and, although it allows the study of a broad range of phenomena, it is often easier to find consensus about what it is not, rather than consensus on what it is. In an attempt to reduce the paradox and ambiguity, this subchapter focuses on institutional economics which, as explained in the introduction of this chapter, has served as a sound theoretical realm for various management accounting studies. Hence, an introduction of institutional economics will be followed by a discussion on some of the central tenets of Institutional Economics by drawing on the discussions already held in this chapter. The first section will discuss the relationship between institutional theory and Darwinian Evolutionary Theory, and, furthermore, explores how rules, routines, and institutions are conceived in Institutional Economics. The second section focuses on the processes of routinization, institutionalization, and de-institutionalization which accompany the interaction of these rules, routines and institutions.

\section{Introduction to Institutional Economics}

If one wishes to explore Institutional Economics, it would be wise to begin with the American economist and sociologist Thorstein Veblen (1857-1929) whose works are commonly regarded as 
important foundations for both Evolutionary Economics and other institutional theories (Burns 2000, Johansson and Siverbo 2009, Rutherford 2001) ${ }^{31}$.

Veblen defined institutions in one of his early works:

As a matter of course, men order their lives by these principles and, practically, entertain no question of their stability and finality. That is what is meant by calling them institutions; they are settled habits of thought common to the generality of men. (Veblen 1909, p. 626)

Another important figure in Institutional Economics is Walton Hamilton who would draw on Veblen to construct his well referred definition of institution as "a way of thought and action of some prevalence and permanence, which is embedded in the habits of a group or customs of a people" (Hamilton 1932, p. 84).

Veblen's work built the foundations for institutional economics and rapidly received many adepts including John R. Commons who not only saw institutions as the necessary conditions for social improvement (Langlois, 1989) but also developed the concept of the transaction as the basic unit of analysis ${ }^{32}$ (Williamson, 1998). The interest in studying, developing, and applying institutionalist ideas into American economics rose until after the First World War (Hodgson, 1998) partly due to Veblen's criticism of neoclassical economics. Rutherford (2001) explains that the First World War had brought to light the importance of improved economic data, policy analysis, and role of government; factors that consequently provided ground for this new economic thinking that, when compared to orthodox economics, claimed to be (i) more "modern" due to its affinity to other sciences such as psychology, law and its exposure to the German historical school which had a significant impact in American economics in the late $19^{\text {th }}$ century; and (ii) more "scientific" as it was both more empirical and more in line with the latest research on other disciplines.

However, after the 1940s, various factors drove Institutional Economics to become practically marginalized in the academic arena; and, furthermore, institutions became almost a prohibited subject within mainstream economics (Rutherford, 2001). Some authors seem to consider factors which do not necessarily highlight a flaw on early Institutional Economics per se. For example, Hodgson (1998) claims that the marginalization of early institutionalism occurred for reasons including: (i) the profound shifts from instinct to behaviorist psychology and positive philosophy in social sciences between 1910 and $1940^{33}$; and (ii) the rise of a mathematical style of neoclassical economics after the Great Depression. Rutherford (2001) agrees with the first point and argues that the move towards behaviorism became an obstacle in developing institutional theories of social norms, technological change, legislative and judicial decision-making, transactions, and forms of business enterprise. Other authors, however, highlight important faults in early Institutional Economic theory. For example, Williamson (1998, p.25) states that traditional institutionalism "ran itself into the sand" partly as a result of a lack of focus. Moreover, Matthews (1986, p. 903) described the early institutional ideals as "ill-defined" because their only clear message was that "there was something seriously wrong with neoclassical economics", and nothing else.

\footnotetext{
${ }^{31}$ Even Langlois (1989), a firm critic of Veblen, noted that there is good reason to focus on Veblen as his early writings are arguable the central wellspring of traditional institutionalization thought.

${ }^{32}$ Transaction Cost Economics would later subscribe to this idea (Williamson, 1998).

${ }^{33}$ Curiously, our current understanding of consciousness has also been hampered by the appearance of behaviorist psychology according to Flanagan (1992, p. 3), and only after its fall in the 1960 psychologists renewed the study of the mind - something that is conveniently similar to the reemergence of institutionalism.
} 
The marginalization of institutionalism and its practical prohibition as a subject in main stream economics lasted until the early 1970s when certain factors led to its resurgence. Rutherford (2001) mentions at least three factors: (i) the lack of institutional content in neoclassical theory became evident when, for example, the concern for the overregulation of markets grew and brought to light the fact that mainstream economics was missing important elements of reality; (ii) many developments in contemporary economics dealt in one way or another with topics which were previously addressed in early institutional theory including redirecting economics to a more plausible theory of psychology (e.g. work in decision making, bounded rationality) and the modern work on game theory which focused on modeling given institutional situations; and (iii) the revival of pursuing topics which were in the center of institutionalist thinking such as property rights and transaction costs.

The reemergence of Institutional Economics resulted in a division in the field between Old Institutional Economics (OIE) and New Institutional Economics (NIE).

\section{On Evolutionary Theory, Rules, Routines, and Institutions}

This section focuses on some of the most important concepts related to Institutional Economics. When reviewing the birth of Institutional Economics, it was mentioned that Thorsten Veblen discussed the importance of institutions already in 1898 in an article named "Why is Economics not an Evolutionary Science?" This title suggests a very early relationship between Institutional Economics and Evolutionary Theory.

\section{On Evolutionary Theory}

The call for integrating evolutionary and institutional theories was actually furthered by Veblen (1898). Furthermore, Langlois (1989), who is actually a firm critic of old institutional Economics, identified evolution or process as one of the few consistent aspects of traditional Institutional Economics $^{34}$. However, the people who later developed American Institutionalism did not share the appeal with the Darwinian ideas which Veblen strongly supported (Johansson and Siverbo 2009, pp. 148-149). Johansson and Siverbo (p.149), for example, name three reasons for skepticism for the use of evolutionary theory in social sciences which have been commonly presented by other authors: (i) the theory's association with Herbert Spencer's 'survival of the fittest' theory, harsh welfare systems, and Social Darwinism; (ii) the theory's association with market liberalism and conservative politics; and (iii) adopting the theory is seen to limit and narrow down the view of the social world into an evolutionary and biological perspective. In responding to the first reason of skepticism, Johansson and Siverbo $(2009$, p. 149) argue that evolution "does not mean that change necessarily leads to a better or higher development, but that changes are processes influenced by hereditary traits, variation and selection". Moreover, the authors find the second reason unjust and paradoxical when considering the association with welfare systems. With regards to the third reason, the authors state that biological similes are used only when they contribute to the understanding of economic change.

The interest in Evolutionary Theory, Johansson and Siverbo (2009) argue, was raised again among Institutional Economists when Nelson and Winter published An Evolutionary Theory of

\footnotetext{
${ }^{34}$ The other consistent aspect of old institutional economics identified by Langlois is the influence of culture and institutions on individual behaviour.
} 
Economic Change. According to Nelson (1995, p. 56) (h) $^{35}$ path-dependent and lock-in-history nature of routines and institutions allows traditional Institutional Economics to be considered as an evolutionary theory as it withholds three fundamental characteristics. First, early Institutional Economics has a purpose of explaining why something is what it is at a moment in time in terms of how it got there. Second, the explanation involves both random elements which generate or renew some variation in the variables in question, and mechanisms that systematically winnow on extant variation. Third, there are inertial forces that provide continuity of what survives the winnowing. Therefore, change is an important aspect of Institutional Economics which finds support from a theory which considerably focuses on change (i.e. Evolutionary Theory).

It is important to note that evolution, path-dependency, and lock-in-history does not mean that change cannot occur, a statement which moves this discussion to the three dichotomies of change featuring in OIE literature (Burns and Scapens, 2000): (i) formal versus informal change, (ii) revolutionary versus evolutionary change, and (iii) regressive versus progressive change. While formal change occurs by conscious design, usually through the introduction of new rules and/or through the actions of a powerful individual or group; informal change occurs at a more tacit level, for example, as new routines adapt over time to changing operating conditions. Furthermore, while revolutionary change involves a fundamental disruption to existing routine sand institutions, evolutionary change is incremental with only minor disruption to existing routines and institutions. Moreover, while regressive change enforces ceremonial dominance (thereby restricting institutional change as it discriminates between human beings and preserves existing power structures), thereby restricting institutional change; progressive change displaces ceremonial behaviour by instrumental behaviour, the latter emerging from a value system which applies the best available knowledge and technology to problems and seeks to enhance relationships. Finally, within this discussion, stability and change are not mutually exclusive as there can be elements of stability within change; and change may be necessary if things are to remain stable (Scapens, 2006).

This discussion served mainly as a reminder about the relationship between Institutional Economics and Evolutionary Theory. Moreover, the types of changes discussed above will become an important part of the analysis of the institutionalization of cost consciousness. However, for now, the focus is on understanding the tenets of Institutional Economics. Therefore, the following subsection will discuss rules, the process of routinization, and routines.

\section{Rules, Routinization, and Routines}

For clarification purposes, I would like to note that when I refer to rule or norm I am considering all kinds of rules or norms unless specifically mentioned otherwise. Rules have been defined as the techniques or generalizable procedures applied in the enactment and reproduction of social practices (Giddens, 1984). However, in approaching a more institutional perspective, I would like to refer to Burns and Scapens (2000) who define rules as the formally recognized way in which 'things should be done', or the formalized statement of procedures which usually change only at certain moments or discrete intervals. Moreover, rules provide structure and coherence to the actions of groups of individuals, and, furthermore, rule based behaviour might avoid difficulties

\footnotetext{
${ }^{35}$ Burns and Scapens (2000) remind that this evolutionary nature should not imply the Panglossian argument that only the fittest survive and optimal solutions eventually emerge, but simply recognizes that processes of change are shaped by a combination of random, systematic and inertial forces which together create the context out of which, for example, new routines may emerge.
} 
and costs of undertaking assessments on every occasion related to considering available alternatives (Scapens 1994).

The actions of individuals which become regular, that is, practices which are produced and reproduced, result in order (Giddens 1979, p.56). Moreover, this reproduction is actually quite natural, as human beings are inclined toward repeating actions which result in order. This inclination results in what is referred to as routinised patterns of behaviour. Furthermore, from an institutional perspective, the process of routinization suggests a close (but not necessary) relationship between rules and routines. By repeatedly following rules, behaviour may become programmatic and based increasingly on tacit knowledge which the individual acquires through reflexive monitoring of day-to-day behaviour (Burns and Scapens 2000). Rules that become programmatic and based on tacit knowledge are considered to have gone through the process of routinization.

Programmatic rules based on tacit knowledge are described as routines or habits of a group (Burns 2000). Hodgson (1998) defines a habit as "a largely non-deliberative and self-actuating propensity to engage in a previously adopted pattern of behaviour". Nelson and Winter (1982, p. 14) argue that routine is the general term for all regular and predictable behavioral patterns (i.e. characteristics) of firms that range from well-specified technical routines for producing things, to procedures for hiring and firing, ordering new inventory, or stepping up production of items in high demand, to policies regarding investment, research and development, or advertising, and business strategies about product diversification and overseas investment. Moreover, the authors argue that there is a great deal of business behaviour which is not routine, as, for example, high level business executives do not spend too much time at the office applying the same solutions to the same problems that they were dealing with in previous years.

Furthermore, some important points should be highlighted in order to avoid an incorrect understanding regarding the relationship between rules and routines. First, in the process of routinization, previously formulated rules may become modified as a group locates mutually acceptable ways of implementing them. Second, rules may be imposed and implemented through the establishment of routines. Third, rules can emerge out of the established routines (Burns and Scapens 2000). Fourth, routines may also emerge which either have deviated from the original rules, or were never explicitly set out in the form of rules, thus providing the opportunity to formalize the established routines in a set of rules (Burns and Scapens 2000). Hence, taking these points into account, rules do not necessarily precede routines.

Before moving to a closer look on routines, I would like to highlight five important aspects relevant to the relationships between rules and routines. First, breaking a rule is often easier when compared to changing a habit, because our awareness of our own habits is often incomplete; habits have a self-actuating character, being established in subliminal areas of our nervous system (Hodgson 1998). Second, while rules are normally changed only at discrete intervals, routines have the potential to be in a cumulative process of change as they continue to be reproduced; however, the extent of this process of change for specific routines depends on other rules and routines (Burns and Scapens 2000). Third, the relation between rules and routines at a given point/limited-period can be very loose (Lukka 2007). Fourth, a change in routines may result not only from a change in rules, but also from many other factors including experiences with existing working methods or social pressures to change. The latter could result from internal or external dissatisfaction with certain practices and performances, newly developing values in society, increasing relative power of a certain group, or other changes in internal or external institutions (ter Bogt 2008). 


\section{Routines: Characteristics and Benefits}

Routines hold a center stage in institutional literature as they contribute in explaining organizational and economic change (Becker 2004). In an attempt to organize the ideas on organizational routines, Becker states that routines have eight main characteristics. First, routines are patterns containing action, activity, behaviour, and interaction. Second, recurrence is a key characteristic of routines as, Becker $(2004$, p. 646) states, "one would be hard pressed to call something happening only once a routine". Third, routines are collective phenomena as they involve multiple actors and therefore, perhaps, involve different locations across space or across a particular organization. Ter Bogt (2008) explains that even if routines seem to be difficult to change, when an organization or its environment experience a sudden shock (e.g. government organization is going to be privatized), certain routines can go through major changes in a short period of time, perhaps because employees understand that the organization's continuity may be at stake. Fourth, routines may be characterized either by mindlessness or, to the contrary, as effortful accomplishments. Fifth, routines are processual in nature, a characteristic which allows realizing the potential contribution of the concept of routines in explaining organizational change (Becker 2004, p. 649). Sixth, routines are embedded in an organization and its structures (i.e. institutions), and are specific to the context. The latter part of this characteristic suggests that incompatibility may arise if a routine is transferred into a new context. Seventh, routines change in a path-dependent matter and are shaped by history. This suggests that the starting point matters. Finally, eighth, routines are triggered either in an actor-related or external-cued manner.

Moreover, Becker (2004, pp. 654-661) also explains why routines are important for organizations. First, routines coordinate and control. Second, routines allow truce between individuals by providing them the luxury of not having to explain or account for every single action that they make. Third, routines economize the limited cognitive resources. This effect is very important as neither all alternatives, nor all consequences of any alternative can be known so attention has to be allocated selectively. Fourth, routines reduce uncertainty as they help actors in their ability to choose. Fifth, routines provide stability, a benefit which has been grounded on two different arguments: (i) as long as an existing routing gives satisfactory results, there is no need to find another one; and (ii) for reducing costs as changing the mode of executing a specific task will most likely incur in costs. Lastly, sixth, routines store knowledge by serving as organizational memory.

\section{Routines: Conceptual Discrepancies and Solutions}

The understanding of the concept of routine, however, is also subject to discussion. Also with respect to conceptual confusion, van der Steen (2011) argues that "the lack of conceptual clarity and limited detail in the definition of routines give rise to confusion in our understanding of the way in which routines change". Van der Steen states that the extensive discussion on organizational change has led to three understandings of routines:

1. Routines can be behavioral regularities and are regarded as collective, behavioral patterns

2. Routines can be conceptualized as formal rules, such as standard operating procedures

3. Routines are viewed as dispositions, rather than behaviours or rules 
In fact, the definition of routines by Burns and Scapens (2000) as the way in which 'things are actually done' has been targeted. Englund and Gerdin (2008) argue that, on the one hand, Burns and Scapens conceive routines as theoretical constructs (not empirical categories) which facilitate an interpretation of the actions which can be observed. On the other hand, the authors claim that Burns and Scapens also suggest that routines represent empirically observables practices as such. The authors explain that while the former interpretation relates to the idea of modalities as suggested by Giddens (1984, p. 28), the latter relates to the idea of scripts suggested by Barley (1986. p. 82) and Barley and Tolbert (1997, p. 98). It might be worth noting that the Burns and Scapens Framework draws heavily from the framework presented by Barley and Tolbert (1997).

Moreover, Englund and Gerdin argue that mixing these two interpretations results in two main theoretical consequences: (1) the problem of conflation; and (2) the problem of erroneous conclusions about structural change/stability. The problem of conflation refers to the problem of reducing social structure to social action (and vice versa) which arises from defining rules and routines as modalities and as 'observable, recurrent action'. This problem, furthermore, contradicts one of the core assumptions of Giddens's structuration theory: recurrent actions are not social structures as social structures generate action (this is what is referred to as the duality of structure). The second problem, erroneous conclusions about structural change/stability, refers to the risk of drawing erroneous conclusions in empirical research as the confusion may lead to the changes in scripts to be erroneously interpreted as evidence of structural change (and vice versa) when this is not necessarily the case.

The conceptual discrepancies have also been noted by Feldman and Pentland (2003) who propose a solution by differentiating two distinct aspects of routines: the ostensive and the performative aspects. According to Feldman and Pentland (2003, p. 101), while the ostensive aspect is the idea; the performative aspect is the enactment. The ostensive aspect of a routine shapes our perception of what the routine is and may be codified as a standard operating procedure (i.e. an artifact), or it may exist as a taken-for-granted norm. Moreover, the ostensive aspect may have a significant tacit component embedded in procedural knowledge where artifacts of this aspect may exist in various forms. The authors warn not to conceptualize the ostensive aspect of a routine as a single, unified object (e.g. a standard operating procedure) as this aspect also incorporates the subjective understandings of diverse participants. Furthermore, the performative aspect of a routine is the actual performance of the routine which is best understood as inherently improvisational. In addition, the ostensive aspect acts on the performative aspect (and vice versa). On the one hand, the ostensive aspect acts on the performative by guiding, accounting and referring. This means that people may use the ostensive aspect as a guide to what actions ought to be taken, as a guide for actions already taken, or to refer to what is similar about a set of performances and thereby create routines (Feldman and Pentland 2003, p. 105). On the other hand, the performative aspect of routines is "essential for the creation, maintenance, and modification of the ostensive aspect" (Feldman and Pentland, 2003, p. 107).

Drawing on the work by Feldman and Pentland (2003), van der Steen (2011) proposes a framework for studying the processes of change within accounting routines (Figure 10). 


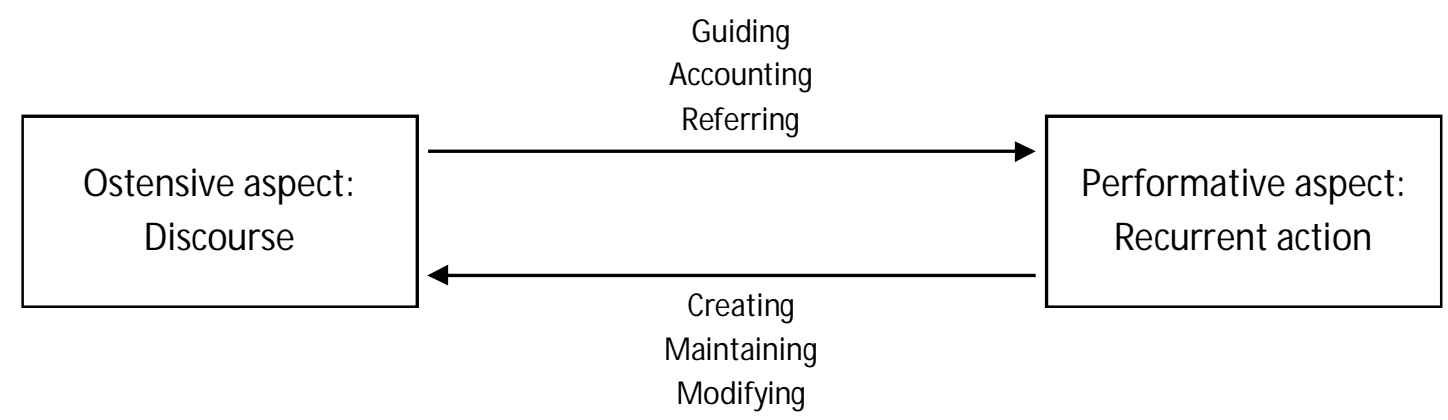

Figure 10. Routines on ongoing systems of interaction (van der Steen 2011)

The framework above highlights the recurrent relation between the two aspects depicting "routines as reproductions of ongoing systems of interaction" (Feldman and Pentland 2003, p. 516). The ostensive aspect (e.g. an artifact specifying the routines) guides, accounts and/or refers the performative aspect (i.e. the actual enactment of the routine) which, consequently, creates, maintains and/or modifies the ostensive aspect. It is important to note that improvisation is never precluded even if the ostensive aspect does limit the capability of actors to improvise.

The concepts discussed in this subsection somehow distant themselves from their use in ordinary language. This argument holds true particularly in the case of routines where it connotes repetition and stability in many languages (e.g. English, Spanish, Italian, Portuguese). For example, the sentences "a monthly verification of the budget has become a routine" or "la verificación mensual del presupuesto se ha convertido en rutina", respectively in English and Spanish, suggest that a particular behavior (in this case the periodical verification of budget) has become a repetitive and perhaps even taken for granted. This taken-for-grantedness, however, is more closely related, according to institutional literature, to the concept of institution.

\section{On Institutionalization, De-Institutionalization, Institutions and Organizational Culture}

After discussing on rules, routinization, and routines; this review has finally arrived to the concept of institution, which is, clearly, central to institutional theory. I will first describe very quickly the process of institutionalization before analyzing what an institution connotes and its relationship with organizational culture.

\section{The Institutionalization Process}

Routines can potentially experience a transformation which is analogous to the routinization process of rules. Routines, over time, can become increasingly underpinned by tacit knowledge which individuals acquire through reflexive monitoring of past behaviour, and once they become widely accepted in the organization, routines become institutionalized (Burns 2000). M oreover, a routine which has been institutionalized takes a normative and factual quality obscuring their relationship with particular interests as they are now disassociated from historical circumstances (Siti-Nabiha and Scapens 2005, p. 46).

Furthermore, Siti-Nabiha and Scapens (2005, pp. 46-47) state that "when choices or solutions to problems continue to work over time, they come to be applied in a rule-like way, and subsequently become a routine activity [...] Eventually, such routine behavior can become 
unconsciously taken-from granted; i.e. institutionalized". Therefore, one could illustrate this casual relationship as it appears in Figure 11.

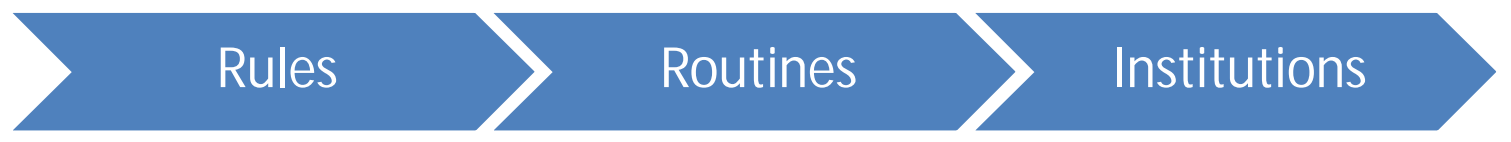

Routinization

Institutionalization

Figure 11. Casual view on Rules, Routines, Institutions, Routinization, and Institutionalization.

I would like to warn the reader not to take this casual view too strictly as the previous subsection mentioned many ways in which rules and routines do not follow a strict deterministic process. In a similar way as the relationship between rules, routinization, and routines is not a one-waystreet, the relationship between routines, routinization, and institutions does not follow such order. For example, the emergence of new routines will be influenced both by the encoding of ongoing institutions and the reproduction of existing routines (Burns and Scapens 2000).

Furthermore, it is important to note that not all behavioral patterns are institutionalized to the same extent as there may be variations in the length of time that particular institutions have been in place, and, ,moreover, in how widely institutions are accepted by the members of a collectivity (Burns and Scapens 2000). This acknowledgement, hence, shifts the attention towards the concept of institution.

Before discussing institutions, however, I would like to mention the notion of deinstitutionalization, which, as its name suggests, may be considered as the opposite process of institutionalization - 'removal' or 'reversal' of a previously established institution. Deinstitutionalization refers to the discontinuity of an institutionalized organizational activity or practice (Oliver 1992) ${ }^{36}$. However, in contrast to the vast literature on institutionalization of practices, not many efforts have been devoted to the study of de-institutionalization (Powell and DiM aggio 1991, Davis et al. 1994, Ahmadjian and Robinson 2001) or similar concepts such as strategy abandonment (Greve 1995). M oreover, Davis et al. (1994) argue that empirical work on institutionalization offer little sense of the mechanisms responsible for de-institutionalization implying that institutionalization is a once-and-for-all process. However, as Ahmadjian and Robinson (2001) explain, new practices, often, cannot be adopted unless previously established ones are removed; and, furthermore, understanding the processes of erosion of institutionalized practices may allow a better understanding of change, a central factor in institutionalization (and de-institutionalization) theory.

Institutions: Definitions and Characteristics

Douglas states:

A convention is institutionalized when, in reply to the question, "Why do you do it like this?" [...] the final answer refers to the way the planets are fixed in the sky or the way that plants or humans or animals naturally behave (Douglas 1986 p. 46-47)

\footnotetext{
${ }^{36}$ De-institutionalization is also considered as "a policy intended to decrease reliance on large, segregative, exclusionary institutions in particular state and county mental hospitals and support the development of a network of treatment programs and caregivers located in a community" (Wright et al., 2000).
} 
The statement by Douglas is picturesque but, simultaneously, provides a sense of the difficulties in defining the notion of an institution. These difficulties have been addressed by other scholars. For example, Scott (1987) states that the "concepts of institution and institutionalization have been defined in diverse ways, with substantial variation among approaches. Thus, the beginning of wisdom in approaching institutional theory is to recognize at the outset that there is not one but several variants". Moreover, Goodin $(1998$, p.21) concurs with Scott by stating the following:

There is wide diversity within and across disciplines in what they construe as "institutions" and why. That diversity derives, in large measure, from the inclination within each tradition to look for definitions that are somehow "internal" to the practices they describe [...] When we are looking at contract law or congressional commutes or whatever, what "the" institution is (and hence, to some extent, what "an" institution is in that setting) is traditionally tied up with what it does and why it is there. And since explanandums vary across disciplines, so do explanations - and hence so too do definitions of the very notion of an "institution" itself.

Institutions, however, have been defined in various forms and I believe that the proper way to address them is to simply present them as they appear in Table 9.

Table 9. Definitions of institutions identified

\begin{tabular}{|l|l|}
\hline \multicolumn{1}{|c|}{ Definition of institutions } & \multicolumn{1}{|c|}{ Reference } \\
\hline Settled habits of thought common to the generality of men & Veblen (1909, p. 626) \\
\hline $\begin{array}{l}\text { A way of thought and action of some prevalence and permanence, } \\
\text { which is embedded in the habits of a group or the customs of a } \\
\text { people }\end{array}$ & Hamilton (1932, p.84) \\
\hline A stable, valued, recurring pattern of behavior & Huntington (1968, p. 12) \\
\hline $\begin{array}{l}\text { Standardized modes of behaviour which play a basic part in the } \\
\text { time-space constitution of social systems }\end{array}$ & Giddens (1979, p. 96) \\
\hline The more enduring features of social life & Giddens (1984, p. 24) \\
\hline $\begin{array}{l}\text { The rules of the game in a society or, more formally, the humanly } \\
\text { devised constraints that shape human interaction }\end{array}$ & North (1990, p. 3) \\
\hline $\begin{array}{l}\text { Shared rules and typifications that identify categories of human } \\
\text { actors and their appropriate activities and relationships }\end{array}$ & Barley and Tolbert (1997, p. 96) \\
\hline $\begin{array}{l}\text { The shared taken-for-granted assumptions which identify categories } \\
\text { of human actors and their appropriate activities and relationships }\end{array}$ & Burns and Scapens (2000, p. 7) \\
\hline The way things are & Burns and Scapens (2000, p. 7) \\
\hline $\begin{array}{l}\text { The structural properties which define the relations between, and } \\
\text { the activities of, the members of particular social groups or } \\
\text { communities }\end{array}$ & Burns and Scapens (2000, p. 8) \\
\hline The unquestioned way of doing things & \\
\hline
\end{tabular}

It is important to remind that these definitions of institutions do not refer necessarily to the same concept. For example, as I have already stated, Barley and Tolbert (1997, p. 96) argue that their notion of institution has a strong resemblance to Giddens's notion of structure and Sewell's (1992) idea of a schema. However, Burns and Scapens (2000, p. 7) state that Barley and Tolbert's definition of institution "contains both systems (thoughts and actions of some prevalence and permanence) and structure (embeddedness in habits and customs)". Moreover, Sewell did not treat schema and structure as the same notion, but, instead, used Giddens's definition of rules to denote schema. In addition, the understanding of Burns and Scapens with respect to institutions is certainly very similar to Giddens's conceptualization of structures. Burns and Scapens (2000, 
pp. 8-11) argue that institutions (i) act as structural properties; (ii) exist only in the understandings and stocks of knowledge of individuals or groups - as they (institutions) are disassociated from their particular historical circumstances; and (iii)bind time by shaping actions period-by-period.

Furthermore, both Hogdson (1998) and Guerreiro et al. (2006, pp. 199-200) discuss the characteristics which all institutions share at some degree. While Hogdson names five, Guerreiro et al. identify six. I have synthesized them into five:

1. Institutions are based on shared conceptions and expectations which are taken-forgranted. This is what Guerreiro et al. consider as the assumed nature of institutions.

2. Institutions are intricately related to routines. In some instances, routines become part of the institutional realm through the process of institutionalization. Moreover, as already mentioned, the emergence of new routines will be influenced both by the ongoing institutions and the reproduction of existing routines.

3. Institutions are stable. This does not mean that they are immutable or immortal, but that they are relatively durable, self-reinforcing, and persistent.

4. Institutions are collective in nature. All institutions involve a complex interaction of agents analogous to Giddens's duality of structure

5. Institutions provide meaning, legitimation, and power. Analogously to Giddens's dimensions of structures, institutions allow actors to communicate meaning, sanction norms, and facilitate power.

Finally, simply as an illustrative example which will allow better understanding of the following section, I would like to present the following passage from Barley and Tolbert (1997):

[Institutions] are to social action as grammars are to speech. Speech allows for an infinite variety of expressions, yet to be comprehensible, every expression must conform to an underlying set of tacitly understood rules that specify relations between classes of lexemes. Similarly, social actions may vary in their particulars, but to be interpretable, their contours must conform to taken-for-granted assumptions about the activities and interactions appropriate for different classes of actors. The specifics of any grammar or institution are arbitrary in the sense that both are essentially social stipulations. Adherence to such stipulations is far from arbitrary, if by arbitrary one means entirely open to individual choice. (Barley and Tolbert, 1997, p. 96)

Although I find this analogy useful, it is important to note that some authors, including Sewell (1992, p. 23), warn about the tendency to use linguistic structures as paradigms for structures in general.

\section{Organizational Culture and Institutions}

The introduction of this dissertation presented the quote by Dekker $(2004$, p. 37) which suggests that cost consciousness could be considered as a type of business culture. However, given the understanding of cost consciousness in 2004, it is very plausible that Dekker could have opted to use other notions instead of cost consciousness. Nevertheless, one could say that Dekker's subconscious allowed a platform for pondering on cost consciousness's possible role in an organizational culture. 
Clifford Geertz, a well cited scholar with respect to organizational culture (Ouchi and Wilkins, 1985), defined culture as "the fabric of meaning in terms of which human beings interpret their experience and guide their action" (Geertz 1993). Furthermore, Schein (2004, p. 17) provides the following definition of culture:

A pattern of shared basic assumptions that was learned by a group as it solved its problems of external adaptation and internal integration, that has worked well enough to be considered valid and, therefore, to be taught to new members as the correct way to perceive, think, and feel in relation to those problems

Moreover, Schein $(2004$, p. 26) has introduced the Levels of Culture which allows the analysis of culture at three levels (levels denoting the degree to which a cultural phenomenon is visible to observers). On the one hand, the deepest level of culture (i.e. the less visible to observers) refers to the basic assumptions concerning unconscious, taken-for-granted beliefs, perceptions, thoughts, and feelings. On the other hand, the most superficial manifestation of culture refers to artifacts which can take the form of stories, myths, metaphors, rites, rituals, ceremonies, heroes, and symbols (Brown 2011, p.12). In addition, between these two levels exists an intermediary level composed by espoused beliefs and values which include philosophies. Finally, with respect to a general description on the Levels of Culture, the understanding of a group's culture requires getting at its shared basic assumptions (Schein 2004, p. 36) as this understanding is necessary in order to interpret or decipher correctly the other levels (i.e. artifacts, beliefs, and values).

Furthermore, with respect to organizations per se, an organizational culture is also composed of basic assumptions (the deepest level of culture), espoused beliefs and values (in an intermediate level of culture), and artifacts. While values are intimately connected with moral and ethical codes determining what people think ought to be done, beliefs concern what people think is and is not true (Brown 2011, p.26). An example of belief in an organization would be the belief that increasing $R \& D$ spending would be reflected in increased revenue. Moreover, notions about what is important in an organization can vary greatly across companies; while in some settings people may care deeply about profit, in other settings people might be more interested about technology or employee well-being (Kotter and Heskett 1992, p. 4).

Moreover, Schein (2004, p. 28) explains that if a manager convinces a group to act on his/her belief given, for example, an specific target, and the group has a shared perception of that target, then the perceived perception can become transformed first into a shared value/belief, and ultimately into a shared assumption. However, Schein warns that all beliefs and values do not undergo such transformation. Hence, the relationship and possible transformation between espoused beliefs/values and assumptions are analogous to the relationship between routines and institutions. The reader might recall that some routines can undergo a process of institutionalization and become part of the institutional realm. However, as explained, this does not always occur.

Furthermore, according to Brown (2001), basic assumptions differ from beliefs in three ways: (i) while beliefs are held consciously and are relatively easy to detect, basic assumptions are held unconsciously and, therefore, difficult to notice; (ii) beliefs, as opposed to assumptions, are easier to modify as they are confrontable and debatable; and (iii) while beliefs are simple cognitions when compared to assumptions, the latter is pre-conscious, non-confrontable and highly complex aspects of human psychology. 
Moreover, I would like to refer to Schein's model for understanding organizational culture change (Schein 2004, p. 292) which identifies various mechanisms driving change as such. Schein suggests that during the early growth years of an organization, cultural change mechanisms include incremental change through evolution (e.g. small increments assimilating what works during years), insight (e.g. collectively examining and the culture), and promotion of hybrids within the culture (e.g. by promoting insiders who can adapt to external realities). Moreover, during an organization's midlife stage, change mechanisms include systematic promotion from selected subcultures (e.g. promote employees with different subcultures), technological seduction (e.g. introduction of new technologies to seduce members into new behaviours), and infusion of outsiders (e.g. hiring a new CEO). Finally, during an organization's maturity and decline, change mechanisms include scandal and explosion of myths (e.g. events which highlight incongruities between espoused beliefs and actual practices), turnarounds (e.g. by a collective involvement in highlighting incongruities of the old culture), mergers and acquisitions (e.g. the culture of one organization dominates the other one), and destruction and rebirth. In addition, the author argues that when a culture is stabilized in an organization, cultural change requires more time and effort when compared to a culture of an organization in its growth stages.

Interestingly, the quote by Dekker (2004, p. 37) - presented in the introduction - suggested that an alliance (i.e. merger) was seen as a possible driver of cultural change into cost consciousness.

Finally, I would like to highlight some important similarities between culture and the concept of institution ${ }^{37}$. First, Schein (p. 22) states that the shared basic assumptions at the core of culture have been taken for granted by the members of the group carrying that culture. Therefore, culture and institution seem to be very similar concepts as they both connote shared basic assumptions embedded in a group. Second, Schein (2004, p. 31) explains that basic assumptions are non-confrontable, non-debatable and, hence, extremely difficult to change. Furthermore, Kotter and Heskett (1992, p. 4) explain that shared values are very difficult to change as individuals may be unaware of the values that bind them together. Therefore, basic assumptions are similar to institutions as both are stable and difficult to change. Third, Schein (2004, p. 32) states that basic assumptions allow a group to function. In addition, any challenge or questioning of a basic assumption releases anxiety and defensiveness which also explains why it is not easy to change a group's culture. Thus, this characteristic of basic assumptions is similar to the discussion on the roles of routines in a person. In addition, this characteristic also resembles the collective nature of institutions. Hence, basic assumptions also share characteristics with routines. Finally, the similarity between culture and institution has been noted by Siti-Nabiha and Scapens (2005, p. 48) who state that "culture is a concept with a wired range of meanings and we prefer the term institutions". Therefore, one could reasonably argue that the notions of culture and institutions, as discussed in this dissertation, are inter-exchangeable to a certain extent.

However, I want to abstain myself from equating these two terms in order to avoid unnecessary and potentially flawed conceptual overlaps. Various authors have identified organizational institutions such as results orientation, production orientation, and financial orientation. These, although are indeed arguably broad, seem to be more like features of an organizational culture because one could reasonably imagine an organization whose culture is both product oriented

\footnotetext{
${ }^{37}$ As the reader may remember, I integrated the extant literature to formulate five characteristics which all organizations should share (i.e. institutions are based on shared conceptions and expectations which are taken-for-granted; institutions are intricately related to routines; institutions are stable; institutions are collective in nature; and institutions provide meaning, legitimation, and power)
} 
and results oriented. Hence, this dissertation takes the view of organizational culture as being composed of various institutions relationship as depicted in Figure 12.

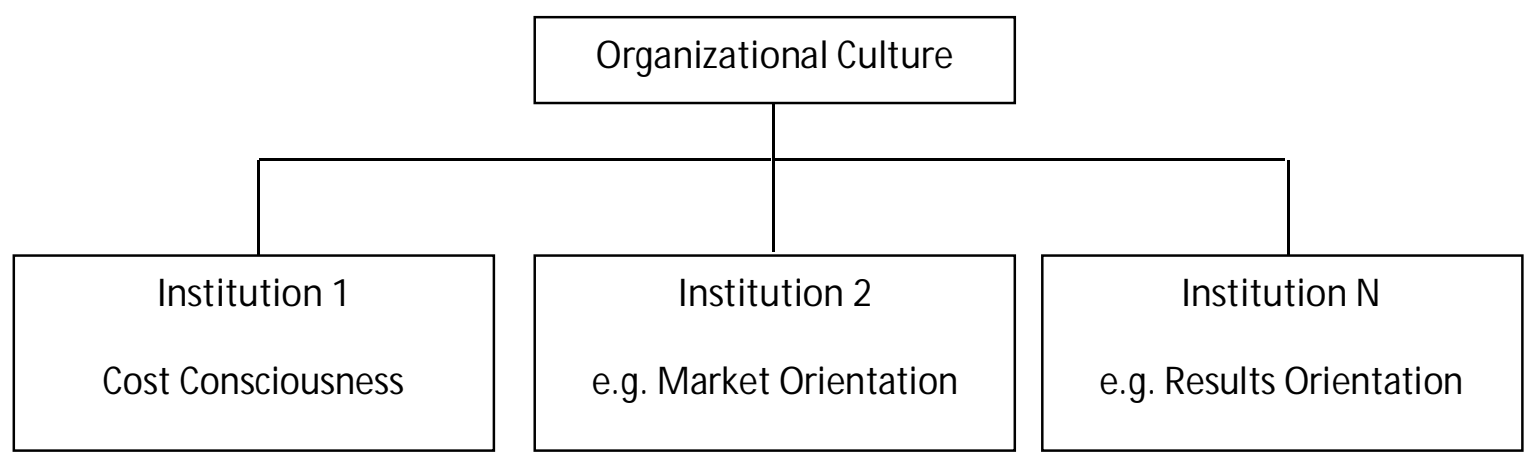

Figure 12. Relationship between cost consciousness, institutions, and organizational culture

In sum, this subchapter has reviewed the central tenets of Institutional Economics. First, a brief review on the relationship with Darwinian Evolutionary Theory was explored. The relationship, I argue, is reflected when considering how institutions are conceived as very stable, yet changeable either in a very gradual manner or as a result of a very abrupt event. Moreover, this subchapter also reviewed the concepts of rules and routines from an institutional perspective. Particularly, the latter have gathered plenty of attention since the publication of Nelson and Winter (1982). Furthermore, discrepancies with respect to the conceptualization of routines have allowed discussions on ways in which these can be better analyzed. The differentiation between the ostensive and the performative aspect of routines was highlighted as one solution for the problem of conflation related to the concept of routines. In addition, the processes of routinization, institutionalization, and de-institutionalization were reviewed where it was noted the lack of attention from scholars with respect to the latter. Finally, this subchapter also reviewed the notion of institution and its relationship with organizational culture.

One of the aims of this subchapter has been to allow a smoother overview on OIE and NIE, as it is conducted in the following subchapter.

\subsection{Old Institutional Economics and New Institutional Economics}

Institutional Economics reemerged on the 1970s with a division of the theory between Old Institutional Economics (OIE) and New Institutional Economics (NIE) ${ }^{38}$. At plain sight, this division suggests that while OIE academics maintain a firmer grip on early institutional ideas, NIE scholars have somehow diverged by embracing other values which, in certain cases, sharply oppose those exposed by the early institutionalist thinkers. This assumption, although somewhat accurate, deserves more attention. Hence, this subchapter discusses the similarities and differences between OIE and NIE.

Before this discussion, I would like to summarize some of the main ideas concerning traditional institutionalism (i.e. OIE) which have been discussed so far. OIE is concerned about rules, habits, routines and institutions; and, furthermore, moves from general ideas concerning human agency, institutions, and the evolutionary nature of economic processes to specific ideas and

\footnotetext{
${ }^{38}$ I have opted only until now to use the abbreviations as New Institutional Economics had not really been brought to light until this point.
} 
theories, related to specific economic institutions or types of economy (Hodgson 1998). Moreover, OIE thinking was born in an attempt to reconstruct economic theory and replace neoclassical economics by giving special importance to institutions, rejecting the neoclassical tenet of a taken-for-granted rational and utility-maximizing individual, and exploring how the habits of these not-identical individuals both reinforce and are reinforced by institutions.

This subchapter is divided into three main sections. The first section reviews some of the most important differences between OIE and NIE which scholars on both branches have noted during the years. The second section provides an overview on NIE to infer its suitability for the objectives of this dissertation. Finally, the third section brings New Institutional Sociology (NIS) into the conversation as it represents an institutional branch which supports this study's contributions.

\section{Main Points of Dispute between OIE and NIE}

The natural question which arises in this chapter is: what are the differences between OIE and NIE? Although there seems to be no unanimity in explaining the differences between the two institutional divergences (Hogdson 1998), it is possible to rescue some common points of dispute. Powell and DiMaggio (1991, p. 13) discuss eight main differences between old and new institutionalism in the sociological tradition including their initial focus, conceptualization of the environment, the view on organizations, and the conception of the cultural (or cognitive) bases of institutional behavior. Although some of these differences also apply in the economic branch of institutional theory, the literature discussing the differences between OIE and NIE tend to focus on the following three points:

1. NIE scholars claim that OIE lacks economic theory

2. OIE scholars claim that NIE embraces rational actor models

3. OIE's holism versus NIE's individualism

I will now provide a brief review on each of these points. It is important to highlight the importance of this section as the discussion below should provide clues for identifying the institutional branch which could provide better support to the second contribution of this dissertation (i.e. a theoretical exercise focused on understanding the elements and mechanisms involved in the institutionalization of cost consciousness).

\section{Is Old institutional theory Anti-Theoretical?}

Matthews (1986) describes the OIE doctrines as ill-defined and then states that NIE has two propositions which explain why it is now one of economics' liveliest areas: (i) institutions matter and (ii) the determinants of institutions are susceptible to economic analysis by the tools of economic theory. Given that even new institutional economists seem to agree that OIE also concurs with the first proposition, one can reasonably infer that the second proposition is the main driver of the divergence between the two institutional economic branches. 
Moreover, Ronald Coase, one of the great exponents of NIE, is quite explicit:

John R. Commons, Wesley Mitchell, and those associated with them were men of great intellectual stature, but they were anti-theoretical, and without a theory to bind together their collection of facts, they had very little that they were able to pass on. - (Coase 1998, p. 72)

The view of OIE as anti-theoretical is common among new institutional economists but is challenged by scholars who claim that characterizing OIE as purely descriptive or anti-theoretical is a misconception for various reasons. Hodgson (1998), for example, explains that even the early institutionalists like Veblen and Commons placed strong emphasis on the importance of explaining and developing economic theory along Darwinian lines which relates to Evolutionary Theory. However, in response to this argument, Langlois (1989) explains that the Darwinian theory in the early $20^{\text {th }}$ century, like Veblen, undermined issues like consciousness, thus guiding OIE into failure because "a programme that would reduce social science to 'objective' facts and observable relationships is unlikely to succeed when the 'facts' are themselves necessarily the product of human cognition". Another reason for dismissing the claim of OIE's dismissal of theory is that important empirical and theoretical work on areas like pricing was conducted by early institutionalist (Hodgson 1998, Rutherford 2001). In addition, some OIE theories, like the theory of the consumption function by James Duesenberry, were neglected not because it failed empirically but because it did not conform to the presumptions of rational choice theory (Hodgson 1998). I would like to note that the arguments in favor of considering OIE as nontheoretical tend to focus on OIE in the early $20^{\text {th }}$ century and, to my knowledge, do not address this problem on the contemporary OIE scholars.

\section{Rational Individuals}

The second point of disagreement relates to rational individuals. Hogdson (1998) argues that NIE assumes an initial institution-free state of nature and attempts to explain the emergence of institutions by reference to a model of rational individual behaviour. With this argument, Hogdson seems to want to tie NIE directly with the same kind of rational taken-for-granted utility-maximizing agent which neoclassical economics supposes. Moreover, on this respect, Douglas (1986, p. 9) argues:

It is axiomatic that the theory [of individual rational choice] that rational behavior is based on self-regarding motives. The individual calculates what is in his best interest and acts accordingly. This is the basis of the theory on which economic analysis and political theory are based, and yet we get the contrary impression. Our intuition is that individuals do contribute to the public good generously, even unhesitatingly, without obvious self-serving. Whittling down the meaning of self-serving behavior until every possible disinterested motive is included merely makes the theory vacuous.

A counter-argument from NIE supporters is that a close link between NIE to neoclassical economics is a misconception not only because NIE insists that policy analysis be guided by comparative institutional analysis and not by comparing real-world outcomes with the hypothetical benchmark of perfectly competitive general equilibrium (a neoclassical tenant) (Klein, 1998), but also because NIE does not necessarily assume neoclassical rationality but bounded rationality - a behaviour that is intendedly rational but only limited so (Williamson 
1998). Williamson (2000) explains: "there is close to unanimity within the NIE on the idea of limited cognitive competence - often referred to as bounded rationality". Nonetheless, Scapens (2006) still expresses his worry about explanations of managerial behaviour grounded in bounded rationality and opportunism, and particularly about the notion of self interest with guile as such behaviour could be considered to be behind recent economic collapses where some individuals seek to maximize their own interest, and use guile to circumvent the rules which normally govern accepted business behaviour ${ }^{39}$.

Holism (OIE) versus Individualism (NIE)

Previously, when discussing the reasons for the fall of institutionalism after the interwar period, I mentioned that Langlois (1989) considers methodological holism as one of the main inconsistencies in OIE. Moreover, Powell and DiMaggio $(1991$, p. 2) state that the older lineages of institutionalism came into disfavor by the new institutionalist thinkers "not because they asked the wrong questions, but because they provided answers that were either largely descriptive and historically specific or so abstract as to lack explanatory punch".

However, Hogdson (1998) argues that there are also clear inconsistencies in the individualism approach which considers macroeconomic phenomena as necessarily explained by microeconomic phenomena. Hodgson argues that methodological individualism is a theoretical misconception built upon an antiquated 300 year old Enlightenment-era assumption that considers taken-for-granted individuals as the building blocks of economic theory, therefore assuming an initial institution free "state of nature".

Moreover, Scapens (2006) argues that NIE's strong focus on economic factors does not allow the understanding of certain aspects of the mish-mash, or complex interplay of the disparate set of influences and the intentional actions of organization members, which need to be understood if one wishes to analyze certain processes of change, for example in management accounting.

In response against those who assault the selfish individualism, Selznick (1996) writes:

In this version of "methodological individualism" which I have long accepted, one does not slight the importance or the reality of distinctively social phenomena, such as group morale, or patterns of institutional adaptation and persistence. We do say, however, that these phenomena are produced in and through the responsive and problem-solving behaviour of individuals. This behavior does not necessarily conform to rational-actor models, but it very often does include attention to shortterm opportunities, constraints, and incentives.

Supporting this passage, Klein (1998) argues that new institutionalism eschews the holism of traditional institutionalism by following strict methodological individualism and taking phenomena like corporate culture and organizational memory as explananda and not as explanans. Furthermore, Järvenpää (2007, p. 105) states that "old institutional economics (OIE) theory, in particular [with respect to institutional theoretical works in management accounting research], is rather close to cultural analysis". Therefore, one could reasonably argue that, although NIE does not dismiss broad and abstract phenomena such as organizational culture, OIE allows this kind of phenomena to take a more central role.

\footnotetext{
${ }^{39}$ Scapens (2006) refers to the Enron scandal revealed in 2001 to illustrate the problem of interest with guile.
} 
Hence, Which Institutional Branch Could Provide Better Support for Cost Consciousness?

The main discrepancies between the two Institutional Economic branches have been discussed above. Although this chapter serves as literature review, it provides room for assessing which institutional branch would welcome a theoretical study on the institutionalization of cost consciousness from a management accounting perspective.

As it was highlighted in Chapter 3, cost consciousness seems to be interpreted as a very broad concept; sometimes even connoting a type of organizational culture. Therefore, it seems that OIE might better serve the purposes of this dissertation as NIE seems to allow phenomena (such as group morale, corporate culture, and organizational memories) to take a secondary role in its theories. In studying cost consciousness, the theoretical framework must allow it to serve a more prominent role as the notion is central to this dissertation.

However, a brief review on NIE (aiming to identify at least one central important NIE framework) could provide a better assessment on how this institutional branch could (or could not) support an analysis on the institutionalization of cost consciousness.

\section{A Brief Review on New Institutional Economics}

NIE has spurred great interest during the last decades and its defendants include Nobel Laureates Kenneth Arrow, Ronald Coase, Friedrich Hayek, Gunnar M yrdal, Douglas North, and Herbert Simon. As previously noted, both NIE and OIE are interested in institutions. Moreover, both NIE and OIE also welcome evolutionary theories and combine economics with other social sciences such as law, organizational theory, political science, psychology, sociology and anthropology (Klein, 1998; Williamson, 2000).

According to Klein (1998) NIE's "goal is to explain what institutions are, how they arise, what purposes they serve, how they change, and how - if at all - they should be reformed [...] [and, furthermore,] follows a strict methodological individualism, always couching its explanations in terms of the goals, plans, and actions of individuals".

Much work has been conducted in various areas of NIE interest including the Theory of the Firm, Agency Theory, Transaction Cost Economics (TCE) and Public Policy (Klein, 1998). TCE, for example, moves its attention to the ext post stage of contract by (1) naming and explicating the principal dimensions with respect to which transactions differ; (2) naming and explicating the principal attributes for describing governance structures; (3) effecting a discriminating match, according to which transactions are aligned with governance structures so as to promote adaptation of autonomous and cooperative kinds; and (4) asserting whether the predicted alignments are corroborated by the data (Williamson, 2000).

Williamson's Framework: Institutional Environment and Institutional Arrangements

According to Williamson (1998) the NIE comes in two parts. While part one deals with institutional environment (the rules of the game), part two deals with institutions of governance (the play of the game). The origins of both parts are traced, respectively, to Coase's 1960 (The Problem of Social Cost) and 1937 (The Nature of the Firm) essays. Despite Coase's influence in $\mathrm{NIE}$, the term "New Institutional Economics", however, was actually coined by Williamson in 1975 (Klein 1998). 
Moreover, Davis and North (1971) distinguished between institutional environment and institutional arrangements. The institutional environment refers to the set of rules of political, social, and legal nature that establishes the basis for production, exchange and distribution. Examples of these include rules governing elections, property rights, and the right of contract. More specific examples of these are constitutions and their interpretation by the judiciary, together with the views of a nation's people about the institutions they prefer. This environment can certainly be altered with, for example, the use of referendums to change constitutions. In addition, institutional arrangements refer to the specific guidelines between economic units to mediate particular economic relations. Examples of these institutional arrangements include laws legalizing corporations, business firms, long-term contracts, public bureaucracies and nonprofit organizations (Davis and North, 1971; Klein, 1998). Williamson $(1998,2000)$ uses this distinction as a basis for sketching different levels of social analysis which are presented in Figure 13.

LEVEL

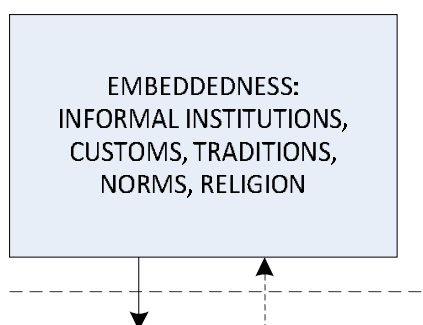

$\nabla$

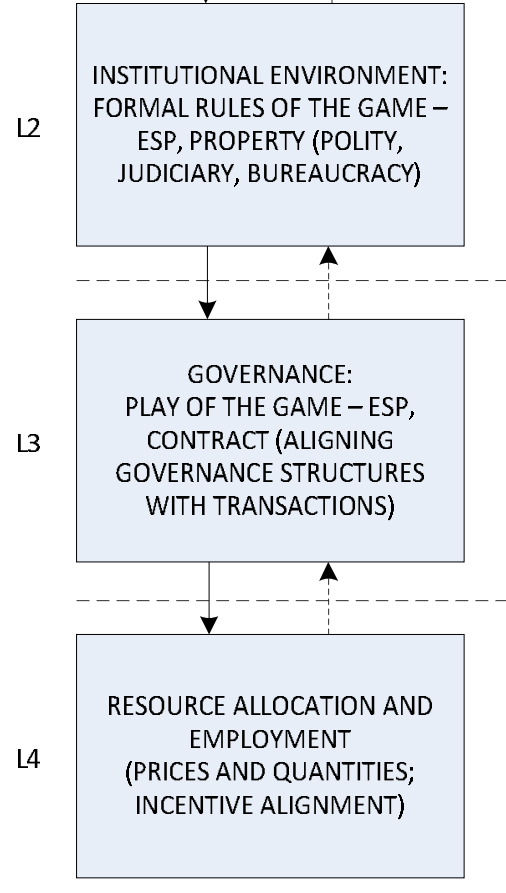

L1: social theory

L2: economics of property rights/positive political theory

L3: transaction cost economics

L4: neoclassical economics/agency theory
FREQUENCY

(YEARS)

PURPOSE

$10^{2}$ TO $10^{3}$

OFTEN NONCALCULATIVE; SPONTANEOUS
GET THE

GOVERNANCE

STRUCTURE RIGHT.

2ND-ORDER

ECONOMIZING
INSTITUTIONAL

ENVIRONMENT RIGHT.

1ST-ORDER

ECONOMIZING

Figure 13. Economics of Institutions (Williamson 1998, 2000)

Consequently, the institutional environment and institutional arrangements previously discussed represent the levels 2 and 3 of this framework which are the main focus of NIE (Klein 1998; Williamson 1998, 2000). I have opted not to be too thorough in the analysis of this framework, 
but I would like to point out some general ideas for clarification purposes, all obtained from the Williamson's 1998 and 2000 papers.

Level 1 is the social embeddedness where the norms, customs, mores, religion and traditions are located. This level has been taken as given by most institutional economists and the institutions at this level change very slowly. Regarding this level, there is room for research focused on identifying and explaining the mechanism through which informal institutions arise and are maintained. Moreover, there is reason to believe that the informal institutions in this top level have mainly spontaneous evolutionary origins which might explain their significant inertia, resulting in their slow level of change. Furthermore, level 2 is the institutional environment that withholds structures which are partly product of evolutionary processes, but also from design opportunities (e.g. constitutions, laws, property rights). "Formal rules" are introduced in this level which provides the opportunity of getting the formal rules right (i.e. first order economizing). Given its partly evolutionary origins, change at this level is also not easy to conduct and history has shown that sharp breaks from established procedures might require civil wars, occupations, military coups or financial crises. As already mentioned, the economics of property rights also act in this level. Furthermore, level 3 withholds the institutions of governance where contract rights are defined and enforced. Although property is important, a perfectly functioning legal system that enforces contracts is not contemplated; therefore the need to come to terms with contract laws rather than an all-purpose law (such as a constitution) is posed. In this level, transaction cost economics, whose origins are commonly traced to the concept of "transaction" by John R. Commons but the theory itself is mainly associated with Oliver E. Williamson (Klein, 1998), has a spotlight position. Finally, level 4 is where neoclassical economics including its marginal analysis work. Moreover, there is the possibility of expanding to a zero level where the study of the mechanisms of the mind can be applied to economics. It is here where evolutionary psychology and cognitive science can contribute. Finally, Klein (1998) and Williamson (1998, 2000) argue that NIE focuses on levels 2 and 3 of the Williamson's framework.

As noted, the dividing line between the Institutional Economic branches is not always so clear. Therefore, as unanimity in identifying the differences with NIE and OIE seems not to exist, pluralism might hold the key for developing institutional theories and frameworks that can provide scholars with better tools for analyzing, for example, the processes of change. Selznick (1996), for instance, calls for reconciliation between old and new institutionalism by noting that the new institutionalism must find its place within the tradition of interest in the vitality and coherence of institutions which was deeply rooted in early institutional economics like John R. Commons. In relation, Williamson (2000, p. 595) states:

I open my discussion of the new institutional economics with a confession, an assertion, and a recommendation. The confession is that we are still very ignorant about institutions. The assertion is that the past quarter century has witnessed enormous progress in the study of institutions. The recommendation is that, awaiting a unified theory, we should be accepting pluralism.

The view that various institutional approaches can complement rather than contradict each other has been recognized by other authors (Karhunen 2008). Consequently, for the purpose of understanding how cost consciousness may be institutionalized in an organization, a pluralist framework would be useful. This dissertation will use the Burns and Scapens Framework (BSF) which draws mainly on OIE, but also on NIE. Moreover, as explained in the introduction of this chapter, the BSF has supported various management accounting studies. Therefore, the 
following section briefly describes certain aspects of NIS and synthesizes the main similarities and differences between the three institutional branches.

\section{New Institutional Sociology and Synthesis}

The discussion about New Institutional Sociology (NIS) aims to provide a very concise review on the historical path of NIS, and, more importantly, highlight how it relates and differs with respect to OIE and NIE. Goodin (1998, p. 4-7) divides the historical path of NIS in four phases. The first phase was characterized by a rather fixation on a standard catalogue of institutions giving as example Herbert Spencer's 1879 Principles of Sociology. Furthermore, the second phase invoked modern sociologists as Pareto, M osca, M ichels, Tönnies, Durkheim, Simmel, Webber and M arx who were all concerned with the ways in which intuitions subsume and subordinate the individual. In addition, the third phase represents the reaction against this collectivism where sociologists proposed to downgrade the importance of collective social structures and institutions in determining the actions and choices of individuals and groups within any given society. Finally, Goodin considers the fourth phase as 'a reaction against that reaction' from new institutional sociologists that come in two groups. The first group includes "radicals who [...] bemoan the ways in which such institutions exercise hidden power over helpless social agents, be they individuals or marginalized groups". The second group consider "individual action [to be] 'embedded' within the context of collective organizations and institutions. Those actions are shaped, and their effects affected and deflected, by the institutional contexts in which they are set".

Furthermore, Goodin (1998, p. 7) explains that although NIS focuses upon ways in which being embedded in collective entities (e.g. the family, the profession, the church, the school, the state) alters individuals' preferences and possibilities; a common objective of social institutionalism, whether its old or new, is to emphasize how individual behaviour is shaped and, perhaps, shapes the larger group setting. In this respect, DiM aggio and Powell (1991, p. 8) state:

The new institutionalism in organization theory and sociology comprises a rejection of rational-actor models, an interest in institutions as independent variables, a turn toward cognitive and cultural explanations, and an interest in properties of supraindividual units of analysis that cannot be reduced to aggregations or direct consequences of individuals' attributes or motives.

Therefore, new institutionalist sociologists distinguish themselves from NIE scholars by rejecting the assumptions of methodological individualism and embracing a more holistic perspective similar to the one welcomed by OIE.

\section{Summary of OIE, NIE, and NIS}

This brief discussion on NIS should serve as a guideline for understanding its most basic tenets, together with its similarities and differences when compared to OIE and NIE. In an attempt to synthesize the ideas, I present a summary gathering the analysis conducted by Scapens (2006) on the differences between these three Institutional branches (Table 10). 
Table 10. Description of three branches of institutional theory according to Scapens (2006)

\begin{tabular}{|c|c|c|c|}
\hline & $\begin{array}{l}\text { New Institutional } \\
\text { Economics }\end{array}$ & New Institutional Sociology & $\begin{array}{l}\text { Old Institutional } \\
\text { Economics }\end{array}$ \\
\hline Description & $\begin{array}{l}\text { Concerned with the } \\
\text { structures used to govern } \\
\text { economic transactions. } \\
\text { It is an extension of the } \\
\text { traditional economic } \\
\text { approach. } \\
\text { Applies assumptions of } \\
\text { economic rationality and } \\
\text { markets to the governance } \\
\text { of organizations }\end{array}$ & $\begin{array}{l}\text { Concerned with the } \\
\text { institutions in the } \\
\text { organizational } \\
\text { environment that shape } \\
\text { organizational structures } \\
\text { and systems } \\
\text { Questions why } \\
\text { organizations look similar, } \\
\text { and what are the pressures } \\
\text { and processes which shape } \\
\text { them }\end{array}$ & $\begin{array}{l}\text { Concerned with the } \\
\text { institutions that shape } \\
\text { the actions and thoughts } \\
\text { of individual agents }\end{array}$ \\
\hline $\begin{array}{l}\text { Related } \\
\text { concepts/ } \\
\text { theories }\end{array}$ & $\begin{array}{l}\text { Transaction Cost } \\
\text { Economics }\end{array}$ & Loose Coupling & - \\
\hline Benefits & $\begin{array}{l}\text { Draws attention to some } \\
\text { of the economic factors } \\
\text { that shape organizational } \\
\text { structures, their control } \\
\text { systems, and management } \\
\text { accounting practices. }\end{array}$ & $\begin{array}{l}\text { Useful in drawing attention } \\
\text { to the need to recognize } \\
\text { the way in which } \\
\text { organizations tend to } \\
\text { conform to what they } \\
\text { perceive as the } \\
\text { expectations of their } \\
\text { broader environment } \\
\text { Similarly to NIE, it can help } \\
\text { us understand the nature } \\
\text { of the external pressures } \\
\text { on organizations }\end{array}$ & $\begin{array}{l}\text { Whereas in NIE and NIS } \\
\text { institutions are given, OIE } \\
\text { enables looking more } \\
\text { closely at institutions } \\
\text { within the organization, } \\
\text { and to focus on the } \\
\text { internal pressures and } \\
\text { constraints that shape } \\
\text { management accounting } \\
\text { practices }\end{array}$ \\
\hline Criticism & $\begin{array}{l}\text { Explaining managerial } \\
\text { behaviour grounded on } \\
\text { bounded rationality and } \\
\text { opportunism, and } \\
\text { particularly on self interest } \\
\text { with guile. } \\
\text { Although it draws } \\
\text { attention to economic } \\
\text { factors, these are only part } \\
\text { of the mish-mash of } \\
\text { interrelated influences (a } \\
\text { look beyond economics is } \\
\text { needed) }\end{array}$ & $\begin{array}{l}\text { Although it draws } \\
\text { attention to external } \\
\text { pressures, not all } \\
\text { organizations will conform } \\
\text { to these pressures and } \\
\text { some may be more } \\
\text { susceptible to certain } \\
\text { pressures rather than to } \\
\text { others (we need to look } \\
\text { within the organizations) }\end{array}$ & - \\
\hline
\end{tabular}

From the previous table one can reasonably infer that for Scapens, both NIS and NIE are problematic in allowing an analysis within organizations. Moreover, Burns (2000) states that, while NIE and NIS assume given institutions, OIE focuses more on the emergence, continuity and change of institutions over time. This is, of course, in contradiction with the statement by Klein (1998) on the goals of NIE. However, the statement by Burns, if taken at face value, is very relevant for this dissertation as this study aims to theorize on how to institutionalize cost consciousness in an organization. Therefore, the institution of cost consciousness is not given.

As the reader may note, the only notion in the figure above which has not been yet discussed refers to loose coupling. Hence, the following subsection provides a brief review on this concept. 
Loose Coupling

$M$ anagement accounting literature has welcomed discussions on the notions of loose coupling (Orton and Weick 1990, Lukka 2007, Nor-Aziah and Scapens, 2007, Cruz et al. 2009; van der Steen 2011) and decoupling (Siti-Nabiha and Scapens 2005). Although widely used, the concept of loosely coupled systems is, however, diversely understood (Orton and Weick 1990, p. 203).

According to van der Steen (2011, p. 532) loose coupling refers to the differences between formal rules aimed at securing legitimacy and actual day-to-day practices. In this sense, routines which deviate from the rules which are supposed to govern them represent an example of loose coupling. Hence, as the BSF can be used to understand the relationship between organizational rules and routines, it can also serve the purpose of recognizing instances of loose coupling.

Loose coupling can occur for various reasons. Cruz et al. (2009, p. 112), for example, state that loose coupling may result from variations introduced by actors confronted by organizational environments compromising multiple logics. Moreover, van der Steen $(2011$, p. 532) claim that loose coupling "can arise from the ongoing reproduction of routines". Furthermore, Lukka (2007, p. 94) discusses the case of a heavy processing equipment business where rules and routines "needed to be, to an adequate extent, loosely coupled from the synchronic perspective". The author argues that the relation between rules and routines at a given point in time can be very loose, and that this loose coupling between rules and routines can be instrumental, at least in the short term, in the smooth functioning of a firm's management accounting. Lukka explains that when trying to change or implement a new management accounting practice, change does not necessarily take place (at least not easily) as attempts to implement a new system tend to encounter resistance as the organizational status quo is disturbed. One option which arises in organizations is to implement a new MAS merely in a ceremonious manner by decoupling it from the day-to-day activities of the organization. In addition, loosely coupled MASs are also studied by Siti-Nabiha and Scapens (2005, p. 48) who explore how the ceremonial implementation of new accounting routines (decoupled from the day to day operation of the business) actually resulted in instrumental change. According to the authors, an organization, or organization key members, attempt to secure legitimacy from external forces by implementing new accounting routines. However, at the same time, organizations (or its key members) decouple these new accounting routines from the day-to-day operations in order to maintain technical efficiency. Nor-Aziah and Scapens state that loose couple systems may serve as a way to protect operations for external interventions.

Summarizing, this subchapter has reviewed some of the differences and similarities between OIE, NIE, and NIS. As noted, the Burns and Scapens Framework (BSF), which is central for the theorization exercise of this dissertation, draws on these three branches. Hence, the following subchapter reviews the BSF together with the concept of management accounting change and some proposals and criticism which have arisen concerning the framework.

\subsection{The Burns and Scapens Framework}

The Burns and Scapens Framework (BSF) is grounded on the duality of action and institutions (very similar to the duality of structure) as it emphasizes that institutions shape the daily interaction of organizational members and, simultaneously, the institutions themselves are the outcomes of such interaction (Burns and Scapens 2000, p. 22). Moreover, the BSF allows the 
analysis of the interactions between actors, sets of rules and routines (i.e. MASs), and institutions. Furthermore, although the BSF draws mainly from OIE, it also includes elements of NIE and NIS. Scapens (2006, pp. 12-13) states that NIE allows drawing attention to the mish mash of economic factors which help to shape organizational structures and management accounting practices. Furthermore, the author argues that NIS allows drawing attention to the need to recognize the way in which organizations tend to conform to what they perceive as the expectations of the environment. Therefore, the BSF seems to provide a proper tool for supporting this dissertation's theorization exercise on the institutionalization of cost consciousness. Hence, this subchapter focuses on reviewing the BSF.

In conducting this review, I will first discuss the foundations of the BSF by reviewing Giddens's model of structuration and Barley and Tolbert's sequential model of institutionalization. Secondly, I will review the BSF by analyzing its elements and structure. Finally, the third section of this subchapter reviews the extensions and proposals for tackling some of the challenges which authors have posited with respect to the BSF.

\section{Giddens and Barley \& Tolbert: The foundations of the Burns and Scapens Framework}

The Burns and Scapens Framework (BSF) has clear foundations on the work by Barley and Tolbert (1997) which is drawn from an NIS perspective (Scapens, 2006). Furthermore, Barley and Tolbert are strongly influenced by Giddens's works $(1979,1984)$ focused on exploring the processes by which structures (i.e. institutions) emerge from action, or influence action. Actually, Barley and Tolbert (1997), in explaining their own mode, present the Giddens's model of structuration and highlight certain deficiencies. Firstly, the authors state that because "Giddens argues that institutions exist only insofar as they are instantiated in everyday activity, critics have charged that he 'conflates' structure with action" (p. 99). The problem of conflation was already noted earlier when discussing the discrepancies with respect to the concept of organizational routines. Secondly, the authors criticize the implicit temporality of Giddens's work despite the fact that he contends that structuration occurs through time (p. 100). Furthermore, Macintosh and Scapens (1990) agree as they note that one of the problems of structuration theory is that it falls short in exploring processes of change. Within this respect, Burns and Scapens (p. 8) explain that without the proper incorporation of time, the examination of the interplay between structure and agency can never be satisfactorily resolved. Thirdly, Barley and Tolbert (1997, p. 112) state that "structuration theory provides little guidance on how to investigate the way in which everyday action revises or reproduces an institution"; hence, researchers "require an empirically viable means of linking the two realms that does not rely on "artistic hermeneutics'" (p. 99). Furthermore, Siti-Nabiha and Scapens (2005, p. 46) argue that that theoretical frameworks should address and analyze questions such as why is there resistance to change? What forces are at work? and How do new systems become embedded in organizations?

Therefore, in an attempt to address these problems, Barley and Tolbert argue that a diachronic model of the structuration process is needed including separate indicators of institutions (which span settings and time) and actions (which are localized to a specific setting) in order to be able to argue that the two (i.e. institutions and actions) map the same principles, or to show how actions implicate structures broader than those of the setting itself. Hence, the authors propose a framework to understand the mechanisms involved in embedding institutions in an organization (Figure 14). This framework does not only elaborate on Giddens work, but also on Berger and Luckmann (1966). 


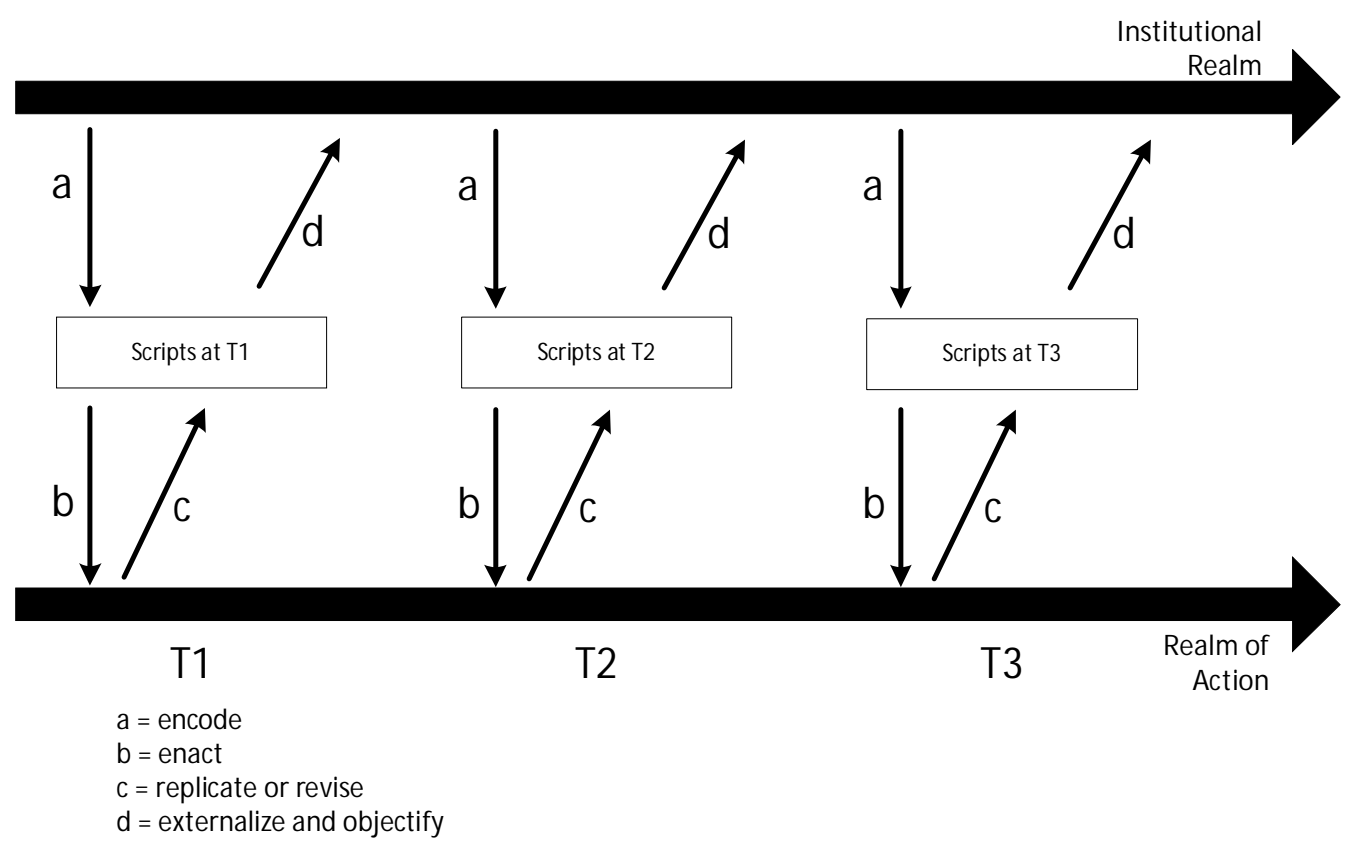

Figure 14. A sequential model of Institutionalization (Barley and Tolbert, 1997)

Scripts ${ }^{40}$ - defined as "observable, recurrent activities and patterns of interaction, characteristic of a particular setting" (Barley and Tolbert, 1997, p. 98) - hold a central role analogous to the way in which modalities act in Giddens's structuration theory. However, Barley and Tolbert (1997, p. 98) argue that scripts "substitute Giddens' more abstract notion of modalities as scripts can be empirically identified, regardless of the type of actor or level of analysis in which a researcher is interested". Levels of analysis in this respect can indicate individuals, groups, organizations or larger collectives.

Barley and Tolbert suggest that institutionalization is a continuous process which can be observed only through time. The model is bounded by the two realms of social structure, institutional and action, the former representing an existing framework of rules and typifications derived from a cumulative history of action and interaction, and the latter referring to actual arrangements of people, objects, and events in the minute-by-minute flow of social life's unfolding (pp. 97-98).

Furthermore, the bold horizontal arrows defining the two realms signify their temporal extensions (Barley and Tolbert, 1997, p. 100). M oreover, the encoding, enacting, replicating and externalizing arrows denote the duality of social systems. While the vertical arrows (encoding and enacting) represent the institutional constraints of action, the diagonal arrows (replication and externalization) represent maintenance or modification of the institution through action.

In addition, it is important to briefly describe the four movements in the framework. First, the encoding of institutional principles in the scripts used in specific settings (i.e. movement a) frequently, but not only, takes place during socialization and involves the internalization of rules and the interpretation of behaviour appropriate for particular settings by individuals. Second, actors also enact (consciously or unconsciously) scripts that encode institutional principles (i.e. movement b). The authors highlight the importance of distinguishing between conscious and unconscious choice for research on how action and institutions relate to each other "since modification of an institution is more likely to require conscious choice than does its

\footnotetext{
${ }^{40}$ Barney and Tolbert (1997) state that scripts encode the social logic of what Goffman (1983) referred to as an 'interaction order' in the American Sociological Association 1982 Presidential Address
} 
reproduction" (Barley and Tolbert, 1997, p. 102). Third, actors also revise and replicate the scripts that inform action (i.e. movement $c$ ). The authors argue that a conscious intention to alter scripts would most likely result in institutional change when compared to unconscious and unintended deviations from the script. The fourth movement refers to the externalization or objectification of the patterned behaviours and interactions produced during a particular period in time. In this way, "patterns acquire a normative, 'factual' quality and their relationship to the existing interests of different actors become obscured" (Barley and Tolbert, 1997, p. 102).

Finally, the Barley and Tolbert framework, however, was not designed specifically for management accounting related studies. Hence, until now, the role which, for example, MASs could play in the framework has not been explained. Very briefly, MASs have been considered to represent sets of organizational rules and routines (Burns and Scapens 2000). Therefore, as rules and routines are central to structuration theory, from where the Barley and Tolbert framework has been drawn, MASs, as sets of rules and routines, may hold a central role in analyzing the processes of organizational change and the dual relationships between the realms of institutions and actions. Moreover, as it has been mentioned, the BSF allows the analysis of the processes of management accounting change. Therefore, the following section reviews the notion of management accounting change before taking a closer look into the BSF.

\section{On Management Accounting Change}

One of the topics which has captured the interest of many management accounting scholars has been change, and, as result, considerable research efforts have been allocated in studying both accounting change and management accounting change since the 1990s (see Ezzamel 1994, Burns and Vaivio 2001, Potter 2005; Busco et al. 2006, Chenhall and Euske 2007, Tuan Mat et al. 2010). Burns (2000) argues that understanding management accounting change can allow managers to be better positioned to influence the path of change programs within their own organizations and anticipate future problems. Hence, understanding management accounting change is important not only for academics but also for non-academic circles.

In an attempt to extend the discussion on the interest for management accounting change, I present the following quote:

[The] study of management accounting change is an interesting locus for experimenting the theoretical advancement of our understanding of accounting as a practice which spans across several realms, from the social to the political via the economic, in forms which are still unclear [...] Theorizing change, accounting and the alike is not a matter which can be confined to accounting and economics as disciplines conventionally conceived. It requires the understanding of ontological and methodological issue which require a tool box much greater and diverse than what accounting researchers seem to have used so far. M anagement accounting change is a laboratory, a theoretical puzzle the solution of which is difficult because there will always be a missing piece which will allow the continuous work around the composition of the picture. Management accounting change is possible because of this incompleteness which makes it an interesting exercise to perform. (Busco et al. 2007, p.146)

Hence, understanding management accounting change can provide multi-disciplinary benefits. Moreover, the previous quote also acknowledges the conceptual difficulty related to this "non- 
uniform" construct (Tuan Mat et al., 2010). In fact, Burns and Vaivio (2001, p. 392) argue that "research on management accounting change can become a traumatic affair".

In providing A Beginner's Guide to management accounting change, Burns and Vaivio (2001, pp. 392-397) offer orientation in understanding the notion of change by presenting three perspectives: (i) the epistemological nature of change which examines if change in fact occurs, if change is progressive or regressive, the dichotomy between change and stability, and the external influences which change may reflect; (ii) the logic of change which evaluates if change has been, for example, managed or unmanaged, linear or nonlinear, systematic or unsystematic, and/or functional or political; and (iii) the management of change which explores the way in which change is managed (centrally or local), how management accounting change is related to organizational culture, how management accounting technologies should be introduced into dynamic and unwelcoming contexts, and if the diffusion of management accounting knowledge has been discrete or dispersed. Therefore, change in itself represents a challenging concept which may be studied from various perspectives - similarly to consciousness, and the approach taken in this dissertation towards the conceptual development of cost consciousness.

Perhaps as a result of the conceptual difficulties which it poses, the discussion on how to study management accounting change has welcomed efforts from various scholars; who might want to deviate from only focusing on what management accounting change is (this approach is clearly aligned with the way in which this dissertation is dealing with cost consciousness). The study of management accounting change has increasingly been conducted during the last years by exploring organizational change as a continuous process (i.e. investigating why and how becomes what it is, or is not, over time), rather than taking the conventional road of analyzing change as discrete outcomes (Scapens and Roberts 1993, Burns and Scapens 2000, Burns 2000). M oreover, according to Scapens (2006), a processual approach grounded on institutional theory allows the study of the "mish-mash" of interrelated influences and, therefore, allows a better understanding of management accounting practices and management accounting change. Therefore, institutional theory allows the understanding of management accounting change.

Furthermore, Shields (1997) notes the existence of two types of drivers of management accounting change: external (environmental factors), and internal (organizationally-concerned) factors. The author, moreover, states that external factors, which cause change in organizations, result on the driving of change in management accounting practices. It is worth noting that Shields does not address the notion of management accounting change per se but, instead, the author discusses change in management accounting practices. Furthermore, the study by Tuan Mat et al. (2010) considers three factors which drive management accounting change: (i) motivational factors such as changes in environment and technology which explain management accounting change and changes in organizational factors; (ii) organizational or contextual factors such as organizational structure and strategy that may be connected to changes in management accounting; and (iii) financial factors which are used as outcomes of management accounting and organizational change. In addition, Tuan $M$ at et al. argue that financial performance may be a determinant or an outcome factor of management accounting change. It is worth noting that the literature review identified financial performance as both a determinant (e.g. financial problems) and a result (e.g. better costing capabilities) related to cost consciousness. Therefore, following this discussion, one could reasonably argue that management accounting change could be considered both a determinant and a result of cost consciousness. 
Moreover, Busco et al. (2007) constructed a model for systematizing the different issues which the literature on management accounting change has been addressing during the last years. The systematization (Figure 15) has four main building blocks (Busco et al. 2007, pp. 126-128).

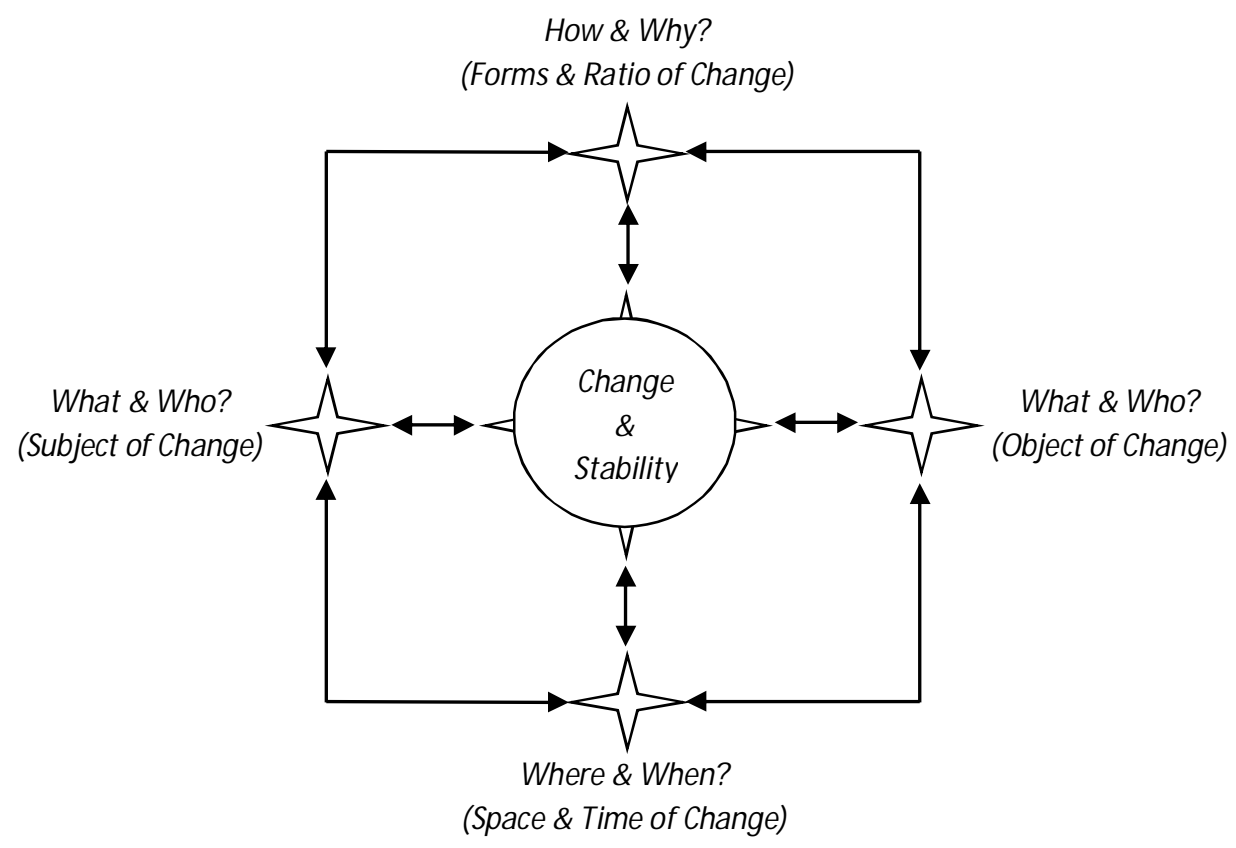

Figure 15. Interpreting management accounting change (Busco et al. 2007, p. 127)

The first building block (i.e. What and Who? on the left hand side of the figure) concerns the forces and/or agents who drive management accounting change. The second building block (i.e. How and Why?) concerns the process through which innovative management accounting ideas are dispersed and successfully embedded in an organization. Typical questions include: How are management accounting techniques able to spread and become practiced? How do they manage to engage practitioners who are driven by different and sometimes opposing agendas? How do they manage to instill hope of solving a problem in the perspective user? and Why this happens? Moreover, the second building block of the systematization sheds light to both change and stability. Change arises as the implementation of a management accounting practice is best described as a translation where innovations are constantly shifted, mediated, and renegotiated. "[M anagement] accounting change always entails a difference, a movement, a variation, in the nature of what is subject to change" (Busco et al. 2007, p. 128). In fact, a stable management accounting practice would be limited with respect to its capability to spread and diffuse in an organization. However, this second building block, paradoxically, also sheds light on the stabilizing nature of management accounting practices. Although translation does entitle change, management accounting practices project, or at least suggest, stability. They do so partly to mobilize the amount of investment which is required to implement a management accounting innovation such as Activity Based Costing or Six Sigma in an organization. In other words, organizations seem to be drawn to the idea of obtaining stability through the implementation of a management accounting practice. The first and second block of this systematization reminds of the framework proposed by Potter (2005) which can be used mainly in three ways including the interrogation of key accounting institutions to enhance our understanding of "how and why the accounting domain may be expanded in some instances" (Potter 2005, p. 280). Furthermore, if the translation mentioned above would be stopped at a moment of time, it would be possible to separate the subjects from the objects of change, which brings forward the ontological difficulty inherent to the concept of management accounting change (i.e. what or who is the subject/object of change) and, simultaneously, introduces the third building block of the 
systematization (What and Who? in the right hand side of Figure 15) concerned particularly with who or what is the object of change. The ontological conundrum, Busco et al. (2007) explain, may be approached in two ways: (i) through an epistemological strategy whereby the difficulty of understanding the object is attributed to the observer (i.e. an object means different things to different people); or (ii) through an ontological strategy where objects per se are considered to be complex. Moreover, the fourth block of the systematization (Where and When?) focuses on where and when management accounting change occurs.

The systematization offered by Busco et al. (2007) reflects the complexity of management accounting change, and, simultaneously, suggests the great potential for research offered by the concept. Moreover, the model also highlights the importance of MASs in all of the building blocks.

Therefore, management accounting change connotes, for example, a change in the way in which management accounting practices are carried on in an organization. This change does not necessarily indicate a change from one management accounting practice to another, as it may also refer to the implementation of a new management accounting system to conduct a particular activity (new or not).

\section{The Burns and Scapens Framework}

This section focuses on reviewing the Burns and Scapens Framework (BSF) which, since its publication on the year 2000, has fueled a considerable amount of academic research. Some of this research uses the framework for supporting management accounting related studies (see Burns 2000, Siti-Nabiha and Scapens 2005, Guerreiro et al. 2006, Hyvönen and Järvinen 2006, Spraakman 2006, Lukka 2007, and ter Bogt 2008). Furthermore, other authors have also proposed extensions for the original framework (see Busco and Scapens 2011, Quinn 2011, and Wanderley et al. 2011). In addition, other authors have also focused on exposing the framework's shortcomings (see Englund and Gerdin 2008, Johansson and Siverbo 2009, and Länsiluoto and Järvenpää 2010).

The developers of the framework explain the general purpose of the BSF:

the framework explores the complex and ongoing relationship between actions and institutions, and demonstrates the importance of organizational routines and institutions in shaping the processes of management accounting change [...] The framework is offered as a starting point for researchers interested in studying management accounting change, and through such studies the framework will be extended and refined (Burns and Scapens 2000, p. 3)

Moreover, the main purposes of the BSF have been reformulated in various ways highlighting the different uses which the framework can serve. From a broad perspective, the BFS studies the institutionalization process (Hyvönen and Järvinen 2006, p. 5) and management accounting change (Spraakman 2006, p. 102). Furthermore, the BSF theorizes management accounting as processes over time by viewing it as a practice infused by rules and routines which has the potential to eventually become institutionalized in an organization (Quinn 2011, p. 339). Also, the BFS explores "the rules-grounded, routinised and potentially taken-for-granted (or "institutionalized") nature of management accounting practices" (Quinn 2011, p. 340). Finally, Spraakman (2006, p. 103) considers that the "disclosed purpose" of the BSF is to "describe and 
explain analytical concepts which can be used for interpretive case studies of management accounting change". Hence, with regards to Spraakman's disclosed purpose, as the study of cost consciousness, as it is formulated in this work, is supported by an interpretative case study, the BSF seems to be a good tool for supporting the analysis on the case study.

The BSF is presented in Figure 16 and, as the reader can note, it resembles very much the model by Barley and Tolbert reviewed on the previous section. The main visible difference between them is the substitution of scripts with the sets of rules and routines.

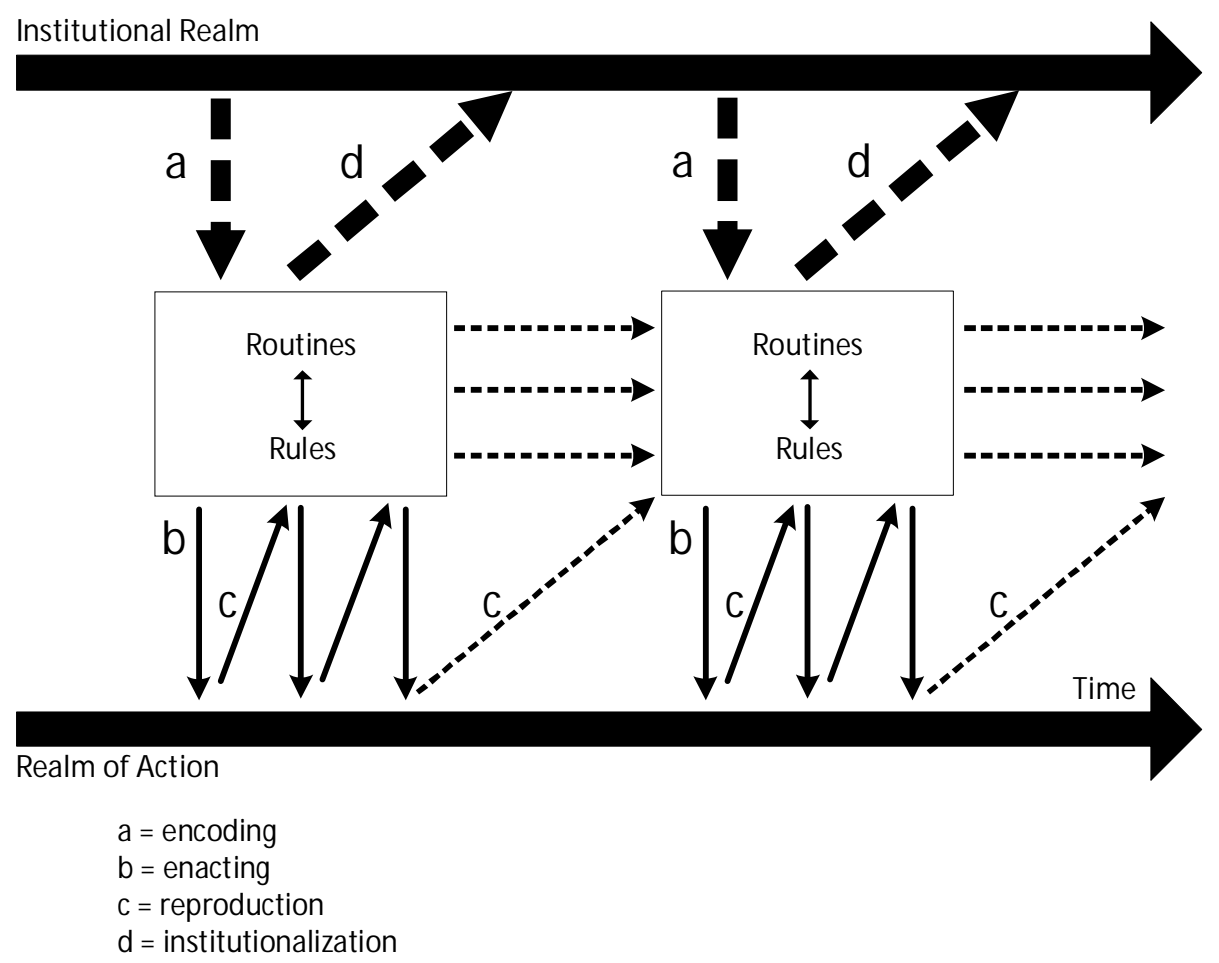

Figure 16. The Burns and Scapens Framework (adapted from Burns and Scapens 2000, p. 9)

The following subsections provide an overview of the different elements and movements which appear in the BSF.

BSF: Realm of Action, Realm of Institution, Rules, and Routines

The BSF is composed of two realms (Institutional and Action) which serve as boundaries of the framework. Moreover, rules and routines interplay not only between each other but also between the Institutional Realm (through the encoding and institutionalization mechanisms), and the Realm of Action (through enacting and reproduction). The complex relationship between the various elements of the framework (e.g. institutions constraining action which, simultaneously, constructs institutions through rules which can become routinised and routines which might be institutionalized) makes the explanation of the framework somehow challenging as it cannot, for example, take a strict reductionist approach. Particularly, the relationship between actors and institutions resembles the complexity of Giddens's duality of structure. In fact, Burns and Scapens (2000, p. 22) state that their framework "is grounded in the duality of action and institutions" as both individuals and institutions are mutually constitutive of each other (Hogdson 1998). 
Table 11. Various definitions of Rules, Routines, and Institutions

\begin{tabular}{|c|c|c|c|}
\hline & Rules & Routines (i.e. Habits of a group) & Institutions \\
\hline 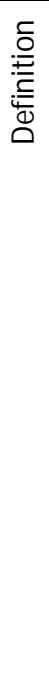 & $\begin{array}{l}\text { The formally recognized way } \\
\text { in which 'things should be } \\
\text { done' (Burns \& Scapens, } \\
\text { 2000). }\end{array}$ & $\begin{array}{l}\text { The way in which things are actually } \\
\text { done (Burns } \& \text { Scapens, 2000); } \\
\text { The procedures actually in use (Burns } \\
\& \text { Scapens, 2000). }\end{array}$ & $\begin{array}{l}\text { The unquestionable way of doing } \\
\text { things (Burns \& Scapens, 2000); } \\
\text { The imposing form and social } \\
\text { coherence upon human activity } \\
\text { through the production and } \\
\text { reproduction of settled habits of } \\
\text { thought and action (Burns \& Scapens, } \\
\text { 2000); } \\
\text { The structural properties which define } \\
\text { the relations between, and the } \\
\text { activities of, the members of particular } \\
\text { social groups or communities (Burns \& } \\
\text { Scapens, 2000); } \\
\text { The taken for granted ways of thinking } \\
\text { in an organization (Scapens, 2006). }\end{array}$ \\
\hline
\end{tabular}

\begin{tabular}{|c|c|c|}
\hline & Rules $\rightarrow$ Routines & Routines $\rightarrow$ Institutions \\
\hline $\begin{array}{l}\frac{c}{0} \\
\stackrel{2}{\bar{n}} \\
\frac{2}{0} \\
\stackrel{0}{0}\end{array}$ & $\begin{array}{l}\text { Routines comprise "programmatic", rules-based } \\
\text { behaviour which is grounded in repeatedly } \\
\text { following such rules. (Burns, 2000). } \\
\text { By repeatedly following rules, behaviour may } \\
\text { become programmatic and based increasingly on } \\
\text { tacit knowledge which the individual acquires } \\
\text { through reflexive monitoring of day-to-day } \\
\text { behavior (Burns and Scapens, 2000). Rules that } \\
\text { become programmatic and based on tacit } \\
\text { knowledge are then described as routines (Burns, } \\
\text { 2000). }\end{array}$ & $\begin{array}{l}\text { Routines, over time, can become increasingly } \\
\text { underpinned by tacit knowledge which individuals acquire } \\
\text { through reflexive monitoring of past behavior, and once } \\
\text { they become widely accepted in the organization, } \\
\text { routines become institutionalized (Burns, 2000); } \\
\text { If, over time, the emerging routines become widely } \\
\text { accepted in the organization such that they become the } \\
\text { unquestionable form of management control, then they } \\
\text { can be said to be institutionalized. (Burns and Scapens, } \\
2000 \text { ); } \\
\text { Institutions are the routines which have become } \\
\text { dissociated from their historical roots-i.e. over time they } \\
\text { have become taken-for-granted (Scapens, 2006). }\end{array}$ \\
\hline
\end{tabular}

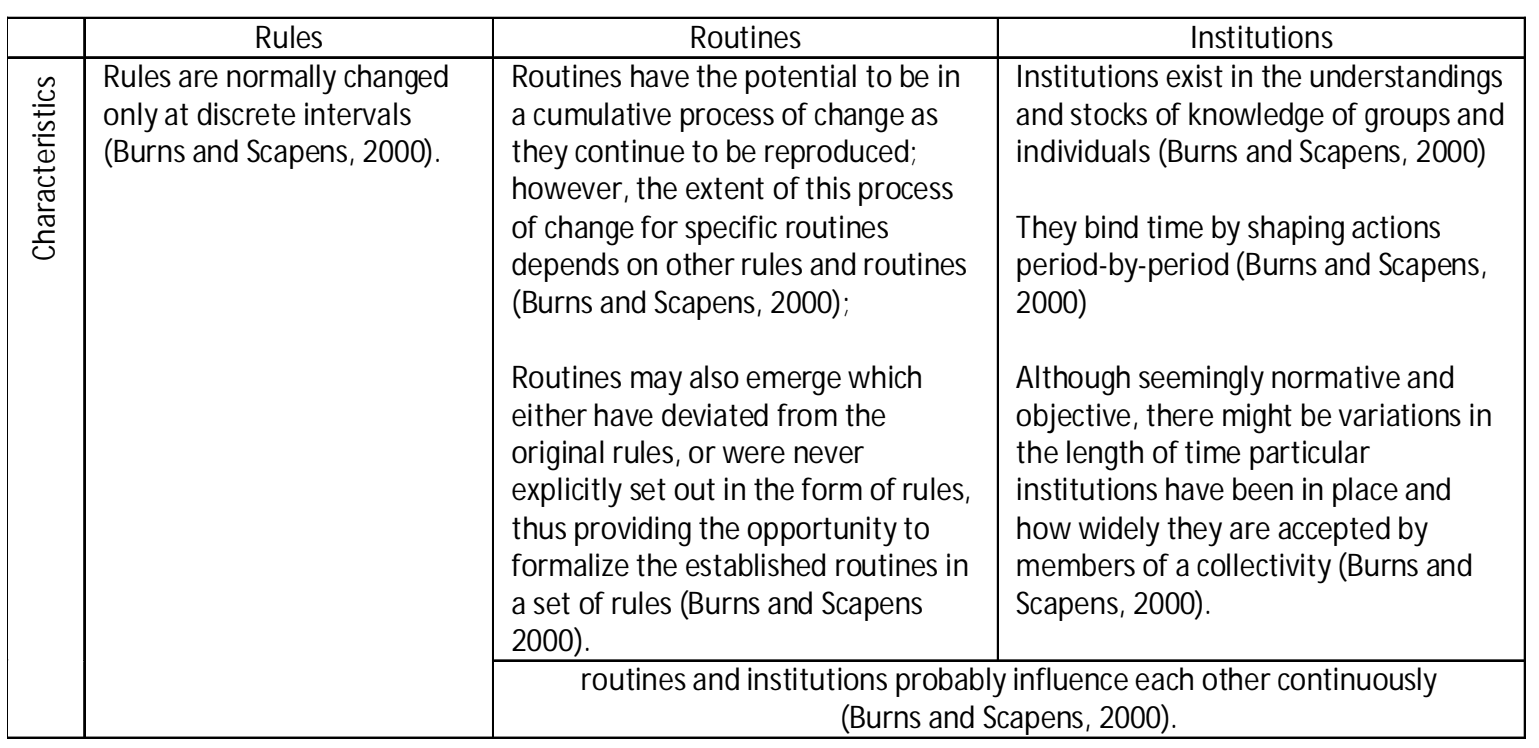

In an attempt to clarify some of the most visible elements of the framework, I have summarized in Table 11 the definitions, characteristics, and relationships between rules, routines and institutions by referencing Burns and Scapens (2000), Burns (2000) and Scapens (2006) to provide consistency as they are the developers of the BSF. 
As the reader may note, these concepts have already been already thoroughly reviewed. Hence, it is not necessary to focus on these at this stage.

Moreover, similarly to the model by Barley and Tolbert, the BSF uses the Institutional Realm at the top-end of the framework and the Realm of Action at the bottom-end. Furthermore, these realms are depicted with arrows suggesting an ongoing and cumulative process of change through time. I believe that this reflects a natural way to set the framework as individuals (placed at the bottom) both draw from and construct the more abstract institutions (at the top). The resemblance to people on earth and the mind boggling immensity of space (or to an 'enlightening' heaven) comes to mind. Moreover, while the Institutional Realm is composed by the institutions of a particular organization, the Realm of Action is composed by the interaction between organizational actors.

Taking a top-down perspective, the dotted lines on the upper part of the figure suggest that institutions are more abstract than the rules and routines. Furthermore, by placing routines above rules, the authors also suggest that routines are more abstract than rules. Nelson and Winter (1982) placed routines one again on the spotlight of organizational literature by highlighting their central role in storing organizational knowledge and, furthermore, in the production and reproduction of organizational memory. Moreover, accounting practices have been described as organizational routines by a considerable amount of scholars (see Roberts and Scapens 1991, Dent 1991, Scapens 1994, Burns and Scapens 2000, Busco et al. 2006, Guerreiro et al. 2006); hence, allowing them to study management accounting practices with the use of both structuration theory and institutional theory.

Moreover, it is important to remember that although rules can be transformed into routines through the process of routinization, this is not always the case, and some routines can be introduced in an organization which do not represent previously routinized rules. In addition, the process of routinization can produce routines that are very different from the original rules. Also, rules are considered by Burns and Scapens as less abstract, therefore closer to the realm of action where individuals interact between each other. Also, the two separate sets of rules and routines in the BSF suggest that every once in a while new rules and new routines may be introduced or, in a more discrete manner, may emerge.

Finally, one could summarize a considerable part of this subsection by stating that individuals (in the Realm of Action), draw from and, simultaneously, constitute the institutions (situated in the Institutional Realm) through sets of rules and routines which symbolize management accounting related ideas, concepts, techniques, and systems (Burns and Scapens 2000, p. 23).

\section{BSF: Encoding, Enacting, Reproduction, and Institutionalization}

The two realms in the BSF are connected by rules and routines by four movements (i.e. encoding, enacting, reproduction, and institutionalization). This is clearly analogous to the way in which Barley and Tolbert's scripts connect actors and institutions. However, while Barley and Tolbert tried to take distance from Giddens's modalities, Burns and Scapens (2000, p. 10) argued that their framework "illustrates the way in which rules and routines act as the modalities which link the institutional realm and the realm of action". 
The four movements provide a combination of two types of elements: synchronic (institutions containing and shaping action at a specific point in time), and diachronic (actions producing and reproducing institutions through their cumulative influence over time). It should be noted that the choice of the terms synchronic and diachronic highlights once again the recurrent referral to language by institutional and structural scholars. The Oxford Dictionary of English (2006) defines the synchronic as "concerned with something, especially a language, as it exists at one point in time", and the former as "concerned with the way in which something, especially language, has developed and evolved through time".

Furthermore, although the movements are practically identical as the ones in Barley and Tolbert's model, I would like to briefly rescue Burns and Scapen's explanation on the matter as the BSF is the framework which is central to this dissertation. First, encoding (i.e. arrow a) refers to the encoding of institutional principles into rules and routines, where, generally, existing routines will encode the prevailing institutional principles and will shape new rules that will eventually lead to the (re)formation of the existing routines. The encoding process draws on the institutional principles of taken for granted assumptions through the instantiation of existing meaning, values and power. Hence, Giddens's three dimensions of structure (i.e. signification, legitimation, and domination) are represented in the encoding process.

Second, enacting (i.e. arrow b) involves the actors enacting the rules and routines which encode the institutional principles. This movement may involve conscious choice, but will usually result from reflexive monitoring and the application of tacit knowledge about how things are done. The enactment of rules and routines may be object to resistance if these challenge existing meanings and values and actors have sufficient power to intervene. However, change, particularly with respect to routines, is unlikely in the absence of external changes (i.e. advances in technology). Furthermore, resistance is an issue which Burns and Scapens (2000, p. 17) focus on as they note three main types of resistance (i) formal and overt resistance due to competing interests; (ii) resistance due to the actors not having the capacity or knowledge to implement the change; and (iii) resistance arising from mental allegiance to specific ways of thinking and doing in the organization.

Third, reproduction takes place as repeated behaviour leads to a reproduction of routines which may involve either conscious or unconscious/unintended change. During reproduction (i.e. c), if actors have (or obtain) sufficient power, then conscious change might occur. Similarly, unconscious change may occur if the rules and routines are not sufficiently understood and/or accepted by actors where there is absence of systems which monitor the execution of the rules and routines. Furthermore, I would like to take note of the three types of change which Burns and Scapens (2000, pp. 18-21) highlight (i.e. formal versus informal change, revolutionary versus evolutionary change, and regressive versus progressive change) and which have already been previously reviewed. In relation to this, when discussing the notion of loose coupling, it was mentioned that organizational actors may resist the implementation of a new MAS by implementing it merely in a ceremonial manner. According to the Burns and Scapens (p. 20), ceremonial implementations are intricately related to regressive change as they restrict institutional change.

Fourth, institutionalization refers to the institutionalization of rules and routines which have been reproduced through the behaviour of the individual actors. In this way, the rules and routines become institutions (i.e. simply the way things are). Moreover, these institutions will then be encoded into the ongoing rules and routines and will shape new rules. As institutionalization occurs, a disassociation of rules and routines from their historical 
circumstances takes place, highlighting once again the ideas of path-dependence and lock-inhistory which are intricately related to evolutionary types of processes.

It is also worth mentioning that the greater amount of arrows on the bottom part of the figure (between the Realm of Action and the sets of rules and routines) when compared to the arrows at the upper part (between the Institutional Realm and the Rules and Routines) suggest that changes in the Realm of Action occur more frequently when compared to changes in the institutional realm. In addition, the dotted horizontal lines between the two boxes suggest that, even if the BSF provides a simplistic view of the relationship between its elements, one should not view the relationship between rules and routines in a strictly deterministic way as the implementation of new rules and the emergence of new routines is influenced by both the encoding of institutions and the reproduction of existing routines (Burns and Scapens, 2000, p. 11).

Before finishing this review on the BSF, it is worth noting that no 'user-manual' was proposed by its developers. However, Soin et al. (2005, p. 255) suggest, as first step, the identification of the institutional realm by identifying the initial set of routines and rules that characterize management accounting control in an organization before, for example, the implementation of a particular system ${ }^{41}$. The idea is that, as the rules and routines of an organization have been encoded by the prevailing institutions, then, by analyzing the rules and routines, one may decipher certain institutions of an organization. Soin et al. however, abstain from providing subsequent steps in using the BSF. However, one may infer that the second step is the identification of the main actors and their relationship with the wider institutional realm. Third, it is important to identify the movements by, for example, describing who is involved in the encoding, enactment, reproduction, and institutionalization processes related to the processes of management accounting change.

The review on the BSF, as it was originally published, has drawn to an end. However, proposals for extensions and modifications to the framework have arisen recently.

\section{Use of the BSF in previous studies}

In an attempt to clarify how the BSF has been used in the past, I present a modified version of the BSF (Figure 17). This modified version clarifies that M anagement Accounting Systems (MASs) are considered as sets of rules and routines. This, moreover, highlights the role of MASs in organizational change, a relationship which has already been discussed in scientific literature (Campanale et al. 2010).

${ }^{41}$ Guerreiro et al. (2006, p. 218) also follow this first step. 


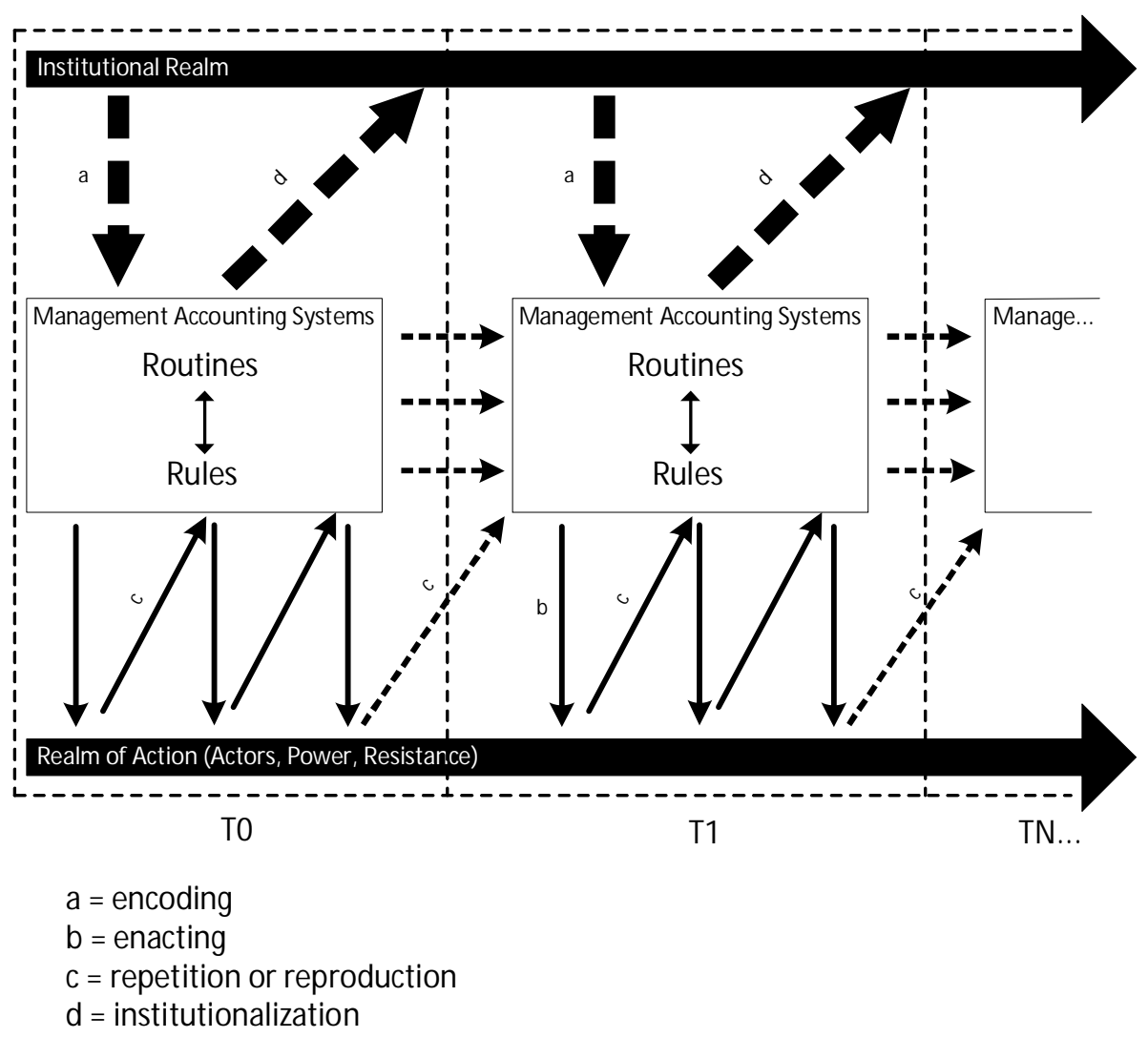

Figure 17. Modified BSF for illustrating its use

I will illustrate how this proposal serves the use of the BSF by very briefly exploring some studies which have used the BSF to analyze the process of management accounting change.

As the studies reviewed in this section analyze the changes which arise from the implementation and/or modification of MASs; the extension depicted above differentiates time before and after the implementation or modification of the MAS. Although time appeared explicitly in the Barley and Tolbert model, it was discarded in the BSF and reinstituted by Busco and Scapens (2011). Therefore, the elements and processes before the implementation/modification of the MAS reside in TO. Moreover, the elements and processes after the implementation/modification reside in $\mathrm{Tl}$.

Furthermore, I will organize the results of each review into four groups. The first group refers to the Institutional Realm and includes the institutions which authors identify and/or analyze. Moreover, drawing on Giddens, I will refer to the second group as modalities. As MAS are considered to be sets of rules and routines, I will not only identify the MAS, but also the particular rules and routines which the authors of the studies describe. In addition, the third group refers to the Realm of Action listing the main actors involved in the processes of management accounting change; the movements (i.e. encoding, enactment, repetition, and institutionalization) described on each case; the types of power ${ }^{42}$ which the actors inflict in the organization (i.e. power over resources, power over meaning, and power of the system); and/or the sources of resistance ${ }^{43}$ which could be present (i.e. formal and overt resistance due to competing interests; resistance due to the actors not having the capacity or knowledge to implement the change; and resistance arising from mental allegiance to specific ways of thinking

\footnotetext{
${ }^{42}$ According to Hardy (1996)

${ }^{43}$ M entioned by Burns and Scapens (2000, p. 17)
} 
and doing in the organization). Finally, the fourth group refers to the three dichotomies of change ${ }^{44}$ (i.e. regressive vs. progressive, revolutionary vs. evolutionary, and formal vs. informal).

In an attempt to avoid mistakes resulting from my personal interpretation, I will identify only those elements which the authors clearly describe. This means that I will not take liberties in referring, for example, to a particular practice (e.g. budgeting) as a routine unless the authors clearly identify it as such.

Burns and Scapens (2000): Two cases which illustrate the use of the BSF

Burns and Scapens (2000) refer to two case studies in illustrating the essential features of the BSF. The first case study explores a company called Ferac Plastics where financially literate managers held high regard to accounting information. The review of this case taking the steps described above can be summarized in the following way:

- Institutions (T0): Accounting routines (through a lengthy institutionalization process) which became disassociated from their historical origin therefore becoming taken-forgranted. However, M anagement accounting was not institutionalized in one division.

- Modalities:

- Modality (T1):

* Routines (T1): Accounting routines evolved during the processes of enactment and reproduction.

- Realm of Action (T0 $\rightarrow T 1$ ):

- Actors:

* Managers

- Movements:

* Encoding (T0): accounting routines which were part of the managers' stock of knowledge encoded accounting based assumptions.

* Enacting (T0): M anagers enacted these routines on a daily basis.

* Enactment \& Repetition (T0): Accounting routines evolved as managers found acceptable ways of working.

- Change (T1):

- Formal vs. Informal. Informal change in the organizational and accounting routines occurred in order to deal with detrimental effects on production and tensions which arose between managers.

In discussing this case, the authors argued that accounting routines are not inevitably institutionalized. However, the routines in this case study are not clearly identified; therefore suggesting that the routines and the institutions refer to the same accounting routines.

The second case study explored a division of a company named Omega plc where a project named the Production Cost Control Project (PCCP) was implemented to improve the flow of accounting information shared between operating units and divisional managers. However, divisional unit managers, as opposed to accountants, were not financial literate and preferred to use more production-based meanings and routines; a situation which resulted in conflict between these actors. Using a similar approach as on the previous case review, the main elements of the BSF can be summarized in the following way:

\footnotetext{
${ }^{44}$ Highlighted by Burns and Scapens (2000, p. 18)
} 
- Institutional Realm (TO): Different meanings and norms embedded in the emerging organizational routines.

- Modalities:

- Modality (T1):

- Management accounting system: PCCP.

- Realm of Action:

- Actors:

* Divisional Managers

* Accountants.

- Movements:

* Encoding (TO): Managers' routines encoded the different institutions which resulted in different understandings of PCCP

* Encoding (T1): The encoding of accountants' assumptions challenged the routines which were encoded by production-based institutions. The challenge was resisted and resulted in the failure of the project.

- Resistance:

* Resistance due to mental allegiance. Managers were reluctant to

- Change (T1): conform to the new modes of thinking and behaviour.

- Formal vs. informal. Problems with formal change may arise whenever a change in ways of thinking (which is the essence of informal change) is not accompanying the overall change process.

In comparing both cases, Burns and Scapens (2000, p. 16) concluded that it might be "easier to introduce changes which do not challenge existing routines and institutions". Moreover, it is possible to see from the summaries above, that the resistance exerted by the actors was an important focus of the second case when compared to the first. Furthermore, the first case allowed the identification of the repetition movement which was not evident in the second study. Therefore, these acknowledgements serve as further evidence of the way in which this particular organization of the results of the BSF allows the identification of points of interest in case studies which use the framework.

\section{Burns (2000): the institutionalization of Results Orientation}

Burns (2000) explores accounting change in a chemical company called Becks where he describes the efforts of the Managing Director (MD) to institutionalize a results orientation mindset (which had been successfully embedded in most of the organization a few years before) in the Product Development Department (PDD) of the company by implementing certain accounting rules and routines.

- Institutional Realm:

- Institutions (and routines) (T0): Shared assumptions with respect to importance of yields, efficiencies and contributions. Accounting was embedded in the management process resulting in many accounting related activities being conducted by non-accountant managers. Results-orientation is underpinned by the widespread use of accounting practices and their routinised nature (e.g. compilation and use of budgets, forecasts and other contribution schedules). There is an implicit relationship between results orientation and a need to make money and contribute towards results. 
- Institutions/Institutionalization (T1): The new accounting routines failed to institutionalize results orientation.

- Modalities:

- Modality (T1):

* Management Accounting System: New accounting procedures (e.g. the completion and regular analysis of time sheet data demanded on a weekly basis, a development report) were introduced with the aim of increasing the awareness of the impact of the PDD behaviour on the overall profitability and on product prioritization.

* Routinization: Collection of timesheet data, running off the new accounting schedules, monthly review meetings) occurred.

- Realm of Action:

- Actors:

* Managing Director (MD)

* Chief Chemist (CC)

* Other members of the PPD

- Movements:

* Encoding (TO): Accounting routines are encoded into the organisational system

- Power:

* Power over resources (T0 $\rightarrow \mathrm{T} 1)$ : the MD drew on his authority to force behaviour modification in PDD

* Power over decision making ( $\mathrm{T} 0 \rightarrow \mathrm{T} 1$ ): the MD dominated the process of determining what ought to be done with PDD before and after the implementation

* Power over meanings (T0 $\rightarrow \mathrm{T} 1$ ): exercised at Board level, by for example, the MD challenging colleagues to stimulate senior personnel to look things in a particular way.

This case is particularly helpful for explaining why the institutionalization of cost consciousness could potentially be studied with the use of the Burns and Scapens framework because cost consciousness and 'result orientation' hold two relevant similarities. First, both are abstract notions. Burns (2000), actually, does not allocate much effort in explaining what 'result orientation' means. However, he provides hints throughout the paper by mentioning that result orientation has something to do with 'making money' and 'contributing towards results'. Second, results orientation was institutionalized by routinizing and institutionalizing management accounting practices. Therefore, one could argue that management accounting practices were drivers, or determinants, of result orientation. This is analogous to the results identified by the literature review on cost consciousness.

Furthermore, the organizational structure used to review the work by Burns allows one to easily identify power as an important focus of the study. Moreover, it is also possible to observe that, when compared to the cases presented by Burns and Scapens, the institutions and the process of routinization were also considered in greater detail.

Soin et al. (2002): ABC as the set of rules and routines for establishing change

Soin et al. (2002) describe the case of the implementation of an Activity-Based-Costing (ABC) system in a department of a UK multinational bank as a response to the need "to become more 
explicitly profit and cost conscious" and the surge of "new human resource management practices" (Soin et al. 2002, p. 257).

- Institutional Realm:

- Institutions (T0): The use of multiple control technologies where personnel was more important than management accounting

- Institutions (T1): ABC introduces accounting into management culture.

- Modalities:

- Modality (TO):

* Routines: A great deal of the work process in the Clearing department was routines and countable.

- Modality (T1):

* Management Accounting System: ABC system

- Realm of Action:

- Actors:

* Internal team of the bank staff: Implementation

* A team of management consultants

- Movements:

* The Human Resource Engineering (HRE) group

* Encoding (T0 $\rightarrow \mathrm{T} 1)$ : Three teams involved in encoding new principles

* Enactment (T1): Three teams involved in enactment

* Repetition and Institutionalization (T1): ABC and HRE teams

- Change. There was evidence of change in the three types of dichotomies:

- Formal vs. Informal. The implementation of $A B C$ is a formal change. Moreover, non accountants appreciating financial information, and $A B C$ accountants appreciating operational issues are examples of informal change

- Revolutionary vs. Evolutionary. The exposure to management accounting information in the banking world was limited, therefore the change can be considered as revolutionary

- Progressive vs. Regressive. The reluctance and incompetency to employ the full potential of $A B C$ is an example of regressive change.

The case explored by Soin et al. is interesting for this dissertation for a couple of reasons. First, it supports the idea that organizational culture as a set of institutions providing further support to the discussion on the relationship of organizational culture and institutions previously held. Second, cost consciousness is mentioned in this case study, although it seems unclear if it can be considered as the driver of change or as part of the resulting institutions.

Moreover, the organization of the discussion and results by Soin et al. show that the authors took special interest in the types of change which occurred during the process of management accounting change. Furthermore, the authors also provide a clear relationship between the four movements and the specific actors involved with them.

Siti-Nabiha and Scapens (2005) the institutionalization of Product Orientation

Siti-Nabiha and Scapens (2005) provide an additional example of how the institutionalization of an abstract organizational phenomena or culture can be studied through the exploration of institutions expressed in terms of product orientation. The setting takes place in Eagle, a gas processing company in an East Asian country whose parent company is called the National Oil 
Company (NOC). In this case, the company implemented a new system called Value Based Management (VBM) which was underpinned by financial orientation.

- Institutional Realm:

- Institutional Realm (T0): Product orientation providing basis for managers' decision making and shaping the organization's goals. Production orientation has been reinforced by the prioritization of plant reliability over, for example, maintaining a market share or efficient operations. Moreover, this institution resulted in accounting being seen as an instrument for securing means for production, rather than as a tool for financial control.

- Institutional Realm (T1): Production orientation prevailed even if the VBM implementation was expected to institutionalize financial-orientation.

- Modalities:

- Modality (TO):

* Routines: Budgets and budget reviews

- Modality (T1):

* Management Accounting System:

- The Value-Based Management (VBM) which had financially oriented ways of thinking 'its heart' (p. 52). Moreover, the VBM required managers to formulate key performance indicators (KPIs). However, this system was implemented only ceremonially.

- The Performance Measurement System (PMS). This system used KPIs for performance evaluation and results and resulted from an early formulation of two different sets of KPIs during the implementation of VBM. The implementation of this system resulted from the ceremonial implementation of VBM .

* Routines: KPIs (e.g. monthly collection of data for them, producing management reports containing them).

- Decoupling: Rules were implemented and routinized in a ceremonial manner from the day-to-day operational activities.

- Realm of Action:

- Actors:

* Eagle's managers and accountants

* NOC's chairmen

* Consultants (who recommended VBM)

- Resistance:

- Resistance due to lack of capability. Prior to the implementation of KPIs, the managers had not experience of constructing indicators of their performance.

* Resistance due to mental allegiance. There was a feeling that financially oriented indicators could not fully reflect the complexity of the roles and activities of managers.

- Change:

- Regressive vs. progressive: There is evidence of both as resistance to the implementation of VBM indicates regressive change. Actually, the authors claim that the VBM was implemented only ceremonially. The formulation of alternative KPIs, however, shows that progressive change also occurred. 
- Formal vs. informal: informal change occurred once the PMS (based on informal KPIs) was in place. Formal change also occurred with the introduction of the VBM KPIs.

- Revolutionary vs. evolutionary: While the introduction of VBM could be regarded as revolutionary with respect to its impact on the existing institutions, the authors seem to suggest that it is really an example of evolutionary change (pp. $65-66)$.

In the discussion of the institutions governing Eagle, the authors note that although research suggests that major external events can lead to the questioning of previously held assumptions, the Asian economic crisis did not impact the product orientation mentality of the company even if there was an "awareness of the need to be more cost conscious" (Siti-Nabiha and Scapens 2005, p. 53). Moreover, in the case of Eagle, one reason for the failure to drive cost consciousness could have been that "gas became a cheaper alternative relative to other sources of power" (Siti-Nabiha and Scapens 2005, p. 53). Furthermore, the case above highlights how the implementation of a modality which is not aligned with prevailing institutions can result in its ceremonial implementation and, furthermore, the implementation of another modified modality which does not disturb the taken-for-granted assumptions of the organization.

The organization of the results of the case study by Siti-Nabiha and Scapens allows one to easily notice that both resistance and change were particular areas of interest. Moreover, it also shows that an instance of decoupling was identified and discussed in the study. Furthermore, the summary also highlights the fact that two MASs arose after the intended management accounting change process took place.

Guerreiro et al. (2006): the institutionalization of M anagement Accounting

This case study explores management accounting change in a large Brazilian bank. The article by Guerreiro et al. (2006) takes an organizational approach similar to the one presented in this chapter by clearly identifying some concepts and artifacts before and after the management accounting change process took place (see Guerreiro et al. 2006, p. 213). Therefore, the description below will not concentrate on these but on the other elements of the BSF which were not identified by the authors.

- Institutional Realm:

- Institutional Realm (T1):

- New principles included optimization of economic performance of the organization, improvement in managers' operational and economic performance, improvement in managers' accountability, and improvement in organisational control.

- However, the authors argue that management accounting or the management accounting change process (p. 224) per se represents the new institution

- Modalities (see Table III in Guerreiro et al. 2006, p. 213):

- Modality (TO):

* Management Accounting System: A system inadequate for accountability purposes which was under-used and disregarded by managers

- Modality (T1): 
* Management Accounting System: a new management accounting model based on economic measuring concepts.

* Artifacts: simulation system, budget system, result determination system, procedural manuals, meetings of planning process, performance evaluation system, committee of investments, and the committee of new products.

- Realm of Action:

- Actors:

* Controllership unit which promoted integration and the evaluation and control of the overall economic performance.

* Board of Directors and Executive Board of the bank

* Project team

- Movements:

* Encoding: The project team designed the new routines driven by the new institutional principles

* Enacting: The enactment of the new model seemed to be carried out by managers in the business areas (with weak resistance) and in the central service areas (with strong resistance). Moreover, enactment was powered by efforts of training and diffusion.

* Reproduction: Managers made periodical presentations of management to the Board of Directors and to the Executive Board

* Institutionalization: The controllership area unit introduced and consolidated various concepts which became institutionalized. The management accounting change process became institutionalized. While management accounting concepts were highly institutionalized inside the members of the bank's board and managers of business areas, the level of institutionalization was low-moderate inside the group of managers of central service areas.

- Resistance:

* Resistance due to mental allegiance. M anagers' reluctance in conforming to the new form of performance measurement and due to the difficulties

- Changes: in operating with new concepts.

- Revolutionary vs. Evolutionary: As the new concepts in the new management accounting system where considerably different from the old, revolutionary change occurred.

The article by Guerreiro et al. (2006) provides a comprehensive use of the BSF with respect to the identification of the various elements which compose it. The authors described thoroughly the two realms and modalities. However, the authors seemed to have abstained themselves from developing a deeper discussion with respect to change as they only seemed to briefly analyze one dichotomy (i.e. revolutionary vs. evolutionary). Clearly, formal change occurred with the implementation of the new accounting model. Moreover, it is reasonable to believe that the resistance to change described in this study could have probably resulted in some sort of regressive change. Furthermore, the way in which the results of the study by Guerreiro et al. have been presented above, clearly highlights that, apart from focusing on the modalities, the authors emphasized the importance of the role of the actors in the four movements. 
In sum, the five reviews previously discussed have brought five main issues to light. First, these discussions have shown the versatility of the BSF. While Burns and Scapens (2000) used its cases for illustrating how the BSF could be used, Burns (2000) placed particular focus on the analysis of the different forms of power. Moreover, Soin et al. (2002) and Siti-Nabiha and Scapens (2005) used the BSF for analyzing the dichotomies of change. Finally, Guerreiro et al. (2006) provided thorough descriptions of the modalities and movements involved in their study.

Second, these discussions provide evidence of the use of the BSF to study the institutionalization of abstract phenomena which have linguistic similarities to cost consciousness (e.g. results orientation, production orientation and financial orientation). In addition, and perhaps more importantly, these notions might represent similar types of organizational phenomena. Moreover, neither Burns nor Siti-Nabiha and Scapens develop a thorough explanation of what these concepts connote. Nevertheless, analogously with respect to the case of cost consciousness, readers probably have some sort of idea about what the concepts could mean.

Third, the synthesis and organization of results in the way which has been conducted in this section allows the identification and analysis of elements and processes which:

- Are evident from the BSF (i.e. Institutional Realm, rules, routines, Realm of Action, encoding, enacting, reproduction, and institutionalization);

- Are not evident in the BSF but that Burns and Scapens highlighted when first publishing the framework (e.g. the processes of routinization and institutionalization; the types of resistance; the dichotomies of change; and management accounting systems);

- Were neither explicit in the BSF nor mentioned by Burns and Scapens in their work (e.g. list of institutions, list of actors, types of power).

Fourth, by allowing a clear distinction of the elements of the framework before and after a particular event which clearly demarks management accounting change, the identification of changes in those elements can be easier to notice. Fifth, this extension is better suited for longitudinal studies where the researcher has been able to obtain enough information to describe the framework elements (e.g. routines, institutions) before management accounting change takes place (e.g. ABC is implemented).

The reviews held in this section, therefore, have collected and highlighted the elements and processes which have interacted in the processes of management accounting change studied with the BSF. However, these studies, as were conducted from the year 2000 to the year 2006 did not take into the account the proposals which were published in later years. Hence, the following section reviews the additional elements and processes which could play a role in the institutionalization of cost consciousness.

\section{Extensions and Modifications of the BSF}

This section reviews the works by Länsiluoto and Järvenpää (2010), Busco and Scapens (2011), and Quinn (2011), as they provide interesting modifications to the BSF which serve the purposes of this dissertation. 
Quinn incorporates the Ostensive \& Performative Aspects of Routines

As the reader may remember, the framework by van der Steen (2011) - drawing from the work by Feldman and Pentland (2003) -highlights the recurrent relation between two aspects of routines: ostensive and performative. The framework depicts routines as reproductions of ongoing systems of interaction where the ostensive aspect (e.g. an artifact specifying the routines) guides, accounts and/or refers the performative aspect (i.e. the actual enactment of the routine) that, consequently, creates, maintains and/or modifies the ostensive aspect.

Quinn (2011, p. 338) argues that the conceptual problem with respect to (management accounting) routines arises from the Burns and Scapens (2000) article. Quinn argues that Burns and Scapen's simplistic definitions of routines (i.e. the way things are actually done), together with their design of the framework (e.g. particularly with respect to the placement of routines as close to the abstract institutional realm), result in a conceptual dilemma: on the one hand routines are considered to be considerably abstract, but on the other hand they represent the way in which things are actually done.

Quinn draws on the recurrent relation between the two aspects of routines to propose an extension to the Burns and Scapens (Figure 18).

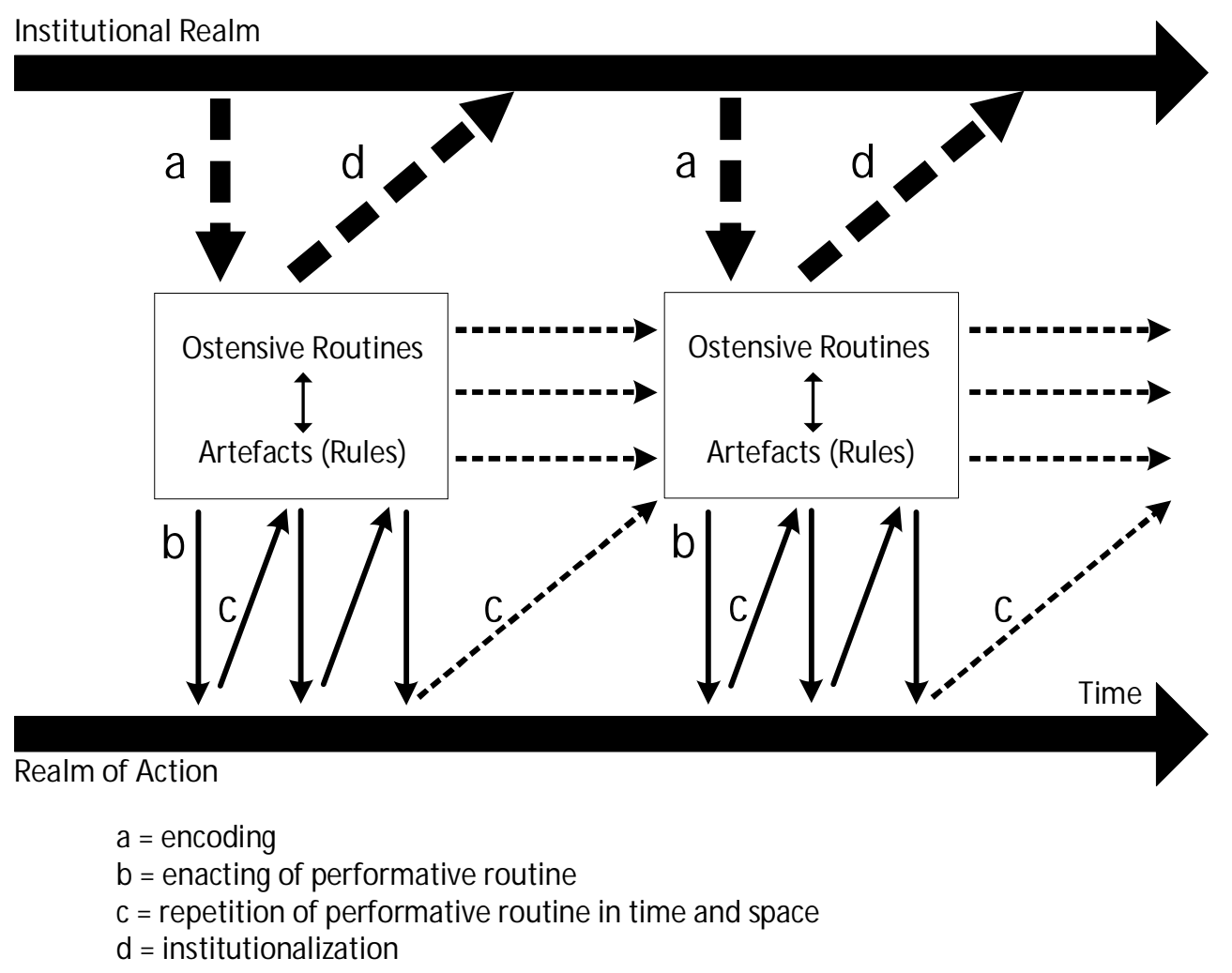

Figure 18. Burns and Scapens Framework extension (adapted from Quinn 2011, p. 345)

Quinn (2011, p. 349) proposes rules to be defined as "a physical representation of a routine, which are formalised in a documented fashion and serve to guide action". In the case in which rules adhering to this definition do not exist, the ostensive dimension of a routine serves as a kind of substitute for rules. Within this logic, rules may be regarded as the visible representation of the ostensive aspect of routines.

In addition, Quinn (2011, p. 347) states that the ostensive aspect of a routine may be represented by various sets of artifacts. The case study analyzed in this dissertation involves a MAS which does not have a formal set of rules guiding its use (i.e. a user's manual). However, the 
MAS which the company has is an Excel spreadsheet, which, according to Quinn (2011, p. 349) can be considered as a rule per se "since actors can refer to it for guidance".

Furthermore, by integrating the works by van der Steen (2011) and Pentland and Feldman (2003) inside the BSF, Quinn provides a solution for conducting case study research in organizations lacking a formalized set of rules; a situation very common in the case of MASs in Small and Medium Enterprises (SM Es) (Quinn 2011, p. 351).

Hence, Quinn's proposed extension may allow a more comprehensive use of the BSF by specifying what researchers should focus when trying to analyze the enacting and repetition movements.

\section{Länsiluoto and Järvenpää: A Closer Look at Power, Status, and Influence in Agency}

Discussions which have enriched the BSF have not only focused on the role and interaction between rules, routines and modalities. According to Länsiluoto and Järvenpää (2010, pp. 201202 ), the BSF lacks consideration for the role of actors during the process of change. The authors argue that, although the role of actors is important, the BSF suggests that they affect in some way the implementation of management systems. In particular, the authors state that the BSF lacks a more comprehensive view on the way power, status, and influence affect the processes of change.

Therefore, Länsiluoto and Järvenpää (2010) draw on the work by Gibson and Earley (2007) and Lovaglia et al. (2003) to discuss the roles of different actors in terms of power, status, and influence. Lovaglia et al. (2003, p. 109) define power as "the structurally determined potential for obtaining favored payoffs in relations where interests are opposed"; influence as what occurs "when a person's opinion or behavior changes to conform to the suggestion of another without the threat of punishment or the promise of reward"; and status as "a person's position in a group's prestige hierarchy". Defining influence is important, the authors argue, as an individual's status determines the actor's influence. Moreover, Lovaglia et al. (2003) develop a conceptual model of collective action theory to develop eleven propositions and four derivations which construct the theoretical relationship between power and status to derive testable hypotheses (Appendix D).

In analyzing actors, Länsiluoto and Järvenpää (2010) present three tables which summarize important aspects of their evaluation. The first table (p. 214) dichotomize authors as internal and external; the former operating directly within the case company (e.g. employees), and the latter operating indirectly. In this first table, the authors also identify the level in the organization and the specific area of contribution related to each actor. M oreover, the second table presents the assessment of each actor's power and status by characterizing these as 'high', 'low', 'average', and also level between these such as 'high $\rightarrow$ low' or 'low $\rightarrow$ average'. These characterizations of power and status are presented together with the reasons for each assessment (p. 218). Furthermore, the authors use a third table to summarize the roles of each actor in the implementation of MASs (p. 219). One of the important conclusions of the Länsiluoto and Järvenpää study is that the role and the status of an actor can change even if the degree of power remains static during the implementation of a MAS. As the reader will note, this conclusion will be supported by the findings of this dissertation when analyzing the illustrative case study of Kopla. 
In addition, Länsiluoto and Järvenpää (2010) note that their study "showed the importance of taking into consideration organizational culture [...] when implementing different management systems" (Länsiluoto and Järvenpää 2010, p. 221). Therefore, light is shed once again on the importance of arguing for the consideration of cost consciousness as a feature of organizational culture and its relationship to MASs.

Finally, I would like to close this discussion with a curiosity. When discussing the characteristics of the actors involved with the case company, Länsiluoto and Järvenpää (2010, p. 209) mention that one "of the previous CEO's roles was to introduce a profit-oriented culture, particularly the aspect of being highly cost-conscious, and promote it throughout the organization [...] The new CEO [...] continued to emphasize profit orientation and to highlight the revenue side of profit consciousness".

Busco and Scapens: The BSF, M anagement Accounting Systems, and Organizational Culture

Recently, Busco and Scapens (2011) published a study which seems have many similarities in relation to some of this dissertation's key discussions. The purpose of their study was to "explore the nature, roles and dynamics of change of management accounting systems (MAS) [and] develop an institutional framework [drawn from the BSF] to interpret the linkages between the cognitive dynamics which characterize organisational culture (viewed as shared cognitive schemas) and the behavioural and structural modalities through which they are drawn upon and reproduced by organisational members" (Busco and Scapens 2011, p. 320).

Therefore, the work by Busco and Scapens has some important similarities with respect to this dissertation as both highlight the importance of organizational culture in management accounting studies, recognizing the relationship between MASs and organizational culture (for other works analyzing this relationship see Agbejule 2011), and the use of institutional theory and the BSF to theorize on the interactions between organizational members, MASs, institutions and organizational culture.

Furthermore, the authors construct the interpretation of culture from the works by Schein, also similarly as it was conducted in this dissertation. Moreover, Busco and Scapens also note the various similarities between Schein's work and structural and institutional theory including the duality of structure and the need which actors have in dealing with anxiety.

Moreover, one of the main objectives of Busco and Scapens (2011, p. 343) is "to develop an institutional framework which is capable of explaining how and why organisational culture evolves across time and space". Hence, as it has been argued that the institutionalization process is similar to organizational culture change, an explanation of how and why cost consciousness, as a feature of organizational culture, evolves across time and space, can provide support to the theorization exercise of this work. Figure 19 presents the framework developed by Busco and Scapens. 


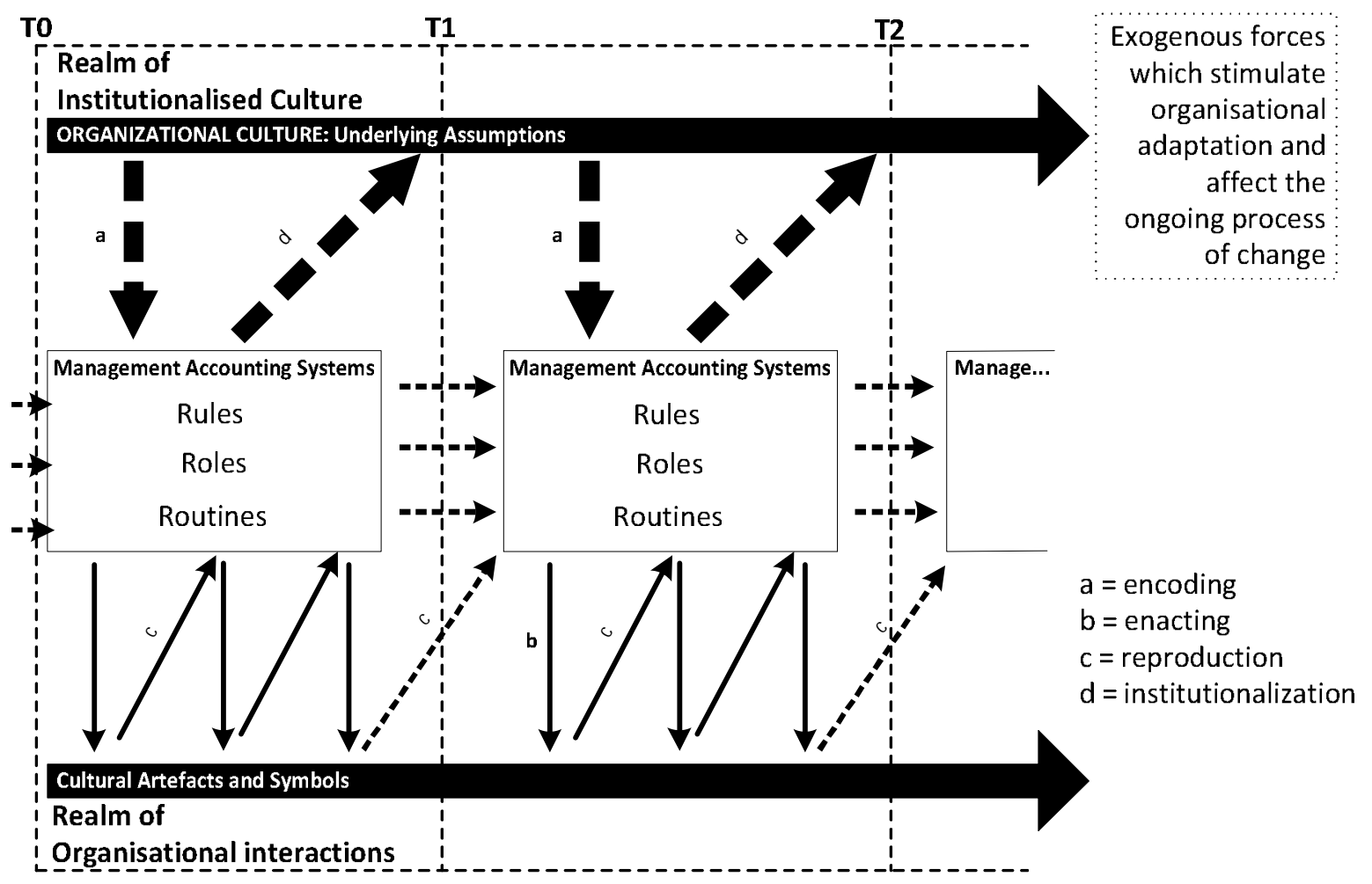

Figure 19. Evolutionary processes of change (adapted from Busco and Scapens 2011, p. 346)

There are a couple of issues with regard to the framework above which can be very relevant for this dissertation. First, Busco and Scapens (2011) argue that MASs serve as stores and carriers of cultural values, therefore highlighting the role of MAS with respect to organizational culture. Second, this framework takes into account the exogenous forces. Hence, this model allows taking into account some of the drivers associated with cost consciousness which would have been left out in the original BSF (e.g. market pressures).Third, the framework includes the differences of time which the model by Barley and Tolbert highlight, but which were left out in the original BSF. Fourth, the authors clearly depict MAS in the framework. Fifth, MAS include, apart from sets of rules and routines, the notion of roles defined as "the network of social positions" (p. 345) which, together with rules and resources are informed by the values and beliefs embodied in institutions. This addition highlights the importance of a more comprehensive analysis on the realm of action already discussed when reviewing the work by Länsiluoto and Järvenpää (2010). Moreover, the authors state that the enactment of rules, roles, and routines instantiates the essence of organizational culture in organizational interactions.

In sum, this subchapter has reviewed the origins and structure of the Burns and Scapens Framework (BSF) where various tenets of institutional and structuration theory were considered once again. The BSF allows one to analyze the processes of management accounting change which involve institutions, rules, routines, MASs, and actors. Therefore, the BSF can provide support for the theorization for the theoretical exercise of this dissertation not only because it identifies some important elements and processes which may contribute to the institutionalization of cost consciousness, but also because it allows analyzing their interaction. Moreover, this subchapter also reviewed some of the propositions, extensions, and criticisms of the BSF. Therefore, it was possible to take stock of additional elements and processes which may contribute to the theoretical exercise (e.g. the ostensive and performative aspects of routines, the power and status of organizational actors, and the acknowledgement of organizational culture as a phenomenon involved with the interaction of both intra-organizational and extraorganizational factors). 
Summarizing, this chapter has allowed an overview on Institutional Economics by first reviewing the historical path of this institutional branch since its birth on the works of Thorsten Veblen, until its reemergence in the 1970s. In addition, a review on Giddens's structuration theory has provided considerable support in clarifying important tenets of Institutional Economics. For instance, it allowed a better understanding of agency and its relationship with routines and institutions (or structures). In addition, the integration of Giddens's concept of structure with the conceptualization of institution allowed identifying five characteristics which all institutions should share. Furthermore, this chapter has also reviewed some of the differences and similarities between OIE, NIE, and NIS which are the institutional branches from where the BSF draws. Finally, the BSF was presented and reviewed together with proposals and criticisms which have been presented by various authors during the years. These proposals and criticisms will be synthesized in Chapter 5 to provide a formulation for supporting the theorization exercise. 


\section{Management Accounting, Institutionalization, and Cost Consciousness}

In this chapter I construct the theoretical exercise of this dissertation by identifying the organizational elements and mechanisms which could be involved in the institutionalization of cost consciousness; analyzing their interaction; and discussing the roles which the drivers, interpretations, and results uncovered in Chapter 3 may play in the institutionalization process. Moreover this theorization exercise will be illustrated with the use of the case study briefly overviewed in the methodological chapter of this dissertation. The illustration should serve as a guideline for the analysis of the elements and mechanism involved in the institutionalization of cost consciousness.

Hence, subchapter 5.1, drawing on the discussions held in Chapter 4, presents a formulation which includes the elements, mechanisms and forces which may be involved in the institutionalization of cost consciousness. The aim is to integrate the drivers, interpretations, and results identified and inferred in the conceptual exercise and theorize on their role. Furthermore, subchapter 5.2 describes the case study which serves as illustrative support for the theorization exercise. Finally, subchapter 5.3 integrates the formulation with the case study and, therefore, illustrates how the institutionalization of cost consciousness could be understood through an institutional and management accounting perspective.

\subsection{Integration of Conceptual Exercise in Proposed Formulation}

This subchapter aims to integrate the conceptual exercise of this dissertation into a formulation which includes the elements, mechanisms and forces that could be involved in the institutionalization of cost consciousness.

In order to take stock of these elements, mechanisms and forces; this dissertation has reviewed structuration and institutional theories with particular emphasis on the Burns and Scapens Framework (BSF). As explained, the BSF was designed to analyze the processes involved in management accounting change by framing the interaction between institutions, routines, rules and actors. Furthermore, management accounting systems (MASs) can be considered as sets of rules and routines, and, moreover, serve as modalities linking the institutional realm and the realm of action. This link, however, occurs through four main movements; encoding, enacting, reproduction and institutionalization.

Furthermore, I have also reviewed some propositions and criticisms of the BSF which could be taken into account in order to gain further insight into the elements and mechanisms which may be involved in the institutionalization of cost consciousness. Hence, Figure 20 integrates these propositions and criticisms of the BSF presented in the previous section into one framework. 


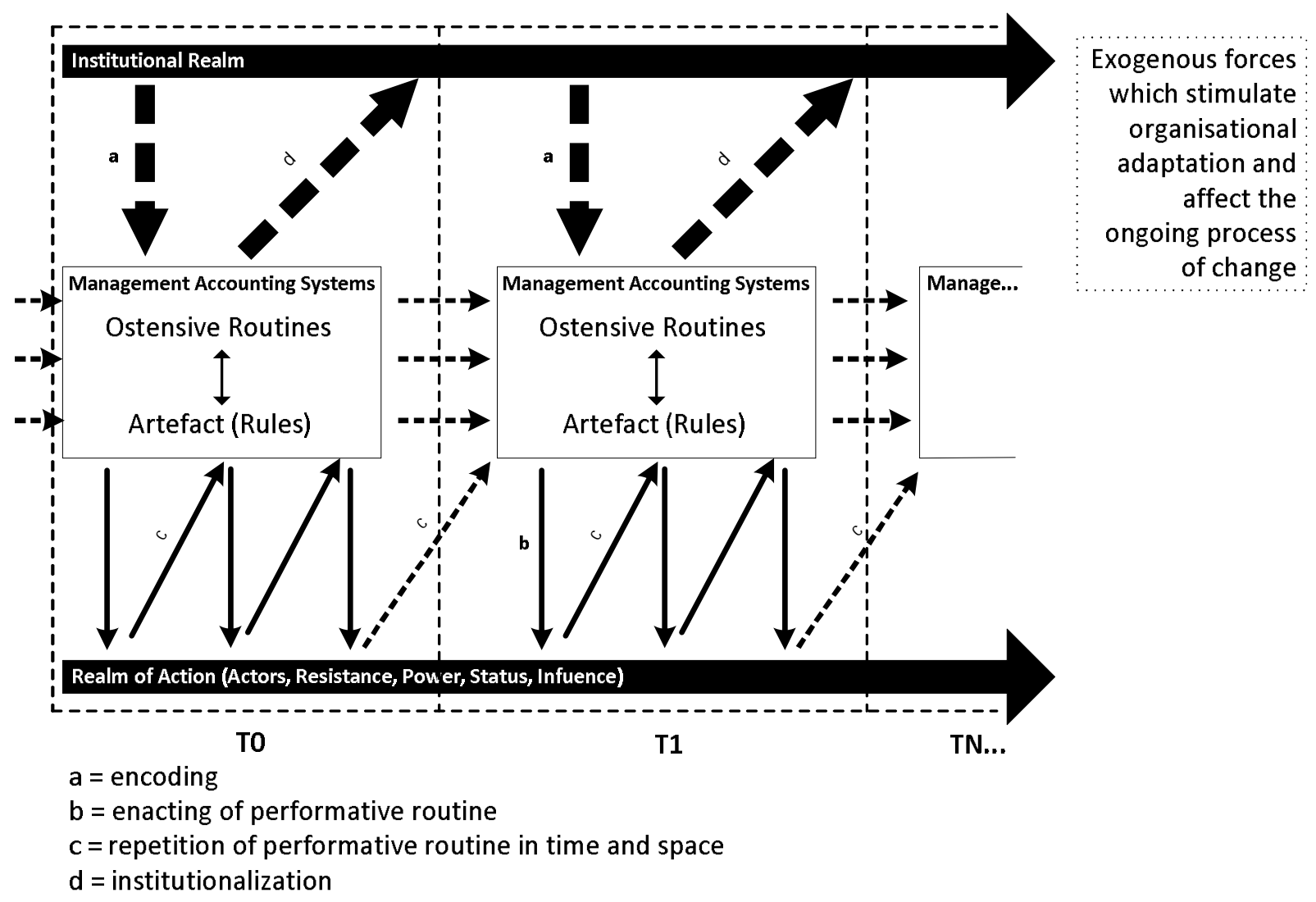

Figure 20. M anagement Accounting Systems and aspects of routines in the BSF

As the reader may observe, the figure above includes the expansion by Quinn (2011) (i.e. the relationship between the ostensive and performative aspect of routines); the discussion by Länsiluoto and Järvenpää (2010) (i.e. analyzing the roles of actors by first classifying them between internal or external, and the focusing on how their status and power change during the implementation of a MAS); and some of the contributions of Busco and Scapens (2011) (i.e. the incorporation of the exogenous forces). Clearly, this proposition can be extended as more extensions and proposals for addressing limitations of the BSF are brought forward.

Moreover, as the review on the studies that have already used the BSF noted, the synthesis and organization of these elements and processes may provide various benefits. Therefore, this dissertation proposes that the results obtained from case studies using the BSF should be synthesized, organized and summarized following the formulation depicted in Figure 21 . As the reader may note, the proposition divides the elements in five main levels: Institutional Realm, M odalities, Realm of Action, Exogenous Forces, and Change. 


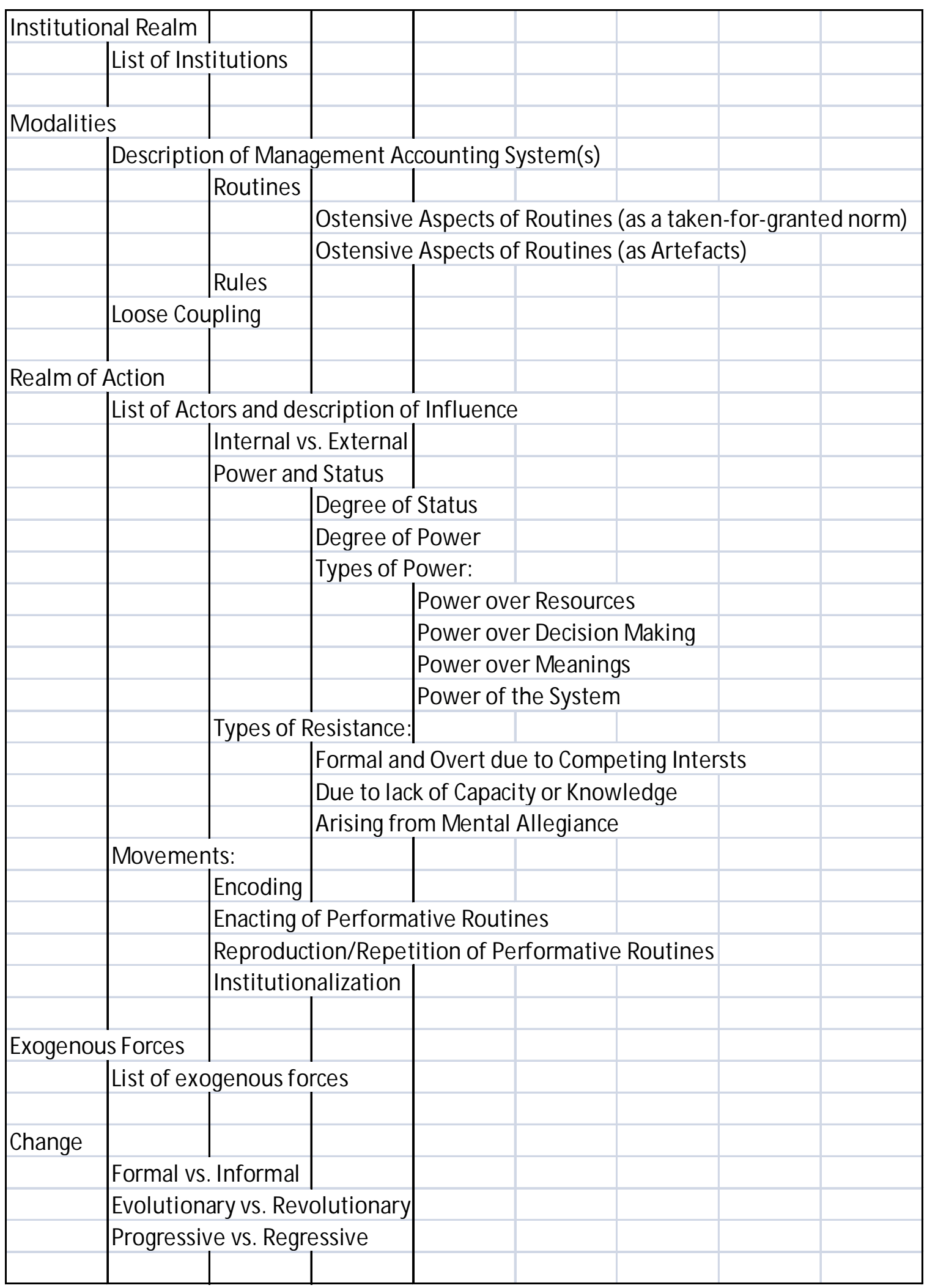

Figure 21. Formulation synthesizing relevant elements and mechanisms with respect to BSF

Hence, now it will be possible to analyze the roles which the drivers, interpretations, and results associated by management accounting scholars may play in the institutionalization of cost consciousness. In order to achieve this, the following subsections integrate the results of the literature review on cost consciousness into the formulation presented above. 
The Institutional Realm: List of Institutions

The first level concerns the Institutional Realm where the institutions prevailing in the organization reside. The only element in this level is the list of institutions which govern the actions of the individuals in the organization. The task of identifying these institutions is not simple for a couple of reasons. For example, institutions "are built up in the course of a shared history" (Berger and Luckmann 1966, p. 54); therefore, suggesting that the identification of an organizational institution might require familiarizing oneself with the history of the organization. Hence, a longitudinal study might be required in order to identify institution-candidates. Furthermore, once these institution-candidates are identified, the researcher should assess if they hold the qualities required to classify as an institution. A researcher, for example, can assess the conformity of each candidate to the features shared by all institutions previously proposed (i.e. institutions are based on shared conceptions and expectations which are taken-for-granted; institutions are intricately related to routines; institutions are stable; institutions are collective in nature; institutions provide meaning, legitimation, and power).

With respect to the results from the literature review, cost consciousness has been defined as an economic judgment of operations (Kurunmäki, 1999), a concern to contain costs (Bovier et al., 2005) or economic efficiency (Nor-Aziah and Scapens, 2007). From the interpretations inferred from the literature, cost consciousness holds in various instances a very holistic connotation involving capabilities to monitor, understand, classify, allocate and forecast costs. Moreover, the notion has also been interpreted as beliefs, which, if successfully repeated, could become basic assumptions; therefore, representing two of the three levels of an organizational culture of which cost consciousness could be a feature of. In addition, cost consciousness has also been described as an organizational culture. However, this dissertation, in an attempt to differentiate between the concepts of institution and organizational culture (thus avoiding possible conceptual discrepancies and overlaps between the vast literatures where these two concepts reside) considers cost consciousness as an institution which may represent a feature of organizational culture.

Furthermore, in practice, arguing for the institutionness of cost consciousness is a very difficult task given the difficulties in deciphering artifacts, espoused beliefs and values, and basic assumptions. Therefore, a researcher would need to spend enough time and familiarize himself/herself with the organizational members to the extent of being capable to analyze these levels of culture in a particular organization. Nevertheless, a case study studying the institutionalization of cost consciousness in an organization could approach this problem by assessing the existence of basic assumptions at the core of an organizational culture. The case study discussed in this dissertation serves this purpose as I was able to become very familiar with the main actors involved in the process of management accounting change which occurred in the case company.

\section{Modalities: Management Accounting Systems}

The second level refers to the M odalities which, from the management accounting perspective, refer to the MASs which contain sets of rules and routines. The formulation proposes that the existing MAS, before and after the implementation/modification of the MAS (i.e. T0 and T1) should be described. 
Furthermore, the reviews on the studies using the BSF suggested that the implementation and/or modification of MAS marked the beginning of the management accounting change process under scrutiny. Therefore, it is important for researchers to identify and clearly describe the MAS as it holds an important role in the process.

The synthesis of the conceptual exercise highlighted the driving role of MASs which management accounting scholars have associated with cost consciousness. Although this dissertation has taken a very broad view on MASs, it has been evident in the review of the BSF that management accounting scholars have associated the implementation and/or modification of MISs as clear markers which signal processes of management accounting change. Hence, it seems relevant to mention at this point the MISs which scholars associate as drivers of cost consciousness in an organization. These include accounting information systems (in general), casemix accounting systems, improved data systems, and product budgeting systems. Some of these MISs were associated with cost consciousness in more than one publication (e.g. budgetary systems and casemix accounting systems). Future research on cost consciousness could test if in fact these systems can serve an organization in the institutionalization of cost consciousness as it is interpreted in a particular setting.

M oreover, although this section could consider the Management Programmes identified in the conceptual exercise (e.g. ABC, Target Cost Management), it was noted that these would not be considered as drivers of cost consciousness as their causality in this respect seems to be relatively loose.

Finally, it is important that the MISs represent only some of the ways in which MASs act as drivers of cost consciousness. The use of MASs and the output of M ISs have also been considered by other authors as examples of MASs.

Modalities: Rules, Routines (Ostensive Aspect), and Loose Coupling

M AS are considered as sets of rules and routines. Furthermore, Burns and Scapens (2000) define rules as the formally recognized way in which things should be done, and routines as the way in which things are actually done or the procedures actually in use. However, given the discrepancies on the conceptualization of management accounting routines, one should refer to the ostensive aspect of the routine, both as a taken-for-granted norm and as an artifact. Feldman and Pentland (2003, p. 101) explain that the ostensive aspect of routines (i.e. the idea which shapes our perception of what the routine is) may exist as a taken-for-granted norm or as an artifact; the latter referring to, for example, a codified standard operating procedure. Hence, the proposal differentiates between these two in order to provide a more comprehensive analysis of the results.

The literature review on cost consciousness identified certain uses of MASs which were associated as drivers of cost consciousness. These include allocating costs; comparing revenues with budgets; conducting product costing; developing budgetary control establishing financial performance measures; establishing prudent budgetary goals; identifying costs and profit centers; modifying budgets, accounting and financial reports; and participating in cost budgeting. These activities could be initially set as formalized rules in a company which, after consecutive reproduction, may become routinized. Moreover, some of these activities could arise without having been previously formalized as rules. 
In addition, these particular activities could represent the performative aspect of routines as they may reflect the "actions taken by specific people at a specific time, when they are engaged in an organisational routine" (van der Steen 2011, p. 513).

Moreover, the output of AISs identified in the literature review (e.g. monthly cost variance reports, and reports on product costs) could be considered, reasonably, as ostensive aspects of routines. In addition, the performative aspects of routines previously mentioned could also result in the materialization of ostensive aspects such as reports and presentations on budgets.

Furthermore, research on management accounting could focus on analyzing if, in fact, these rules and routines could support the institutionalization of cost consciousness in a particular organization. Moreover, future research may also test other possible rules and routines which may contribute to the institutionalization of cost consciousness.

In addition, with respect to the ostensive aspect of routines; artifacts are probably easier to identify in practice when compared to taken-for-granted norms for similar reasons that explain the difficulty in identifying institutions. The case company, however, allowed identifying both rules (artifacts) and some taken for granted norms related to the routines.

Finally, the last element of this level refers to identification of loose coupling in an organization which can refer to the decoupling of rules from the daily practices in an organization. Cases of a ceremonial implementation of rules, in efforts to legitimize an organization, could arise in the institutionalization of cost consciousness. However, the literature review on cost consciousness did not offer any valuable insight in this topic

\section{The Realm of Action: List of Actors}

The third level of the formulation aims to describe the Realm of Action involved with the processes of management accounting change. The first element at this level refers to the identification and description of the actors (or group of actors) involved in the processes of management accounting change. The influence can be either direct or subtle. An example of an actor with direct influence is an IT consultant providing training for the implementation of a particular system. A subtle influence could be exerted by an accountant who resists the implementation of a new system by not attending all of the required trainings and, therefore, takes more time to adjust to its use.

The literature review on cost consciousness identified various individuals involved the processes of management accounting change (e.g. managers, IT consultants, accountants). Moreover, the results of the interviews also highlighted the role of a tough CEO in the institutionalization of cost consciousness.

In practice, the identification of these actors should be of relative ease although the quality of their descriptions could vary depending on the degree of familiarity between the researcher and the actor.

\section{The Realm of Action: Internal/External Actors and Degree of Status \& Power}

The third level also calls for the classification of each actor as internal or external with respect to the way in which she/he operates in an organization. The fact that the BSF is meant to analyze intra-organizational processes of management accounting change, does not rule out the 
possibility of including external actors who are participating in the implementation of the MAS in the organization (e.g. external consultants). As noted, this level is focused on examining if actors can be considered as internal or external to the organization, and, furthermore, the status of actors.

With respect to the internal/external relation, the literature review on cost consciousness identified many internal actors such as managers and consultants. Moreover, IT consultants could be considered as external actors. In addition, as cost of regulations was seen as factor inversely related to the driving of cost consciousness, one could reasonably argue that regulators and state legislators, for example, could also be considered as external actors associated with the processes of institutionalizing cost consciousness.

In the practical aspects, moreover, researchers should face no significant problems in differentiating between internal and external actors. The case study, however, only allowed me to familiarize myself with internal actors.

Furthermore, with respect to the degree of status (i.e. position in group's prestige hierarchy) and power (i.e. potential for obtaining favored payoffs in relations where interests are opposed) of an actor, it is worth reminding that Länsiluoto and Järvenpää (2010) assessed these by assigning to them a 'high', 'low', 'average' or intermediate (e.g. 'average $\rightarrow$ high') mark. This approach, although quite subjective as admitted by the authors (Länsiluoto and Järvenpää 2010, p. 222), provides a solution for assigning a qualitative assessment of the power of each actor.

Moreover, the conceptual exercise allows one to infer that high status and high power actors should be involved in the institutionalization of cost consciousness. As previously mentioned, a tough CEO was identified in the set of interviews as an important factor in driving organizational cost consciousness. A CEO, one could reasonably argue, is a high status and high power actor. Similarly, managers and consultants might be high status actors. However, it would be interesting to assess the power of these actors since, for example, consultants might not have real power in influencing the implementation of a particular M AS in an organization.

Finally, in practice, researchers could provide a qualitative assessment of the status of the key actors in the process. This, however, remains a highly subjective assessment, therefore easily prone to criticism.

\section{The Realm of Action: Power}

Moreover, the four categories of power introduced by Hardy (1996) are also included in the formulation. The first category of power (i.e. power of resources) is expected to be identified in many cases as it is a common form of power exercised by deploying information, expertise, credibility, prestige, control of money, rewards and sanctions. Furthermore, previously discussed, Shields and Young (1994) identified cost management knowledge as a clear determinant of cost consciousness. It might be interesting to explore how a cost management knowledgeable individual can exert power over resources to increase cost consciousness in the organization. Another possible association can be made with the results by Abernethy and Vagnoni (2004) who stated that informal authority is indirectly related to cost consciousness. Hence, as one may reasonably consider informal authority and prestige to be related, it might be possible to conduct research on how power over resources could be indirectly related to an increase of cost consciousness whenever key actors use informal authority. 
Furthermore, the second category of power (i.e. power of processes) may also take an important role in the institutionalization of cost consciousness. This type of power refers to the situations where dominant groups invoke a variety of procedures and political routines to influence outcomes by preventing subordinates from participating in decision-making. In addition, the literature review on cost consciousness allows one to make at least one association. The study of Shields and Young (1994), for example, identified cost budget participation as a determinant of cost consciousness. Therefore, it could be argued that this category of power may be used both against and in advancing the institutionalization of cost consciousness. Hence, opportunities for research also arise with respect to the evaluation of power over resources and its effect on the institutionalization of cost consciousness.

Moreover, the third category of power (i.e. power over meaning) occurs when symbols, rituals, or language are used to shape perceptions, cognitions and preferences in order to make change appear legitimate, desirable, rational or inevitable. Furthermore, interestingly, these symbols, rituals, and language are part of the artifacts representing the most visible level of culture in an organization. Hence, the indirect relationship between informal authority and cost consciousness could also be related to this kind of power as actors with informal authority may have the capacity to shape perceptions or preferences.

In addition, the fourth category of power (i.e. power of the system) refers to the type of power which is taken for granted and is deeply embedded within organizational systems. The literature review identified certain possible espoused beliefs associated to cost consciousness which, if successfully repeated, could become basic assumptions and reflect the core of an organizational culture which could be composed of cost consciousness as an institution. However, it was not possible to associate any particular finding with this type of power.

Finally, in practice, considerable familiarization with an organization and its members might be necessary in order to identify these types of power in a case study.

\section{The Realm of Action: Resistance}

The analysis of the Realm of Action, as proposed in this dissertation, also includes the three types of resistance identified by Burns and Scapens (2000, p.17). The first type of resistance (i.e. formal and overt due to competing interests) finds associations with respect to the results of the literature review as an indirect relationship between informal authority and an increase of cost consciousness was identified. In a case of competing interest, informal authority may play an important role by allowing the movement of resources to exert power. Hence, formal resistance - deployed through informal authority - may be detrimental to efforts on the institutionalization of cost consciousness.

Furthermore, the second type of resistance (i.e. actors not having the capacity or knowledge to implement the change) finds support from the literature review as cost management knowledge has been associated as a driver of cost consciousness. Therefore, there might be an indirect relationship between this type of resistance and an increase of cost consciousness.

The third type of resistance arises from mental allegiance to specific ways of thinking and doing in the organization. Unfortunately, the literature review on cost consciousness does not allow any insight on this type of resistance. 
The Realm of Action: The Four M ovements

The final elements of the third level of the proposition refers to the four movements of the BSF (i.e. encoding, enacting of performative aspect, repetition of performative aspect, and institutionalization) should be described. The first movement refers to the encoding of the prevailing institutional principles in order to shape new rules and form and/or reform the ongoing routines. Furthermore, the second movement refers to the enactment of the performative aspect of routines (and/or rules) either consciously or unconsciously. This leads to the reproduction the performative aspect of routines (and/or rules) are reproduced involving, at the same time, either conscious or unconscious change. Finally, if these rules and routines become simply the way things are, then they can be said to have become institutionalized.

Moreover, the performative aspect of a routine is the actual performance of the routine best understood as being inherently improvisational. Furthermore, as mentioned, the uses of MASs which management accounting scholars have associated with cost consciousness (as drivers) could represent the performative aspect of routines. Hence, there is an association between the enactment and reproduction movements with the findings of the literature review on cost consciousness. However, it is not possible to make a reasonable association between the findings and the encoding and institutionalization movements.

In practice, researchers should be able to identify the performative aspect of routines in retrospective. However, the encoding and institutionalizing movements are probably more difficult to describe as they involve the basic assumptions and taken-for-granted ways of thinking in the organization. Particularly, describing who conducts these movements might require a considerable amount of subjective reasoning.

\section{Exogenous Forces}

The fourth level of the model relates to exogenous forces which may influence the ongoing processes of change. When incorporating these into their framework, Busco and Scapens (2011) stated financial market as an example of such forces. The inclusion of exogenous forces in the analysis of the BSF provides a more holistic view which is not restricted to the intraorganizational processes.

As noted, the inclusion of these forces into the BSF is welcomed by this dissertation as the results of the literature review identified market pressures, a market crash, economic depressions, and industry deregulations as drivers which management accounting scholars have associated with cost consciousness.

\section{Change}

The fifth level of the model reviews change by exploring the three dichotomies of change highlighted by Burns and Scapens (2000, p. 18). The first dichotomy of change (i.e. informal vs. formal) already has plenty of possibilities set out by the literature review conducted in this dissertation. For example, the MAS identified as possible drivers of cost consciousness (mentioned above), if implemented or intentionally modified, would clearly reflect cases of formal change. Moreover, if the improvement of data systems, reforms of financial management reforms, changes in budgets, accounting, and financial reports (which have also been identified as possible drivers of cost consciousness) would occur at a tacit level (i.e. somehow 
unintentionally, perhaps resulting from adaptations to changes), then they would be examples of informal change. However, although in theory it seems simple to classify change as either formal or informal; Burns and Scapens (2000, p. 19) note that studies "of formally planned changes in management accounting systems are likely to be easy to conduct, while studies of informal processes of change are likely to be much more difficult".

The second dichotomy of change (i.e. revolutionary vs. evolutionary) also finds support from the results the conceptual exercise as, for example, the reforms and improvements to MASs and MISs could also represent potential examples of evolutionary change if they are conducted gradually. However, the implementation of these MASs in environments which did not previously use that particular system (or a similar one) could draw a case of revolutionary change. Within this respect, Burns and Scapens (2000, p. 20) note that is important for researchers to acknowledge that while an apparently minor management accounting change may result in major institutional consequences, a rather significant management accounting change may lead to only limited impact.

The third dichotomy of change (i.e. regressive vs. progressive) can also be related to the results obtained from the literature review as informal authority may indeed result in regressive change if, for example, it is used to resist the implementation of a M AS. However, the implementation or modification of the previously identified MAS can eventually result in a case of progressive change if these ultimately serve the organization in a positive manner.

In sum, this subchapter has presented associations between the synthesis of the conceptual exercise and the organizational elements (including processes and forces) which could contribute to the institutionalization of cost consciousness. With respect to the first level of the proposition (i.e. the Institutional Realm), the theoretical exercise associated the interpretations of cost consciousness as a feature of organizational. Furthermore, the drivers that management accounting scholars have associated with cost consciousness could play role in the second level of the proposition as MASs, rules, and routines (with respect to both ostensive and performative aspects). M oreover, some of these drivers could also be involved in power of resources, power of processes, power over meaning, formal and overt resistance due to competing interests, and resistance related to actors not having the capacity or knowledge to implement the change. In addition, the theoretical exercise also associated some drivers of cost consciousness as possible exogenous forces involved in the institutionalization process. Also, while MISs have been associated with formal and revolutionary change, informal authority has been associated as a possible case of regressive change. In addition, it would also be interesting for future research to focus on the elements of the proposal which found no associations with the results of the literature review (e.g. power of the system, resistance related to mental allegiance to specific ways of thinking and doing in the organization).

Although this dissertation is mainly of a conceptual and theoretical nature, a case study serves as an illustration of how the formulation may serve the analysis of an organization where cost consciousness is intended to be institutionalized by implementing a very basic MAS. This case study will be described is described in the following subchapter. 


\subsection{Description of Case Study: A Colombian Food Processing Company}

The case study analyzed in this dissertation describes the efforts of a company's General Director (who is also the major owner of the company) to institutionalize cost consciousness in its organization. The General Director (GD) wanted to change the previous organizational culture which was, on his own words, "too relaxed" and "not-business oriented enough". In other words, the GD wanted to de-institutionalize a non-business oriented culture by institutionalizing cost consciousness. Together with certain organizational measures (e.g. modifying the organizational structure), the GD decided to implement a simple MAS during the first semester of the year 2011. Research for this case study took place over a period of four years; from 2008 to 2012.

The brief description of the case study above already sets forward possibilities for analysis as it suggests that cost consciousness is expected to be enough business oriented. However, following the discussions in the previous chapters, it may also be that cost consciousness may support business orientation in the composition of a particular organizational culture; one which the GD of Kopla pursues.

\section{Overview of Kopla}

Kopla is one of the brands of Punto Franco Agrocommodities (PFA), a small agribusiness company focusing on planting, harvesting, processing, and marketing of various food products. Although PFA owns various businesses and brands, this case study focused solely on the activities concerning Kopla. The main offices of Kopla are located in Armenia, a small-medium sized city in Colombian standards (population of $292045^{45}$ ) situated in the central Andean region of the country. Armenia is the capital of Quindío (one of Colombia's thirty-two departments ${ }^{46}$ ) which is considered to be one of the three departments forming Colombia's Coffee Axis, the other two referring to Risaralda and Caldas. Furthermore, apart from the coffee production, the region's climate and topography allows for the harvesting of various crops including plantain, avocado, sorghum, potato, cassava, corn and sugarcane; all of these being common in the diet of Colombians and people in neighboring countries.

\section{Kopla's Raw Materials, Final Products, and Customers}

The processing plant where the Kopla branded products are manufactured is located a few kilometers outside the city with convenient access to main roads connecting to other regions including Bogotá, the country's capital. Moreover, the processing plant is responsible for receiving four basic food products (cassava, plantain, potato, and arracacha ${ }^{47}$ ) and processing these into different finished and semi-finished food products (e.g. shredded cassava, peeled and

\footnotetext{
${ }^{45}$ According to data obtained from DANE (National Administrative Department of Statistics) on the January $30^{\text {th }} 2012$ from their website:

http://www.dane.gov.co/index.php?option=com_content\&view =article\&id=75\&/temid=72

${ }^{46}$ Referring to the administrative and political subdivisions of the country as in other Latin American countries (e.g. Argentina, Bolivia, Uruguay) and, also, in France.

${ }^{47}$ Arracacha (arracacia xanthorriza) is a perennial plant originally domesticated in South America and currently cultivated mainly across South and Central America, the Caribbean. The principal economic product of the arracacha are its storage roots which are used for culinary purposes and can be considered as a staple food in many countries (Hermann, 1997)
} 
cut plantain, potato cut as french-fries, frozen arracacha cut in pieces). Figure 22 provides an overview of the raw materials and their relationship to the final products of the company.

\begin{tabular}{|c|c|}
\hline Raw Material & Final Product \\
\hline Cassava (First class) & Cassava: Raw, Pealed, in Pieces, Frozen \\
\hline Cassava (Second class) & Cassava:Pealed, in Pieces, Pre-cooked, Frozen \\
\hline Cassava (Third class) & Cassava:Shredded \\
\hline Plantain (Un-ripened, First Class) & Cassava:French-cuts, Pre-cooked \\
\hline Plantain (Un-Ripened, Second Class) & Cassava:Croquettes, Pre-fried, Frozen \\
\hline Plantain (Un-Ripened, Third Class) & Plantain:Un-ripened, Patacones \\
\hline Plantain (Ripened, First Class) & Plantain: Ripened, Patacones \\
\hline Plantain (Ripened, Second Class) & Plantain:Un-ripened, French-cuts \\
\hline Plantain (Ripened, Third Class) & Plantain: Ripened, French-cuts \\
\hline Arracacha (White, First Class) & Arracacha: Raw, in Pieces, Frozen \\
\hline Arracacha (White, Second Class) & Potatoes:French-cuts \\
\hline Arracacha (Yellow) & \\
\hline Potato (Imported) & \\
\hline
\end{tabular}

Figure 22. Illustration of Kopla's raw materials and final processed products ${ }^{48}$

As it can be observed, the final products of the company are relatively simple. However, it might be helpful to provide the clarification of two concepts. First, French-cut refers to a cut similar to the typical French-fries (i.e. narrow, elongated and in somehow cubical forms). Second, patacones refers to a process where the plantain is cut into pieces, then fried, and finally each piece is pressed to form circular and relatively thin pieces as it can be observed in the process of making plantain ripened patacones (Figure 23).

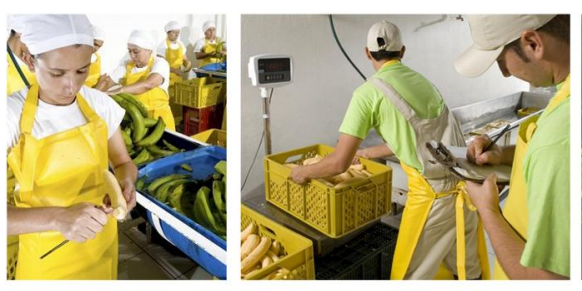

Peeling
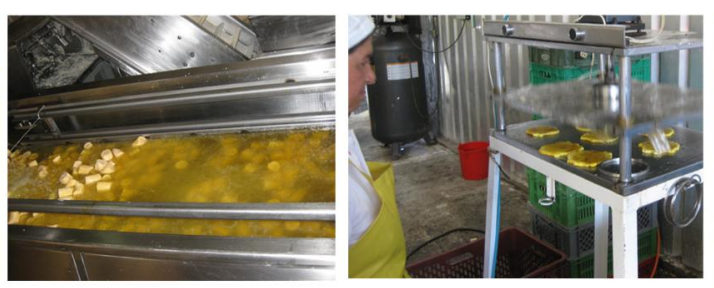

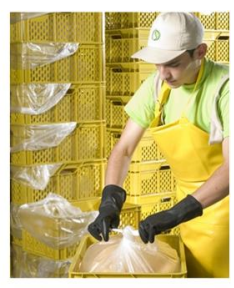

Packing

Figure 23. Process of making plantain ripened patacones ${ }^{49}$

It should be noted that, not all the steps of the process are shown in the figure above including the washing (after peeling) and final packaging and refrigeration. However, the process, as it can be observed in the figure above, is carried out in a considerable manual way with limited automatization.

Moreover, the various products are sold to customers whom the company categorizes into three main groups:

A. Supermarkets. As it name suggests, these clients include all supermarkets and stores inside the city of Armenia.

B. Institutional. Institutional clients include hotels and restaurants inside the city of Armenia

\footnotetext{
${ }^{48}$ Photographs reproduced with permission of Kopla

${ }^{49}$ Photographs reproduced with permission of Kopla
} 
C. Outside Armenia. This type of clients include all of those situated outside of the city of Armenia, including one big multinational company which further processes some of Kopla's products into finished goods (e.g. cassava chip bags). Furthermore, large Colombian supermarket chains operating in many locations in the country are also included in this type.

Moreover, the company sells these products to other businesses and end-consumers through the following distribution channels:

- Selling products to other businesses which process them further (e.g. into bagged cassava chips)

- Selling the products with the Kopla brand in large and medium sized market chains

- Selling the products in large market chains with the market chains' bran

- Selling the products to individual consumers in their offices

As the company produces products that represent staple food in the country, the competition is considerable. According to the GD, the company has two competitors with plants nearby. Moreover, the nature of the business presents difficulties for the company's cost evaluation as the value of the raw materials (i.e. plantain, cassava, potato and arracacha) varies significantly during the year. Although some periodical patterns corresponding to price-change exist, it is not uncommon for these to experience variations. This is only one of the reasons explaining the difficulty in raising cost consciousness inside Kopla. However, the company's management has pursued (both intentionally and unintentionally) to increase the cost consciousness in the company for improving decision making processes.

\section{Size and Structure}

The company's Balance Sheet Total in December $31^{\text {st }} 2010$ was $\$ 1.2$ billion Colombian Pesos (COP) (around 481,000 Euro ${ }^{50}$ ). Furthermore, with respect to number of employees, Kopla employs nine full time employees in its office and plant, and around fifty part-time workers in the plant. Given the number of employees, and Balance Sheet Total, it is possible to classify the case company as a small enterprise with the use of the parameters established by the Colombian Legislation (Table 12).

Table 12. Company size according to Colombian Legislation and European Commission ${ }^{51}$

\begin{tabular}{|l|c|c|c|c|}
\hline & \multicolumn{2}{|c|}{ Colombian Legislation } & \multicolumn{2}{c|}{ European Commission } \\
\hline & $\begin{array}{c}\text { Number of } \\
\text { employees }\end{array}$ & $\begin{array}{c}\text { Balance Sheet Total } \\
\left(\text { SM M LV }^{2}\right)\end{array}$ & $\begin{array}{c}\text { Number of } \\
\text { employees }\end{array}$ & Balance Sheet Total \\
\hline Micro & $0-10$ & $\leq 500$ & $0-9$ & $\leq 2 \mathrm{~m} €$ \\
\hline Small & $11-50$ & $500-5000$ & $10-49$ & $\leq 10 \mathrm{~m} €$ \\
\hline Medium & $51-200$ & $5000-30000$ & $50-249$ & $\leq 43 \mathrm{~m} €$ \\
\hline Large & $\geq 201$ & $>30000$ & $\geq 250$ & $>43 \mathrm{~m} €$ \\
\hline
\end{tabular}

\footnotetext{
${ }^{50}$ Using the currency exchange of 1 Euro to 2495,31 Colombian Pesos (COP) valid on July $19^{\text {th }}, 2011$

${ }^{51}$ Information obtained from Bancoldex (Banco de Comercio Exterior de Colombia) on July 19th 2011: http://www.bancoldex.com/contenido/ contenido.aspx?conlD=315\&catID =112

${ }^{52}$ The SM M LV for the year 2011 was 535600 COP (approx. 214.84€ on July $19^{\text {th }}$ 2011). SM M LV stands for

Salario M ínimo M ensual Vigente and represents the minimum monthly salary in Colombia.
} 
Our case company is classified as small under both definitions in terms of number of employees. However, in terms of Balance Sheet Total, while it is classified as small under Colombian Legislation, it is considered as a microenterprise by the definition of the European Commission.

Furthermore, the organizational structure of Kopla depicted in Figure 24 reflects the steep hierarchical structure managed inside the company on January 2012.

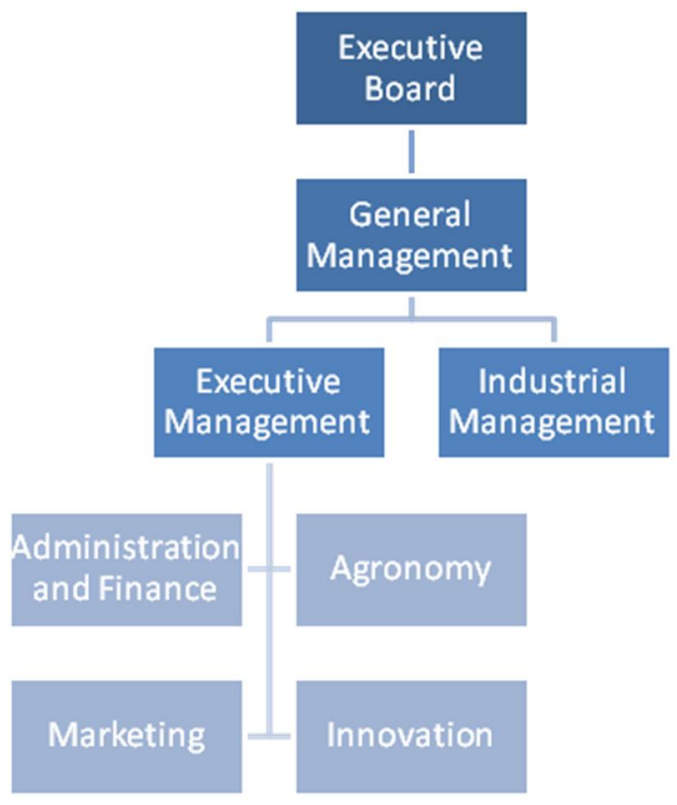

Figure 24. Kopla's organizational structure in the year 2012

The Executive Board (i.e. Board of Directors) is composed by the GD, the General Manager (GM) who is in charge of the General Management unit, the Industrial M anager (IM) in charge of the Industrial $M$ anagement unit, the Financial $M$ anager (FM), the Agronomy $M$ anager, the $M$ arketing Manager, and the Innovation Manager (InnM), each in charge of the respective sub-units. Moreover, most of my interactions, meetings, and interviews were held together with these Board-members, especially with the GD and the IM.

However, it should be noted that this hierarchical structure has been modified during the last few years. At the moment in which I became involved in this case study the Executive Management did not exist and the General Management was in charge of the five sub-units (the four sub-units which are now controlled by the Executive Management together with the Industrial Management function). Furthermore, considerable changes in the personnel have taken place. On the one hand, some employees who were present at the beginning of the case study have left the company and others have been hired. On the other hand, some employees have been promoted during the last years to higher positions. These changes will be described in the following sections.

\section{Kopla's Financial Performance}

In terms of Income Statement figures, Kopla has experienced considerable variation especially with respect to its Revenue which peaked on 2009 to 3.4 billion COP (approx. 1.4 million Euro), but then experienced a decline of almost 50\% in 2010. Furthermore, during 2009 and 2010, the company has experienced financial loss reporting an Operating Profit of -280 million COP in 2010 (approx. 112 thousand Euro). These financial results are depicted in Figure 25. 


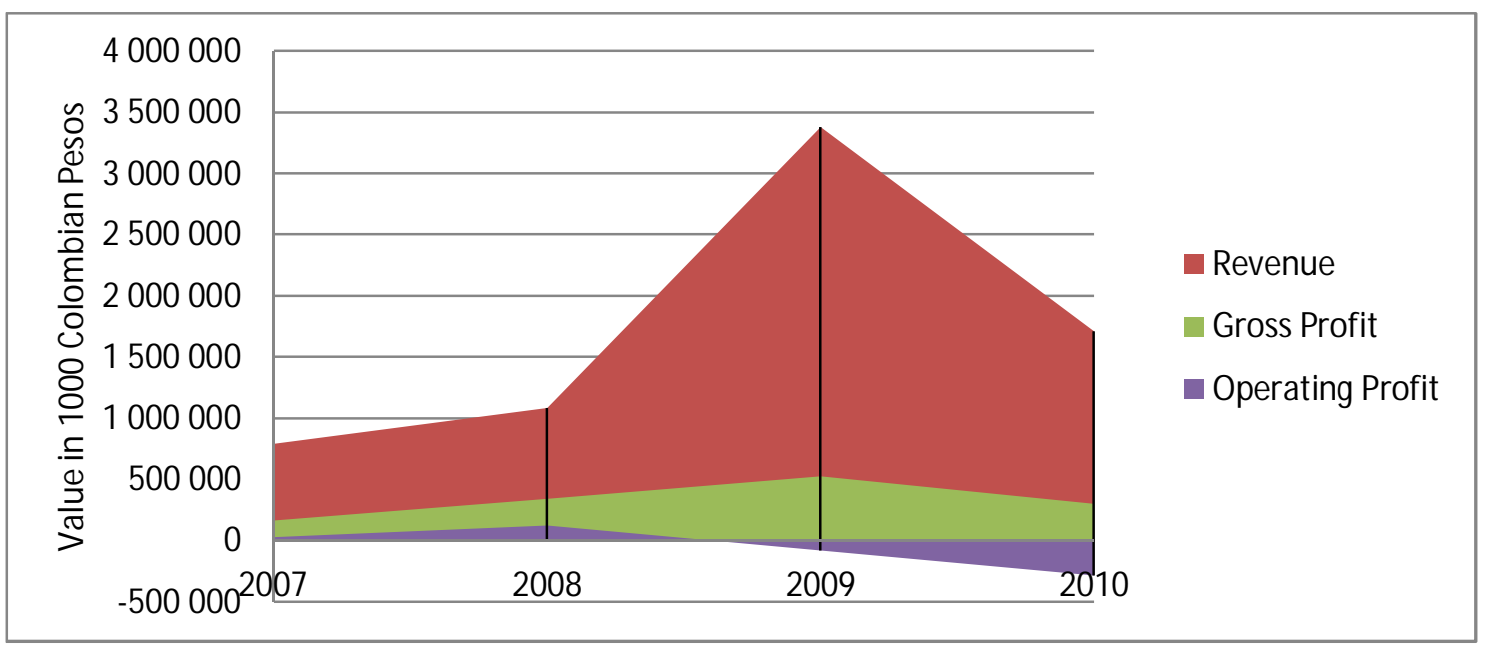

Figure 25. Revenue, Gross Profit and Operating Profit of Kopla from 2007-2010

With respect to cost structure, Figure 26 illustrates the major cost categories and Net Profit as percentages with respect to total income.

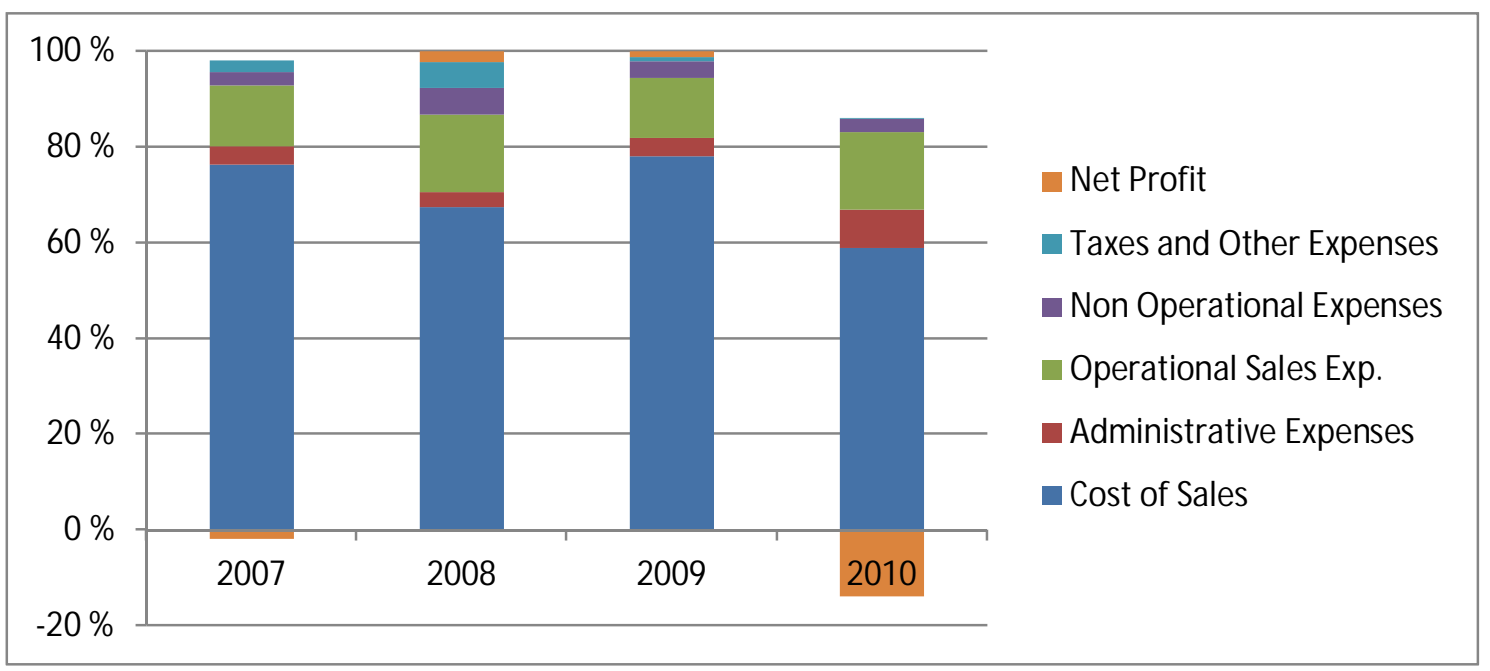

Figure 26. Cost structure of Kopla from 2007-2010

The Cost of Sales clearly represents the largest group of expenses followed by Operational Sales. The most significant changes in the cost structure of the company are reflected in a higher percentage of Administrative Expenses, particularly in the year 2010, which were one of the main reasons for the decrease in profit which lead to a loss on 2010. It should be noted that, however, that this company is not necessarily interested in maximizing its Income Statement profit. As Fridson and Alvarez (2002, pp. 99-100) note: "a publicly owned company generally seeks to maximize its reported net income, which investors use as basis for valuing its shares [...] In contrast, a privately held company has no public shareholders to impress [and] typically prepares one set of statements, with the tax authorities foremost in its thinking. Its incentive is not to maximize, but to minimize, the income it reports, thereby minimizing its tax bill as well".

\section{Kopla before the introduction of the management accounting system}

I had the opportunity to visit the company's headquarters and plant on three occasions before the introduction of the MAS in the company. On the first visit on 2008, I had the chance to interview five members of the organization: the General Director and owner (GD); the General 
Manager (GM 1); the Quality Control Manager (QCM); the Plant Engineer (PE); and one accountant $(A)$.

The first contact which I had with the company was with the GD who did not hesitate to show his big dreams about the company:

"The previous owner was known to be a very good and caring man. However, when he died his children did not want to run this company so they sold it [...] I see a lot of potential for the company but things will have to change [...] we have to think big" - GD (interview on 2008)

In fact, by this time, only a few months after buying the company, the GD had already incurred in considerable marketing and advertising expenses for a new company logo and website. Moreover, the GD was the owner of a farm which would supply Kopla with a considerable amount of cassava and plantain.

The differences between the old and new owner were shared by other members of the company. The General Manager (who also was the General Manager under the previous administration) said on that occasion:

"The situation with the previous owner was good although the company was never very profitable. In that respect it is clear that the situation with [the new GD] is different. He has been very clear in letting us know that this is a business". - General Manager 1 (interviewed on 2008)

In a way, it could be said that the change in ownership triggered both a need for providing results and the capacity to suggest new ideas. The Plant Engineer, for example, expressed this on my first interview with him:

"I have good ideas. For example, I think that we should have a truck-weighting equipment inside the company in order to avoid having to weight the incoming trucks outside the plant. M aybe you could tell this to [the GD]?" - Plant Engineer (interviewed on 2008)

This, moreover, suggested to me that the employees of the company saw in me a channel for expressing their wishes. Perhaps, although there was now a need for delivering results, it was not clear how to deliver the ideas to the higher managing positions. My personal perception of Colombian culture tells me that the hierarchical structure in Colombia is considerably steeper when compared to countries like Finland. In Colombia, it is not as easy, as it is in Finland, to simply knock on the door of a superior. This might explain why the Plant Engineer asked me to pass on his message.

Furthermore, with respect to the monitoring and control of costs, I asked the GD about costs and about any possible costing techniques that they used:

"We have many excel files filled with cost information. [...] I am not sure about the technical name of the costing technique, but [the accountant] will show you how it is all being done now". - GD (interview on 2008)

However, the accountant told me that her responsibility was mainly to introduce the cost figures of the plant and payroll into their system and worksheets. Therefore, I asked the General Manager about what they did with the costs from a management accounting perspective. 
"We practically need this information only for financial reporting. However, when one particular cost, for example natural gas, climbs, we take notice" - General Manager 1 (interviewed on 2008)

It was reasonably clear at this point that the use of cost information was limited to Financial Statement reporting and to spot irregular events. The GD took notice of this:

"I am aware that we have plenty of work to do on this respect. If it were up to me, I would like to know how much it costs us to produce each bag of patacones [...] It is necessary to bring someone who knows about this into the company". - GD (interview on 2008)

The second visit to Kopla and the corresponding set of interviews took place on 2009. On this occasion I took part of a board meeting which was attended by the General Director and owner (GD); the Quality Control Manager; the Plant Engineer; the new Financial Manager; and the administrator of the farm owned by the GD. On the way to the board meeting, the GD told me:

"As you will see things are changing here. However, there is still much to be done. Today I want to see who stays and who has to go". - GD (interview on 2009)

When I asked him how things were developing with the General Manager, he answered:

"She does not work with us anymore". - GD (interview on 2009)

According to the GD, the General Manager was fired because the company did not achieve the financial results which were expected.

The board meeting was held in one of Armenia's high-end hotels. The meeting began with the GD introducing the attendants and then asking everyone to write down the company's vision and mission on a piece of paper. Then, the GD collected the papers and said:

"If we want to make this a business, we have to have clear where we want to be not only this year and next year, but also ten years from now" - GD (board meeting on 2009)

After this, each one of the other attendants to the meeting (except me) presented different issues concerning the company. The new Financial Manager, for example, analyzed some annual financial figures with the use of simple financial ratios such as the Current Ratio and the Debt to Equity ratio. Moreover, during the meeting, the GD made his point clear:

"It is important for everyone sitting here to be aware about what is happening in the company, not only in your own divisions. [The Quality Manager] has to be aware about what [the Financial Manager] knows and the same goes the other way around." - GD (board meeting on 2009)

During a break in the meeting I asked the Plant Manager if he understood what the Financial M anager spoke of. His answer was clear:

"I need to learn more about finance because she was talking in Chinese to me" - Plant M anager (board meeting on 2009)

After the meeting was over, the GD said in private:

“Today was [the Financial Manager's] opportunity to show off. She didn't take it. I think I need someone else". - GD (interview on 2009) 
On the third visit to the company 2010, the Financial Manager position was available. Moreover, the Quality Control M anager had been promoted to General M anager (referred from now on as General Manager 2 (GM2) to avoid confusion with previous) and the GD did not save any words to express his content with her:

"She is really great. I always knew that [she] would become the General Manager, but she needed time to grow. With time she showed me that she was ready and now I am really satisfied with her work and with the company in general [...] We are opening the business and Success [this is a fictional name referring to one of Colombia's biggest supermarket and retail chains] has agreed to distribute some of our products". - GD (interview on 2010)

Furthermore, when interviewing the newly assigned General Manager 2, she said:

"The business is running ok. We now have big customers which mean big responsibilities. Hopefully we can manage them [...] It is not easy. But I will do my best". - General Manager 2 (interview on 2010)

In addition, with respect to costs, the General Manager said:

"[The GD] has made it clear that costs are very important. We have a lot of information and I am trying to organize it in order to use it better" - General Manager 2 (interview on 2010)

Moreover, the Plant Engineer had been promoted to the position of Plant Manager and a new Financial Manager had been put in place. The company did show signs of improvements. For example, I ran into two trucks carrying the logo of the company in the city, something that did not exist before as they had very recently invested in vehicles to transport their products to small institutional customers.

\section{Kopla's new management accounting system}

The case company works with very simple accounting systems. Most of the information is stored and analyzed in the Microsoft Excel spreadsheet files which the accountants fill in a periodical basis. Moreover, on the first semester of 2011, the GD introduced a tool whose purpose was to enhance the tracking, monitoring and analysis of the costs incurred by the company. The tool is an Excel file with twenty-four worksheets:

1. Index. Table of Contents for the complete Excel file.

2. Product Processes. Matrix linking the eleven final products with the 34 possible processes (e.g. peeling, cooking, and freezing) which each raw material could go through in order to be converted into each product.

3. Raw Material Entrance. Matrix presenting a relationship between the eleven final products and the type and quantity of raw material which are used to produce each final product.

4. Raw Material Prices. Matrix linking the thirteen types of raw materials which the company purchases and calculates the total cost of purchase by multiplying the price per kilo of fresh raw material times the amount (kilos) of raw material purchased

5. Adapted Raw Material. As an important weight of fresh raw material is discarded after washing and peeling, this worksheet compares the amount of raw material which was 
originally purchased (in kilos) with the amount of raw material which is left for production after washing and peeling.

6. Cost of Peeling per kilo. Calculation of the total cost of peeling the fresh raw materials by presenting a matrix which links the thirteen types of raw materials with the cost of peeling each type (calculated by multiplying the price per kilo of peeled raw material and the total amount of peeled kilos of each raw material).

7. Time and Value Day/Worker. Matrix presenting a relationship between each final product and the cost per worker. In other words, it summarizes the labour costs in producing each raw material by multiplying the amount of hours needed to complete each final product with the cost per hour of that product.

8. Final weight. Summary of the weight of each final product at the end of the process and compares it with the values in the 'Adapted Raw Material' worksheet.

9. Final weight after packaging. Summary of the weight of each final product after packaging and compares it with the values in the 'Final weight' worksheet.

10. Shifts in month. Matrix comparing the eleven final products and the amount of monthly work shifts in the production .

11. Cost of white collar workers. Calculation the total amount of payroll expenses by listing the full time workers and their respective salaries and compensations.

12. Natural Gas. Matrix linking the eleven final products and the monthly natural gas expenses allocated to each product.

13. Frying Oil. Matrix presenting a relationship between the eleven final products and the monthly frying oil expenses allocated to each product.

14. Salt. Matrix linking each of the eleven final products with the monthly salt expenses allocated to each product.

15. Packaging. Summary of the packaging costs for the final products

16. Maintenance. Summary of the maintenance costs of fourteen fixed assets (e.g. mill, filters, industrial thermometers, vehicles, computers).

17. Utility Services. Summary of the expenses related to thirteen utility services including internet, sewer, telephone and cleaning services

18. Other Costs. Summary of eighteen 'other costs' which include industrial paper, transportation costs of personnel, and vehicle repair.

19. Agrocommodities billing. Summary of the bills related to Agrocommodities.

20. Sent merchandise. Summary of the freight prices to four different cities.

21. Monthly turnover. Summary of the sales on the month of May 2011 of each final product.

22. Raw material control. A log tracking the kilos of each type of raw material

23. Shift beginning-end. Summary of the costs per shift of each of the eleven final products.

24. Margin prices. Summary of the profit margins of each of the eleven final products. There are actually two different matrixes which divide the information with respect to national and international clients.

The twenty four worksheets which compose the tool introduced by the GD have various purposes. First, they allow calculations of product, labour and overhead costs. In this way, the GM 2 is capable of classifying these costs and assess which are increasing with respect to previous months. Moreover, these figures allow the comparison with product revenues, thus assessing product profitability. Second the tool also allows monitoring the quantity of raw material which has been used in the production; therefore allowing the GM 2 and the Plant Manager to assess the amount of material needed in the future and minimize inventory. In addition, these 
capabilities also allow the managers to be better prepared against their competitors as they have access to information which was not previously available to them.

Therefore, taking into account the purposes of MASs already discussed (i.e. provide accurate product cost information, derive the most profitable product mix decisions, minimize inventory, adopt and implement plans in response to their competitive environment), it is possible to reasonably argue that the tool introduced by the GD represents a type of MAS.

The system was not given a particular name and the Board members refer to it simply as "La Herramienta de Costos" which translates to "The Cost Tool". One separate Excel file is created for each month so that the name of the file is simply 'COSTOS KOPLA (Month)' which, for the case of the month of October, would translate into COSTS KOPLA OCTOBER. Furthermore, upon the implementation of the system, the task of updating the information was assigned to two employees, namely the Industrial M anager (IM) and the Innovation Assistant (InnA).

\section{Kopla after the introduction of the management accounting system}

The management accounting tool was developed by the General Director of the company. I attended a Board M eeting on the June 2011 where the tool was reviewed by the Board M embers which included the General Manager, the new Financial Manager, the Plant Manager, and the Public Relations M anager. This presentation was held on a restaurant a few kilometers from the plant.

The management accounting tool was still in process of development. In fact, during the meeting, the GD was providing instructions about how to improve the tool. Most of the instructions seemed to be directed to more aesthetic issues such as titles and headings. However, the GD also highlighted other more technical issues including making sure that a particular figure (e.g. kilos of incoming prime cassava) should be introduced to the worksheet in only one cell and if other worksheets needed that figure then it should be recalled with a function rather than being introduced manually more than once. In relation to this, at one point the GD noticed that the same figure had been copied to another cell with one decimal number missing and said:

"We cannot let this happen. We have to be very precise. We have to tune this as accurate as possible. If the figure in one cell appears as 0,512 , then it has to be copied as 0,512 , not as 0,51." - GD (meeting on 2011)

The objective of the meeting was to review the various worksheets of the new accounting system. Moreover, the system was clearly focused on costs as it was intended to calculate costs related to raw materials, transportation, labor, payroll, utilities, and maintenance, among others. At some point the GD said the following

"The idea is to be able to make charts of everything" - GD (M eeting on 2011)

Moreover, the only people really participating in the conversation were the GD, the GM 2, and the Financial M anager. On the other hand, the Plant Manager and the Public Relations Manager stayed quiet during the whole conversation. During a small break I asked the Plant Manager about the new tool: 
"It looks good [then laughed]. At least it seems more than what we had before [...] This information [however] is not very clear for me" - Plant Manager (interview on 2011)

At this point, it was clear that the idea of the GD of institutionalizing cost or financial consciousness on his board members was not yet a reality. In fact, the Financial Manager seemed to be more reactive towards the use of the management accounting tool, rather than being proactive. After the meeting, the GD made the following remark:

"I had to develop this tool by myself because if it needed to be done quickly because we need to understand our margins clearly [...] We have already realized that our exported cassava was too expensive for the price that we had set" - GD (interview on 2011)

The following personal interview with the GD took place on December 2011. The questions were clearly focused on assessing the development of the management accounting tool and the overall institutionalization of cost consciousness in the organization. The interview, however, was very short and could be summarized with the following quote from the GD:

"The system is going well. We are fine-tuning it and it is getting more accurate with time

[...] you should talk with [the General Manager 2], she is the person who know about this."

- GD (interview on December 2011)

Therefore, the GD suggested his involvement with the implemented control system had become more passive. Interestingly, when asking the GM 2 about this, she answered:

"The people who are now in charge of updating the system are [the Plant Manager] and [the Innovation assistant]." - GM 2 (phone interview January 2012)

Additional interviews were conducted by telephone and via emails during the first months of 2012. The following subsection provides a summary and analysis of my interventions in Kopla.

\section{Summary of interventions in Kopla}

The interviews suggest that the Kopla staff understood that the GD was 'business' oriented. In fact, some of them (e.g. the GM 1 and the Plant M anager) seemed to be eager for advice and for suggestions about how to achieve the goals set by the GD. However, given the poor financial performance of the company, the GD decided to substitute the GM1. Hence, this case shows financial performance distress as a driving force behind cost consciousness.

Furthermore, the GD blamed the lack of understanding of costs and "a bad sales-force" as the main reasons for the company's poor financial results. Moreover, he believed that the bad salesforce was partly due to the inability to correctly price the products of the company as a result the low cost related knowledge.

Hence, in early 2011 the GD introduced a MAS with the aim of "increasing cost consciousness" among the key members of his staff (e.g. the new GM, the Plant Manager, the Financial M anager, and the Innovation Assistant). The M AS was developed by the GD and some assistants during a short period of time. It is important to note that, although the GD had considerable experience in government tax and macroeconomic functions, he was not particularly knowledgeable about management or financial accounting; this, to his own admission.

The MAS is really an Excel file formed by multiple worksheets. In principle the MAS allows the calculation of various costs including product costs, raw material costs, labour costs, and 
maintenance costs. However, the system uses very simple cost allocations and requires all the data to be introduced manually. Moreover, in certain cases, the same data has to be introduced twice in different worksheets resulting in a clear risk. Therefore, one could reasonably argue that this M AS represents a very rustic and rudimentary system. However, as it supposed to serve the purposes of management accounting (i.e. identifying, measuring, collecting, analyzing, preparing, interpreting and communicating financial information used by management to plan, evaluate, and control within an organization) it can be considered as a MAS.

Moreover, the adoption of the MAS by the other members of the organization was not really an option. In Colombian society, the hierarchy structure can be considered to be very vertical. In other words, in many cases people do not question their superiors, especially not openly in public. In addition, the region where the country resides is considered by many Colombians as a particularly conservative area of the country; hence, reinforcing more the status and power of superiors. This could be perceived as a societal type of institution which is carried in the organizations. Furthermore, the GD had been in high positions in governmental functions which had made him a public figure to a certain extent. Hence, Kopla's staff was being directed not only by a boss, but by a recognized character at a national level.

The GD initially allocated the responsibility of the MAS to the GM and his involvement with the system became very passive. Moreover, although the GM did use the MAS, the Plant Manager and the Innovation assistant became the ones responsible for the task of updating the system. However, the Plant Manager, in particular, had been very outspoken, at the beginning of my initial visits to the company, about his lack of financial knowledge and limited understanding about cost management.

Finally, the Kopla case is interesting for various reasons. First, the organizational culture of the company before the GD's acquisition was very different to the principles which he intended to institutionalize in his company. Hence, this case reflects the efforts for conducting a type of organizational culture change or institutionalization of new basic assumptions and taken-forgranted ways of thinking. Second, the GD developed a MAS aiming at cost consciousness; hence, one could reasonably argue that the MAS was encoded with the GD's basic assumptions and institutionalized principles with respect to cost consciousness and maximizing profits. Therefore, this case illustrates very well many of the discussions in previous chapters where MASs take a central role in the processes of management accounting change. Third, although the implemented MAS is a very rudimentary system, the company did not use systems before the implementation which aimed at similar objectives. The only system which supported financial and accounting related functions was a database collecting the company's bookkeeping data for reporting revenue, overall expenses, and profit. One could reasonably assume that this situation was partly a result of the original founder's lack of interest for financial performance. Fourth, the company's staff was not very financially literate. Even the first financial manager of the company (after the acquisition by the GD) seemed to be relatively unknowledgeable about basic financial notions and did not have much experience as she had recently graduated from University studies. In addition, the other Board Members included people who had very limited financial and cost management literacy. In fact, the only person who had extensive experience in financial related issues was the GD. However, this experience was mainly related to macroeconomics and taxation. Fifth, my involvement allowed me to become much familiarized most of the key members of the staff (i.e. the board members). Therefore, I was able to perceive the relationships between status and power between the actors. Finally, sixth, this case describes the processes of management accounting change in a Colombian company. Hence, this study is 
original and provides insight not only on a Colombian organization's management accounting practices, but also on other issues such as Colombian culture and hierarchical relations.

Summarizing, this subchapter has reviewed an interesting case study where a new owner of a company tries to institutionalize cost consciousness in its organization through the implementation of a MAS. This case study provides a valuably opportunity to illustrate how the study of cost consciousness could be analyzed in practice following the discussions on previous chapters.

\subsection{Illustration of Formulation on Case Study}

This subchapter focuses on integrating the results of the case study into the formulation presented in the theoretical exercise in order to illustrate how the institutionalization of cost consciousness could be analyzed in practice.

Institutional Realm: List of Institutions

My visits to Kopla allowed me to familiarize myself with most of the staff that had been working for the original founder of the company. This became very important for trying to decipher the institutional principles which prevailed before the company was purchased by the GD. Both the GM 1 and one of the accountants were very open at explaining how 'good of a person' and 'caring' the original founder was, and, furthermore, how not-business oriented the company used to be. Hence, it is possible to assume that the institutions prevailing in the company were not financially and cost consciousness oriented, regardless of the interpretation of these notions.

Moreover, the new owner (i.e. the GD) clearly contrasted the original founder in many ways. Particularly, the GD was very emphatic about the need to treat the company as a business. Furthermore, the GD interpreted the poor financial performance of the company as a result of a lack of understanding of costs which lead to problems in pricing and competition. Hence, the GD developed a MAS to institutionalize the "consciousness of costs" in the board members of his organization. M oreover, the GD had a very holistic understanding of cost consciousness similar to the interpretations by various management accounting scholars (at least in terms of conceptual broadness). In other words, for the GD, cost consciousness was not only about cost reduction, but more about understanding costs and their relationship to activities in the company. Hence, the GD's view on cost consciousness supports the conceptual broadness related to the notion which the conceptualizing exercise brought forward.

The institutionalization of cost consciousness, however, was a particularly difficult task in Kopla given the level and differences with respect to the financial literacy between the board members. Particularly, the Plant Manager, who became one of the people responsible in updating the MAS, was very unfamiliar with very basic financial concepts such as profit margin. However, the language of the board members was indeed changed after some time. Terminology such as "costo kilo" (i.e. cost per kilo), "costo hombre" (i.e. referring to labour costs), and "costos por turno" (i.e. labour shifts costs) started to be used among the board members, including the Plant M anager. These notions appeared in some of the Excel columns of the MAS and, it is worth to note, I never heard these terms being articulated by the board members before the implementation of the MAS. Hence, one can reasonably argue that the MAS had introduced this vocabulary into the board members. Therefore, as language is part of the most visible level of 
organizational culture (i.e. artefacts), it is possible to argue that the cost consciousness encoded in the MAS - was now reflected in part of the most superficial culture among the board members of the company.

Moreover, all of the board members seemed to have become very aware of the need to be profitable and consider the costs involved in the activities of the company. In fact, given the hierarchical structure in the company, the board members seemed to believe that a better understanding of costs would lead to higher profits. Hence, one can reasonably argue that the interpretation of cost consciousness by the GD had become an espoused belief in the key members of the organization. In addition, espoused beliefs represent the intermediate level of organizational culture. Therefore, cost consciousness was reflected in the two most visible levels of Kopla's culture. Moreover, an very importantly, a better understanding of costs leading to higher profits can be considered as the central (intended) result of cost consciousness from the perspective of the case study.

Furthermore, espoused beliefs and values, if successfully repeated may become the basic assumptions at the core of an organizational culture. Unfortunately, it was not possible for me to assess if cost consciousness was part of the basic assumptions of Kopla's board members. Therefore, it is not possible for me to assess if cost consciousness was institutionalized in the company as a feature of the company's culture. However, Guerreiro et al. (2006, p. 225) mention that institutionalization occurs at different levels in different actors. In this sense, I could argue that cost consciousness was institutionalized to a certain level; but, it was not possible to assess if it represented the most invisible levels of the organizational culture. Nevertheless, although acknowledging this incapability with respect to being able to affirm that cost consciousness was institutionalized in Kopla, it was possible to sense that the institutionalization of cost consciousness was underway, and, furthermore, that various elements were interacting in this process - as the following subsections discuss. Using the vocabulary by Berger and Luckmann (1966, pp. 57-58), the institution of cost consciousness seemed to be in nucleo or in statu nascendi.

Modalities: Description of Management Accounting Systems

Before the implementation of the new MAS, the company used a database to collect the bookkeeping related information for reporting sales, overall expenses, and profit. This system, however, did not allow insight in product, raw material, or labour costs. Moreover, although it was possible to assess to a certain extend the profitability related to the different products, it was not easy for the key actors to retrieve this information in a fast and effective manner.

The MAS developed and implemented by the GD arguably represented a kind of innovation in the company. The system was divided into 24 worksheets, each worksheet with a specific purpose (e.g. calculating labour hours, raw material costs, maintenance costs). The objective of the MAS, according to the GD, was to increase the level of cost consciousness in the company by improving the capacity to calculate expenses related to products, raw materials, labour, administration, and maintenance.

However, the introduced MAS is a very rudimentary MAS allowing very simple cost allocations and, furthermore, its architecture was not very comprehensive. Nevertheless, despite its rustic nature, it was seen as an innovative and sophisticated tool when compared to the systems which the staff was accustomed to. It is difficult to assess the appropriateness of this system for this 
organization. On the one hand, a more sophisticated costing system could provide more security and better costing related results. However, this would involve the allocation of more financial resources, which, at the moment, were not available given the financial situation of the company.

Modalities: Rules

Although the MAS was not accompanied by a formalized set of rules such as a procedure manual, Quinn (2011, p. 349) states that Excel spreadsheets can be considered as a rule per se "since actors can refer to it for guidance." Therefore, the MAS represented not only a system, but also a set of rules.

Modalities: Routines

Although the control of costs was conducted in some level at the company before the implementation of the MAS, the MAS resulted in cost control to become a routine among some of the key members of the organization. The MAS required not only a constant update by the Plant Manager and the Innovation Assistant, but also revisal and presentation by the GM. Moreover, while the updating occurred at weekly basis, the revisal and presentation was conducted on a monthly basis. Hence, this routine is referred to as the routine of control.

Routines can be analyzed by analyzing their ostensive and performative aspects. The performative aspect of routines - observable through past action - "involves actions taken by specific people at a specific time, when they are engaged in an organisational routine" (van der Steen 2011, p. 513). Moreover, the performative aspect involves creating, modifying and maintaining the ostensive aspect of routines.

In the case of Kopla, the performative aspect of routine was evident in maintaining as the Plant $M$ anager and the Innovation assistant updated (i.e. maintained) the MAS on a weekly basis. This particular task was considered to be burdensome by the Plant M anager as he considered it to be somewhat misaligned from the more practical aspects of his work which he was more familiar with. However, with time, the Plant Manager became more familiarized with the MAS, and this allowed him to conduct the task in a faster and more effective way. Moreover, the GM had to analyze and present this information to the board on a monthly basis resulting in having to create Power Point presentations and reports were the most important results would be highlighted. Therefore, the performative aspect was also evident with respect to creating. In addition, the GD had stressed the need to "tune" the MAS so that the calculations would provide more accurate information. Hence, between the Plant Manager, Innovation Assistant, and GM, some of the figures are periodically modified according to their production results. Therefore, one can also say that the modifying purpose of the performative aspect of the routine of control was also evident.

Moreover, according to van der Steen (2011, p. 511), the ostensive aspect of routines "is encoded in particular expressions and languages, rules, standard operating procedures, and other artefacts". Moreover, the ostensive aspect of routines may have a significant tacit component, may be codified as a standard procedure, and may exist as a taken for granted form (Quinn 2011). The control routine in Kopla resulted in the production of reports and Power Point presentations mentioned above. 
Modalities: Loose Coupling

The MAS was implemented not only to build better capabilities for costing, but also to allow better assessment for the pricing of the products. However, according to the GM, the results of the MAS have not yet affected the pricing related activities in the company. Hence, one could reasonably argue that the MAS has been, to a certain extent, implemented in a ceremonial manner.

However, I would not argue that loose coupling occurred in Kopla "from the ongoing reproduction of routines" in the way that van der Steen (2011, p. 532) explains. Moreover, this case also seems to differ from the loose coupling which may result from the attempt to secure legitimacy from external forces (Siti-Nabiha and Scapens 2005).

The loose coupling in Kopla is better explained by Lukka (2007) who argued that the implementation of a new system may encounter resistance as the organizational status quo is disturbed. In a way, the basic assumptions of the GM seemed not to be completely aligned with the supposed purpose of the MAS because the system did not provide real pricing solutions for the company. Hence, although the GM could acknowledge certain information related to the costs of the products, her decisions concerning the price of the products were never really influenced by the information gathered from the MAS.

Realm of Action: List of Actors

The key actors involved in the process of institutionalizing cost consciousness in Kopla were the General Directors, the first General Manager, the second General Manager, the Plant Manager, the Innovation Assistant, and the Financial Manager. Their main roles in this process are described as follows:

- The General Director (GD). The GD acquired the company at the turn of the millennium from the family of the deceased original founder of the company. The GD had recently resigned from his position as a director of a very important intra-governmental function related to taxes and customs. The GD is currently around his 50s and has a degree in Law. His experience has been mainly in the public sector where he also held renowned positions in a Ministry in charge of macroeconomic affairs. In various ways, the GD resembles the Managing Director (MD) in the study by Burns (2000). Particularly, both characters were quite unsure about the capabilities in their staff. Moreover, while the MD was focused on institutionalizing results orientation, the GD was focused on institutionalizing cost consciousness.

- The first General Manager (i.e. GM1). The GM 1 who I met during my first interventions used to be the manager under the administration of the original founder of the company. She holds a University degree and had experience in managing positions for various years. However, since the first meetings she seemed to be unsure about the new administration of the company as it was very different to what she had been accustomed to during the previous years. The new business focus, she claimed, was good for the company, but needed time for one to get accustomed to. However, the financial situation of the company resulted in the decision of not continuing her contract. 
- The second General Manager (i.e. GM2). During my first visits, the GM 2 was in the position of Quality M anager. Although my interaction with her (while she was the Quality Manager) was limited, the GD mentioned in more than one occasion that the only staff member who met his expectations was her. Therefore, it was not a surprise when she was selected as the new General Manager (GM2) of the company. Furthermore, the GM2 had also a University degree in agronomy but also showed, according to the GD, an aptitude towards management. In fact, this aptitude was enforced as part of her tasks included producing accounting information. This observation supports Burns (2000) when he states that much of the accounting information in some companies is produced by managers rather than the accountants.

- The Plant Manager. During my first interventions, the present Plant Manager did not hold a management position as such and was considered to be some kind of a "chief of the plant" dealing directly with the labor and the mechanics who fixed the machines. When he was promoted to Plant Manager, however, his new job description still included most of his old responsibilities. The Plant Manager is the only Board Member without a University level degree as he holds a degree from a Technical School in agronomy (in Colombia, the difference between Universities and Technical Schools is vast with respect to people's perception. This perception is re-enforced by the salary gaps between those with University degrees and those with Technical degrees). From the beginning, the Plant Manager was very keen in letting me know about his lack of knowledge with respect to financial and management accounting. However, once he was promoted, and became part of the company's Board, he was faced with the need to acquire some financial related literacy. This literacy, however, seems to have been enforced through the updating of the MAS.

- Innovation Assistant. The Innovation Assistant is the board member with whom I developed the least amount of familiarity. She arrived after the implementation of the MAS and I did not have the opportunity to interview her personally. However, she became an important actor in this process as she shared, together with the Plant Manager, the task of updating the M As.

- Financial Manager. Curiously, the Financial Manager had no real interaction with the MAS. Her task was strictly oriented to the financial reporting of the company. M oreover, her involvement in the company initiated soon after my first intervention. Hence, although she did not play an important role in the implementation or use of the MAS, I became more familiarized with her when compared to the Innovation M anager.

- Other Actors. Although my formal interviews were held with the Board Members of the company, I did have the opportunity, in more than one occasion, to briefly talk with some of the accountants and the plant workers of the company. However, I consider their status and power low enough for them not to have any significant impact in the institutionalization process.

Finally, all of the actors involved in this process can be considered to be internal to the company. However, I believe that it might be important to also include myself in this list. Although I did not actively intervene in the process of institutionalization, I did interact between the actors, and, in certain cases, commented about my experience with the GD. However, as far as I am concern, 
my passive participation in the organization during the last years had no effects in the institutionalization process of cost consciousness.

\section{Realm of Action: Level of Status and Power}

I draw on the Table presented by Länsiluoto and Järvenpää (2010, p. 218) to summarize my assessment of the power and status of the actors during the implementation of the MAS (Table 13).

Table 13. Degree of Power and Status in Kopla Board members

\begin{tabular}{|l|l|l|}
\hline \multicolumn{1}{|c|}{ Actor } & \multicolumn{1}{c|}{ Power } & \multicolumn{1}{c|}{ Status } \\
\hline GD & High & High \\
\hline GM2 & Medium $\rightarrow$ High & High \\
\hline Plant M anager & Low $\rightarrow$ Medium & Low $\rightarrow$ M edium \\
\hline Innovation Assistant & Low & Low $\rightarrow$ M edium \\
\hline Financial Manager & Low & Low \\
\hline
\end{tabular}

As noted, Lovaglia et al. (2003, p. 109) define power as "the structurally determined potential for obtaining favored payoffs in relations where interests are opposed"; influence as what occurs "when a person's opinion or behavior changes to conform to the suggestion of another without the threat of punishment or the promise of reward"; and status as "a person's position in a group's prestige hierarchy". Defining influence is important, the authors argue, as an individual's status determines his/her influence.

The GD, as explained, has both high power and status in the company not only due to his ownership of the company, but also because he is a national recognized pseudo-political figure. However, the role of the GD in Kopla changed during the implementation and use of the MAS. This change is similar to the one observed by Länsiluoto and Järvenpää (2010, pp. 209, 210; 215; 220) where the CEOs of the organization which they studied initially tried to promote throughout their organization "a profit-oriented culture, particularly the aspect of being highly costconscious" and "emphasize profit orientation and to highlight the revenue side of profit consciousness". However, the authors note, the central and active role of the CEO at the beginning of the process transformed into a more passive one. Moreover, the authors note that the high degree of power of the CEO allowed a change in organizational culture, the status lessened during the implementation of the systems due to the passive role in the later stages. This, again, seems quite similar to the case of the GD in Kopla who could be reasonably considered as a powerful actor throughout the process (even in the later parts of the implementation he had the power to terminate or modify the system to his own will) and whose status, nevertheless, was reduced due to the distance that he took from the daily-operations and "tuning" of the management control system. However, one could also argue that it is not that the status lessens, but that the need to exert influence lessens through time. In this way, the degree of status remains relatively unchanged but will be noticeable only in a situation which requires it. In other words, the CEO and GD in the respective cases still have high status which, given the circumstances, are not reflected in the same manner as during the early stages of the implementation.

The GM2, moreover, had a high power during the implementation, but not as high as the GD. She (the GM2) was not involved in the development of the MAS, therefore her participation 
during the implementation of the system was relatively passive. However, once the system was introduced and it began to be used, her power increased, perhaps even higher when compared to the GD as the latter commented to me on one occasion that the GM 2 was "the one who was now in charge" of the use and "tuning" of the system. In terms of status, the GM 2 enjoyed high levels throughout the process, especially after the implementation once she became the person responsible for analyzing the information and answering questions about the data.

The Plant $M$ anager enjoyed neither high power nor status. The lack of University degree seemed to be an issue pointed by more than one of the other Board members. However, his power was not as low as the innovation manager's given that he had been in the company for many years and seemed to be respected by the labour and some of the other staff members.

The innovation manager, given her recent entry into the company had the lowest amount of power. Finally, the Financial Manager had both low power and status as she was not really involved with the implementation or use the MAS.

Realm of Action: Types of Power

With regards to power over resources, the GD has clear access to prestige, control of money, rewards and sanctions. Therefore, power over resources was evident throughout the intervention. As the major owner of the company, the GD inflicted a great amount of influence in the most important decisions made in the organization. Although the GM 2 had power in the daily activities, it could not be compared to the power of the GD. However, the GM 2 did have access to power over resources with respect to the other members of the organization as she was in charge of providing rewards and sanctions. M oreover, power over resources could be the main explanation behind the quick implementation of the MAS, even if it was misaligned with the prevailing basic assumptions in the company which were held before the new GD came in the organization. Hence, one could reasonably argue that power over resources accompanied the role of the GD during the early stages of the implementation; and, furthermore, it also supported the role of the GM 2 in later stages.

The case study in this dissertation provides insight into power of processes as the owner of the company works from 'behind the scenes' intervening directly at discrete moments but, simultaneously, exerting an overwhelming constant power. Within this respect, although the GM 2 seemed to be the most visible decision maker, she was not the most powerful one. One example supporting this assertion was that the MAS was fully developed by the GD without the consent or intervention of the GM2. Moreover, the MAS became fully implemented in the organization and the GM2 provided no visible challenge to the implementation of a system which challenged the prevailing ways of thinking in the company. However, the behind-thescenes power seemed to be reduced as the GD acknowledged that the MAS was being followed and that the company was being ran in the way in which he considered appropriate.

Moreover, Kopla provides a good environment for discussing power over meaning as the GD, who is seen by the employees as an expert and prestigious person, uses a new language in trying to persuade a new meaning inside the organization. The introduction of "consciousness about costs" and other terminology in the vocabulary of the staff, suggested an influence of the GD in the beliefs and values of the board members. In a way, power over meaning became very important for this case study as it was the use of a new language which provided a sense that cost consciousness was actually being institutionalized in the company. Therefore, one could 
reasonably argue that power over meaning may play an important role in the institutionalization of principles sharply challenging previously prevailing ones. This occurs given that this type of power is a mechanism resulting in changes in the behaviours of individuals which the individuals themselves might not be aware of; hence, do not necessarily trigger challenge.

The Kopla case allows the exploration of power of the system as the GD challenged the previously existing institutions inside the company. However, although it seemed that the members of the organization were opposing the institutional changes, the overwhelming power of resources of the GD overruled the power of the system. Moreover, the power over meaning, as explained above, resulted in subtle changes with respect to the language of the organizational members which seems to have reduced the power of the system.

Realm of Action: Resistance

Formal resistance to the MAS was not evident in this case as the power over resources of the GD did not allow any overt resistance to take place. Although the Plant Manager did show signs of feeling incapable of interpreting some of the financial terminology involved in the newly implemented MAS, he did not, as far as I am concerned, challenge the implementation or the use of the MAS. M oreover, although the GM 2 had not been consulted for the developing of the MAS, she abstained herself from exerting any kind of visible formal resistance. Once again, one could reasonably argue, a national and regional culture involving a steep hierarchical structure might be one of the main drivers explaining the lack of formal resistance in this case study.

However, some resistance associated with lack of capacity or knowledge was evident in the case, especially with respect to the Plant Manager who was not very familiar with financial related terminology or concepts. Financial related terminology seemed to be foreign to him and he expressed openly his lack of knowledge on the matter. However, the roles of the Plant Manager did not require him to deal with much of the new vocabulary; hence, little resistance of this kind was exerted by him. M oreover, the Plant Manager did begin using vocabulary which substituted terminologies which he previously used and was accustomed to.

M oreover, some degree of resistance with lack of capacity was also evident with respect to the Financial Manager and her skills in using Microsoft Excel. She, for example, seemed to have problems with respect to integrating information between the worksheets which required the need to insert some of the data in more than one location; therefore, resulting in clear risks related to consistency of information. However, this particular lack of capacity did not seem have much effect in the institutionalization process.

Finally, resistance as a result of mental allegiance was expected to occur as the M AS new ways of thinking which challenged the previous prevailing institutions in the company. However, due to the steep hierarchical structure, this resistance was also mitigated; hence, highlighting once again the importance of national culture in this case.

\section{Realm of Action: The Four M ovements}

The encoding of the institutional principles was clearly conducted by the GD who developed the MAS. The MAS was constructed to monitor and calculate product and overhead costs in the organization. Although, one could reasonably argue that the system was rudimentary and provides information which is not necessarily accurate, the MAS did reflect the GD's interest in 
heightening cost consciousness. Hence, this is a case where the MAS is encoded with institutional principles of a single actor (very powerful indeed) and not by the institutional principles prevailing in the organization. The encoding, moreover, was also reflected not only on the system per se but also on the discourses which the GD made before and during the implementation of the MAS. Hence, this movement provides space for criticizing the BSF as it does not differentiate between the different institutions in an organization, therefore, suggesting that the encoding of the institutional principles occurs between all the institutions of an organization and the MAS. Therefore, it is important to note that, at least in the case of Kopla, the encoding movement occurred between the institutions of one powerful actor and the MAS.

Moreover, the enactment and reproduction were mainly conducted by the Plant Manager, Innovation Assistant and GM2. The Plant Manager and Innovation Assistant focused on the modification and maintenance of the performative aspect of routines. As explained above, the performative aspect of the routines included the updating and maintaining of the MAS on a weekly basis. Most of this maintenance involved the introduction of data into the system related to, for example, raw material and labour costs. These activities were, however, already conducted prior to the implementation of the new MAS. Therefore, the encoding and reproduction of the performative aspects of the routine required few adaptations with respect to the encoding and reproduction of the performative aspects of the routines involved with the previous system. Therefore, the similarities between the activities before and after the implementation probably resulted in the marginal resistance which the actors of the organization exerted in the process.

Also with respect to enacting and reproducing the performative aspects of routines, the GM 2 created and presented the reports obtained from the MAS on a monthly basis. These reports, however, reflected activities which were very different with respect to the GM2's activities prior to the implementation of the MAS. However, little resistance to these movements was visible to me during my visits. Moreover, these activities, by the GM2, were the ones which seemed to be highly relevant to the institutionalization movement.

Finally the institutionalization process, as stated previously, is difficult to assess. On the one hand, the GM2, in her presentations, by referring to the results of the MAS, reinforces the need of the MAS. The Plant Manager, for example, acknowledging the periodical review of the MAS, stated his need to acquire the knowledge necessary for him to understand and perhaps even provide feedback about the MAS. M oreover, the other Board members also acknowledged not only the importance of these reports, but the importance of the MAS for the GD who, although exerting a more passive role in the company, still attended the majority of the Board meetings. Therefore, the GD still works 'behind-the-scenes', reinforcing continuously the need to maintain the MAS.

\section{Exogenous Forces}

In this case study, exogenous forces include competition and the overall production (and, therefore, price) of the raw food products in the country which are involved in the company's production. Moreover, the prices of these products are considered to be quite volatile, making the use of the rudimentary MAS for pricing not very useful. In fact, although the MAS was indeed maintained and updated in a periodical basis, the calculations of the costs of each product did not seem to have an effect in the pricing decisions. However, during the last Board meeting which I attended, the GD stated that the results obtained from the calculations of the MAS 
suggested that one particular product (i.e. the cassava in French-cuts) was being considerably overpriced. It should be noted that no decisions were taken at the moment concerning the pricing of this product.

Moreover, the purchase of the company by the new GD could be considered as a force which was initially driven from the exterior. In retrospect, this force was crucial for beginning the institutionalization of cost consciousness in Kopla. Furthermore, the financial distress of the company could also be considered as an important driver in the institutionalization process. However, this financial distress has both extra-organizational and intra-organizational components. While examples of the former include the competition and volatility of the raw material prices previously discussed, the latter include, at least according to the GD, the staff which was not competent to carry on the principles which he wanted to institutionalize.

\section{Change}

An institutionalization process can involve three dichotomies of change: formal versus informal; evolutionary versus revolutionary; and progressive versus regressive. With respect to the first dichotomy, the implementation of the MAS instituted new routines in the company; hence, one may reasonably argue that formal change occurred in the company. M oreover, informal change was also evident in the way in which some actors started to express themselves by using new language which was introduced to them together with the implementation of the MAS.

Moreover, with respect to the second dichotomy of change, the MAS resulted clearly in revolutionary change as it represented a new and, to a certain degree, innovative way of understanding and managing costs. Furthermore, the change was not revolutionary only with respect to the organization, but, according to the GM2, also with respect to the close competitors in the region. Within this respect, the use of new language could also be considered as an example of evolutionary change.

Finally, with respect to the third dichotomy of change, one could reasonably argue that the increase in financial literacy among the board members could be regarded as a kind of progressive change. This did not only allow better understanding by the board members about the costs incurred by the company's activities, but it also seemed to, at least in the case of the Plant Manager, to provide them with more pride about their own role and career development in the organization.

Therefore, it is important to take note of the importance which the introduction and use of new language has had in the process of institutionalizing cost consciousness in Kopla. This, actually, is in line with the importance of language in structuration and institutional theory as it was noted in various instances in previous chapters. Moreover, as noted, language is an important artifact in organizational culture which may allow deciphering the more invisible levels of the culture.

In sum, the formulation proposed in this dissertation has allowed the identification and analysis of various elements, mechanisms and forces relevant to the processes of management accounting change which occurred as a result of the implementation of a MAS in a small Colombian food processing company. Although at this point it is not possible to affirm that cost consciousness was institutionalized in the company, one could reasonably argue that, at least in some levels, the organizational culture in Kopla was changed into one which reflects cost consciousness as a growing feature. This means that the understanding of cost consciousness by the GD, which was encoded in the MAS, was reflected to a certain extent in the Board members 
of the organization. The understanding of costs, together with an assessment of their relationship to organizational activities and to the pricing of the products, became a more relevant topic in the day-to-day operations of the company. This relevance was reinforced during the monthly meetings where the GM 2 presented some of the results obtained from the calculations which the MAS provided. However, the results of cost consciousness which the GD expected (i.e. better pricing which lead to higher profits) have not been reached; therefore, one can reasonably argued that the institutionalization of cost consciousness has not been fully achieved. Nevertheless, analyzing the elements and mechanisms involved in the institutionalization process has allowed taking note of the importance of national culture, power over meaning, power over resources, and language in this particular case.

The following chapter will address the research questions of this dissertation by reviewing the discussions held in this chapter together with the results of the conceptualization exercise. This will allow synthesizing the theorization exercise. 


\section{Discussions: Contributions, Future Research, and Limitations}

The aim of this final chapter is threefold. First, this chapter discusses the central findings of this dissertation and their relationship with the contributions to the academic community. Second, the implications for future research will also be analyzed in this chapter as this work has built the foundations for future efforts aiming to further our understanding of cost consciousness. Finally, this chapter explores the main limitations of this study with respect to methodology and analysis of the results.

\subsection{Contributions and Implications for Future Research}

This dissertation provides two main contributions to the academic community:

1. Conducting a thorough literature review identifying the drivers and results associated to the notion from a management accounting perspective.

2. Conducting a theoretical exercise aiming to identify and analyze the organizational elements and mechanisms which may contribute to the institutionalization of cost consciousness from a management accounting perspective.

In the introduction of this dissertation, while the outline of the first contribution resulted in Research Question 1; the outline of the second contribution concluded in the formulation of Research Question 2 and Research Question 3. The objective of this subchapter is to discuss how these three research questions have been answered, therefore addressing, simultaneously, the contributions of this work in greater detail. In addition, given the exploratory nature of this study, the implications for future research will also be analyzed in this subchapter.

Before addressing the research questions, it is important to underline that, prior to this dissertation, only two management accounting articles - i.e. Shields and Young (1994) and Kurunmäki (1999) - focused on understanding the drivers of cost consciousness. However, it could be argued that the conceptualization of cost consciousness, per se, was not the focus of these two studies. Furthermore, although some authors have provided seemingly explicit definitions of cost consciousness, it is evident that most authors avoid defining the construct. Also, there is no evidence (to my knowledge) of previous scientific studies identifying or inferring the possible results of cost consciousness.

In sum, the following statement is worth underlying:

Prior to this work there was a lack of scientific literature addressing the conceptualization of cost consciousness and, moreover, there were only a handful of articles discussing possible drivers or providing definitions associated with the notion.

The previous statement highlights the importance of this dissertation for the scientific community and, furthermore, brings back into perspective the contributions of this work. It is important for the reader to take note that this dissertation was built on the review of scientific publications which had not been previously collected with the purpose of understanding cost consciousness. Hence, one could reasonably argue that not only is the topic of this dissertation 
novel, but that this work fulfills the primary objective of a PhD research program which is the creation of new knowledge (Lawlor and Donnelly 2010).

\section{On Research Question 1}

The first research question which this dissertation aimed to answer is:

What is cost consciousness as it is understood in the management accounting scientific literature?

Moreover, as explained in the introduction, this research question would be supported by the following sub-questions: what are the drivers, definitions, and results of cost consciousness? why is cost consciousness desired/undesired? And how can the cost consciousness notion be associated with organizational culture?

Before addressing these questions, I feel that it is important to acknowledge that the difficulties raised in the conceptual development of cost consciousness are, in fact, shared by most notions. O'Leary (1915), for example, argues about the existence of culture, regardless of the capability (or incapability) to define it in clear-cut terms. The author, moreover, highlights the limitations of the dictionary which were also raised throughout the discussions in the conceptual exercise. In addition, the author also argues that the use of a particular concept might change through time.

The first of the previously noted sub-questions was depicted early in this dissertation (Figure 4) highlighting the main elements which were intended to be identified and inferred from the literature. As the reader can recall, the literature review was meant to identify and infer the definitions (and interpretations), the determinants (or drivers), and the results associated with cost consciousness in management accounting literature.

From a linguistic composition point of view, cost consciousness can be considered as an endocentric compound where consciousness acts as a linguistic head, and cost acts as a linguistic modifier. While the linguistic head provides the notion with a broad conceptual realm, the modifier restricts and limits this realm in a way which may serve a field like management accounting.

Moreover, with respect to the interpretations of the notion, cost consciousness portrays an understanding of costs which may result in various organizational capabilities including an enhancement in evaluating alternatives in terms of financial outcomes and better monitoring of spending behaviour by managers.

In addition, cost consciousness has also been associated with organizational beliefs and organizational culture. However, this dissertation has emphatically expressed the need to differentiate between the notions of institution and organizational culture in order to avoid overlaps and conceptual discrepancies. Therefore, cost consciousness can potentially represent an institution acting as a feature of an organizational culture. Hence, this dissertation, also contributes to the extant literature on organizational culture and its relationship with institution, management accounting, and MASs. 
Furthermore, the discussions of this dissertation outline the following definition of cost consciousness:

Cost consciousness represents a feature of a business culture that allows the members of an organization to increase their understanding and knowledge of costs by enhancing organizational capabilities such as the monitoring of expenditures, budgeting, and the evaluation of alternatives in terms of financial outcomes.

It is important to note that this definition represents an understanding of cost consciousness from a management accounting perspective based on institutional and structuration theory. Clearly, if one would opt to understand cost consciousness by using other theoretical realms, the definition would change, perhaps dramatically.

Moreover, with respect to the drivers or determinants of cost consciousness, the literature review allowed me to identify and infer various determinants which management accounting scholars have associated with cost consciousness. Management Information Systems (MISs), MASs, outputs of MISs, Management Programmes, exogenous forces, and the role of actors are the main categories of these determinants. Also, the literature review allowed the identification of various results associated with cost consciousness. Although most of these reflect positive outcomes (e.g. better monitoring of spending behaviour), cost consciousness has also been associated (in a few cases) with negative results (e.g. myopic vision of the organization).

Therefore, this dissertation has not only defied the limitations of the dictionary with respect to cost consciousness, but has attempted to change the current use of cost consciousness (i.e. as a linguistic-apparel used to muddy up the scientific prose) into a more conceptually robust notion representing significant research opportunities - particularly in the management accounting field - by analyzing the linguistic composition of the construct and exploring how management accounting scholars have understood cost consciousness in the past.

Finally, I would like to raise five points with respect to implications for further research raised from the analysis of cost consciousness from a management accounting perspective. First, the literature of cost consciousness from a management accounting perspective could be supported by an analogous literature review on similar notions such as cost awareness. It would be very interesting to take stock of both the similarities and the differences with respect to the associations which management accounting scholars have made with these two notions.

Second, it was noted that cost consciousness has been used not only in management accounting literature, but also across various academic disciplines. Actually, it was noted that the notion appears commonly in health care and medicine related journals. Hence, the literature review on cost consciousness from a management accounting perspective might find support from an analogous review from other perspectives. The common use of the cost-consciousness construct in health care related journals might be read as a manifestation of the emergence of the New Public Management (NPM). Perhaps, whereas costs have been known to hold a central role in profit-seeking privately held companies, public sector organizations - such as hospitals and other healthcare centers - may have had, at times, less regulation with respect to cost management. Consequently, cost considerations in the public sector (e.g. public hospitals) might be become noteworthy and thus of interest also in scientific literature. Furthermore, striking conflicts are likely to arise with respect to the discussion of cost consciousness in the health care literature where the monetary value of life and its relationship with cost consciousness might raise interesting analyses. In this way, future research could draw from (and support) works which 
have dealt with NPM. For example, the work by Rautiainen and Järvenpää (2012) reflects on the normative ideas of the NPM trend in refining the typology of organizational responses suggesting how the choice of response depends on the institutionalized logic in the context of public sector accounting.

Third, given the importance of the linguistic composition of cost consciousness, future studies could focus on reviewing other notions and their respective conceptual development. These exercises could provide insight into how cost consciousness could be developed and, also, on its possible roles in academic and management accounting literature. Fourth, the notion of cost consciousness has been studied from English academic literature. In Spanish, moreover, the closest literal translation of cost consciousness would be translated into "consciousness about costs". Hence, the notion is not translated into a compound. Therefore, it might be interesting to study how the translation of the notion in different languages affects its interpretation. Fifth, market orientation has been raised as a benchmark for cost consciousness in terms of conceptual development. Hence, a deeper understanding of the path which market orientation has taken in this respect could also allow further insight into the possibilities related to cost consciousness in the management accounting field. It might be particularly interesting to take note of the particular publications which raised the attention of market orientation in an attempt to take stock of the steps which could be taken for furthering the interest for cost consciousness.

\section{On Research Question 2}

The second research question of this dissertation is:

Which organizational elements and mechanism can contribute to the institutionalization of cost consciousness?

This work has drawn on structuration theory, institutional theory and the Burns and Scapens Framework (BSF) to identify the intra-organizational elements (and some extra-organizational) and, moreover, examine the way in which these interact in a theoretical institutionalization of cost consciousness. Furthermore, although the use of these theories and the BSF in the management accounting field is nothing new, a thorough understanding of the elements of the BSF requires consideration of some very abstract and conceptually demanding notions. Routinization, for example, although explained by Burns and Scapens (2000), is carefully considered by Berger and Luckmann (1964) and Giddens (1979, 1984). In addition, reviewing Barley and Tolbert (1997) allows a better understanding of the structure of the BSF and allows one to easily trace the BSF to Giddens's modalities of structure.

Hence, this dissertation serves as a comprehensive quideline for any management accounting scholar who not only wishes to use the BSF, but also understand in a more comprehensive manner the mechanisms and elements which compose it.

Through this exploration, moreover, it was noted that there were various elements which interacted between each other in the institutionalization process. Management accounting systems (M ASs) take a central role in a process of institutionalization as they link the institutional realm and the realm of action allowing actors to interact between each other while drawing from the institutions and, simultaneously, reinforcing or modifying those institutions. Furthermore, some of the propositions and criticisms of the BSF were also identified, some of which became very important for the theorization exercise. Within this respect, the differentiation between the 
ostensive and the performative aspect, together with the inclusion of exogenous forces in the analysis, proved to be very helpful for the theorization on the institutionalization of cost consciousness.

Therefore, the organizational elements, mechanisms, and forces which interact in the institutionalization of cost consciousness include: the prevailing institutions in organizations, the organizational actors (where their influence depends on the degree of status and power which they have), organizational rules, the ostensive and performative aspects of routines, and the exogenous forces in the organization's environment.

These organizational elements are mainly drawn from OIE theories focusing on intraorganizational elements and processes of change. Some management accountants have favored the more traditional perspective as it allows them to examine, for example, how the implementation of a particular management accounting system affects the operations in an organization. However, future research could analyze cost consciousness from a new institutionalist view by taking a broader perspective and examine, for instance, how formal and informal institutional constraints have an effect in cost consciousness. Karhunen et al. (2008), who draw on new institutional theory, explain that while formal institutional constraints include laws and regulations; informal constraints include customs, norms and culture. Therefore, the overlap between old and new institutionalism is evident as the informal constraints include norms which, as explained, serve as modalities in the legitimation dimension of structure. In addition, this dissertation has also considered the role of organizational culture and, furthermore, the Kopla case indicated that national culture could be an important factor in the institutionalization process. Hence, new institutional theory could provide further insight for the understanding of cost consciousness. In a similar manner, the institutionalization of cost consciousness could also benefit from embracing more NIS ideas and approaches. The work by Rautiainen and Järvenpää (2012), previously mentioned, is one example from where one could focus on how the institutional responses (i.e. acquiescence, compromise, manipulation, avoidance, and defiance) may act in the process of institutionalizing cost consciousness.

However, the identification of the elements mentioned above was only a first step in theorizing on how cost consciousness could be institutionalized in an organization. In exploring further this process, Research Question 3 needs to be addressed.

\section{On Research Question 3}

The third research question which this dissertation aimed to answer was:

How do the organizational elements and mechanisms contributing to the institutionalization of cost consciousness interact between each other?

As explained, MASs serve as modalities allowing actors to draw from the institutions in their dayto-day activities, and, simultaneously, feed and modify the organization's institutional principles. Moreover, the linkages between the realm of institutions and the MASs, and, furthermore, between the MASs and the realm of action are built by four movements (i.e. encoding, enacting, reproduction, and institutionalization) where agents - and their interactions within the MASs play a crucial role. Therefore, in answering Research Question 3, I will focus on the four movements. 
On the Encoding of Institutional Principles

Barley and Tolbert (1997 p. 100) suggest that encoding can take place in various ways. The authors explain that encoding may take place during socialization through the internalization of rules and interpretations of behaviour appropriate for particular settings. Moreover, encoding can also occur through the reinforcement of existing systems. Furthermore, Burns and Scapens (2000) state that the process of encoding instantiates the existing meanings, values, and power which reside in the institutions of the organization.

However, as observed in the case of Kopla, the encoded institutional principles do not necessarily need to represent the institutions of the organization. A tough CEO (as mentioned in one in the interviews in Metso) can encode his own institutional principles in a MAS which is then implemented and enforced in the organization. This, clearly, highlights the importance of status and power in the institutionalization of cost consciousness.

Therefore, the encoding of a particular understanding of cost consciousness may occur between the MASs and the institutional principles of one actor which has enough power and status to challenge any resistance that may surge as a response of a challenge to prevailing organizational principles.

Hence, Figure 27 illustrates how the implementation of a MAS, to institutionalize cost consciousness, could be conceived by modifying the BSF with respect to the institutionalization of the principles of one powerful actor.

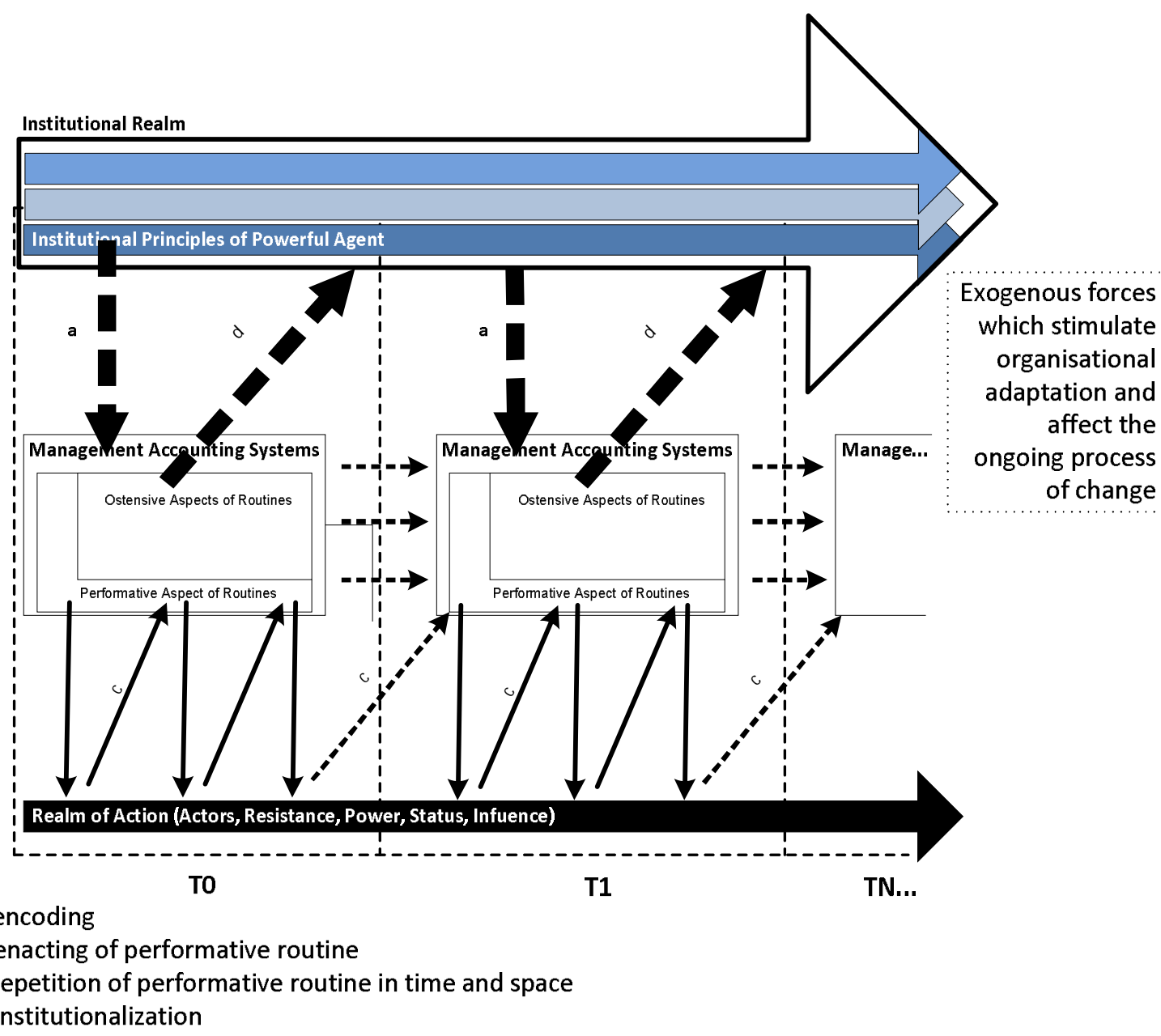

Figure 27. Institutionalization of cost consciousness by powerful agent implementing a M AS 
Moreover, although the literature warns that the process of institutionalization may fail if the encoded meanings and values challenge the prevailing institutions; the analysis of the case study suggests that, perhaps, in cultures with steep hierarchical structures, these types of challenges can be minimized. Heliste et al. (2007), drawing on new institutional theory, discuss about how the need for an authoritarian leadership and a clear hierarchy may affect institutional change. Therefore, once again, new institutional theory could provide support on understanding how informal institutional constraints may affect the institutionalization of cost consciousness.

Hence, the national or regional culture may have important repercussions in the institutionalization of cost consciousness in an organization. Cultures with very vertical hierarchical structures may represent fewer challenges for powerful and high status organizational actors.

Furthermore, noticing the influence of culture in the institutionalization process highlights the importance of exogenous forces. Actually, this dissertation identified various exogenous forces which may act as possible drivers of cost consciousness including market pressures, economic turndowns, and financial distress. Moreover, in the case of Kopla, financial distress, together with the GD's own financial ambitions, influenced the implementation of the MAS. However, one might ponder about the use of these types of pressures merely as excuses for the implementation or modification of a MAS. In this way, these pressures act not as drivers of cost consciousness but as vehicles for powerful agents to institutionalize a particular set of assumptions.

Management accounting literature has acknowledged the relationship between economic pressures and MASs (Hussein and Gunasekaran 2002). As explored, Granlund and Lukka (1998) identify four drivers of management accounting practices including economic pressures which underlie a considerable part of the global trend of management accounting practice; and, furthermore, include global economic fluctuations, increased competition, and advanced production and information technologies. Economic pressures, furthermore the authors argue, drive adaptive changes in management accounting practices through varying degrees of responsiveness. The authors also mention that global or economic recessions have increasing rapid global economic effects. In addition, Scapens (2006, p. 27) explains that as "organisations seek to be technically efficient, various economic pressures shape the nature of their management accounting practices; but they are also shaped by their attempts to conform to the expectations of their various stakeholders". Hence, these observations are in line with the results of this dissertation particularly with respect to the case study as the GD implemented the MAS partly as a response to the financial dire straits facing the company.

Therefore, economic pressures could be important elements in the driving of cost consciousness as they provide reasons to enforce an implementation or modification of a MAS which could shape the shared understanding of cost consciousness in an organization. Economic pressures could reinforce the power of an actor driving the required management accounting change, or they could also provide power to less powerful actors by allowing them to mobilize power over meaning to achieve their own goals.

Therefore, exogenous forces may act as drivers of cost consciousness by facilitating actors with reasons and excuses which could allow them to encode a MAS with their own institutional principles and avoid resistance. In this way, actors may be able to disseminate their own understanding of cost consciousness in the organization. 
One could also reasonably expect for economic pressures to drive cost consciousness in other ways. For example, high-level managers might, in a truly unselfish manner, react to economic pressures by implementing the necessary practices to institutionalize cost consciousness.

\section{On the Enacting and Reproduction of Performative Aspects of Routines}

The enacting and the reproduction of the performative aspect of routines are central activities with respect to the institutionalization of cost consciousness. As Burns and Scapens (2000) explain, while the enactment normally results from reflexive monitoring and application of tacit knowledge (practical consciousness) the reproduction involves the repeated behaviour of action. Within this respect, the literature review identified various uses of AISs which may act as drivers of cost consciousness including the allocation of costs, the comparison of revenues and budgets, the development of budgetary control, the establishment of budgetary goals, the participation of budget, and the identification of profit centers. Furthermore, as explained, these activities represent the performative aspect of routines as they might escape discursive explanations from the actors who conduct such activities.

If one is to uncover the nature of the relationship between these activities and the driving of cost consciousness it would be necessary to analyze each one in detail. I will now revise cost allocation as a driver of cost consciousness which should serve as an example for future research. Although intuitively cost allocation serves accounting purposes, Doost (1996) argues that cost allocation makes no difference in a company's bottom line: "If we have a pool of common and joint costs and allocate them through various means and formulas to our several products or services, the bottom line will probably not change." However, one could reasonably expect various authors not to agree with such a bold statement. Snyder and Davenport (1997), for example, explain that a fundamental problem in determining costs is to decide how to allocate these, particularly with respect to indirect or overhead costs. In addition, the authors argue, allocating costs is important for two reasons; namely, better economic decisions (including better decisions related to pricing) and a higher level of managerial motivation (related to the encouragement of lower-level managers to behave in ways that further the aims of the organization).

Interestingly, the GD in Kopla implemented the MAS to improve the pricing capabilities of the company and, furthermore, he encouraged some organizational members to behave in different ways in the company (e.g. paying more attention to costs and changing language and terminology).

Hence, cost allocation may act as a driver of cost consciousness if it is enacted and reproduced to the extent that it provides better pricing capabilities; and, furthermore, it encourages actors to change their behaviour in a manner which is aligned with the new institutional principles.

Furthermore, a brief exploration of developing and establishing budgetary control might also shed light on how the use of AISs may act as a performative aspect of routines serving as drivers of cost consciousness. Dunk (1993) states that budgetary control is effective in enhancing the performance of manufacturing activities. In addition, budgetary control (together with standard costing) has been considered to contribute towards greater efficiency with respect to labour productivity (Edwards et al. 2002). Moreover, Wahyudi (2009), in reviewing the relationship between accounting and budgeting, refers to the work by Arwidi and Samuelson (1993) to argue that accounting in the form of budgetary control displays the introducer and designer of the 
control as the real power holder in an organization, and, furthermore, strengthens a managerial culture. Hence, budgetary control serves as a vehicle of power; therefore, reflecting its domination dimension according to structuration theory.

In other words, the enacting and reproduction of activities which develop and establish budgetary control may drive cost consciousness by highlighting the power of the actor(s) who introduced and designed the control; therefore mobilizing the means necessary to change the behaviour of other organizational members.

Furthermore, as managers' performance is commonly evaluated on the achievement of budgetary objectives, managers practice tight budgetary controls which include defining, rewarding, monitoring and communicating organizational goals (Oak and Schmidgall 2009). Therefore, a budgetary control not only highlights the domination dimension of a MAS, but the signification and legitimation dimensions where the former provides meaning, and the latter allows actors to sanction behaviours. With respect to the provision of meaning, it was argued in the analysis of Kopla that power over meaning allowed the GD to change the behaviour of the Board members in very subtle ways. These changes were reflected, for example, in the use of new language.

Therefore, the enacting and reproduction of activities which develop and establish budgetary control may drive cost consciousness due to the budgetary controls' ability to provide a vehicle for power; and simultaneously, allow subtle changes in behaviour which evade resistance.

In addition, other uses of AISs related to budgetary actions were also identified as possible drivers of cost consciousness. Budget participation was found to have the largest effect on cost consciousness in the study by Shields and Young (1994). Shields and Young argued that budget participation is an effective and efficient means of facilitating the sharing of (and learning of) diverse and complex information by innovative workers. Furthermore, Shields and Shields (1993) suggest that budget participation influences organizational outcomes including job satisfaction, performance, and morale. In addition, Joshi et al. (2003) mention that budget participation allows the participant to feel more responsible for organizational goals.

With respect to budgetary participation, the Plant M anager in the Kopla case acknowledged the need to increase his financial knowledge in order to be able to understand the discussions being held during the Board meetings. Furthermore, being part of the Board was clearly satisfying for the Plant manager and raised his morale. Therefore, it seems that allowing the Plant M anager to participate in the Board meetings increased his job satisfaction, and for him to maintain that satisfaction (i.e. remain the Board) he increased his cost consciousness by increasing his cost management knowledge and changing his behaviour. In addition, cost management knowledge was also identified as a driver of cost consciousness by Shields and Young (1994).

Therefore, the enacting and reproduction of budgetary participation (by making actors feel responsible for the organizational goals) may drive cost consciousness as this kind of participation can influence changes in behaviour such as increasing cost management knowledge, which, in itself may be a driver of cost consciousness.

Within this respect, Anderson et al. (2010) examine a case which could be used as benchmark in the future for analyzing the association between budgetary participation and assessments of performance related to cost consciousness. Their study is valuable due to the criticisms with respect to how these associations have failed "to employ an objective, external measure of performance". 
In sum, the enacting and the reproduction of the performative aspect of routines hold a very important role in the institutionalization of cost consciousness. In addition once these routines are successfully reproduced over time, they become routinized to a point in which actors may struggle in providing discursive explanations of these activities. In other words, the actors draw on their practical consciousness to conduct these routines. In the case of Kopla, the Plant Manager, after reproducing his activities over time, changed his behaviour and language into one which reflected cost consciousness. Therefore, one could reasonably argue that it is the tacit and almost unconscious actions which ultimately reflect cost consciousness in an organization.

Therefore, cost consciousness is, in great part, driven by the practical consciousness which is inherent to the routinization and institutionalization processes in an organization. Hence, when being asked how to drive cost consciousness, people hesitate not necessarily because there is no such thing as cost consciousness, but because the actions which reflect cost consciousness are not necessarily part of their discursive consciousness.

\section{On the Process of Institutionalization}

Both Barley and Tolbert (1997) and Burns and Scapens (2000) describe the institutionalization movement as the entailing the objectification and externationalization of the patterned behaviours and interactions from their historical circumstances. Hence, opposed to the other movements, the relationship between the institutionalization movement and the activities of actors is vague and perhaps confusing. Crossan et al. (1999) actually argue that the process of institutionalization sets organizational learning apart from individual or group learning.

The analysis of the case study suggested that the institutionalization of cost consciousness may be pursued through presentations and the development of reports reflecting the ostensive aspect of routines. The GM2 in Kopla, while presenting the results of the MAS, underlined the basic beliefs and assumptions which the GD had expected would reflect a cost conscious mentality. In this way, she communicated to the other Board members the expectations which should be met in order to reflect the intended cost consciousness. Within this respect, the literature review identified reports which have also been associated as drivers of cost consciousness including monthly forecasting reports, and cost (variance) reports. In this way, there is a shift from MISs acting as the main drivers of cost consciousness, towards the ostensive aspects of routines being the drivers of the notion. I would like to note that it is not that the MISs are irrelevant but that the focus needs to be on the activities which these involve. Clearly, a costing budgeting system could reasonably be expected to perform better in the institutionalization of cost consciousness when compared to a human resource management system.

Hence, the institutionalization of cost consciousness may be conducted by presenting and developing forecasting and cost reports which reflect the ostensive aspect of routines and guide future behaviour of organizational actors.

Finally, with respect to organizational learning and its relationship with the institutionalization of cost consciousness, future studies could be approached by examining how literature on organizational learning may provide further insight on institutionalization processes. Kim (1998) recalls the definition of organizational learning as a process whereby knowledge is created, is distributed across the organization, is communicated among organizational members, has consensual validity, and is integrated into the strategy and management of the organization. In 
addition, institutional theory has been combined with organizational learning in the past (e.g. Newman 2000), therefore this option for future research could contribute to these discussions.

Therefore organizational learning literature may provide tools for analyzing how cost consciousness in the individual level may be transferred into the group, and ultimately on the organization.

In sum, this subchapter has synthesized the results of the conceptual and theoretical exercises in answering the three research questions; and, furthermore, has brought forward some possible future research options that may find important foundations in this work. However, as an exploratory conceptual and theoretical exercise which this dissertation is, it is subject to various limitations which should be considered.

\subsection{Limitations of the Study}

This dissertation is as an exploratory study conducted through a conceptual and a theoretical exercise aiming to provide the foundations necessary for furthering the understanding of cost consciousness. Hence, the limitations of this work arise from the capacity (or lack of capacity) of the methodological strategies in supporting these contributions.

Given that no prior work has focused on the conceptual development of cost consciousness, it was necessary to infer most of the interpretations, drivers, and results which management accounting scholars have associated to the cost consciousness notion. These inferences are problematic for a couple of reasons. First, it is quite probable that many authors did not allocate much thought in opting to use the cost consciousness or cost conscious concepts in their studies. Hence, the drivers, interpretations, and results could have been associated to similar concepts such as cost awareness. Furthermore, some of the inferences required a considerable amount of freedom as it was not always possible to be sure if, for example, a particular driver was really being associated to the notion or if it acted in parallel to the driving of cost consciousness. Therefore, although I tried to be as thorough as possible in identifying the relationships, it might be possible to criticize this approach for the considerable amount of subjective interpretation. However, to the defense of this approach, I believe that given the exploratory nature of this study, it was necessary to uncover as many possible hints from the literature, even if this resulted in some associations which would create debate.

Moreover, as this work, or extracts from it, has not been published (yet), the notion of cost consciousness remains fragile and awaiting acceptance by the academic community. Hence, I am fully aware of the possibility that scholars may be negligent to the introduction of a concept like cost consciousness into the field, regardless of the justifications presented in this study.

Furthermore, as noted, the literature review could have also considered other seemingly identical concepts such as cost awareness as it is a misconception to assume that a single searchphrase yields all that is available from the literature (Levy and Ellis 2006). Actually, the literature review identified one article (Jacobs 2005) where cost consciousness and cost awareness appear as clear inter-exchangeable notions. Therefore, another limitation of this study refers to the noninclusion of concepts as such in the identification of possible interpretations, drivers, and results.

In addition, this study has relied on institutional theory, structural theory, and on the Burns and Scapens Framework (BSF) for the theorization exercise. Therefore, the limitations of this study 
are intricately related to the limitations of these theories and framework in allowing a theorization on the processes of institutionalization and management accounting change. For example, it is not possible to take a very close look into the realm of action as the BSF provides a very holistic perspective. Furthermore, although exogenous forces were taken into account in the proposal, the way in which these may affect the institutionalization of cost consciousness was not thoroughly discussed. This, once again, raises the importance of bringing other theories, including other institutional branches (e.g. NIS), when theorizing on the institutionalization of cost consciousness.

Also, the theoretical exercise integrated the results of the literature review on cost consciousness with the proposed formulation. This integration is subject to two main limitations. First, the subjective interpretation in inferring the drivers, definitions and results of cost consciousness is clearly translated into the integration. Second, these associations required further subjective interpretation and some of the possible roles which, for example, the drivers of cost consciousness may serve, might not find consensus from other authors despite my best efforts to justify them.

As well, having used the case study as a tool only for illustrating the results of this dissertation's theoretical exercise, both the interviews and the observations drawn from it might be considered as underdeveloped or underemployed. Although, admittedly, I have been fully aware of these possible considerations, two main limitations inhibited a more comprehensive use of the observations obtained and inferred from the case study. First and foremost, the case study company is based in Colombia limiting my access and communication with the organization's employees. Telephone and email conversations were rarely fluent and personal visits proved to be by far the best channel to gather information. However, both the time required for visiting the company (due to the geographical distance) and the high travel costs did not allow me to gather the amount of data necessary for the further analysis of the case study.

One final limitation, very important indeed, has to deal with validity and reliability as discussed in the methodological chapter. As explained, validity techniques have been constructed on the basis of quantitative research. As this dissertation can be considered to be of a qualitative nature, those quantitative-grounded-validity techniques are inappropriate. Moreover, our knowledge on how to raise and ensure validity in qualitative research is still under debate. Particularly, with respect to management accounting interpretative research, there seems to be an emergence of a "crisis of validity" (Lukka and Modell 2009) which needs to be addressed. Within this respect, I should recall one of the strategies proposed by Lukka and M odell (2009, p. 14) which is to take firmly entrenched theories "as a rather general starting point and rely heavily on rich, emic accounts whilst leaving explanations somewhat open-ended and thus affirming the messy and often paradoxical nature of life in complex organisations". The illustrative case study did embrace a firmly entrenched theory (i.e. institutional theory and the BSF). In addition, I argue, the case study relied on emic accounts given my condition as a Colombian studying a Colombian company through intense visits. However, I do recognize the limitations with respect to rich amounts of data. As previously explained, geographical and time related obstacles did not allow me to visit the company in more occasions.

In sum, the exploratory nature of this dissertation results in various limitations. The limitations range from subjective interpretations to geographical distances. However, most of these limitations, I believe, represent those which can be expected whenever conducting an exploratory research as such. 
Nevertheless, I believe that this dissertation provides a firm ground for future research aiming to explore what cost consciousness is and, perhaps more importantly, how it may be driven in organizations. These new paths of research, I strongly believe, now hold foundations which are not based merely on hunches but on knowledge. 


\section{References}

Abernethy, M.A., and Vagnoni, E. (2004) Power, organization design and managerial behaviour. Accounting, Organizations and So ciety, Vol. 29 (3/4): 207-225.

Abrahamsson, G. and Gerdin, J. (2006) Exploiting institutional contradictions: The role of management accounting in continuous improvement implementation. Qualitative Research in Accounting \& M anagement, Vol. 3 (2): 126-144.

Agbejule, A., and Saarikoski, L. (2006) The effect of cost management knowledge on the relationship between budgetary participation and managerial performance. The British Accounting Review, Vol. 38: 427-440

Agbejule, A. (2011) Organizational culture and performance: the role of management accounting system. Journal of Applied Accounting Research, Vol. 12 (1): 74 - 89.

Ahmadjian, C.L. and Robinson, P. (2001) Safety in Numbers: Downsizing and the Deinstitutionalization of Permanent Employment in Japan. Administrative Science Quarterly, Vol. 46 (4): $622-654$

Anderson, S.W., and Dekker, H.C. (2009) Strategic Cost Management in Supply Chains, Part 2: Executional Cost Management. Accounting Horizons, Vol. 23 (3): 289-305

Anderson, S.W., Dekker, H.C., and Sedatole, K.L. (2010) An Empirical Examination of Goals and Performance-to-Goal Following the Introduction of an Incentive Bonus Plan with Participative Goal Setting. M anagement Science, Vol. 56 (1): 90-109

Andon, P., Baxter, J. and Chua, W.F. (2007) Accounting change as relational drifting: A field study of experiments with performance measurement. Management Accounting Research, Volume 18 (2): $273-308$

André R. (2008) Organization Behaviour - An introduction to your life in Organizations. Pearson Education, Inc., Pearson International Edition. New Jersey: 572 pages

Antonacopoulou, E.P. (2001) The Paradoxical Nature of the Relationship Between Training and Learning. Journal of M anagement Studies, Vol. 38 (3): 327-350

Ashforth, B.E. and Humphrey, R.H. (1993) Emotional labor in service roles: The influence of identity. Academy of Management Review, Vol. 18 (1): 88-115

Atkinson, R. (2004) Uses and Abuses of Cooperation in a Digital Age. Collection Management, Vol. 28 (1/2): 3-20.

Babbar, S. and Prasad, S. (1998) International purchasing, inventory management and logistics research. An assessment and agenda. International Journal of Physical Distribution \& Logistics Management, Vol. 28 (6): 403-433

Bacharach, S.B., Bamberger, P., and McKinney, V. (2000) Boundary Management Tactics and Logics of Action: The Case of Peer-Support Providers. Administrative Science Quarterly, Vol. 45 (4): 704-736 
Bagozzi, R.P. Yi, Y. and Phillips, L.W. (1991) Assessing Construct Validity in Organizational Research. Administrative Science Quarterly, Vol. 36 (3): 421-458

Bahmanziari, T., Odom, M.D., and Ugrin, J.C. (2009) An experimental evaluation of the effects of internal and external e-Assurance on initial trust formation in B2C e-commerce. International Journal of Accounting Information Systems, Vol. 10: 152-170

Baird, K. (2007) Adoption of activity management practices in public sector organizations. Accounting \& Finance, Vol. 47 (4): 551-569

Baird, K.M., Harrison, G.L. and Reeve, R.C. (2004) Adoption of activity management practices: a note on the extent of adoption and the influence of organizational and cultural factors. Management Accounting Research, Vol. 15 (4): 383-399

Barker, K. and Szpakowicz, S. (1998) Semi-Automatic Recognition of Noun Modifier Relationships. Proceeding. COLING '98 Proceedings of the $17^{\text {th }}$ international conference on Computational linguistics, Vol. 1: 96-102.

Barley, S.R. and Tolbert, P.S. (1997) Institutionalization and Structuration: Studying the links between action and institution. Organization Studies, Vol. 18 (1): 93-117.

Barringer, H.P. and Weber, D.P. (1996) Life Cycle Cost Tutorial. Paper presented at the Fifth International Conference on Process Plant Reliability, Houston, Texas, October 2-4.

Batt, R. (2002) Managing Customer Services: Human Resource Practices, Quit Rates, and Sales Growth. Academy of Management Journal, Vol. 45 (3): 587-597

Bauer, L. (2008) Exocentric compounds. Morphology, Vol. 18 (1): 51-74

Baxter, J. and Chua, W.F. (2003) Alternative management accounting research - whence and whither. Accounting, Organizations and Society, Vol. 28 (2/3): 97-126

Becker, M .C. (2004) Organizational Routines: A Review of the Literature. Industrial and Corporate Change, Vol. 13 (4): 643-677

Belcher, L.W. and Watson, H.J. (1993) Assessing the Value of Conoco's EIS. MIS Quarterly, Vol. 17 (3): 239-253

Belohlavek, R., Klir, G.J., Lewis III H.W., and Way, E.C. (2009) Concepts and fuzzy sets: Misunderstandings, misconceptions, and oversights. International Journal of Approximate Reasoning, Vol. 51 (1): 23-34

Bem, D.J. (1995) Writing a Review Article for Psychological Bulletin. Psychological Bulletin, Vol. 118 (2): 172-177.

Bennett, M., Bouma, J.J., and Ciccozzi, E. (2004) An institutional perspective on the transfer of accounting knowledge: a case study. Accounting Education, Vol. 13 (3): 329-346.

Berger, P.L. and Luckmann, T. (1966) The Social Construction of Reality. First Anchor Book Edition. New York, United States of America. 219 pages.

Bhimani, A. (2003) A study of the emergence of management accounting system ethos and its influence on perceived system success. Accounting, Organizations and Society, Vol. 28 (6): 523- 
Boland, R.J. (1996) Why Shared Meaning Have No Place in structuration theory: A Reply to Scapens and M acintosh. Accounting, Organizations and Society, Vol. 21 (7/8): 691-697

Boutell, W.S. (1964) Business-Oriented Computers: A Frame of Reference. The Accounting Review, Vol. 39 (2): 305-311

Bovier, P.A., M artin, D.P., and Perneger, T. (2005) Cost-consciousness among Swiss doctors: a cross-sectional survey. BM C Health Services Research, Vol. 5: 72-78

Broadbent, M., Weill, P., and St. Clair, D. (1999) The Implications of Information Technology Infrastructure for Business Process Redesign. MIS Quarterly, Vol. 23 (2): 159-182

Brown, A. (1998) Organizational Culture. Second Edition. Pearson Education Limited. 317 pages.

Bruining, $H_{.}$, Bonnet, M., and Wright, M. (2004) Management control systems and strategy change in buyouts. Management Accounting Research, Vol. 15 (2): 155-177

Budding G.T. (2004) Accountability, environmental uncertainty and government performance: evidence from Dutch municipalities. Management Accounting Research, Vol. 15 (3): 285-304

Burchell, S. Clubb, C. Hopwood, A. and Hughes, J. (1980) The Roles of Accounting in Organizations and Society. Accounting, Organizations and Society, Vol. 5 (1): 5-27

Burns, J. (2000) The dynamics of accounting change: Inter-play between new practices, routines, institutions, power and politics. Accounting, Auditing and Accountability Journal, Vol. 13. (5): 566-596

Burns, J. and Scapens, R.W. (2000) Conceptualizing management accounting change: An institutional framework. Management Accounting Research, Vol. 11 (1): 3-25

Burns, J. and Vaivio, J. (2001) Management Accounting Change. Management Accounting Research, Vol. 12 (4): 389-402

Busco, C. (2009) Giddens's structuration theory and its Implications for Management Accounting Research. Journal of Management and Governance, Vol. 13 (3): 249-260

Busco, C., Quattrone, P., and Riccaboni, A. (2007) Management Accounting Issues in Interpreting its Nature and Change. Management Accounting Research, Vol. 18 (2): 125-149

Busco, C., Riccaboni, A., and Scapens, R.W. (2006) Trust for Accounting and Accounting for Trust. Management Accounting Research, Vol. 17 (1): 11-41

Busco, C. and Scapens, R.W. (2011) M anagement accounting systems and organisational culture: Interpreting their linkages and processes of change. Qualitative Research in Accounting \& Management, Vol. 8 (4): 320-357.

Böer, G.B. (2000) M anagement Accounting Education: Yesterday, Today, and Tomorrow. Issues in Accounting Education, Vol. 15 (2): 313-334

Calder, B.J. Phillips, L.W. and Tybout, A.M. (1982) The concept of external validity. Journal of Consumer Research, Vol. 9. (3): 240-244 
Campanale, C., Cinquini, L., and Tenucci, A. (2010) Do Management Accounting Systems Influence Organizational Change or Vice-Versa? Evidence from a Case of Constructive Research in the Healthcare Sector. Available at SSRN: http://ssrn.com/abstract $=1656943$ or http:// dx.doi.org/10.2139/ssrn.1656943

Campbell, D.T. and Stanley, J.C. (1969) Chapter 5: Experimental and Quasi-Experimental Designs for Research. Experimental and Quasi-Experimental Designs for Research. Houghton Mifflin Company. Boston, United States of America.

Cangelosi, V.E. (1965) The Carnegie Tech Management Game: A Learning Experience in Production Management. Academy of M anagement Journal, Vol. 8 (2): 133-138

Caplan, E.H. (1968) Behavioral Assumptions of Management Accounting - Report of a Field Study. The Accounting Review, Vol. 43 (2): 342-362

Carmines, E.G. and Woods, J.A. (2005) Validity assessment. Encyclopedia of Social Measurement: 933-937

Carmines, E.G. and Zeller, R.A. (1979) Reliability and Validity Assessment, Sage Publications, Inc., United States of America. 72 pages

Carmona S., Ezzamel, M., and Gutiérrez, F. (1997) Control and cost accounting practices in the Spanish Royal Tobacco Factory. Accounting, Organizations and Society, Vol. (5): 411-446

Carruthers, B.G. (1995) Accounting, Ambiguity, and the New Institutionalism. Accounting, Organizations and Society, Vol. 20 (4): 313-328

Caston, V. (2002) Aristotle on Consciousness. Mind, New Series, Vol. 111 (444): 751-815

Cerulo, K.A. (2000) Overcoming rigid conceptualization: Culture, cognition, and new approaches to traditional ideas. Poetics, Vol. 28 (2): 1-4

Chan, J.W.K., Burns, N.D., and Yung, K.L. (2000) Environment-strategy fit: a study of Hong Kong manufacturing logistics. Logistics Information Management, Vol. 13 (5): 286-300

Chang, S.J. (1995) International Expansion Strategy of Japanese Firms: Capability Building Through Sequential Entry. Academy of M anagement Journal, Vol. 38 (2): 383-407

Chenhall, R.H. (2003) Management control systems design within its organizational context: findings from contingency-based research and directions for the future. Accounting, Organizations and Society, Vol. 28: 127-168

Chenhall, R.H. (2008) Accounting for the horizontal organization: A review essay. Accounting, Organizations and Society, Vol. 33 (4/5): 517-550

Chenhall, R.H. and Euske, K.J. (2007) The role of management control systems in planned organizational change: An analysis of two organizations. Accounting, Organizations and Society, Vol. 32 (7/8): 601-637

Chenhall, R.H., Hall, M., and Smith D. (2010) Social capital and management control systems: A study of a non-government organization. Accounting, Organizations and Society, Vol. 35 (8): 737756 
Chenhall, R.H. and Langfield-Smith, K. (1998a) The relationship between strategic priorities, management techniques and management accounting: An empirical investigation using systems approach. Accounting, Organizations and Society, Vol. 23 (3): 243-264

Chenhall, R.H and Langfield-Smith, K. (1998b) Adoption and benefits of management accounting practices: an Australian Study. Management Accounting Research, Vol. 9 (1): 1-19

Choon, K., and Wisner, J.D. (2003) A study of operations management constructs and their relationships. International Journal of Operations \& Production Management, Vol. 23 (11): 13001325

Chua, W.F. (1995) Experts, networks and inscriptions in the fabrication of accounting images: A story of the representation of three public hospitals. Accounting, Organizations and Society, Vol. 20 (2/3): 111-145

Chua, W.F. and Mahama, H. (2007) The Effect of Network Ties on Accounting Controls in a Supply Alliance: Field Study Evidence. Contemporary Accounting Research, Vol. 24 (1): 47-86

Clackson, J. (2002) Composition in Indo-European Languages. Transactions of the Philological Society, Vol. 100 (2): 163-167

Clarke, B., and Mia, L. (1993) JIT M anufacturing Systems: Use and Application in Australia, Vol. 13 (7): 69-82

Clemons, E.K. and Hann, I-H (1999) Rosenbluth International: Strategic Transformation of a Successful Enterprise. Journal of M anagement Information Systems, Vol. 16 (2): 9-27

Clemons, E.K. and Row, M.C. (1991) Information Technology at Rosenbluth Travel: Competitive Advantage in a Rapidly Growing Global Service Company. Journal of Management Information Systems, Vol. 8 (2): 53-79

Coad A.F. and Herbert, I.P. (2009) Back to the future: New potential for structuration theory in management accounting research? M anagement Accounting Research, Vol. 20 (3): 177-192

Coase, R. (1998) The New Institutional Economics. The American Economic Review. Papers and Proceedings of the Hundred and Tenth Annual Meeting of the American Economic Association, Vol. 88 (2): $72-74$

Collier, P.M. (2001) The power of accounting: a field study of local financial management in a police force. Management Accounting Research, Vol. 12 (4): 465-486.

Collier, P.M. (2005) Entrepreneurial control and the construction of a relevant accounting. Management Accounting Research, Vol. 16 (3): 321-339

Conrad, L. (2005) A structuration analysis of accounting systems and systems of accountability in the privatized gas industry. Critical Perspectives on Accounting, Vol. 16: 1-26

Coyte, R., Emsley, D., and Boyd, D. (2010) Examining Management Accounting Change as Rules and Routines: The Effect of Rule Precision. Australian Accounting Review, Vol. 20 (2): 96-109.

Crossan, M.M., Lane, H.W., and White, R.E. (1999) An Organizational Learning Framework: From Intuition to Institution. Academy of M anagement Review, Vol. 24 (3): 522-537 
Cruz, I., Major, I. and Scapens, R.W. (2009) Institutionalization and practice variation in the management control of a global/local setting. Accounting, Auditing \& Accountability Journal, Vol. 22 (1): 91-117

Davila, A. and Wouters, M. (2004) Designing Cost-Competitive Technology Products through Cost Management. Accounting Horizons, Vol. 18 (1): 13-26

Davis, G.F., Diekmann, K.A., and Tinsley, C.H. (1994) The Decline and Fall of the Conglomerate Firm in the 1980s: The Deinstitutionalization of an Organizational Form. American Sociological Review, Vol. 59 (4): 547-570

Davis, L.E. and North, D.C. (1971) Institutional Change and American Economic Growth. Cambridge, Cambridge University Press. United Kingdom. 292 pages

De Loo, I. Verstegen, B. and Swagerman, D. (2011) Understanding the Roles of Management Accountants. European Business Review, Vol. 23 (3): 287-313

Dedehayir, O. (2010) M easuring Reverse Salience in Technological Systems. Tampere University of Technology, Tampere.

Dekker, H.C. (2004) Control of inter-organizational relationships: evidence on appropriation concerns and coordination requirements. Accounting, Organizations and Society, Vol. 29 (1): 2749

Dekker, H. and Smidt, P. (2003) A survey of the adoption and use of target costing in Dutch firms. International Journal of Production Economics, Vol. 84: 293-305

Dennett, D. (2001) Are we explaining consciousness yet? Cognition, Vol. 79: 221-237.

Dent, J.F. (1991) Accounting and organizational cultures: A field study of the emergence of a new organizational reality. Accounting, Organizations and Society, Vol. 16 (8):705-732.

DiMaggio, P.J., and Powell, W.W. (1991) The New Institutionalism in Organizational Analysis. Chicago: University of Chicago Press. United States of America. 478 pages

Dimick, D.E. and M urray, V.V. (1978) Correlates of Substantive Policy Decisions in Organizations: The Case of Human Resource Management. Academy of Management Journal, Vol. 21 (4): 611623

Doolin, B. (2004) Power and resistance in the implementation of a medical management information system. Information Systems J ournal, Vol. 14 (4): 343-362

Doost, R.K. (1996) Cost allocation: what purpose does it serve? Managerial Auditing Journal, Vol. 11 (8): $14-22$

Dortmans, P.J., Curtis, N.J., and Tri, N. (2006) An Analytical Approach for Constructing and M easuring Concepts. The Journal of the Operational Research Society, Vol. 57 ( 8): 885-891

Douglas, M . (1986) How Institutions Think. Syracuse University Press. Syracuse, New York, United States of America. 146 pages

Douglas, D.M. (2003) Piecing Together Service Quality: A Framework for Robust Service. Production and Operations M anagement, Vol. 12 (2): 246-265 
Dunk, A.S. (1993) Reliance on Budgetary Control, Environmental Uncertainty, and the Performance of M anufacturing and M arketing Units. Asian Review of Accounting, Vol. 3 (2): 3-15

D'Aveni, R.A. and Ilinitch, A.Y. (1992) Complex Patterns of Vertical integration in the Forest Products Industry: Systematic and Bankruptcy Risks. Academy of Management Journal, Vol. 35 (3): $596-625$

Edwards, J.R, Boyns, T., and M atthews, M. (2002) Standard costing and budgetary control in the British iron and steel industry: A study of accounting change. Accounting, Auditing \& Accountability J ournal, Vol. 5 (1): 12-45

Eisenhardt, K.M . (1989) Building theories from case study research. Academy of Management. The Academy of Management Review, Vol. 14 (4): 532-550

Eldenburg, L. (1994) The use of information in total cost management. The Accounting Review, Vol. 69 (1): 96-121.

Emblemsvåg, J. (2003) Life-cycle costing. John Wiley and Sons Inc. 320 pages

Englund, G. and Gerdin, J. (2008) Structuration theory and mediating concepts: Pitfalls and implications for management accounting research. Critical Perspectives on Accounting, Vol. 19: $1122-1134$

Englund, H., Gerdin, J., and Burns, J. (2011) 25 Years of Giddens in accounting research: Achievements, limitations and the future. Accounting, Organizations and Society, Vol. 36 (8): 494-513

Ezzamel, M . (1994) Organizational Change and Accounting: Understanding the Budgeting System in its Organizational Context. Organization Studies, Vol. 15 (2): 213-240

Falkenberg, L. and Herremans, I. (1995) Ethical Behaviours in Organizations: Directed by the Formal or Informal Systems? Journal of Business Ethics, Vol. 14 (2): 133-143

Feldman, M.S. and Pentland, B.T. (2003) Reconceptualizing Organizational Routines as a Source of Flexibility and Change. Administrative Science Quarterly, Vol. 48 (1): 94-118

Feng, G. and Baruch, L. (2004) The Information Content of Royalty Income. Accounting Horizons, Vol. 18 (1): 1-12

Finlay, W. (1987) Industrial Relations and Firm Behavior: Informal Labor Practices in the West Coast Longshore Industry. Administrative Science Quarterly, Vol. 32 (1): 49-67

Fisher, L. (1970) The Politics of Impounded Funds. Administrative Science Quarterly, Vol. 15 (3): 361-377

Fisher, J. (1995) Contingency-Based Research on Management Control Systems: Categorization by Level of Complexity. Journal of Accounting Literature, Vol. 14: 24-53

Fisher J.G. (1998) Contingency Theory, Management Control Systems and Firm Outcomes: Past Results and Future Directions. Behavioral Research in Accounting, Vol. 10. Supplement: 47-64

Flanagan, O.J. (1992) Consciousness Reconsidered. MIT Press. Third Printing. United States of America. 256 pages 
Fleischman, R.K., Hoskin, K.W., and Macve, R.H. (1995) The Boulton \& Watt Case: The Crux of Alternative Approaches to Accounting History? Accounting and Business Research, Vol. 25 (99): 162-176

Fleischman, R.K., Boyns, T., and Tyson, T.N. (2008) The Search for Standard Costing in the United States and Britain. ABACUS, Vol. 44 (4): 341-376

Forster, G.W. (1932) The Effects of the Present Credit System on Southern Agriculture. Social Forces, Vol. 10 (3): 426-435

Foster, G. and Gupta, M. (1994) Marketing, cost management and management accounting. Journal of M anagement Accounting Research, Vol. 6: 43-77

Forza, C. (2002) Survey Research in Operations Management: a process-based perspective. International Journal of Operations \& Production Management, Vol. 22 (2): 152-194

Fridson, M. and Alvarez, F. (2002) Financial Statement Analysis: A Practitioner's Guide. John Wiley \& Sons. Third Edition. United States of America. 424 pages.

Gates, S., and Langevin, P. (2010) Human capital measures, strategy, and performance: HR managers' perceptions. Accounting, Auditing \& Accountability Journal, Vol. 23 (1): 111-132

Geertz, C. (1993) The Interpretation of Cultures. Fontana Press. London. 469 pages

Geiger, D.R. and Ittner, C.D. (1996) The influence of funding source and legislative requirements on government cost accounting practices. Accounting, Organizations and Society, Vol. 21 (6): 549-567

Gerring, J. (2004) What is a Case Study and What is it Good for? The American Political Science Review, Vol. 98 (2): 341-354

Gibson, B.C. and Earley, P.C. (2007) Collective Cognition in Action: Accumulation, Interaction, Examination, and Accommodation in the Development and Operation of Group Efficacy Beliefs in the W orkplace. Academy of M anagement Review, Vol. 32 (2): 438-458.

Giddens, A. (1979) Central Problems of Social Theory: Action, Structure and Contradiction in Social Analysis. London and Basingstoke. The Macmillan Press Ltd. 294 pages

Giddens, A. (1984) The Constitution of Society. Outline of the Theory of Structuration. Polity Press. Cambridge, United Kingdom. 402 pages.

Goffman, E. (1983) The interaction order. American Sociological Review, Vol. 48: 1-17.

Golafshani, N. (2003) Understanding Reliability and validity in Qualitative Research. The Qualitative Report, Vol. 8 (4): 597-607

Goodhue, D.L., Quillard, J.A., and Rockart, J.F. (1988) Managing the Data Resource: A Contingency Perspective, Vol. 12 (3): 373-392

Goodin, R.E. (1998) The Theory of Institutional Design. Cambridge University Press. United Kingdom. 228 pages

Grandey, A.A. (2000) Emotion Regulation in the Workplace: A New Way to Conceptualize Emotional Labor. Journal of Occupational Health Psychology, Vol. 5 (1): 95-110 
Granlund, M. (2001) Towards explaining stability in and around management accounting systems. Management Accounting Research, Vol. 12 (2): 141-166

Granlund, M. (2003) Management accounting system integration in corporate mergers: A case study. Accounting, Auditing \& Accountability Journal, Vol. 16 (2): 208-243.

Granlund, M. and Lukka, K. (1998) It's a Small world of Management Accounting Practices. Journal of M anagement Accounting Research, Vol. 10: 153-179

Greenberg, J. (2010) Managing Behavior in Organizations. Pearson Education Inc., publishing as Prentice Hall, New Jersey. Fifth Edition. 476 pages.

Greene, H.L. Goldberg, R-J., Beattie H., Russo A.R., Ellison R.C., and Dalen J.E. (1989) Physician Attitudes toward Cost Containment: The missing piece of the puzzle. Archives of Internal Medicine, Vol. 149 (9): 1966-1968

Greve, H.R. (1995) Jumping Ship: The Diffusion of Strategy Abandonment. Administrative Science Quarterly, Vol. 40 (3): 444-473

Groot, T. and Budding, T. (2004) The Influence of New Public Management Practices on Product Costing and Service Pricing Decisions in Dutch Municipalities. Financial Accountability \& Management, Vol. 20 (4): 421-443

Gross, R., Nirel, N., Boussidan, S., Zmora, I., Elhayany, A., and Regev, S. (1996) The influence of budget-holding on cost containment and work procedures in primary care clinics. Social Science \& Medicine, Vol. 43 (2): 173-186

Guerreiro, R., Pereira, C.A., and Frezatti, F. (2006) Evaluating management accounting change according to the institutional theory approach. Journal of Accounting and Organizational Change, Vol. 2 (3): 196-228.

Gummesson, E. (1993) Case Study Research in Management. M ethods for Generating Qualitative Data. Part 1. Preliminary script, Second revised version. Sweden: 3-63

Hafner, G.F. (1964) Auditing E.D.P. The Accounting Review, Vol. 39 (4): 979-982

Hall, R. (1993) A Framework Linking Intangible Resources and Capabilities to Sustainable Competitive Advantage. Strategic Management Journal, Vol. 14 (8): 607-618

Hamilton, W. (1932) "Institution", in Seligman, E. and Johnson, A. (Eds), Encyclopedia of Social Science, Vol. 73 (4): 560-595

Hardy, C. (1996) Understanding Power: Bringing about Strategic Change. British Journal of Management. Vol. 7, Special Issue: S3-S16

Harris, C.L., Ogbonna, E. (2001) Strategic human resource management, market orientation, and organizational performance. Journal of Business Research, Vol. 51 (2): 157-166

Hassan, M.K. (2005) Management accounting and organisational change: an institutional perspective. Journal of Accounting \& Organizational Change, Vol. 1 (2): 125-140.

Heliste, P., Karhunen, P., and Kosonen, R. (2007) Foreign Investors as Agents of Organizational Change in Transition Economies: Human Resource Management Practices of Finnish Firms in Estonia. EBS Review, Vol. 22 (1): 16-28 
Henri, J-F. (2006) Organizational culture and performance measurement systems. Accounting, Organizations and Society, Vol. 31: 77-103

Hermann, M. and Heller, J. (1997) Andean Roots and Tuber Crops / Andean roots and tubers: Ahipa, arracacha, maca, yacon. Institute of Plant Genetics and Crop Plant Research, Gatersleben/International Plant Genetic Resources Institute (IPGRI): 75-172. Obtained on the 13.04.2011 in: http:// www.cipotato.org/artc/artc_hermann/Arracacha.pdf

Hochschild, A.R. (1983) The managed heart: Commercialization of human feeling. Berkely: University of California Press. United States of America. 318 pages

Hodgson, G.M . (1998) The Approach of Institutional Economics. Journal of Economic Literature, Vol. 36, No. 1: 166-192,

Hofstede, G.H. (1998) Identifying Organizational Subcultures: An Empirical Approach. Journal of Management Studies, Vol. 35, No. 1: 1-12.

Holden, T. and Wilhelmij, P. (1995-1996) Improved Decision M aking through Better Integration of Human Resource and Business Process Factors in a Hospital Situation. Journal of Management Information Systems, Vol. 12 (3): 21-41

Hoskin, K. and Macve, R. (1994) Reappraising the Genesis of M anagerialism: A Re-examination of the Role of Accounting at the Springfield Armory, 1815-1845. Accounting, Auditing \& Accountability Journal, Vol. 7 (2): 4-29.

Huntington, S.P. (1968) Political Order in Changing Societies. Yale University Press. United States of America. 488 pages

Hussain, M.M., and Gunasekaran, A. (2002) Non-financial management accounting measures in Finnish financial institutions. Accounting and Finance, Vol. 14 (3): 210-229

Hussain, M.M., Gunasekaran, A., and Laitinen, E.K. (1998) Management accounting systems in Finnish service firms. Technovation, Vol. 18 (1): 57-67

Hyvönen, T. (2000) Toiminnanohjausjärjestelmät ja kustannuslaskenta, Tutkimus suomalaisten teollisuusyrityksen tietojärjestelmistä. Tampereen Yliopisto, Yrityksen taloustieteen ja yksityisoikeuden laitos.

Hyvönen, T., and Järvinen, J. (2006) Contract-Based Budgeting in Health Care: A Study of the Institutional Processes of Accounting Change. European Accounting Review, Vol. 15 (1): 3-36

Imhoff, E.A. (2003) Accounting Quality, Auditing, and Corporate Governance. Accounting Horizons, Vol. 17 (Supplement): 117-128

Jack, L. (2005) Stocks of knowledge, simplification and unintended consequences: the persistence of post-war accounting practices in UK agriculture. Management Accounting Research, Vol. 16 (1): 59-79.

Jackson, D. W. and Ostrom, L.L. (1980). Life Cycle Costing in Industrial Purchasing. Journal of Purchasing and Materials M anagement, Vol. 16 (4): 8-12.

Jacobs, K. (2005) Hybridisation or Polarisation: Doctors and Accounting in the UK, Germany and Italy. Financial Accountability \& Management, Vol. 21 (2): 135-162 
Jaspers, F. (2007) Case Study Research: Some other applications besides theory building. Journal of Purchasing and Supply M anagement, Vol. 13 (3): 210-212

Jenkins, A., Babuk, T., and Mohitpour, M. (2005) Lowering Pipeline Costs Through Applied Technology. AACE International Transactions: 13.1-13.6 (AN 21750184)

Jenks, L. (1987) Back to Basics, or Whatever Happened to "the Customer is Always Right"? Journal of Health Care Marketing, Vol. 7(2): 2-5

Jermias, J. and Gani, L. (2004) Integrating business strategy, organizational configurations and management accounting systems with business unit effectiveness: a fitness landscape approach. Management Accounting Research, Vol. 15: 179-200

Johansson, T. and Siverbo, S. (2009) Why is Research on Management Accounting Change Not Explicitly Evolutionary? Taking the Next Step in the Conceptualisation of Management Accounting Change. M anagement Accounting Research, Vol. 20: 146-162

Johnson, R.B. (1997) Examining the validity structure of qualitative research. Education, Vol. 1118 (2): $282-292$

Jones, M.V. and Coviello, N.E. (2005) Internationalisation: Conceptualising an Entrepreneurial Process of Behaviour in Time. Journal of International Business Studies, Vol. 36 (3): 284-303.

Jones, M.J. and M ellett, H.J. (2007) Determinants of changes in accounting practices: Accounting and the UK Health Service. Critical Perspectives on Accounting, Vol. 18: 91-121

Joshi, P.L., Al-M udhaki, J., and Bremser, W.G. (2003) Corporate budget planning, control and performance evaluation in Bahrain. Managerial Auditing Journal, Vol. 18 (9): 737-750

Järvenpää, M . (2007) Making Business Partners: A Case Study of how Management Accounting Culture was Changed. European Accounting Review, Vol. 16 (1): $99-142$

Järvenpää, M. (2009) The institutional pillars of management accounting function. Journal of Accounting \& Organizational Change, Vol. 5 (4): 444-471

Järvinen, J. (2006) Institutional Pressures for Adopting New Cost Accounting Systems in Finnish Hospitals: Two Longitudinal Case Studies. Financial Accountability \& Management, Vol. 22 (1): 21-46

Kaplan, R.S. (1984) The Evolution of Management Accounting. The Accounting Review, Vol. 59, (3): $390-418$.

Karhunen, P. (2008) Managing international business operations in a changing institutional context: The case of St. Petersburg hotel industry. Journal of International M anagement, Vol. 14: 28-45

Karhunen, P., Löfgren, J., and Kosonen, R. (2008) Revisiting the relationship between ownership and control in international business operations: Lessons from transition economies. Journal of International M anagement, Vol. 14: 78-88

Kauffman, S.A. (2008) Reinventing the Sacred. Basic Books. Paperback published in 2010. United States of America. 320 pages 
Kerlinger, F.N. (1979) Foundations of Behavioral Research. Holt, Rinehart \& Winston, London and New York, United States of America. 741 pages

Khandwalla, P.N. (1973) Effect of Competition on the Structure of Top Management Control. Academy of Management Journal, Vol. 16 (2): 285-295

Kim, L. (1998) Crisis construction and organizational learning: Capability building in catching-up at Hyundai M otor. Organization Science, Vol. 9 (4): 506-521

Kirca, A.H. (2010) The effects of market orientation on subsidiary performance: Empirical evidence from MNCs in Turkey. Journal of World Business, In Press, Corrected Proof, Available online 29 October 2010.

Klein, P.G. (1998) New Institutional Economics. Available at SSRN: http://ssrn.com/abstract=115811 or doi:10.2139/ssrn.115811

Kholeif, A.O.R., Abdel-Kader, M., and Sherer, M. (2007) ERP customization failure: Institutionalized accounting practices, power relations and market forces. Journal of Accounting \& Organizational Change, Vol. 3 (3): 250-269.

Kohler, E.L. (1945) Expenditure Controls in the United States Government. The Accounting Review, Vol. 20 (1): 31-44

Knights, D. and McCabe, D. (1997) 'How Would You M easure Something Like That?': Quality in a Retail Bank. Journal of Management Studies, Vol. 34 (3): 371-388

Knutson, D.L. and Wichmann, H. (1985) The Accounting Standards Overload Problem for American Small Businesses. Journal of Business Finance \& Accounting, Vol. 12 (3): 387-397

Kober, R., Ng, J., and Paul, B.J. (2007) The interrelationship between management control mechanisms and strategy. Management Accounting Research, Vol. 18 (4): 425-452

Koch, J.V. and Cebula, R.J. (1994) In Search of Excellent Management. Journal of Management Studies, Vol. 31 (5): 681-699

Kotter, J.P. and Heskett, J.L. (1992) Corporate Culture and Performance. The Free Press. New York, United States of America. 214 pages.

Kurunmäki, L. (1999) Professional vs financial capital in the field of health care - struggles for the redistribution of power and control. Accounting, Organizations and Society. Vol. 24: 95-124

Kurunmäki, L., Lapsley, I. and Melia, K. (2003) Accountingization v. legitimation: a comparative study of the use of accounting information in intensive care. Management Accounting Research, Vol. 14 (2): 112-139

Langfield-Smith, K. (2008) Strategic management accounting: how far have we come in 25 years? Accounting, Auditing \& Accountability Journal, Vol. 21 (2): 204-228

Langfield-Smith, K. and Smith, D. (2003) Management control systems and trust in outsourcing relationships. M anagement Accounting Research, Vol. 14 (3): 281-307

Langlois, R.N. (1989) What was Wrong with the Old Institutional Economics (and What is Still Wrong with the New). Review of Political Economy. Vol. 1 (3): 270-298 
Lawlor, B. and Donnelly, R. (2010) Using podcasts to support communication skills development: A case study for content format preferences among postgraduate research students. Computers \& Education. Vol. 54: 962-971

Lea, B-R. and Min, H. (2003) Selection of management accounting systems in Just-In-Time and Theory of Constraints-based manufacturing. International Journal of Production Research, Vol. 41 (13): 2879-2910

Legge, K. (1970) The Operation of the 'Regressive Spiral' in the Labour Market. Journal of Management Studies, Vol. 7 (1): 1-22

Lehtonen, T. (2007) DRG-based prospective pricing and case-mix accounting-Exploring the mechanisms of successful implementation. Management Accounting Research, Vol. 18 (3): 367395

Levy, Y. and Ellis, T.J. (2006) A systems approach to conduct an effective literature review in support of information systems research. Informing Science Journal, Vol. 9: 181-211

Lincoln, Y.S. and Guba, E.G. (1985) Naturalistic Inquiry. Sage Publications. Beverly Hills, United States of America. 416 pages.

Lindgren, M. and Packendorff, J. (2006) What's New in New Forms of Organizing? On the Construction of Gender in Project-Based Work. Journal of M anagement Studies, Vol. 43 (4): 841866

Lindholm, A. and Suomala, P. (2005) Present and Future of Life Cycle Costing: Reflections from Finnish Companies. The Finnish Journal of Business Economics. Issue 2: 282-292.

Llewellyn, S. (1998) Boundary work: Costing and caring in the social services. Accounting, Organizations and Society, Vol. 23 (1): 23-47

Lovaglia, M.J., Willer, R. and Troyer, L. (2003) Power, status and collective action: developing fundamental theories to address a substantive problem. Advances in Group Processes, Vol. 20 (4): 495-511

Lowe, A., Doolin, B. (1999) Casemix accounting systems: new spaces for action. Management Accounting Research, Vol. 10 (3): 181-201

Lukka, K. (2007) Management Accounting Change and Stability: Loosely coupled rules and routines in action. M anagement Accounting Research, Vol. 18: 76-101

Lukka, K. and Granlund, M. (1996) Cost accounting in Finland: current practice and trends of development. The European Accounting Review, Vol. 5 (1): 1-28.

Lukka, K. and Modell, S. (2009) Validation in Interpretive Management Accounting Research. Accounting, Organizations and Society. doi: 10.1016/j.aos.2009.10.004

Länsiluoto, A. and Järvenpää, M. (2010) Collective action in the implementation of a "greener" performance measurement system. Journal of Accounting \& Organizational Change, Vol. 6 (2): 200-227

Macintosh, N.B. and Scapens, R.W. (1990) structuration theory in Management Accounting. Accounting, Organizations and Society, Vol. 15 (5): 455-477 
Macintosh, N.B. and Scapens, R.W. (1991) Management Accounting and Control Systems: A structuration theory Analysis. Journal of M anagement Accounting Research, Vol. 3: 131-158.

Maguire, S. and Phillips, N. (2008) 'Citibankers' at Citigroup: A Study of the Loss of Institutional Trust after a Merger. Journal of M anagement Studies, Vol. 45 (2): 372-401

Major, M. and Hopper, T. (2005) Managers divided: Implementing ABC in a Portuguese telecommunications company. Management Accounting Research, Vol. 16 (2): 205-229

Malhotra, M.K. and Grover, V. (1998) An Assessment of Survey Research in POM: from Constructs to Theory. Journal of Operations M anagement, Vol. 16: 407-425

Malmi, T. and Brown, D.A. (2008) Management control systems as a package - Opportunities, challenges and research directions. Management Accounting Research, Vol. 19: 287-300

Marginson, D. and Ogden, S. (2005) Coping with ambiguity through the budget: the positive effects of budgetary targets on managers' budgeting behaviours. Accounting, Organizations and Society, Vol. 30 (5): 435-456

Margolis, E. and Laurence, S. (1999) Concepts: Core Readings. The M IT Press. Cambridge (M A), United States of America. 652 pages

Masquefa, B. (2008) Top management adoption of a locally driven performance measurement and evaluation system: A social network perspective. M anagement Accounting Research, Vol. 19 (2): $182-207$

Matthews, R.C.O. (1986) The Economics of Institutions and the Sources of Growth. The Economic Journal, Vol. 96 (384): 903-918

Maunders, K. (1997) The AUTA/BAA 1971-1996: Living in Interesting Times. British Accounting Review, Vol. 29 (Special Issue): 63-79

Maxwell, J.A. (1992) Understanding and Validity in Qualitative Research. Harvard Educational Review, Vol. 62 (3): 279-300

Mayer, K.J. and Salomon, R.M. (2006) Capabilities, Contractual Hazards, and Governance: Integrating Resource-Based and Transaction Cost Perspectives. Academy of Management Journal, Vol. 49 (5): 942-959

McDermott, C.M., Greis, N.P. and Fischer, W.A. (1997) The diminishing utility of the product/process matrix. A study of the US power tool industry. International Journal of Operations \& Production M anagement, Vol. 17 (1): 65-84

M cGrath, J.E. and Brinberg, D. (1983) External Validity and the Research Process: A Comment on the Calder/Lynch Dialogue. Journal of Consumer Research, Vol. 10 (1): 115-124

McPhee, R.D. (1990) Approaches to integrating Longitudinal Case Studies. Organization Science, Vol. 1 (4): 393-405.

Menon, N.M., Lee, B., and Eldenburg, L. (2000) Productivity of Information Systems in the Healthcare Industry. Information Systems Research, Vol. 11 (1): 83-92 
Menon, N.M., Yaylacicegi, U., and Cezar, A. (2009) Differential Effects of the Two Types of Information Systems: A Hospital-Based Study. Journal of Management Information Systems, Vol. 26 (1): $297-316$

Mia, L. and Clarke, B. (1999) M arket competition, management accounting systems and business unit performance. M anagement Accounting Research, Vol. 10: 137-158

Miller, D. (1987) The Genesis of Configuration. The Academy of Management Review, Vol. 12 (4): 686-701

Moore, D.R.J. (2011) Structuration theory: The contribution of Norman Macintosh and its application to emissions trading. Critical Perspectives on Accounting, Vol. 22: 212-227

Morris, J.A. and Feldman, D.C. (1996) The dimensions, antecedents, and consequences of emotional labor. Academy of M anagement Review, Vol. 21(4): 986-1010

Morse, J.M., Barrett, M., Mayan, M., Olson, K., and Spiers, J. (2002) Verification Strategies for Establishing Reliability and Validity in Qualitative Research. International Journal of Qualitative Methods, Vol. 1 (2): 13-22

Mouly, V.S. and Sankaran, J.K. (2004) Survival and Failure of Small Businesses Arising Through Government Privatization: Insights from Two New Zealand Firms. Journal of Management Studies, Vol. 41 (8): 1435-1467

Mouritsen, J., Hansen, A. and Hansen, C.O. (2009) Short and long translations: Management accounting calculations and innovation management. Accounting, Organizations and Society, Vol. 34 (6-7): 738-754

Mullins, L.J. (2008) Essentials of Organizational Behaviour. Pearson Education Limited. Second Edition. Essex, United Kingdom. 517 pages

Murphy, G.L. (2002) The Big Book of Concepts. M IT Press: Cambridge (MA), United States of America. 555 pages

Murray, A.I. and Reshef, Y. (1988) American Manufacturing Unions' Stasis: A Paradigmatic Perspective. The Academy of M anagement Review, Vol. 13 (4): 615-626

Nandan, R. (2010) Management Accounting Needs of SMEs and the Role of Professional Accountants: A Renewed Research Agenda. Journal of Applied Management Accounting Research, Vol. 8 (1): 65-77

Nandhakumar, J. and Jones, M. (2001) Accounting for time: managing time in project-based teamworking. Accounting, Organizations and Society, Vol. 26 (3): 193-214

Naranjo-Gil, D. (2009) Management information systems and strategic performances: The role of a top team composition. International Journal of Information M anagement, Vol. 29: 104-110

Narver, J.C., and Slater, S.F. (1990) The Effect of a Market Orientation on Business Profitability. Journal of Marketing, Vol. 54 (4): 20-35

Nelson, R.R. (1995) Recent Evolutionary Theorizing About Economic Change. Journal of Economic Literature, Vol. 33 (1): 48-90 
Nelson, R.R. and Winter, S.G. (1982) An Evolutionary Theory of Economic Change. Harvard University Press. Cambridge (M A), United States of America. 437 pages.

Newman, K.L. (2000) Organizational Transformation During Institutional Upheaval. Academy of Management Review, Vol. 25 (3): 602-619

Nokelainen, T. (2008) A Typology of Competitive Actions. Tampere University of Technology, Tampere. 242 pages

Nor-Aziah A.K., Scapens R.W. (2007) Corporatisation and accounting change: The role of accounting and accountants in a M alaysian public utility. M anagement Accounting Research, Vol. 18 (2): $209-247$

North, D.C. (1990) Institutions, Institutional Change and Economic Performance. Cambridge University Press. Cambridge, United Kingdom. 159 pages

Nutt, P.C. (1983) Implementation Approaches for Project Planning. Academy of Management Review, Vol. 8 (4): 600-611

Oak, S. and Schmidgall, R.S. (2009) Have budgetary controls in the US club industry changed from the mid-1980s to the twenty-first century? International Journal of Contemporary Hospitality Management, Vol. 21 (4): 411-421

Ogburn, W.F. (1947) On Scientific Writing. The American Journal of Sociology, Vol. 52 (5): 383388

Ogden, S.G. (1995a) Transforming frameworks of accountability: The case of water privatization. Accounting, Organizations and So ciety, Vol. 20 (2-3): 193-218

Ogden, S.G. (1995b) Profit sharing and organizational change: attempts to promote employee commitment in the newly privatized water industry in England and Wales. Accounting, Auditing $\&$ Accountability Journal, Vol.8 (4): 23-47

Oliver, C. (1992) The Antecedents of Deinstitutionalization. Organization Studies, Vol. 13 (4): 563588

Olson, M.H. and Ives, B. (1982) Chargeback Systems and User Involvement in Information Systems - An Empirical Investigation. M IS Quarterly, Vol. 6 (2): 47-60

Orton, J.D. and Weick, K.E. (1990) Loosely Coupled Systems: A Reconceptualization. Academy of Management Review, Vol. 15 (2): 203-223

Ouchi, W.G. and Wilkins, A.L. (1985) Organizational Culture. Annual Review of Sociology, Vol. 11: 457-483

Owen, A.S. (2003) Measuring large UK accounting firm profit margins, mergers and concentration: A political economy of the accounting firm. Accounting, Auditing \& Accountability Journal, Vol. 16 (2): 275-297

Oxford Dictionary of English (2006) Oxford University Press. Second Edition. Italy. 2088 pages

Paul, S. (1997) Implementing an outpatient congestive heart failure clinic: The nurse practitioner role. Heart \& Lung: The Journal of Acute and Critical Care, Vol. 26 (6): 486-491 
Perera, S., McKinnon, J.L. Harrison, G.L. (2003) Diffusion of transfer pricing innovation in the context of commercialization-a longitudinal case study of a government trading enterprise. Management Accounting Research, Vol. 14 (2): 140-164

Piercy, N.F., Harris, L.C., Lane, N. (2002) Market orientation and retail operatives' expectations. Journal of Business Research. Vol. 51 (2): 157-166

Pizzini, M.J. (2006) The relation between cost-system design, managers' evaluations of the relevance and usefulness of cost data, and financial performance: an empirical study of US hospitals. Accounting, Organizations and Society, Vol. 31 (2): 179-210

Potter, B.N. (2005) Accounting as a Social and Institutional Practice: Perspective to Enrich our Understanding of Accounting Change. Abacus, Vol. 41 (3): 265-289

Powell, W.W. and DiM aggio, P.J. (1991) The New Institutionalism in Organizational Analysis. The University of Chicago Press. United States of America. 478 pages

Provan, K.G. (1987) Environmental and Organizational Predictors of Adoption of Cost Containment Policies in Hospitals. Academy of Management J ournal, Vol. 30 (2): 219-239

Quinn, M. (2011) Routines in management accounting research: further exploration. Journal of Accounting \& Organizational Change, Vol. 7 (4): 337-357

Rainer, R.K. and Watson, H.J. (1995) The Keys to Executive Information System Success. Journal of M anagement Information Systems, Vol. 12 (2): 83-98

Rasid, S.Z.A. Rahman, A.R.A. and Ismail, W.K.W. (2011) Management Accounting and Risk Management in Malaysian Financial Institutions. Managerial Auditing Journal, Vol. 26 (7): 566585

Rautiainen, A. and Järvenpää, M. (2012) Institutional Logics and Responses to Performance M easurement Systems. Financial Accountability \& M anagement, Vol. 28 (2): 164-188

Ribeiro, J.A. and Scapens, R.W. (2006) Institutional theories in management accounting change. Contributions, issues and paths for development. Qualitative Research in Accounting \& Management, Vol. 3(2): 94-111

Rindova, V.P. Pollock, T.G., and Hayward, M.L.A. (2006) Celebrity Firms: The Social Construction of M arket Popularity. Academy of M anagement Review, Vol. 31 (1): 50-71

Robbins, S.P. and Judge, T.A. (2008) Essentials of Organizational Behaviour. Pearson Education, Inc., Ninth Edition. New Jersey, United States of America. 324 pages.

Roberts, J., and Scapens, R.W. (1985) Accounting systems and systems of accountability: Understanding accounting practices in their organisational contexts. Accounting, Organizations, and Society. Vol. 10: 443-456

Rom, A., and Rohde, C. (2007) Management accounting and integrated information systems: A literature review. International Journal of Accounting Information Systems. Vol. 8: 40-68

Rowley, J., and Slack, F. (2004) Conducting a Literature Review. Management Research News, Vol. 27 (6): 31-39 
Rutherford, M. (2001) Institutional Economics: Then and Now. The Journal of Economic Perspectives, Vol. 15 (3): 173-194.

Said, J., Ghani, E.J., and Ibrahim, Z. (2011) Assessment of Computerized M anagement Accounting System among Small and Medium Size Construction Companies. Issue 39: 32-39

Salehi, M., Rostami, V. and M ogadam, A. (2010) Usefulness of Accounting Information System in Emerging Economy: Empirical Evidence of Iran. International Journal of Economics and Finance, Vol. 2 (2): 186-195

Saravanamuthu, K. (2008) Reflecting on the Biggs-Watkins theory of the Chinese Learner. Critical Perspectives on Accounting, Vol. 19: 138-180

Scapens, R.W. (1994) Never M ind the Gap: Towards an Institutional Perspective on M anagement Accounting Change. Management Accounting Research, Vol. 5: 301-321

Scapens, R.W. (2006) Understanding M anagement Accounting Practices: A Personal Journey. The British Accounting Review, Vol. 38: 1-30.

Scapens, R.W. and Roberts, J. (1993) Accounting and Control: A Case Study of Resistance to Accounting Change. M anagement Accounting Research, Vol. 4: 1-32

Schein, E.H. (2004) Organizational Culture and Leadership. Jossey-Bass. Third Edition. United States of America. 437 pages

Scott, W.H. (1918) Consciousness and Self-Consciousness. The Philosophical Review, Vol. 27 (1): $1-20$

Scott, W.R. (1987) The Adolescence of institutional theory. Administrative Science Quarterly, Vol. 32 (4): 493-511

Seal, W. (2006) Management accounting and corporate governance: An institutional interpretation of the agency problem. Management Accounting Research, Vol. 17 (4): 389-408.

Seal, W., and Croft, L. (1997) Professional rivalry and changing management control approaches in UK clearing banks. Accounting, Auditing \& Accountability Journal, Vol. 10 (1): 60-84

Selznick, P. (1996) Institutionalism "Old" and "New". Administrative Science Quarterly, Vol. 41 (2): 270-277.

Sewell, W.H. (1992) A Theory of Structure: Duality, Agency, and Transformation, Vol. 98 (1): 1-29

Shields, M.D. (1997) Research in Management Accounting by North Americans in the 19901s. Journal of M anagement Accounting Research, Vol. 9: 3-61

Shields, M .D., and Young, S.M . (1993) Antecedents and consequences of participative budgeting: Evidence on the effects of asymmetrical information. Journal of Management Accounting Research, Vol. 5: 265-280

Shields, M., and Young, S.M. (1994) Managing Innovation Costs: A Study of Cost Conscious Behavior by R\&D Professionals. Journal of M anagement Accounting Research, Vol. 6: 175-196

Siggelkow, N. (2002) Evolution toward Fit. Administrative Science Quarterly, Vol. 47 (1): 125-159

Singer, F.A. (1961) M anagement Accounting. The Accounting Review, Vol.36 (1): 112-118. 
Siti-Nabiha, A.K. and Scapens, R.W. (2005) Stability and change: an institutionalist study of management accounting change. Accounting, Auditing \& Accountability Journal, Vol. 18 (1): 4473

Smith, A.R. (1967) Manpower Planning in Management of the Royal Navy. Journal of Management Studies, Vol. 4 (2): 127-139

Smith, M. (1980) An Analysis of Three Managerial Jobs Using Repertory Grids. The Journal of Management Studies, Vol. 17 (2): 205-213

Smith, H.L., Fottler, M.D., and Saxberg, B.O. (1981) Cost Containment in Health Care: A M odel for Management Research. Academy of Management Review, Vol. 6 (3): 397-407

Smith, M.A., Mitra, S.A., and Narasimhan, S. (1998) Information Systems Outsourcing: A Study of Pre-Event Firm Characteristics. Journal of M anagement Information Systems, Vol. 15 (2): 61-93

Snyder, H. and Davenport, E. (1997) What does it really cost? Allocating indirect costs. Asian Libraries, Vol. 16 (3/4): 205-214

Snyder, K.D., Paulson, P., and McGrath, P. (2005) Improving processes in a small health-care network. Business Process Management, Vol. 11 (1): 87-99

Soin, K., Seal, W., and Cullen, J. (2002) ABC and organizational change: an institutional perspective. Management Accounting Research, Vol. 13: 249-271

Spraakman, G. (2006) The impact of institutions on management accounting changes at the Hudson's Bay Company, 1670 to 2005. Journal of Accounting \& Organizational Change, Vol. 2 (2): 101-122

Stajkovic, A.D. and Luthans, F. (2001) Differential Effects of Incentive Motivators on Work Performance. Academy of M anagement Journal, Vol. 4 (3): 580-590

Stenbacka, C. (2001) Qualitative Research Requires Quality Concepts of its Own. Management Decision, Vol. 39 (7): 551-555

Stewart, D.M. (2003) Piecing Together Service Quality: A Framework for Robust Service. Production and Operations M anagement, Vol. 12 (2): 246-265

Stonebraker, P.W., and Liao, J. (2004) Environmental turbulence, strategic orientation. M odeling supply chain integration. International Journal of Operations \& Production Management, Vol. 24 (9/10): 1037-1054

Straub, D.W. (1989) Validating Instruments in M IS Research. MIS Quarterly, Vol. 13 (2): 147-169

Strauss, G. (1962) Tactics of Lateral Relationship: The Purchasing Agent. Administrative Science Quarterly, Vol. 7 (2): 161-186

Strunk, W., and White, E.B. (1979) The Elements of style. McMillan. 3rd edition. New York, United States of America. 85 pages

Sum, C-C., Kow, L.S-J., and Chen, C-S. (2004) A taxonomy of operations strategies of high performing small and medium enterprises in Singapore. International Journal of Operations \& Production Management, Vol. 24 (3/4): 321-345 
Taggart, H. F. and Castenholz, W.B. (1935) Accounting and Code Regulation. The Accounting Review, Vol. 10 (1): 69-76

Tallon, P.P. (2010) A Service Science Perspective on Strategic Choice, IT, and Performance in U.S. Banking. Journal of M anagement Information Systems, Vol. 26 (4): 219-252

Tam, K.Y. (1996) Dynamic Price Elasticity and the Diffusion of Mainframe Computing. Journal of M anagement Information Systems, Vol. 13 (2): 163-183

Tam, S., Lee, W.B., Chung, W.W.C., and Nam, E.L.Y. (2003) Design of a re-configurable workflow system for rapid product development. Business Process M anagement Journal, Vol. 9 (1): 33-45

Tan, K.C. and Wisner, J.D. (2003) A study of operations management constructs and their relationships. International Journal of Operations \& Production Management, Vol. 23 (11): 13001325

Tannenbaum, A.S. (1962) Control in Organizations: Individual Adjustment and Organizational Performance. Administrative Science Quarterly, Vol. 7 (2): 236-257

ter Bogt, H.J. (2003) Performance evaluation styles in governmental organizations: How do professional managers facilitate politicians' work? Management Accounting Research, Vol. 14 (4): 311-332

ter Bogt, H.J. (2008) Management Accounting Change and New Public Management in Local Government: A reassessment of Ambitions and Results - An institutionalist Approach to Accounting Change in the Dutch Public Sector. Financial Accountability and M anagement, Vol. 24 (3): 209-241

ter Bogt, H.J. and van Helden, G.J. (2011) The role of consultant-researchers in the design and implementation process of a programme budget in a local government organization. Management Accounting Research, Vol. 22: 56-64

Thompson, J.D. and Bates, F.L. (1957) Technology, Organization, and Administration. Administrative Science Quarterly, Vol. 2 (3): 325-343

Tillema, S. (2005) Towards an integrated contingency framework for MAS sophistication. Case studies on the scope of accounting instruments in Dutch power and gas companies. Management Accounting Research, Vol. 16: 101-129

Tractinsky, N. and Jarvenpaa, S.L. (1995) Information Systems Design Decisions in a Global versus Domestic Context. MIS Quarterly, Vol. 19 (4): 507-534

Trueger, P.M. (1967) Contractor's Weighted Average Share in Cost Risk (CWAS). Journal of Accountancy, Vol. 123 (5): 47-55

Tsui, J.S.K. (2001) The impact of culture on the relationship between budgetary participation, management accounting systems, and managerial performance: An analysis of Chinese and Western managers. The International Journal of Accounting, Vol. 36: 125-146

Tuan Mat, T.Z., Smith, M., and Djajadikerta, H. (2010) Management Accounting and Organisational Change: An Exploratory Study in Malaysian Manufacturing Firms. Journal of Applied Management Accounting Research, Vol. 8 (2): 51-80 
Tucker, B. (2010) Through Which Lens? Contingency and Institutional Approaches to Conceptualising Organisational Performance in the Not-for-Profit Sector. Journal of Applied Management Accounting Research, Vol. 8 (1): 17-33

Tucker, B., Thorne, H., and Gurd, B. (2009) Managemetn Control Systems and Strategy: What's Been Happening? Journal of Accounting Literature, Vol. 28: 123-163

Tyrrall, D. and Parker, D. (2005) The Fragmentation of a Railway: A Study of Organizational Change. Journal of M anagement Studies, Vol. 42 (3): 507-537

van der Steen, M. (2011) The Emergence and Change of Management Accounting Routines. Accounting, Auditing \& Accountability Journal, Vol. 24 (4): 502-547

van Helden, G.J. (1997) Cost allocation and product costing in Dutch local government. European Accounting Review, Vol. 6 (1): 131-145

van Raaij, E.M., and Stoelhorst, J.W. (2008) The implementation of a market orientation: A review and integration of the contributions to date. European Journal of Marketing, Vol. 42 (11/12): 1265-1293

Veblen, T. (1898) Why is Economics Not an Evolutionary Science? The Quarterly Journal of Economics, Vol.12 (4): 373-397.

Veblen, T. (1909) The Limitations of M arginal Utility. The Journal of Political Economy, Vol. 17 (9): 620-636.

Wahyudi, I. (2009) From physical to accounting control: a study of accounting change resistance. Journal of Accounting \& Organizational Change, Vol. 5 (2): 228-242

Walsh, J.P. (1995) Managerial and Organizational Cognition: Notes from a Trip Down the Memory Lane. Organization Science, Vol. 6 (3): 280-321

Wanderley, C.D.A., Miranda, L.C., Matos de Meira, J., and Cullen, J. (2011) Management Accounting Change: Theoretical Frameworks. Revista de Administração e Contabilidade da Unisinos, Vol. 8 (2): 111-121

Webster, J. and Watson, R.T. (2002) Analyzing the past to prepare for the future: Writing a literature review. MIS Quarterly, Vol. 26 (2): 13-23

Weiss, J.W., Thorogood, A., and Clark, K.D. (2006) Three IT-Business Alignment Profiles: Technical Resource, Business Enabler, and Strategic Weapon. Communications of the Association for Information Systems, Vol. 18: 676-691

West, J. and Dedrick, J. (2000) Innovation and Control in Standards Architecture: The Rise and Fall of Japan's PC-98. Information Systems Research, Vol. 11 (2): 197-216

Whipp, R., Rosenfeld, R., and Pettigrew, A. (1989) Culture and Competitiveness: Evidence from Two Mature UK Industries. Journal of Management Studies, Vol. 26 (6): 561-585

Wickramasinghe, G. and M ills, L. (2002) Integrating e-commerce and knowledge management what does the Kaiser experience really tell us. International Journal of Accounting Information Systems, Vol. 3: 83-98 
Wilkinson, M., Fogarty, M., and Melville, D. (1996) Organizational culture change through training and cultural immersion. Journal of Organizational Change M anagement Vol. 9 (4): 69-81

Williamson, O.E. (1998). Transaction Cost Economics: How it Works, Where it is Headed. De Economist, Vol. 143 (1): 23-58

Williamson, O.E. (2000) The Institutional Economics: Taking Stock, Looking Ahead. Journal of Economic Literature, Vol. 38 (3): 595-613

Wood, S. (1996) High Commitment M anagement and Payment Systems. Journal of M anagement Studies, Vol. 33 (1): 53-77

Woodside, A.G. (2005) Market-Driven Thinking: Achieving Contextual Intelligence. Chapter 2Case Study Research Methods for Learning How Executives and Customers Think, Decide, and Act. Elsevier Butterworth-Heinemann. United States of America: 2-17

Woodward, S.N. (1982) Performance in Planning a Large Project. Journal of Management Studies, Vol. 19 (2): 183-198

Wright, E.R., Gronfein, W.P., and Owens, T.J. (2000) Deinstitutionalization, Social Rejection, and the Self-Esteem of Former M ental Patients. Journal of Health and Social Behavior, Vol. 41 (1): 6890

Yin, R.K. (1994) Case Study Research. Design and Methods. Sage Publications. Second Edition. United States of America. 171 pages

Young, A. (1991) The M arket Crash: Changes and Perspectives. Journal of Accounting, Auditing \& Finance, Vol. 6 (1): 129-133

Young, L.F. (1981) The Golden Scope Syndrome, the Availability Effect, and MIS. MIS Quarterly, Vol. 5 (3): 29-33.

Zengin, Y. and Ada, E. (2010) Cost management through product design: target costing approach. International Journal of Production Research, Vol. 48 (19): 5593-5611

Zott, C. and Huy, Q.N. (2007) How Entrepreneurs Use Symbolic Management to Acquire Resources. Administrative Science Quarterly, Vol. 52 (1): 70-105

Zucker, L.G. (1987) Institutional Theories of Organization. Annual Review of Sociology, Vol. 13: 443-464 


\section{Appendices}

\section{Appendix A. Journals and number of articles (in parenthesis) mentioning cost consciousness in the Title, Abstract or Keyword identified from the EBSCOhost, Elsevier Science Direct, JSTOR, Emerald and ABI.}

AACE International Transactions (3); ABA Banking Journal (3); ABA Journal (2); Academy of Management/The Academy of Management Review (1); Accounting, Auditing \& Accountability Journal (1); Accounting, Organizations \& Society (1); ACM Journal on Emerging Technologies in Computing Systems (1); Acta Astronautica (1); Agricultural Economics Review (1); American Association of Cost Engineers (4); American Economic Review (1); American Journal of Audiology (1); American Journal of Infection Control (1); American Journal of Obstetrics and Gynecology (1); American Journal of Transplantation (1); American Libraries (1); American Psychologist (1); American Review of Public Administration (1); American Sociologist (1); Anesthesiology Clinics of North America (1); Annals of Emergency Medicine (3); Annals of Internal Medicine (2); Annals of Regional Science (1); ANZ Journal of Surgery (1); Archives of Internal M edicine (1); ASHRAE Journal (1); Baillière's Clinical Anaesthesiology (1); Baillière's Clinical Rheumatology (1); Baylor Business Review (1); Benchmarking: An International Journal (2); Benefits Quarterly (1); Best Practice \& Research Clinical Anaesthesiology (1); BMC Health Services Research (1); Bone Marrow Transplantation (1); British Journal of Haematology (2); British Journal of Nursing (2); British Journal of Plastic Surgery (1); British Journal of Surgery (1); British Medical Journal (2); Brookings Review (1); Bulletin of Economic Research (1); Business and Society (1); Business Economics (1); Business Forum (2); Business Horizons (3); Business Lawyer (1); Business Strategy Review (2); Business: Theory \& Practice (1); Canadian Public Policy / Analyse de Politiques (1); Challenge (1); Change (1); Chemical Engineering (1); Civil Engineering (3); Climate Policy (1); Clinical Biochemistry (1); Clinical Oncology (1); Clinical Radiology (1); Clinical Therapeutics (1); Cognitive and Behavioral Practice (1); Columbia Journal of World Business (1); Communications in Statistics: Theory \& M ethods (1); Compensation \& Benefits Review (1); Computer (1); Computer Methods \& Programs in Biomedicine (1); Computer Standards \& Interfaces (1); Computers \& Operations Research (1); Computers \& Security (1); Consulting Psychology Journal: Practice \& Research (1); Contract M anagement (1); Cost Engineering (4); Crime, Law and Social Change (1); Current Anaesthesia \& Critical Care (1); Decision Sciences Journal of Innovative Education (1); Design News (2); Economic and Political Weekly (2); Education (1); Education + Training (1); Electrical Construction and Maintenance (1); Employee Relations (1); Endocrinology \& Metabolism Clinics of North America (1); Energy Policy (1); Engineering Construction $\&$ Architectural Management (1); Environmental Science \& Technology (1); European Accounting Review (1); European Journal of Operational Research (1); European Management Journal (1); European Psychiatry (1); Evaluation $\&$ the Health Professions (1); Facilities (1); Family Practice Management (1); Far Eastern Economic Review (2); Fertility and Sterility (1); Financial Accountability \& Management (1); Fire Engineering (1); Food Technology (1); Formulary (2); Futures (1); Gastrointestinal Endoscopy (1); Government Accountants J ournal (1); Harvard Health Letter (1); Health \& Social Work (1); Health Affairs (11); Health Care Management Review (2); Health Economics in Prevention and Care (1); Health Manpower Management (1); Health Marketing Quarterly (2); Health Policy (7); Health Services Research (1); Heart \& Lung: The Journal of Acute and Critical Care (2); Hematology/Oncology Clinics of North America (1); HFM - Healthcare Financial Management (1); Higher Education (1); Historical Journal of Film, Radio \& Television (1); 
Hospital \& Health Services Administration (2); Hospital Materiel Management Quarterly (1); Human Pathology (1); IEEE Computer Graphics \& Applications (1); IEEE Software $\quad$ (1); IEEE Spectrum (1); IEEE Transactions on Computers (1); Industrial \& Commercial Training (2); Industrial Management (1); Industrial Management \& Data Systems (1); Industrial Relations (1); Information Economics and Policy (1); Information Knowledge Systems M anagement (1); Information Management (1); Information Systems (1); Injury (1); Inquiry - Blue Cross and Blue Shield Association (1); Insight - the Journal of the American Society of Ophthalmic Registered Nurses (1); Intellectual Property \& Technology Law Journal (1); Intensive and Critical Care Nursing (1); International Executive (1); International Journal of Cardiology (1); International Journal of Health Care Quality Assurance (4); International Journal of Market Research (1); International J ournal of M edical M arketing (1); International Journal of Nursing Studies (1); International Journal of Organizational Innovation (1); International Journal of Pediatric Otorhinolaryngology (2); International Journal of Physical Distribution \& Logistics $M$ anagement (1); International Journal of Production Economics (2); International Journal of Radiation Oncology*Biology*Physics (1); International Journal of Technology Management (1); International Marketing Review (1); Issues in Science \& Technology (1); JACC: Cardiovascular Imaging (1); JAM A: Journal of the American M edical Association (4); Journal of Accountancy (5); Journal of Accounting, Auditing \& Finance (1); Journal of Advanced Nursing(2); Journal of Advertising (2); Journal of Advertising Research (1); Journal of Business (1); Journal of Business Administration (1); Journal of Chemical Education (1); Journal of Clinical Anesthesia (2); Journal of Clinical Nursing (1); Journal of Clinical Pharmacy \& Therapeutics (1); Journal of Consumer Marketing (1); Journal of Contemporary Business (1); Journal of E-Governance (1); Journal of Electrocardiology (1); Journal of European Industrial Training (1); Journal of Fashion M arketing \& M anagement

(2); Journal of Financial Service Professionals (1); Journal of Health \& Human Services Administration (1); Journal of Health Care Finance (1); Journal of Health Care Marketing (3); Journal of Health Economics (1); Journal of Health Organization and M anagement (1); Journal of Health Politics, Policy and Law (1); Journal of Hospital Infection (1); Journal of International Marketing \& Marketing Research (1); Journal of Management Accounting Research (1); Journal of Management Development (1); Journal of Managerial Psychology (1); Journal of Medical Marketing (1); Journal of Mental Health Administration (1); Journal of Mental Health Counseling (1); Journal of Microscopy (1); Journal of Modelling in Management (1); Journal of Occupational \& Environmental Medicine (1); Journal of PeriAnesthesia Nursing (1); Journal of Perinatology (1); Journal of Petroleum Technology (2); Journal of Private Equity (1); Journal of Professional Nursing (1); Journal of Property Management (1); Journal of Public Policy \& Marketing (1); Journal of Purchasing and M aterials M anagement (1); J ournal of Risk \& Insurance (5); Journal of Shoulder and Elbow Surgery (1); Journal of Small Business Management (1); Journal of Sound and Vibration (1); Journal of Systems Management (1); Journal of the Academy of Marketing Science (1); Journal of the American College of Cardiology (1); Journal of the American College of Radiology (1); Journal of the American M edical Directors Association (1); Journal of the American Psychiatric Nurses Association (1); Labor Law Journal (4); Library \& Information Science Research (1); Management Accountant (2); Management Accounting (2); Management Accounting Research (1); Management Decision (1); Management of Personnel Quarterly (1); Management Review (8); Management Services (6); Managing Leisure (1); Managing Service Quality (1); Marine Technology and SNAME News (1); Marketing Intelligence \& Planning (1); M aterials Performance (1); M edical Care (6); M edical Clinics of North America (1); Medical Teacher (1); Midwest Review of Public Administration (1); Multinational Monitor (1); Nature Biotechnology (1); Nature Reviews Drug Discovery (1); 
New England Journal of Entrepreneurship (1); New England Journal of Medicine (3); New Library World (4); Nursing (1); Nursing Economics (4); Nursing M anagement (3); Nutrition Today (1); Online Information Review (1); Operative Techniques in Orthopaedics (1); Organisation for Economic Cooperation and Development. The OECD Observer (1); Otolaryngology - Head and Neck Surgery (1); Patient Education and Counseling (1); Pattern Recognition Letters (2); Pediatrics (1); Pharmaceuticals Policy \& Law (1); PharmacoEconomics (1); Physical Therapy (1); Physics Today (1); Power (3); Practice Nurse (1); Primary Care Update for OB/GYNS (1); Production \& Inventory Management Journal (1); Professional Psychology: Research and Practice (1); Psychological Services (1); Public Administration Review (2); Public Budgeting \& Finance (1); Public Choice (1); Public Health Reports (1); Public Interest (1); Public M oney \& M anagement (2); Public Productivity Review (1); Public Relations Quarterly (1); Review of Economic Conditions in Italy (1); Review of Quantitative Finance \& Accounting (1); Reviews of Infectious Diseases (2); Risk Management (3); Schizophrenia Research (1); Science, New Series (1); Serials Review (1); Social Science \& Medicine (4); Social Work (1); Social Work in Health Care (2); Socio-Economic Planning Sciences (1); Sociology of Health \& IIIness (1); Southern Economic Journal (1); Stanford Law Review (1); Strategic Management Journal (2); Strategy \& Leadership (1); Surgical Clinics of North America (2); Survey of Ophthalmology (1); Systems \& Health: The Journal of Collaborative Family HealthCare (1); The American Journal of Cardiology (1); The American Journal of Medicine (4); The American Journal of Surgery (1); The Annals of Thoracic Surgery (1); The Bureaucrat (1); The Business Lawyer (1); The CPA Journal (2); The Economic Journal (1); The Health Care Manager (2); The Internal Auditor (1); The International Journal of Quality \& Reliability Management (1); The Journal of Business \& Industrial M arketing (1); The Journal of Business Forecasting Methods \& Systems (1); The Journal of Management Development (1); The Journal of the Institute for Socioeconomic Studies (1); The Journal of Urology (3); The Lancet (3); The Milbank M emorial Fund Quarterly (1); The Public Relations Journal (2); The Review of Economics and Statistics (1); The San Diego Law Review (1); The Social Service Review (1); The Yale Law Journal (1); Tooling \& Production (1); Tourism Management (1); Transfusion Science (1); Transport Reviews (1); Transportation Research Part A: Policy and Practice (1); UCLA Law Review (1); Urologic Clinics of North America (1); Urology (1); Water Environment \& Technology (1); Wear (1); Veterinary Parasitology (1); Virginia Law Review (1); World Pumps (1). 


\section{Appendix B. List of reviewed Journals and number of scrutinized articles in each}

Abacus (2); Academy of Management Journal (9); Academy of Management Review (5); Accounting and Business Research (1); Accounting and Finance; Accounting Horizons (4); Accounting, Auditing and Accountability Journal (6); Accounting, Management and Information Technologies (2000)/Information and Organization (2001); Accounting, Organizations and Society (19); Administrative Science Quarterly (10); Advances in Accounting Information Systems (1999)/International Journal of Accounting Information Systems (2000); Artificial Intelligence in Accounting and Auditing; Business Process M anagement Journal (2); Communications of the AIS (1); Contemporary Accounting Research (1); Critical Perspectives on Accounting (5); European Accounting Review (7); Financial Accountability and Management (4); Information Systems Journal (1); Information Systems Research (2); International Journal of Accounting Information Systems (2); International Journal of Operations and Production Management (6); Journal of Accounting and Economics; Journal of Accounting Research; Journal of Accounting, Auditing and Finance (2); Journal of Business Finance and Accounting (2); Journal of Information Systems; Journal of Management Accounting Research (3); Journal of Management Information Systems (8); Journal of Management Studies (15); Journal of the AIS; Logistics Information Management (2003)/Journal of Enterprise Information Management (2004) (1); M anagement Accounting Research (19); MIS Quarterly (6); Production and Operations Management (1); Review of Accounting Studies; The Accounting Review (16); The British Accounting Review (3); The Journal of Bank Cost and Management Accounting (2003)/Journal of Performance Management (2004) (1). 
Appendix C. List of Management Accounting Practices used in the questionnaire supporting the work of Chenhall and Langfield-Smith (1998)

Long Term Planning

- Formal strategic planning

- Capital budgeting techniques (e.g. NPV; IRR, Payback)

- Strategic plans developed:

- Together with budgets

- Separate from budgets

- Long range forecasting

Detailed budgeting systems for:

- Controlling costs

- Compensating managers

- Coordinating activities across the business units

- Linking financial position, resources and activities (e.g. activity based budgets)

- Planning:

- Day to day operations

- Cash flows

- Financial position

Product costing

- Absorption costing

- Variable costing

- Activity based costing

- Target costing

Performance evaluation based on:

- Budget variance analysis

- Controllable profit

- Divisional Profit

- Residual income (e.g. interest adjusted profit)

- Return (profit) on investment

- Cash flow return on investment

- Non-financial measures

- Team performance

- Employee attitudes

- Qualitative measures

- Balanced scorecard (mix of financial and non-financial measures)

- Customer satisfaction surveys

- Ongoing supplier evaluations

Decision support systems

- Cost-volume-profit analysis (e.g. breakeven analysis)

- Product life-cycle analysis 
- Activity based management

- Product profitability analysis

- Benchmarking of:

- product characteristics

- operational processes

- management processes

- strategic priorities

- Benchmarking carried out:

o within the wider organization

- with outside organizations

- Shareholder value analysis

- Value chain analysis

- Operations research techniques 


\section{Appendix D. Propositions and derivations by Lovaglia (2003)}

Proposition 1. The greater an actor's structural power, the greater the resources attained from exchange by that actor.

Proposition 2. The greater the resources attained through power use, the more negative the reaction to power use, including perceptions of selfishness and greed.

Proposition 3. The greater the expectations for an actor's contributions to the group, the greater that actor's status.

Proposition 4. The greater the influence of an actor over others in a group, the greater the expectations for that actor's contribution to the group.

Proposition 5. The greater an actor's resources, the greater will be the expectations for that actor's contributions to the group.

Proposition 6. The greater the negative reactions to power use, including perceptions of selfishness and greed, the lower the influence of the powerful.

Proposition 7. Effects of resources and expected self-motivation combine to determine an actor's influence.

Proposition 8. The more directly a person is subjected to power use, the more selfmotivated that person will perceive the power user to be.

Proposition 9. Self-presentation as group-motivated can reduce perceptions that a power user is selfishly motivated.

Proposition 10a. Philanthropy, voluntary contributions to a common good, reduces expectations of self-motivation and increases expectations for contribution to group goals.

Proposition 10b. Contribution to a public good increases expectations for the contributor's competence and perceptions of group motivation in a group that benefits from the public good.

Proposition 11. Status attainment has value to individuals

Derivation 1. Power users can increase their status by influencing observers of power use.

Derivation 2. Power users can increase their status through self-presentation as group motivated.

Derivation 3. Power users can increase their status through voluntary contributions to a common good (philanthropy).

Derivation 4. Contribution to public goods increases the status of contributors. 
Tampereen teknillinen yliopisto

PL 527

33101 Tampere

Tampere University of Technology

P.O.B. 527

FI-33101 Tampere, Finland

ISBN 978-952-15-2939-9

ISSN 1459-2045 